University of South Florida

DIGITAL COMMONS

Digital Commons @ University of

@ UNIVERSITY OF SOUTH FLORIDA

South Florida

USF Tampa Graduate Theses and Dissertations

USF Graduate Theses and Dissertations

9-16-2003

\title{
Graph-Theoretic Techniques for Web Content Mining
}

Adam Schenker

University of South Florida

Follow this and additional works at: https://digitalcommons.usf.edu/etd

Part of the American Studies Commons

\section{Scholar Commons Citation}

Schenker, Adam, "Graph-Theoretic Techniques for Web Content Mining" (2003). USF Tampa Graduate Theses and Dissertations.

https://digitalcommons.usf.edu/etd/1467

This Dissertation is brought to you for free and open access by the USF Graduate Theses and Dissertations at Digital Commons @ University of South Florida. It has been accepted for inclusion in USF Tampa Graduate Theses and Dissertations by an authorized administrator of Digital Commons @ University of South Florida. For more information, please contact digitalcommons@usf.edu. 
Graph-Theoretic Techniques for Web Content Mining

by

\begin{abstract}
Adam Schenker
A dissertation submitted in partial fulfillment

of the requirements for the degree of

Doctor of Philosophy

Department of Computer Science and Engineering

College of Engineering

University of South Florida
\end{abstract}

Major Professor: Abraham Kandel, Ph.D.

Dewey Rundus, Ph.D.

Horst Bunke, Ph.D.

Ken Christensen, Ph.D.

Carlos Smith, Ph.D.

Date of Approval:

September 16, 2003

Keywords: graph similarity, graph distance, machine learning, clustering, classification

(C) Copyright 2003 , Adam Schenker 


\section{Dedication}

For my parents. 


\section{Acknowledgements}

There are many people who helped contribute to the successful completion of this dissertation. First I would like to thank Dr. Abraham Kandel for supporting me with not only his scientific and monetary resources, but with his wisdom and kindness. Dr. Mark Last provided invaluable assistance in performing my research; his feedback always served to improve the work in meaningful ways. Dr. Horst Bunke was a tremendous help and source of knowledge; without his constant support and vision this dissertation would not have been possible. Dr. Dewey Rundus, Dr. Ken Christensen, Dr. Carlos Smith and Dr. José Zayas-Castro all provided useful comments and advice for improving the dissertation and their help is greatly appreciated. Finally, it is necessary for me to mention the staff and my fellow graduate students at the Department of Computer Science and Engineering each of whom has enabled me reach my goal: Ms. Judy Hyde, Dr. Scott Dick, Dr. Lawrence Hall, Dr. Rafael Perez, Ms. Sarah Burton and Mr. Daniel Prieto. 


\section{Table of Contents}

List of Tables $\quad$ iv

List of Figures vi

Abstract $\quad x$

$\begin{array}{ll}\text { Chapter One: Introduction } & 1\end{array}$

Chapter Two: A Review of Web Mining Techniques 6

2.1 Overview of Web Mining Methodologies 6

2.2 Traditional Information Retrieval Techniques for Plain-Text Documents 7

2.3 Web Search Clustering 9

2.4 Summary 11

Chapter Three: A Review of Graph Similarity Techniques 12

3.1 Introduction 12

3.2 Graph and Subgraph Isomorphism 13

3.3 Graph Edit Distance $\quad 15$

3.4 Maximum Common Subgraph / Minimum Common Supergraph Approach 16

3.5 State Space Search Approach 18

$\begin{array}{ll}3.6 \text { Probabilistic Approach } & 18\end{array}$

3.7 Distance Preservation Approach 20

3.8 Relaxation Approaches 21

3.9 Mean and Median of Graphs 23

3.10 Remarks 24

Chapter Four: Graph Models for Web Documents 26

4.1 Pre-Processing 26

4.2 Graph Representations of Web Documents 27

4.3 Complexity Analysis 31

Chapter Five: The Graph Hierarchy Construction Algorithm for Organizing

Web Search Results 32

5.1 Introduction $\quad 32$

5.2 Cluster Hierarchy Construction Algorithm (CHCA) 33

5.2.1 A Review of Inheritance 33 
5.2.2 Brief Overview of CHCA 34

5.2.3 CHCA in Detail 35

5.2.4 CHCA: an Example 39

5.2.5 Examination of CHCA as a Clustering Method 40

5.3 Application of CHCA to Search Results Processing 43

5.3.1 Asynchronous Search 43

5.3.2 Implementation, Input Preparation and Pre-processing 44

5.3.3 Selection of Parameters for Web Search 44

5.4 Examples of Results 46

5.4.1 Comparison with Grouper $\quad 46$

5.4.2 Comparison with Vivísimo $\quad 50$

5.5 Graph Hierarchy Construction Algorithm (GHCA) 52

5.5.1 Parameters 53

5.5.2 Graph Creation and Pre-processing 54

5.5.3 Graph Hierarchy Construction Algorithm (GHCA) 55

5.5.4 GHCA Examples $\quad 57$

5.6 Comments 58

Chapter Six: A Graph-Theoretical Extension of the $k$-Means Clustering Algorithm 61

6.1 Introduction 61

6.2 The Extended $k$-Means Clustering Algorithm 62

6.3 Web Document Data Sets 63

6.4 Clustering Performance Measures 64

6.5 Comparison with Published Results 65

6.6 Remarks 68

Chapter Seven: Comparison of Different Graph-Theoretical Distance Measures and Graph Representations for Graph-Theoretic Clustering 70

$\begin{array}{ll}7.1 \text { Introduction } & 70\end{array}$

7.2 Comparison of Distance Measures 73

7.3 Comparison of Graph Representations $\quad 77$

$\begin{array}{ll}7.4 \text { Visualization of Graph Clustering } & 79\end{array}$

Chapter Eight: The Graph-Theoretic Global k-Means Algorithm 82

8.1 Introduction $\quad 82$

8.2 Global $k$-Means vs. Random Initialization 83

8.3 Optimum Number of Clusters 85

Chapter Nine: A Graph-Theoretical Extension of the k-Nearest Neighbors

$\begin{array}{ll}\text { Classification Algorithm } & 89\end{array}$

$\begin{array}{ll}9.1 \text { Introduction } & 89\end{array}$

$9.2 k$-Nearest Neighbors with Graphs $\quad 89$

9.3 Experimental Results 90

9.4 Remarks 98 
$\begin{array}{ll}\text { References } & 103\end{array}$

Appendices $\quad 113$

Appendix A: Examples of Documents Used in Experiments 114

Appendix B: Graphs Created from Example Documents of Appendix A 120 Appendix C: Nearest Neighbors of Example Documents 123 $\begin{array}{ll}\text { Appendix D: Graphs of Nearest Neighbors } & 129\end{array}$

About the Author $\quad$ End Page 


\section{List of Tables}

Table 5.1 Simple Example to Illustrate Concepts of CHCA

Table 5.2 Results of the Grouper Custom System for the Query "Soft Computing"

Table 5.3 Results of the Grouper Custom System for the Query "Data Compression"

Table 5.4 List of Query Strings Used for Comparison

Table 5.5 Summary of Comparison for 10 Searches (C: CHCA, V: Vivísimo)

Table 6.1 Results of Our Experiments Compared with Results from Strehl et al.

Table 7.1 Clustering Performance Comparison for K-Series

Table 7.2 Distance Measure Comparison for K-Series

Table 7.3 Graph Representation Comparison for K-Series

Table 8.1 Results for F-Series (Rand Index) 84

Table 8.2 Results for F-Series (Mutual Information) $\quad 84$

Table 8.3 Results for J-Series (Rand Index) 84

Table 8.4 Results for J-Series (Mutual Information) 85

Table 8.5 Execution Times Using Random Initialization (in Seconds) 85

Table 8.6 Execution Times Using Global $k$-Means (in Minutes) 85

Table $8.7 \quad$ Results for F-Series Using Global $k$-Means $\quad 87$

Table $8.8 \quad$ Results for F-Series Using Random Initializations $\quad 88$ 
Table 8.9 Results for J-Series Using Global $k$-Means

Table 8.10 Results for J-Series Using Random Initializations 88

Table 9.1 Average Times to Classify One K-Series Document for Each Method 


\section{List of Figures}

Figure 3.1 A Graph G and its Compliment $\bar{G}$ May be Isomorphic 14

Figure 4.1 Example of a Standard Graph Representation of a Document 29

Figure 4.2 Example of a Simple Graph Representation of a Document 29

Figure 4.3 Example of an $n$-Distance Graph Representation of a Document 29

Figure 5.1 Summary of Notation Used in CHCA 34

Figure 5.2 Cluster Hierarchy Created from the Example in Table 5.1 40

Figure 5.3 Average Number of Clusters Created as a Function of Maximum Cluster Threshold (MCT) for Three Queries

Figure 5.4 Average Number of Clusters Created as a Function of Minimum Pages Threshold (MPT) for Three Queries 45

Figure 5.5 Cluster Hierarchy Created by CHCA for the Query "Soft Computing"

Figure 5.6 Cluster Hierarchy Created by CHCA for the Query "Data compression"

Figure 5.7 Pre-processing Phase of GHCA 53

Figure 5.8 Initial Hierarchy Construction Phase of GHCA 55

Figure 5.9 Document Assignment Phase of GHCA 55

Figure 5.10 Cluster Pruning Phase of GHCA 56

Figure 5.11 Results Display Methodology for GHCA 56

Figure 5.12 Examples of Cluster Hierarchies Generated by GHCA 58

$\begin{array}{lll}\text { Figure 6.1 The Traditional } k \text {-Means Clustering Algorithm } & 62\end{array}$ 
Figure 6.2 The Graph-Theoretic k-Means Clustering Algorithm

Figure 6.3 Mutual Information as a Function of the Maximum Number of Vertices per Graph

Figure 6.4 Clustering Time as a Function of the Maximum Number of Vertices per Graph

Figure 7.1 Distance Measure Comparison for the F-Series Data Set (Rand Index)

Figure 7.2 Distance Measure Comparison for the F-Series Data Set (Mutual Information)

Figure 7.3 Distance Measure Comparison for the F-Series Data Set (Dunn Index)

Figure 7.4 Distance Measure Comparison for the J-Series Data Set (Rand Index)

Figure 7.5 Distance Measure Comparison for the J-Series Data Set (Mutual Information)

Figure 7.6 Distance Measure Comparison for the J-Series data set (Dunn index)

Figure 7.7 Graph Representation Comparison for the F-Series Data Set (Rand Index)

Figure 7.8 Graph Representation Comparison for the F-Series Data Set (Mutual Information)

Figure 7.9 Graph Representation Comparison for the F-Series Data Set (Dunn Index)

Figure 7.10 Graph Representation Comparison for the J-Series Data Set (Rand Index)

Figure 7.11 Graph Representation Comparison for the J-Series Data Set (Mutual Information)

Figure 7.12 Graph Representation Comparison for the J-Series Data Set (Dunn Index) 
Figure 7.13 F-Series Data Set Represented by Graphs Scaled to Two Dimensions Using 10 Nodes/Graph (Left) and 30 Nodes/Graph (Right)

Figure 7.14 J-Series Data Set Represented by Graphs Scaled to Two Dimensions Using 10 Nodes/Graph (Left) and 30 Nodes/Graph (Right)

Figure 7.15 K-Series Data Set Represented by Graphs Scaled to Two Dimensions Using 70 Nodes/Graph (Left) and 100 Nodes/Graph (Right)

Figure 9.1 The Basic $k$-Nearest Neighbors Algorithm

Figure 9.2 Classification Accuracy for the F-Series Data Set

Figure 9.3 Classification Accuracy for the J-Series Data Set

Figure 9.4 Classification Accuracy for the K-Series Data Set

Figure 9.5 Distance Measure Comparison for the F-Series

Figure 9.6 Distance Measure Comparison for the J-Series

Figure 9.7 Distance Measure Comparison for the K-Series

Figure 9.8 Graph Representation Comparison for the F-Series

Figure 9.9 Graph Representation Comparison for the J-Series

Figure 9.10 Graph Representation Comparison for the K-Series

Figure 9.11 Combining WGU/MMCSN Distance Measure and Simple Representation for the J-Series

Figure A.1 Original HTML of Document \#68 of the J-Series Data Set (Partial)

Figure A.2 Original HTML of Document \#104 of the J-Series Data Set

Figure A.3 Original HTML of Document \#183 of the J-Series Data Set (Partial)

Figure A.4 Document of Fig. A.1 as Rendered in a Web Browser (Partial) 
Figure A.5 Document of Fig. A.2 as Rendered in a Web Browser 118

Figure A.6 Document of Fig. A.3 as Rendered in a Web Browser (Partial) 119

Figure B.1 Standard Graph Representation Created from Web Document of Fig. A.1 Using 10 Nodes/Graph

Figure B.2 Standard Graph Representation Created from Web Document of Fig. A.2 Using 10 Nodes/Graph

Figure B.3 Standard Graph Representation Created from Web Document of Fig. A.3 Using 10 Nodes/Graph

Figure C.1 HTML Source of Nearest Neighbor of Document of Fig. A.1 (Partial)

Figure C.2 HTML Source of Nearest Neighbor of Document of Fig. A.2 (Partial)

Figure C.3 HTML Source of Nearest Neighbor of Document of Fig. A.3 (Partial)

Figure C.4 Document of Fig. C.1 as Rendered in a Web Browser (Partial)

Figure C.5 Document of Fig. C.2 as Rendered in a Web Browser (Partial)

Figure C.6 Document of Fig. C.3 as Rendered in a Web Browser (Partial)

Figure D.1 Standard Graph Representation of Document of Fig. C.1

Figure D.2 Standard Graph Representation of Document of Fig. C.2

Figure D.3 Standard Graph Representation of Document of Fig. C.3 


\title{
Graph-Theoretic Techniques for Web Content Mining
}

\section{Adam Schenker}

\begin{abstract}
In this dissertation we introduce several novel techniques for performing data mining on web documents which utilize graph representations of document content. Graphs are more robust than typical vector representations as they can model structural information that is usually lost when converting the original web document content to a vector representation. For example, we can capture information such as the location, order and proximity of term occurrence, which is discarded under the standard document vector representation models. Many machine learning methods rely on distance computations, centroid calculations, and other numerical techniques. Thus many of these methods have not been applied to data represented by graphs since no suitable graphtheoretical concepts were previously available.

We introduce the novel Graph Hierarchy Construction Algorithm (GHCA), which performs topic-oriented hierarchical clustering of web search results modeled using graphs. The system we created around this new algorithm and its prior version is compared with similar web search clustering systems to gauge its usefulness. An important advantage of this approach over conventional web search systems is that the results are better organized and more easily browsed by users.

Next we present extensions to classical machine learning algorithms, such as the $k$-means clustering algorithm and the $k$-Nearest Neighbors classification algorithm, which allows the use of graphs as fundamental data items instead of vectors. We perform experiments comparing the performance of the new graph-based methods to the traditional vector-based methods for three web document collections. Our experimental results show an improvement for the graph approaches over the vector approaches for both clustering and classification of web documents. An important advantage of the graph representations we propose is that they allow the computation of graph similarity in polynomial time; usually the determination of graph similarity with the techniques we use is an NP-Complete problem. In fact, there are some cases where the execution time of the graph-oriented approach was faster than the vector approaches.
\end{abstract}




\section{Chapter One}

\section{Introduction}

With the recent explosive growth of the amount of content on the Internet, it has become increasingly difficult for users to find and utilize information and for content providers to classify and catalog documents. Traditional web search engines often return hundreds or thousands of results for a search, which is time consuming for users to browse. On-line libraries, search engines, and other large document repositories (e.g. customer support databases, product specification databases, press release archives, news story archives, etc.) are growing so rapidly that it is difficult and costly to categorize every document manually. In order to deal with these problems, researchers look toward automated methods of working with web documents so that they can be more easily browsed, organized, and cataloged with minimal human intervention.

In contrast to the highly structured tabular data upon which most machine learning methods are expected to operate, web and text documents are semi-structured. Web documents have well-defined structures such as letters, words, sentences, paragraphs, sections, punctuation marks, HTML tags, and so forth. We know that words make up sentences, sentences make up paragraphs, and so on, but many of the rules governing the order in which the various elements are allowed to appear are vague or illdefined and can vary dramatically between documents. It is estimated that as much as $85 \%$ of all digital business information, most of it web-related, is stored in non-structured formats (i.e. non-tabular formats, such as those that are used in databases and spreadsheets) [100]. Developing improved methods of performing machine learning techniques on this vast amount of non-tabular, semi-structured web data is therefore highly desirable.

Clustering and classification have been useful and active areas of machine learning research that promise to help us cope with the problem of information overload on the Internet. With clustering the goal is to separate a given group of data items (the data set) into groups called clusters such that items in the same cluster are similar to each other and dissimilar to the items in other clusters. In clustering methods no labeled examples are provided in advance for training (this is called unsupervised learning). Under classification we attempt to assign a data item to a predefined category based on a model that is created from pre-classified training data (supervised learning). In more general terms, both clustering and classification come under the area of knowledge discovery in databases or data mining. Applying data mining techniques to web page content is referred to as web content mining which is a new sub-area of web mining, partially built upon the established field of information retrieval.

When representing text and web document content for clustering and classification, a vector model is typically used [107]. In this model, each possible term 
that can appear in a document becomes a feature dimension. The value assigned to each dimension of a document may indicate the number of times the corresponding term appears on it or it may be a weight that takes into account other frequency information, such as the number of documents upon which the terms appear. This model is simple and allows the use of traditional machine learning methods that deal with numerical feature vectors in a Euclidean feature space. However, it discards information such as the order in which the terms appear, where in the document the terms appear, how close the terms are to each other, and so forth. By keeping this kind of structural information we could possibly improve the performance of various machine learning algorithms. The problem is that traditional data mining methods are often restricted to working on purely numeric feature vectors due to the need to compute distances between data items or to calculate some representative of a cluster of items (i.e. a centroid or center of a cluster), both of which are easily accomplished in a Euclidean space. Thus either the original data needs to be converted to a vector of numeric values by discarding possibly useful structural information (which is what we are doing when using the vector model to represent documents) or we need to develop new, customized methodologies for the specific representation.

Graphs are important and effective mathematical constructs for modeling relationships and structural information. Graphs (and their more restrictive form, trees) are used in many different problems, including sorting, compression, traffic/flow analysis, resource allocation, etc. [22] In addition to problems where the graph itself is processed by some algorithm (e.g. sorting by the depth first method or finding the minimum spanning tree) it would be extremely desirable in many applications, including those related to machine learning, to model data as graphs since these graphs can retain more information than sets or vectors of simple atomic features. Thus much research has been performed in the area of graph similarity in order to exploit the additional information allowed by graph representations by introducing mathematical frameworks for dealing with graphs. Some application domains where graph similarity techniques have been applied include face [132] and fingerprint [129] recognition as well as anomaly detection in communication networks [34]. In the literature, the work comes under several different topic names including graph distance, (exact) graph matching, inexact graph matching, error tolerant graph matching, or error correcting graph matching. In exact graph matching we are attempting to determine if two graphs are identical. Inexact graph matching implies we are attempting not to find a perfect matching, but rather a "best" or "closest" matching. Error tolerant and error correcting are special cases of inexact matching where the imperfections (e.g. missing nodes) in one of the graphs, called the data graph, are assumed to be the result of some errors (e.g. from transmission or recognition). We attempt to match the data graph to the most similar model graph in our database. Graph distance is a numeric measure of dissimilarity between graphs, with larger distances implying more dissimilarity. By graph similarity, we mean we are interested in some measurement that tells us how similar graphs are regardless if there is an exact matching between them.

In this dissertation we will be presenting important contributions that help improve the clustering and classification of web documents. This is accomplished by representing their content with a more versatile graph model, which can retain additional 
information that is not captured when using a vector representation. The Graph Hierarchy Construction Algorithm (GHCA) is a novel hierarchical clustering algorithm we developed and implemented in a system that hierarchically clusters web search results by topic. Important features of this clustering method aside from the use of graphs to represent web documents include labeling of clusters by topic, hierarchical ordering from general (top level) to more specific (lower level) clusters, multiple inheritance (clusters with more than one parent), and several parameters that allow user adjustment of the results. In comparison with similar systems our system has been shown to perform well. We will also describe graph-theoretical extensions to existing, proven clustering and classification methods that for the first time allow them to deal with data represented by graphs rather than vectors. This approach has two main benefits. First, it allows us to keep the inherent structure of the original data by modeling web document content as a graph, rather than having to arrive at numeric feature vectors that contain only term frequency information. Second, we do not need to develop new algorithms or frameworks to deal with the graphs: we can simply apply straightforward extensions to go from classical data mining algorithms that use numerical vectors to those that can handle graphs. It is our contention that by using graphs to keep information that is usually lost we can improve clustering and classification performance over the usual vector model for the same algorithm. We will explore this contention through a series of experiments, using the well known $k$-means clustering and $k$-Nearest Neighbors classification algorithms. A surprising realization during our experiments is that, with careful selection of the graph representation model for an application, we can achieve polynomial time complexity for the graph similarity procedure. In the general case this is an NP-Complete problem. Note that these techniques are not limited to web documents or even text documents in general: they allow any data sets that are complex enough to require representation by graphs (e.g. software code, computer networks, maps, images, etc.) to now be clustered or classified using classical, popular methods without loss of the inherent structural information.

The remainder of this dissertation is as follows. We give a definition and overview of the three areas of web mining in Chapter 2. We also give a review of the most relevant work concerning content-based web mining and web search result clustering systems in particular.

A thorough literature review of graph similarity techniques as well as the mathematical definitions and notation needed for the dissertation is presented in Chapter 3. We will review the maximum common subgraph approach, which is what we use for graph similarity in our web mining algorithms. We will also give introductions to some other related methods including graph isomorphism, graph edit distance, and median of a set of graphs. The five graph-theoretical distance measures we use will also be presented here.

In Chapter 4 we will show how web documents can be modeled as graphs and give some implementation details relating to the creation of document models from their content. We compare several graph representation approaches in our experiments, which are introduced here with examples. The complexity analysis related to these representations is also given. 
In Chapter 5 we introduce the Graph Hierarchy Construction Algorithm (GHCA), which is based on our earlier work on the Cluster Hierarchy Construction Algorithm (CHCA) [110][111]. We will present this novel algorithm which can accomplish the task of hierarchically clustering web search results returned from conventional web search engines. With CHCA, web pages are represented as sets of terms; GHCA uses a more robust graph representation that allows for the preservation of term phrases (ordering information) which provides a more coherent display of results to the user. From these representations we can create a cluster hierarchy that is a model of the application domain (in this case, the search topic). Such a hierarchy is actually a form of knowledge representation, similar to frames and semantic networks [76][106]. By creating a knowledge representation from web documents using GHCA, we allow for search results to be more easily browsed by users. Search results are grouped by topic and sub-topic rather than as a single, large ranked list. We have created a working prototype system which uses these algorithms to create a cluster hierarchy from actual search results that are returned from conventional search engines using a user's provided query string. GHCA creates appropriate clusters from the results and assigns pages to each cluster. This clustering of the results, along with the fact that the hierarchy structure provides information about the relationships between the clusters, helps the user by better organizing the results and providing additional information such as what the related topics are. We present a comparison to some other similar search result clustering systems in this chapter.

In extending vector-based CHCA to graph-oriented GHCA we have realized that many classical machine learning algorithms which typically use vectors can also be extended to deal with more robust graph representations in a similar way. Thus the second area we will investigate, which comprises the remainder of the dissertation, describes methods of utilizing graphs to represent web documents during clustering and classification using existing, well-known machine learning algorithms.

In Chapter 6 we will describe an extension to the $k$-means clustering algorithm that allows the utilization of graphs instead of vectors [112] and illustrate its usefulness by applying it to the problem of clustering a collection of web documents. We will define the clustering performance indexes that will be used to measure clustering performance as well as provide a description of the various data sets that are used in our experiments. Initial experimental results will be given and compared with previously published results reported for the same web data set based on a traditional vector representation and the usual $k$-means algorithm with various vector-based distance measures.

Our next series of experiments is presented in Chapter 7, where we address the comparison of different graph similarity measures and graph representations of web documents in the context of document clustering performance [113][115]. We will use the graph-oriented $k$-means clustering algorithm of Chapter 6 to cluster two web document collections when using one of five graph-theoretic distance measures. We will also cluster the web documents when represented by graphs using one of the six graph representation methodologies we have proposed. The clustering performance under each distance measure and graph representation will be measured by three clustering performance measures. Clustering performance as a function of graph size is also 
explored. Euclidean distance and cosine similarity measures are used with the traditional vector model representation of documents as a baseline for comparison.

In Chapter 8 we will measure the performance of our clustering method when combined with the global $k$-means algorithm presented in [71], which provides a deterministic method of finding "good" initial cluster center positions [117]. Previous experimental results have shown that initialization with global $k$-means can lead to clustering performance which is as good or better than random initializations, and we will investigate whether this holds true for our methods and data sets as well. We also use this method to examine the question of the optimum number of clusters for the document collections. We will use the global $k$-means initializations for a range of different $k$ values (numbers of clusters) and measure performance with additional cluster validity indexes.

In Chapter 9 we compare the traditional vector model representation to our new graph model in the context of the document classification task rather than clustering. We introduce a graph-based extension of the popular $k$-Nearest Neighbors classification algorithm [114][116] and measure classification accuracy using the leave-one-out approach over all three web document collections. We select several values for the number of nearest neighbors, $k$, and look at the classification performance as a function of the size of the graphs representing each document. We also examine the effect of different graph-theoretical distance measures and graph representations on classification accuracy as we did in Chapter 7. Further, we compare execution times of both our graphbased approach and the traditional vector approach using cosine similarity or Jaccard similarity.

Concluding remarks and possible future extensions of the current work will be given in Chapter 10. 


\section{Chapter Two}

\section{A Review of Web Mining Techniques}

\subsection{Overview of Web Mining Methodologies}

Web mining [140] is the application of machine learning (data mining) techniques to web-based data for the purpose of learning or extracting knowledge. Web mining encompasses a wide variety techniques, including soft computing [99]. Web mining methodologies can generally be classified into one of three distinct categories: web usage mining, web structure mining, and web content mining [78]. For a survey of techniques used in these areas, see [63]. In web usage mining the goal is to examine web page usage patterns in order to learn about a web system's users or the relationships between the documents. For example, the tool presented by Masseglia et al. [81] creates association rules from web access logs, which store the identity of pages accessed by users along with other information such as when the pages were accessed and by whom; these logs are the focus of the data mining effort, rather than the actual web pages themselves. Rules created by their method could include, for example, " $70 \%$ of the users that visited page $A$ also visited page $B$." Similarly, the method of Nasraoui et al. [95] also examines web access logs. The method employed in that paper is to perform a hierarchical clustering in order to determine the usage patterns of different groups of users. Beeferman and Berger [7] described a process they developed which determines topics related to a user query using click-through logs and agglomerative clustering of bipartite graphs. The transaction-based method developed in [87] creates links between pages that are frequently accessed together during the same session. Web usage mining is useful for providing personalized web services, an area of web mining research that has lately become active. It promises to help tailor web services, such as web search engines, to the preferences of each individual user. For a recent review of web personalization methods, see [37].

In the second category of web mining methodologies, web structure mining, we examine only the relationships between web documents by utilizing the information conveyed by each document's hyperlinks. Like the web usage mining methods described above, the other content of the web pages is often ignored. In [66] Kumar et al. examined utilizing a graph representation of web page structure. Here nodes in the graphs are web pages and edges indicate hyperlinks between pages. By examining these "web graphs" it is possible to find documents or areas of interest through the use of certain graphtheoretical measures or procedures. Structures such as web rings, portals, or affiliated sites can be identified by matching the characteristics of these structures (e.g. we can identify portal pages because they have an unusually high out-degree). Graph models are also used in other web structure mining approaches. For example, in [17] the authors' 
method examines linked URLs and performs classification using a Bayesian method. The graph is processed to determine groups of pages that relate to a common topic.

In this dissertation we are concerned only with the third category of web mining, web content mining. In web content mining we examine the actual content of web pages (most often the text contained in the pages) and then perform some knowledge discovery procedure to learn about the pages themselves and their relationships. Most typically this is done to organize a group of documents into related categories. This is especially beneficial for web search engines, since it allows users to more quickly find the information they are looking for in comparison to the usual "endless" ranked list. There are several examples of web or text mining approaches [3] that are content-oriented and attempt to cluster documents for browsing. The Athena system of Agrawal et al. [2] creates groupings of e-mail messages. The goal is to create folders (classes) for different topics and route e-mail messages automatically to the appropriate folders. Athena uses a clustering algorithm called C-Evolve to create topics (folders), while the classification of documents to each cluster is supervised and requires manual interaction with the user. The classification method is based on Naïve Bayes. Some notable papers that deal with clustering for web search include [8], which describes 2 partitional methods, and [18], which is a hierarchical clustering approach. Nahm and Mooney [93] described a methodology where information extraction and data mining can be combined to improve upon each other; information extraction provides the data mining process with access to textual documents (text mining) and in turn data mining provides learned rules to the information extraction portion to improve its performance.

\subsection{Traditional Information Retrieval Techniques for Plain-Text Documents}

Traditional information retrieval methods represent plain-text documents using a series of numeric values for each document. Each value is associated with a specific term (word) that may appear on a document, and the set of possible terms is shared across all documents. The values may be binary, indicating the presence or absence of the corresponding term. The values may also be a non-negative integers, which represents the number of times a term appears on a document (i.e. term frequency). Non-negative real numbers can also be used, in this case indicating the importance or weight of each term. These values are derived through a method such as the popular inverse document frequency $(t f \cdot i d f)$ model [107], which reduces the importance of terms that appear on many documents. Regardless of the method used, each series of values represents a document and corresponds to a point (i.e. vector) in a Euclidean feature space; this is called the vector-space model of information retrieval. This model is often used when applying machine learning techniques to documents, as there is a strong mathematical foundation for performing distance measure and centroid calculations using vectors.

Document clustering using the vector model has long been studied in the information retrieval field as a means of improving retrieval efficiency and corpus visualization [6][107]. For example, in [29] the application described by the authors uses the popular agglomerative hierarchical clustering method to create a cluster hierarchy for the entire document collection for the purpose of visualizing and browsing the document corpus. Another similar approach is that of Kohonen et al. [62], which uses self- 
organizing maps, a type of unsupervised neural network, to group documents in a collection. Like the application described in [29], the Kohonen et al. system provides a environment where the results and groupings can be browsed. Early work on clustering documents retrieved by queries for the purpose of improving user navigation through the results is reported in [55].

A topic related to document clustering is that of document classification. The goal of such a task is to assign a label (or class) to a previously unseen document. This is different from document clustering, where the objective is to create groupings of a document collection. Document classification is a supervised learning task where example documents and their categories are available for learning in advance. Document classification is used for automated (rather than manual) categorization for documents. In [130], Weiss et al. studied the application of decision tree methods in order to categorize text documents. McCallum and Nigam [82] used a Bayesian (probabilistic) approach for document classification.

There are several reasons why information retrieval methods that deal with traditional text documents are not entirely suitable for web content mining. First, web documents contain additional markup elements (HTML tags) which are not found in plain-text documents. These elements can be a source of additional knowledge about the documents. As we saw above, there is a branch of web mining (web structure mining) that attempts to exploit the hyperlink information found in web documents. This information is not available for plain-text documents, so we must find ways to incorporate this information into our data representations during web mining. This is a major limitation of existing web content mining methods, as they either require discarding such information to arrive at traditional plain-text vector representations or they necessitate new or altered data mining procedures which explicitly take the additional information into account. Second, the web is highly heterogeneous, especially when compared to document corpora that are related to a single topic or field of interest. For example, the term "Amazon" can refer to many things on the Internet: an on-line book store, a rain forest, a river, or a person. Thus we may be unable to use specific domain knowledge (such as specialized stop word or synonym lists) that we could otherwise utilize in a system developed for a well-defined, homogeneous document collection. Additionally, web documents have a wide variation in size, style, layout, languages, etc. We must deal with these variations. Third, traditional information retrieval methods may not scale well to the size or dynamic nature of the Internet. Web pages tend to change often with time (they are updated, they are moved to another location, etc.) and thus techniques used in traditional information retrieval systems, such as those related to generation of indices or representatives of documents, can lead to outof-date results. The web contains hundreds of millions of pages, some of which change frequently and must be re-examined periodically. These last two points are especially problematic for web document categorization methods, since creating an adequate training set to encompass all desired categories and updating it to deal with changing conditions is extremely difficult.

In contrast to the methods mentioned above, the work presented here represents the first time web document content itself has been modeled and retained using graphs in a web mining method. Note that, as we mentioned above, graph representations have 
been used for web mining (e.g. web graphs in web structure mining). However, the difference is that those graphs represent the documents (nodes) and their relationships through hyperlinks (edges). Our graphs represent the textual content of web documents through words (nodes) and adjacency information (edges), as will be discussed in detail in Chapter 4. Only recently have a few papers appeared in the literature that deal with representing the web documents themselves using graphs. Lopresti and Wilfong compared web documents using a graph representation that primarily utilizes HTML parse information, in addition to hyperlink and content order information [72]. In their approach they use graph probing, which extracts numerical feature information from the graphs, such as node degrees or edge label frequencies, rather than comparing the graphs themselves. In contrast, our representation uses graphs created solely from the content, and we utilize the graphs themselves to determine document similarity rather than a set of extracted features. Liang and Doermann represent the physical layout of document images as graphs [70]. In their layout graphs nodes represent elements on the page of a document, such as columns of text or headings, while edges indicate how these elements appear together on the page (i.e. they capture the spatial relationships). This method is based on the formatting and appearance of the documents when rendered, not the textual content (words) of a document as in our approach. Another recently reported approach [33][101] takes both the content and structure of web documents into account. Documents are initially represented by graphs according to either naturally occurring elements (e.g. pages, sections, paragraphs, etc.) or XML markup structure. However, rather than manipulating the graphs directly as data, as is done in our approach, probabilistic information is extracted to create Bayesian networks for retrieval or classification. Thus this method is more similar to a probabilistic learning model, rather than a purely graph-theoretical one.

\subsection{Web Search Clustering}

In Chapter 5 we will introduce a system we have created for the purpose of clustering search results returned by conventional search engines. The goal is to separate the results into groups of topics in order to allow the user to more easily find the desired web pages. Web page clustering as performed by humans was examined by Macskassy et $a l$. [77]. Ten subjects were involved in the experiments, and each was asked to manually cluster the results of five different queries submitted to a web search engine at Rutgers University. The queries were selected from the most popular submitted to this particular web search engine: accounting, career services, employment, library, and off campus housing. All subjects received the pages' URLs and titles, however four of the ten subjects were also given the full text of each page for each query. The subjects then clustered the group of documents associated with each query. The investigators examined the size of clusters created, the number of clusters created, the similarity of created clusters, the amount of cluster overlap, and documents not clustered. The results indicated that the size of clusters was not affected by access to the full text of each document and that there was no preference for a specific cluster size. As to number of clusters, the subjects who had access to the full text of the web pages tended to create more clusters than those who did not. 
There are some important papers that deal specifically with the clustering of web search results using automated systems. One of the earliest such papers is that of Zamir and Etzioni [139], which gives some of the first experimental evaluations of clustering methods as applied to web search results. In that paper the authors introduced a clustering algorithm called suffix tree clustering (or STC) in order to cluster web search results. STC works by creating a tree of words encountered in the web documents such that documents that share words or strings of words also share a common parent node in the tree. The nodes in this tree are then scored according to the number of documents represented by the node multiplied by a weighting factor. Based on this score a cluster similarity is determined, which is used to determine cluster overlap and the merging of overlapping clusters. The result is a group of clusters which have terms or phrases related with them. STC was used in the authors' Grouper system to implement a system for clustering web search results. STC was also used in a recently reported system called Carrot $^{2}$, which was used to cluster both English and Polish documents [119]. We will compare our system to the Grouper system in Chapter 5. Unfortunately, it is not possible to directly compare our system to the experimental results of these papers, as the data sets used are not available to the public. A similar web search system, called MSEEC, was introduced in [51]. MSEEC uses the LZW compression algorithm to generate a tree similar to that of STC. An interesting feature of MSEEC is that it can utilize multiple search engines. This can be useful if, for example, the search engines cover different sets of documents. An updated version of our system also made use of multiple search engines, as did the system of [25]. No experimental results or comparisons were presented in [51], only the system architecture and relevant algorithms. The on-line versions of both MSEEC and Grouper appear to be non-functional as of this writing, limiting us to using the results we had already gathered previously. Another web search clustering system is described by He et al. [54]. This system uses a hybrid web structure/web content mining approach to clustering, by creating a graph of web pages based on their hyperlinks and then performing a graph partitioning method. Textual information from the pages, in the form of vector representations, is used to determine the weight of edges in the graph. Cocitation, where we assume that when many pages link to the same target pages this implies that the target pages are related, is also used in the calculation of edge weights. This system was not available to the public, nor was the data used in the experiments. Organizing web search results using classifiers has also been studied [36], however these methods require supervised learning using a fixed group or hierarchy of clusters. If new topics are to be created, as often happens with the highly dynamic nature of the Internet, the classifier training process must be repeated.

From personal experience in maintaining such a system we know that constant updates are required as search engine sites periodically change the format that their results are presented in. Thus many academic-based systems tend to go off-line after a time. There are some web search clustering systems that are developed with commercial, rather than academic, interests in mind. These systems are usually not reported in the scientific literature (except in reviews of such systems). An example of one such system is Vivísimo [125]. Unfortunately, because the developers often wish to keep the inner workings of such systems confidential, it is impossible to examine the algorithms used to the perform clustering. Further, because such systems may be frequently updated (e.g. to 
improve performance or accommodate changes in search engines), it is extremely difficult to perform a stable, large, long-term comparison with these systems. In this dissertation we present a comparison of our system to both Grouper (which is a no longer functional academically reported system) and Vivísimo (a commercial system) in Chapter 5, using previously captured results.

Another important paper that is strongly related to the current work is that of Strehl et al. [120], which examined clustering performance of different clustering algorithms when using various similarity measures on web document collections. Clustering methods examined in the paper included $k$-means, graph partitioning, and selforganizing maps (SOMs). Vector-based representations were used in the experiments along with distance measures based on Euclidean distance, cosine similarity, and Jaccard similarity. Fortunately, one of the data sets used in this paper is publicly available and we will use it in our experiments in Chapter 6, where we compare the performance of our $k$ means clustering method using graph representations to the results presented by Strehl et al., which use vector representations.

\subsection{Summary}

Above we mentioned a major drawback of existing web mining methods is that they either require using a vector representation (as with plain-text documents, which discards structural and web-related information) or they must develop new algorithms and frameworks for handling the additional web-related information. Our main novel contribution in this dissertation is the use of graphs for representing web document content during web mining and extending existing, proven machine learning algorithms to operate on these representations. This has not been done before because previously no mathematical foundation existed for dealing directly and efficiently with graphs in this way. Using graphs instead of vectors is extremely beneficial because it allows structural information that is often lost when using simpler models (such as term appearance order or section information) to be maintained during web mining. This additional information improves the performance of web document clustering and classification. For a web search clustering system, graphs have the additional benefit of preserving the term order information, which provides the user with a more coherent display of cluster labels. Since we are performing straightforward extensions to existing machine learning methods which allow them to utilize graphs, rather than creating a new framework specifically for web documents, we are not tied to any specific graph representation of a web document. In fact, we will examine several different ways of representing web documents as graphs, all of which utilize the same algorithms. As long as we can model our data using graphs, we can apply our techniques to it. This leads to a more general and flexible methodology, one which is applicable to domains outside of web mining.

In the next chapter we will continue our literature review by looking at techniques for performing graph-theoretic similarity, which forms the foundation for the mathematical framework we will use in our web mining techniques. 


\section{Chapter Three}

\section{A Review of Graph Similarity Techniques}

\subsection{Introduction}

We will use the concepts of graph similarity, graph distance, and graph matching in the following chapters as they form a basis for the novel approaches we have developed for performing clustering and classification tasks using graphs instead of more restrictive vectors. The purpose of the current chapter is to give a literature survey of the various methods that are used to determine similarity, distance and matchings between graphs as well as introduce the formal notation which will later be necessary to describe our algorithms. These topics are closely related to the topics of inexact graph matching or graph similarity, and several practical applications that utilize graph similarity or graph matching are represented in the literature, many of them in the field of image processing. Haris et al. performed labeling of coronary angiograms with graph matching [53]. In [97] a method for allocating tasks in multiprocessor systems using graphs and graph matching is described. In [57] Huet and Hancock describe a graph matching method for shape recognition in image databases. Another area where graph similarity and matching is popular is in chemistry, due to the natural representation of chemical structures $(e . g$. molecules) as graphs [47][96]. For a recent review of graph matching techniques used in pattern recognition, see [21].

In this dissertation we are specifically interested in using graph techniques for dealing with web document content. Traditional learning methods applied to the tasks of text or document classification and categorization, such as rule induction [4] and Bayesian methods [82], are based on a vector model of document representation or an even simpler Boolean model. Similarity of graphs in domains outside of information retrieval has largely been studied under the topic of graph matching. Under this model there exists a database of graphs and an input (or query) graph; the goal is to find the graph in the database that most closely matches the input graph [67]. In many applications the input graph is not expected to be an exact match to any database graph since the input graph is either previously unseen or assumed to be corrupted with some amount of noise. Thus we sometimes refer to this area as error-tolerant or inexact graph matching. As mentioned above, a number of graph matching applications have been reported in the literature, including the recognition of characters, graphical symbols, and two-dimensional shapes. For a recent survey see [11]. We are not aware, however, of any graph matching applications that deal with content-based categorization and classification of web or text documents.

The remainder of this chapter is organized as follows. First, in Section 3.2, we give the mathematical notations and the definitions of graph and subgraph isomorphisms. 
If two graphs are isomorphic then there is an exact 1-to-1 matching between them; graph isomorphism was the basis for early (exact) graph matching methods. The other basic notation and definitions used in the dissertation will also be given. In Section 3.3, we explain graph edit distance and how it provides a numeric dissimilarity between two graphs. In Section 3.4, we describe the maximum common subgraph approach and explain how it is related to graph edit distance. The state space search method is given in Section 3.5. In Section 3.6, we describe a probabilistic approach to matching graphs with errors. In Section 3.7, we recount a method based on distance preservation between graph nodes. We give some relaxed (sub-optimal) approaches to graph matching in Section 3.8. In Section 3.9, we give an account of mean and medians of a set of graphs; while not measurements of graph similarity per se, these concepts are related and very useful in certain applications. Summary remarks are given in Section 3.10.

\subsection{Graph and Subgraph Isomorphism}

In this section we describe graph and subgraph isomorphism. Before we give definitions for isomorphism, we first give definitions for graph and subgraph. A graph $G$ [13][128] is a 4-tuple: $G=(V, E, \alpha, \beta)$, where $V$ is a set of nodes (also called vertices), $E \subseteq V \times V$ is a set of edges connecting the nodes, $\alpha: V \rightarrow \Sigma_{V}$ is a function labeling the nodes, and $\beta: V \times V \rightarrow \Sigma_{E}$ is a function labeling the edges $\left(\Sigma_{V}\right.$ and $\Sigma_{E}$ being the sets of labels that can appear on the nodes and edges, respectively). For brevity, we may abbreviate $G$ as $G=(V, E)$ by omitting the labeling functions.

A graph $G_{1}=\left(V_{1}, E_{1}, \alpha_{1}, \beta_{1}\right)$ is a subgraph [9] of a graph $G_{2}=\left(V_{2}, E_{2}, \alpha_{2}, \beta_{2}\right)$, denoted $G_{1} \subseteq G_{2}$, if $V_{1} \subseteq V_{2}, E_{1} \subseteq E_{2} \cap\left(V_{1} \times V_{1}\right), \alpha_{1}(x)=\alpha_{2}(x) \forall x \in V_{1}$, and $\beta_{1}((x, y))=\beta_{2}((x, y))$ $\forall(x, y) \in E_{1}$. Conversely, graph $G_{2}$ is also called a supergraph of $G_{1}$.

When we say that two graphs are isomorphic, we mean that the graphs contain the same number of nodes and there is a direct 1-to-1 correspondence between the nodes in the two graphs such that the edges between nodes and all labels are preserved. Formally, a graph $G_{1}=\left(V_{1}, E_{1}, \alpha_{1}, \beta_{1}\right)$ and a graph $G_{2}=\left(V_{2}, E_{2}, \alpha_{2}, \beta_{2}\right)$ are said to be isomorphic [9], denoted $G_{1} \cong G_{2}$, if there exists a bijective function $f: V_{1} \rightarrow V_{2}$ such that $\alpha_{1}(x)=\alpha_{2}(f(x))$ for $\forall x \in V_{1}$ and $\beta_{1}((x, y))=\beta_{2}((f(x), f(y)))$ for $\forall(x, y) \in V_{1} \times V_{1}$. Such a function $f$ is also called a graph isomorphism between $G_{1}$ and $G_{2}$.

There is also the notion of subgraph isomorphism, meaning a graph is isomorphic to a part of (i.e. a subgraph of) another graph. Given a graph isomorphism $f$ between graphs $G_{1}$ and $G_{2}$ as defined above and another graph $G_{3}$, if $G_{2} \subseteq G_{3}$ then $f$ is a subgraph isomorphism [13] between $G_{1}$ and $G_{3}$.

It is not known whether graph isomorphism is an NP-complete problem, however subgraph isomorphism is NP-complete [85][88]. Clearly, as the number of nodes in the graphs increase the number of possible matchings to be checked increases combinatorally. A general procedure for determining subgraph isomorphism is given in [124]. The naïve algorithm for graph isomorphism is to maintain a matrix which indicates which nodes in each graph are compatible; it can require all possible permutations of matchings to determine if there is an isomorphism. The procedure in [124] improves the complexity by pruning the search space. Graph isomorphism tells us only that there exists an exact match between two graphs (i.e. that they are identical). It does not give us any 
indication of similarity between graphs, only that they are isomorphic or not. Subgraph isomorphism tells us if one graph appears as part of another graph. Formally, the similarity between two graphs $G_{1}$ and $G_{2}$, denoted $s\left(G_{1}, G_{2}\right)$, is a function that has the following properties:

(1) $0 \leq s\left(G_{1}, G_{2}\right) \leq 1$

(2) $s\left(G_{1}, G_{2}\right)=1 \rightarrow G_{1} \cong G_{2}$

(3) $s\left(G_{1}, G_{2}\right)=s\left(G_{2}, G_{1}\right)$

(4) if $G_{1}$ is more similar to $G_{2}$ than to $G_{3}$, then $s\left(G_{1}, G_{2}\right) \geq s\left(G_{1}, G_{3}\right)$

One problem with defining similarity in this way is that it is not clear what case causes $s\left(G_{1}, G_{2}\right)=0$. This comes from the fact that we have no concept of an exact "opposite" of a graph. We do, however, have the idea of compliments of graphs. A compliment [22] of a graph $G$, denoted $\bar{G}$, is the fully connected version of $G$ such that the edges in $G$ have been removed, $\bar{E}=\{(u, v) \mid(u, v) \notin E\}$.
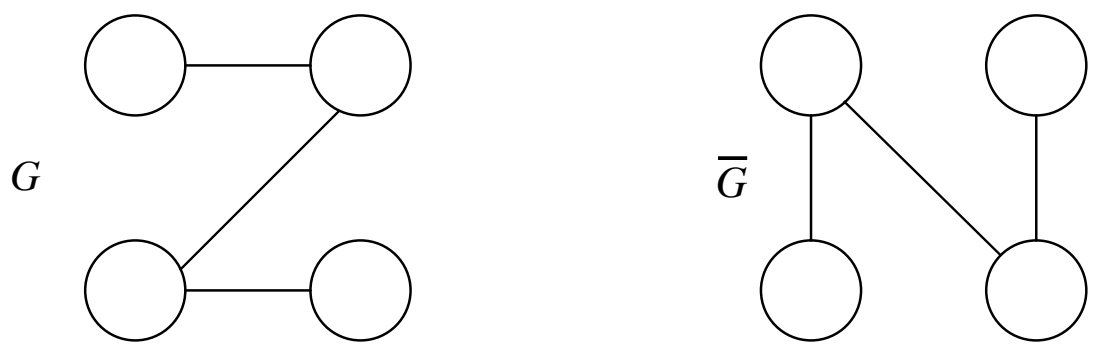

\section{Figure 3.1. A Graph $\mathbf{G}$ and its Compliment $\bar{G}$ May be Isomorphic}

However, a graph may be isomorphic to its compliment (Fig. 3.1), so it does not necessarily hold that $s(G, \bar{G})=0$. Given this limitation, the usual method of determining numeric similarity between graphs is to use a distance measure. A distance metric [13][15][39][127] between two graphs, denoted $d\left(G_{1}, G_{2}\right)$, is a function that has the following properties:

(1) boundary condition: $d\left(G_{1}, G_{2}\right) \geq 0$

(2) identical graphs have zero distance: $d\left(G_{1}, G_{2}\right)=0 \rightarrow G_{1} \cong G_{2}$

(3) symmetry: $d\left(G_{1}, G_{2}\right)=d\left(G_{2}, G_{1}\right)$

(4) triangle inequality: $d\left(G_{1}, G_{3}\right) \leq d\left(G_{1}, G_{2}\right)+d\left(G_{2}, G_{3}\right)$

We note that it is possible to transform a similarity measure into a distance measure, for example by:

$$
d\left(G_{1}, G_{2}\right)=1-s\left(G_{1}, G_{2}\right)
$$

It can be shown that this equation satisfies the various conditions above for similarity. Other equations are also possible for changing distance into similarity. Throughout the 
rest of this dissertation we will see several proposed distance measures, some of which have been created from a similarity measure.

\subsection{Graph Edit Distance}

Edit distance is a method that is used to measure the difference between symbolic data structures such as trees [121] and strings [126]. It is also known as the Levenshtein distance, from early work in error correcting/detecting codes that allowed insertion and deletion of symbols [68]. The concept is straightforward. Various operations are defined on the structures, such as deletion, insertion, and renaming of elements. A cost function is associated with each operation, and the minimum cost needed to transform one structure into the other using the operations is the distance between them. Edit distance has also been applied to graphs, as graph edit distance [108]. The operations in graph edit distance are insertion, deletion, and re-labeling of nodes and edges.

Formally, an editing matching function (or an error correcting graph matching, ecgm [9]) between two graphs $G_{1}$ and $G_{2}$ is defined as a bijective mapping function $M: G_{\mathrm{x}} \rightarrow G_{\mathrm{y}}$, where $G_{\mathrm{x}} \subseteq G_{1}$ and $G_{\mathrm{y}} \subseteq G_{2}$. The following six edit operations on the graphs, which are implied by the mapping $M$, are also defined:

(1) If a node $v \in V_{1}$ but $v \notin V_{\mathrm{x}}$ then we delete node $v$ with cost $c_{\text {nd }}$.

(2) If a node $v \in V_{2}$ but $v \notin V_{\mathrm{y}}$ then we insert node $v$ with $\operatorname{cost} c_{\text {ni }}$.

(3) If $M\left(v_{\mathrm{i}}\right)=v_{\mathrm{j}}$ for $v_{\mathrm{i}} \in V_{\mathrm{x}}$ and $v_{\mathrm{j}} \in V_{\mathrm{y}}$ and $\alpha_{1}\left(v_{\mathrm{x}}\right) \neq \alpha_{2}\left(v_{\mathrm{y}}\right)$ then we substitute node $v_{\mathrm{i}}$ with node $v_{\mathrm{j}}$ with $\cos t c_{\mathrm{ns}}$.

(4) If an edge $e \in E_{1}$ but $e \notin E_{\mathrm{x}}$ then we delete edge $e$ with cost $c_{\text {ed }}$.

(5) If an edge $e \in E_{2}$ but $e \notin E E_{\mathrm{y}}$ then we insert edge $e$ with $\operatorname{cost} c_{\mathrm{ei}}$.

(6) If $M\left(e_{\mathrm{i}}\right)=e_{\mathrm{j}}$ for $e_{\mathrm{i}} \in E_{\mathrm{x}}$ and $e_{\mathrm{j}} \in E_{\mathrm{y}}$ and $\beta_{1}\left(e_{\mathrm{x}}\right) \neq \beta_{2}\left(e_{\mathrm{y}}\right)$ then we substitute edge $e_{\mathrm{i}}$ with edge $e_{\mathrm{j}}$ with cost $c_{\mathrm{es}}$.

We note that there may be several functions $M$ which edit graph $G_{1}$ into graph $G_{2}$. The cost [9][10] of a given an editing function $M$, denoted $\gamma(M)$, is defined as the sum of the costs $c$ of all the individual edit operations implied by $M$. Usually the cost coefficients $c$ are application dependant. In the error correcting graph matching sense, they can be related to the probability of the operations (errors) occurring. We assume that the cost coefficients are non-negative and are invariant of the node or edge upon which they are applied (i.e. the costs are constant for each operation).

The edit distance between two graphs [9], denoted $d\left(G_{1}, G_{2}\right)$, is defined as the cost of the mapping $M$ that results in the lowest $\gamma(M)$. More formally:

$$
d\left(G_{1}, G_{2}\right)=\min _{\forall M}(\gamma(M))
$$

Thus the distance between two graphs is the cost of an editing function which transforms one graph into the other via edit operations and which has the lowest cost among all such editing functions.

The advantage to the graph edit distance approach is that it is easy to understand and straightforward to apply. The disadvantage is that the costs for the edit operations (6 
parameter values) need to be determined for each application. In [10], Bunke gives an examination of cost functions for graph edit distance. He shows that an infinite number of equivalent cost classes exist under certain conditions; i.e., it is the ratios between the different edit costs that differentiates sets of cost functions, rather than the individual cost values.

\subsection{Maximum Common Subgraph / Minimum Common Supergraph Approach}

Bunke has shown [9] that there is a direct relationship between graph edit distance and the maximum common subgraph between two graphs. Specifically, the two are equivalent under certain restrictions on the cost functions. A graph $g$ is a maximum common subgraph (mcs) [9] of graphs $G_{1}$ and $G_{2}$, denoted $m c s\left(G_{1}, G_{2}\right)$, if: (1) $g \subseteq G_{1}$ (2) $g \subseteq G_{2}$ and (3) there is no other subgraph $g^{\prime}\left(g^{\prime} \subseteq G_{1}, g^{\prime} \subseteq G_{2}\right)$ such that $|g|>|g|$. (Here $|g|$ is usually taken to mean $|V|$, i.e. the number of nodes in the graph; it is used to indicate the "size" of a graph.)

Similarly, there is the complimentary idea of minimum common supergraph. A graph $g$ is a minimum common supergraph (MCS) [13] of graphs $G_{1}$ and $G_{2}$, denoted $\operatorname{MCS}\left(G_{1}, G_{2}\right)$, if: (1) $G_{1} \subseteq g$ (2) $G_{2} \subseteq g$ and (3) there is no other supergraph $g^{\prime}\left(\mathrm{G}_{1} \subseteq g^{\prime}, \mathrm{G}_{2} \subseteq g\right.$ ) such that $|g|<|g|$.

Methods for determining the $m c s$ are given in [69][83]. The general approach is to create a compatibility graph for the two given graphs, and then find the largest clique within it. What Bunke has shown is that when computing the editing matching function based on graph edit distance (Section 3.3), the function with the lowest cost is equivalent to the maximum common subgraph between the two graphs under certain conditions on the cost coefficients. This is intuitively appealing, since the maximum common subgraph is the part of both graphs that is unchanged by deleting or inserting nodes and edges. To edit graph $G_{1}$ into graph $G_{2}$, one only needs to perform the following steps:

(1) Delete nodes and edges from $G_{1}$ that don't appear in $m c s\left(G_{1}, G_{2}\right)$

(2) Perform any node or edge substitutions

(3) Add the nodes and edges from $G_{2}$ that don't appear in $m c s\left(G_{1}, G_{2}\right)$

Following this observation that the size of the maximum common subgraph is related to the similarity between two graphs, Bunke and Shearer [15] have introduced a distance measure based on $m c s$. They defined the following distance measure:

$$
d_{M C S}\left(G_{1}, G_{2}\right)=1-\frac{\left|\operatorname{mcs}\left(G_{1}, G_{2}\right)\right|}{\max \left(\left|G_{1}\right|,\left|G_{2}\right|\right)}
$$

where $\max (x, y)$ is the usual maximum of two numbers $x$ and $y$, and I...I indicates the size of a graph (usually taken to be the number of nodes in a graph). This distance measure has four important properties [13] (see Section 3.2 above). First, it is restricted to producing a number in the interval $[0,1]$. Second, the distance is 0 only when the two graphs are identical. Third, the distance between two graphs is symmetric. Fourth, it obeys the triangle inequality, which ensures the distance measure behaves in an intuitive 
way. For example, if we have two dissimilar objects (i.e. there is a large distance between them) the triangle inequality implies that a third object which is similar (i.e. has a small distance) to one of those objects must be dissimilar to the other. The advantage of this approach over the graph edit distance method is that it does not require the determination of any cost coefficients or other parameters. However, the metric as it is defined in Eq. 3.3 may not be appropriate for all applications. Thus several other distance measures based on the size of the maximum common subgraph or minimum common supergraph have been proposed.

A second distance measure which has been proposed by Wallis et al. [127], based on the idea of graph union, is:

$$
d_{W G U}\left(G_{1}, G_{2}\right)=1-\frac{\left|m c s\left(G_{1}, G_{2}\right)\right|}{\left|G_{1}\right|+\left|G_{2}\right|-\left|m c s\left(G_{1}, G_{2}\right)\right|}
$$

By "graph union" we mean that the denominator represents the size of the union of the two graphs in the set theoretic sense; specifically adding the size of each graph $\left(\left|G_{1}\right|+\left|G_{2}\right|\right)$ then subtracting the size of their intersection $\left(\left|m c s\left(G_{1}, G_{2}\right)\right|\right)$ leads to the size of the union (the reader may easily verify this using a Venn diagram). A similar distance measure [9] which is not normalized to the interval $[0,1]$ is:

$$
d_{U G U}\left(G_{1}, G_{2}\right)=\left|G_{1}\right|+\left|G_{2}\right|-2\left|m c s\left(G_{1}, G_{2}\right)\right|
$$

Fernández and Valiente have proposed a distance measure based on both the maximum common subgraph and the minimum common supergraph [39]:

$$
d_{M M C S}\left(G_{1}, G_{2}\right)=\left|M C S\left(G_{1}, G_{2}\right)\right|-\left|m c s\left(G_{1}, G_{2}\right)\right|
$$

where $\operatorname{MCS}\left(G_{1}, G_{2}\right)$ is the minimum common supergraph of graphs $G_{1}$ and $G_{2}$.

We can also create a version of this distance measure which is normalized to $[0,1]$ as follows:

$$
d_{M M C S N}\left(G_{1}, G_{2}\right)=1-\frac{\left|\operatorname{mcs}\left(G_{1}, G_{2}\right)\right|}{\left|\operatorname{MCS}\left(G_{1}, G_{2}\right)\right|}
$$

For brevity we will refer to the distance measures of Eqs. 3.3-3.7 as MCS, WGU, UGU, MMCS, and MMCSN, respectively.

The distance metrics of Eqs. 3.3 to 3.7 are relatively new, and not much has been reported regarding their differences in performance for different problem domains. We will examine this issue in the coming chapters. Also, the graph size $|G|$ is typically defined simply as the number of nodes in the graph; the edge information is not (explicitly) captured in these distance measures.

Note that if the condition holds that $\left|M C S\left(G_{1}, G_{2}\right)\right|=\left|G_{1}\right|+\left|G_{2}\right|-\left|m c s\left(G_{1}, G_{2}\right)\right|$, then WGU and MMCSN are identical. Similarly, UGU and MMCS are identical. This can be verified by substituting this definition for $\left|M C S\left(G_{1}, G_{2}\right)\right|$ into Eqs. 3.6 and 3.7. 


\subsection{State Space Search Approach}

In Section 3.3 we described the graph edit distance approach for determining graph similarity. In order to find the distance we need to find an edit matching function that has the lowest cost for the given cost coefficients. Depending on the size of the graphs and the costs associated with the edit operations, finding the lowest cost mapping may require an exhaustive examination of all possible matchings. If we allow the possibility of not having to determine the exact distance between graphs, we can perform other types of sub-optimal search. These searches may not find the global minimum cost function, but they can be performed more quickly (since we do not need to find all of the possible matching functions) and still yield acceptable results.

Each matching function we consider becomes a state in a search space. The cost $\gamma(M)$ for a state $M$ becomes the value we attempt to minimize through the search. $M$ is actually a graph isomorphism between subgraphs of the two graphs being matched; it specifies the operations needed to edit one graph into the other graph. Neighbors of a state $M$ can be determined by adding/deleting nodes and edges to/from these subgraphs along with their corresponding isomorphic matching; these neighbor states indicate the creation (or removal) of a single matching between a node or edge in the two graphs (i.e. it specifies a change in the edit operations). Once the matching is represented in such a manner, many techniques become available for performing the search, including hill climbing, genetic algorithms, simulated annealing, and so forth. These searches may not find the optimal solution, but for some applications (such as graph matching for retrieval of images or documents) this may not be a concern. These techniques are also sensitive to initialization and parameter selection, so there can be a wide variety in performance.

For a more detailed description of this technique as well as experimental results comparing different search and initialization strategies, we refer the reader to [128]. Early work in this area can be found in [38].

\subsection{Probabilistic Approach}

In this section we will give a summary of the approach proposed by Wilson and Hancock [133] which is based on probability theory. In the probabilistic method, we attempt to match a data graph $G_{\mathrm{D}}$ and a stored model graph $G_{\mathrm{M}}$. These graphs are attributed graphs. An attributed graph [133] is a graph $G_{y}=(V, E, A)$, where $A$ is a set of attributes associated with each node, $A=\left\{x_{v}^{y}, \forall v \in V\right\}$.

The attributes in the data graph are to be matched to those in the model graph, such that the matched nodes have the same or similar attributes. Edges may also have associated attributes in this model, but they are not considered in this approach. Next, we have the concept of super-clique of a node. A super-clique [133] of a node $i$ in graph $G=(V, E)$ is defined as $C_{i}=i \cup\{j \mid(j, i) \in E\}$. In other words, the super-clique of a node $i$ is the set of nodes which contains $i$ and all nodes connected to it by edges. We attempt to match all super-cliques in the data graph with super-cliques in the model graph.

The set of all possible matches between super-clique $C_{i}$ in the data graph $G_{\mathrm{D}}$ and super-cliques $S_{j}$ in the model graph $G_{\mathrm{M}}$ is called a dictionary [133] and is denoted $\Theta_{i}$. To cope with size differences between the data and model super-cliques we allow dummy 
(or null) nodes $\phi$ to be inserted into $S_{j}$ so that both graphs have equal numbers of nodes. The function matching a node in $C_{i}$ to a node in $S_{j}$ is $f: V_{\mathrm{D}} \rightarrow V_{\mathrm{M}} \cup \phi$. The probability of matching errors (a node in the data graph is matched to the wrong node in the model graph) is denoted $P_{e}$ and the probability of structural errors (a node in the data graph is matched to a dummy node in the model graph) is denoted $P_{\phi}$. Given these definitions, some assumptions, and through application of Bayes' rule and other probability theoretic constructions, Wilson and Hancock arrive at a mathematical description for the probability of a super-clique matching between two graphs (denoted $\Gamma_{j}$ for super-clique $\left.C_{j}\right)$ :

$$
P\left(\Gamma_{j}\right)=\frac{K_{C_{j}}}{\left|\Theta_{j}\right|} \sum_{S_{j} \in \Theta_{j}} \exp \left\{-\left(k_{e} H\left(\Gamma_{j}, S_{i}\right)+k_{\phi}\left[\psi\left(\Gamma_{j}, S_{i}\right)+\Psi\left(\Gamma_{j}\right)\right]\right)\right\}
$$

where

$$
\begin{gathered}
K_{C_{j}}=\left[\left(1-P_{e}\right)\left(1-P_{\phi}\right)\right]^{\left|C_{j}\right|} \\
k_{e}=\ln \frac{1-P_{e}}{P_{e}} \\
k_{\phi}=\frac{\left(1-P_{e}\right)\left(1-P_{\phi}\right)}{P_{\phi}}
\end{gathered}
$$

$H\left(\Gamma_{j}, S_{i}\right)$ is the Hamming distance between the super-clique of the data graph under the mapping $f$ and the super-clique of the model graph, $\psi\left(\Gamma_{j}, S_{i}\right)=\left|C_{j}\right|-\left|S_{i}\right|$ (i.e. the number of null nodes inserted into $\left.S_{i}\right)$, and $\Psi\left(\Gamma_{j}\right)$ is the number of nodes in $C_{j}$ which are mapped onto null nodes in $S_{i}$. The derivation of Eq. 3.8 is beyond the scope of this chapter, but the equation contains three parts which are fairly straightforward. First, the part associated with Eq. 3.9 is the probability of no errors occurring. Second, the part associated with Eq. 3.10 is concerned with the probability of matching errors occurring. Third, the part associated with Eq. 3.11 deals with the probability of structural errors occurring. For an in depth derivation of these equations, please refer to [133].

The authors then go on to derive rules which can be applied to update the matching function $f$ under three different methods (null-labeling, constraint filtering, and graph edit operations). The methods use update rules of the form:

$$
f(u)=\arg \max _{v \in V_{M}} \frac{P\left(u, v \mid x_{u}^{D}, x_{v}^{M}\right)}{P(u, v)} \sum_{j \in C_{u}} P\left(\Gamma_{j}\right)
$$

Here $P(u, v)$ indicates the prior probability that node $u$ in the data graph corresponds to node $v$ in the model graph, while the other probability in the numerator is the conditional a posteriori probability given the corresponding attributes related with the nodes; 
approaches to determining these probabilities are application dependant. One method uses $\mathrm{P}\left(\Gamma_{j}\right)$ as in Eq. 3.8, while the others use the form

$$
P\left(\Gamma_{j}\right)=\sum_{S_{i} \in \Theta_{j}} e^{-k_{e} H\left(\Gamma_{j}, S_{i}\right)}
$$

This form is obtained by setting the parameter $k_{\phi}=0$, in other words ignoring the effect of mapping nodes in the data graph to null-labeled nodes in the model graph.

A benefit of this approach is that, under the simpler model of Eq. 3.13, there is only one parameter that must be adjusted. Another advantage of this framework is that it can be applied in many situations. For example, an extension of the work [131] deals with multiple graph matching (compared to data-model graph matching of attributed graphs) through computations of fuzzy consistency matrices. In [40], Finch et al. developed an energy function for graph matching based on the probabilistic framework of this section. A method using this approach for the fitness function in a genetic search for graph matching is described in [28]. A similar probabilistic framework for hierarchical graphs is given in [134]. Myers et al. [92] modified the approach described here to include graph edit distance; the new method achieves better complexity by removing the need to insert null nodes in the model graph.

\subsection{Distance Preservation Approach}

In [19], Chartrand et al. describe an approach for graph distance calculation based on preserving the distance between nodes. The idea comes from the fact that when two graphs are isomorphic, the distances (meaning in this context the number of edges traversed) between every pair of nodes are identical in both graphs. Given a graph $G=(V, E)$, the distance between two nodes $x, y \in V$, denoted $d_{G}(x, y)$, is defined as the minimum number of edges that need to be traversed when traveling from $x$ to $y$ [19]. Further, the $\phi$-distance [19] between two graphs $G_{1}$ and $G_{2}$, denoted $d_{\phi}\left(G_{1}, G_{2}\right)$, is defined as

$$
d_{\phi}\left(G_{1}, G_{2}\right)=\sum_{\forall x \forall y \in V_{1}}\left|d_{G_{1}}(x, y)-d_{G_{2}}(x, y)\right|
$$

where $\phi$ is a 1-to- 1 mapping (but not necessarily an isomorphism) between $G_{1}$ and $G_{2}$. Here $\mid . . . \mathrm{I}$ is the standard absolute value operation.

If $\phi$ is an isomorphism (i.e. $\left.G_{1} \cong G_{2}\right)$, then $d_{\phi}\left(G_{1}, G_{2}\right)=0$; if $G_{1}$ and $G_{2}$ are not isomorphic, then $d_{\phi}\left(G_{1}, G_{2}\right)>0$. This leads to a definition of distance between two graphs, denoted $d\left(G_{1}, G_{2}\right)$, which is formally denoted

$$
d\left(G_{1}, G_{2}\right)=\min _{\forall \phi}\left(d_{\phi}\left(G_{1}, G_{2}\right)\right)
$$

where $\min (\ldots)$ is the minimum function [19]. 
Here again we see the idea of examining all the possible matching functions ( $\phi$, in the notation of the current method; $M$ in the notation of graph edit distance) between two graphs in order to determine the distance between them. Eq. 3.15 above is directly comparable to Eq. 3.2 of Section 3.3, even though these two methods have different theoretical foundations. The authors also go on to show if the graphs meet certain requirements then we can make some other, less expensive calculations. For example, if $G_{1}$ and $G_{2}$ are connected graphs with equal numbers of nodes, then we can determine the lower bound on their distance by

$$
d\left(G_{1}, G_{2}\right) \geq\left|t d\left(G_{1}\right)-t d\left(G_{2}\right)\right|
$$

where

$$
t d(G)=\sum_{\forall u, v \in V} d(u, v)
$$

Or, in other words, the sum of distances between all pairs of nodes in a graph. Further theoretical contributions related to this approach can be found in [19].

\subsection{Relaxation Approaches}

As we mentioned in Section 3.2, some early algorithms for determining exact graph matching (isomorphism) used a matching matrix $(M)$ which indicates the compatibility of nodes in the two graphs being matched. If the $i$ th row and $j$ th column element of $M$, denoted $M_{i j}$, is a 1 , then node $i$ in graph $G_{1}$ is matched with node $j$ in graph $G_{2}$; otherwise there is no match and $M_{i j}=0$. There are constraints on the matrix $M$ so that each row has exactly one 1 and no column has more than one 1. Such a representation and the algorithms applied to it for determining graph matching are straightforward, however they can require generating all the permutations of possible node matchings over the matrix.

In order to improve time complexity, we can instead attempt to approximate the optimal solution by finding good sub-optimal solutions instead. A method that is sometimes used to do this for graph matching problems is called relaxation (or more specifically, discrete relaxation). Put simply, discrete relaxation is a method of transforming a discrete representation (such as the matrix $M$ used for graph matching) into a continuous representation. Thus we can transform a discrete optimization problem (exact graph matching using discrete matrix $M$ ) into a continuous optimization problem. Compared to the state space search approach (Section 3.5), relaxation is a non-linear optimization approach. Gold and Rangarajan [43] applied relaxation to the graph matching problem. They have posed the problem of attributed graph matching in terms of an optimization problem:

$$
E=-\frac{1}{2} \sum_{a=1}^{\left|V_{1}\right|} \sum_{i=1}^{\left|V_{2}\right|} \sum_{b=1}^{\left|V_{1}\right|} \sum_{j=1}^{\left|V_{2}\right|} M_{a i} M_{b j} \sum_{r=1}^{R} C_{a i b j}^{(2, r)}+\alpha \sum_{a=1}^{\left|V_{1}\right|} \sum_{i=1}^{\left|V_{2}\right|} M_{a i} \sum_{s=1}^{S} C_{a i}^{(1, s)}
$$


Here $M$ is the matching matrix as before, $R$ is the number of edge types, $S$ is the number of node types, $\alpha$ is a weighting parameter, and the $C$ s are compatibility measures between the edges of the two graphs. The goal is then to minimize the objective function given in Eq. 3.18. The authors use the graduated assignment algorithm to find an $M$ which minimizes $E$. The general procedure of the algorithm is as follows:

(1) Start with some valid initial matrix $M^{0}$.

(2) Determine a first order Taylor expansion of $M^{0}$ yielding:

$$
Q_{a i}=-\frac{\partial E}{\partial M_{a i}^{0}}=\sum_{b=1}^{\left|V_{1}\right|} \sum_{j=1}^{V_{2} \mid} M_{b j}^{0} C_{a i b j}
$$

(3) Use relaxation to create a continuous representation of $M^{0}$

$$
M_{a i}^{0}=e^{\beta Q_{a i}}
$$

where $\beta$ is a control parameter that is slowly increased as the procedure runs.

(4) Update the matrix $M$ by a normalization procedure over both rows and columns.

(5) Repeat until convergence or iteration limit reached.

Medasani et al. [85] gave a procedure based on fuzzy assignments and relaxation similar to the method just described. The objective function for this approach is

$$
J(M, C)=\sum_{i=1}^{\left|V_{1}\right|+1} \sum_{j=1}^{\left|V_{2}\right|+1} M_{i j}^{2} f\left(C_{i j}\right)+\eta \sum_{i=1}^{\left|V_{1}\right|+1} \sum_{j=1}^{V_{2} \mid+1} M_{i j}\left(1-M_{i j}\right)
$$

where $M$ is now a fuzzy membership matrix $\left(0 \leq M_{i j} \leq 1\right)$ that relates the degree of match between nodes, $C$ is a compatibility matrix between nodes (rather than edges as above), $\eta$ is a control parameter, and

$$
f\left(C_{i j}\right)=e^{-\beta C_{i j}}
$$

The summations in Eq. 3.21 are under the constraint that $(i, j) \neq\left(\left|V_{1}\right|+1,\left|V_{2}\right|+1\right)$; the extra nodes in the graphs are dummy nodes similar to slack variables. The authors then go on to derive the necessary update equations for $M$ and $C$ in order to minimize $J(M, C)$ and propose an algorithm which updates these matrices in an alternating fashion.

A drawback to these methods is that they can get stuck in local minima and are sensitive to initialization. The main benefit, as compared to state space search, is that the equations and algorithms for these methods are derived directly from the objective functions as opposed to performing a general search strategy or having to come up with some heuristics for the search. 


\subsection{Mean and Median of Graphs}

In addition to the graph matching approaches we have described, we should also mention the concepts of mean and median of a set of graphs [50]. These do not explicitly give us an indication of graph similarity, but are useful in summarizing a group of graphs. This is useful in applications such as clustering, where we need to represent a group of graphs by some exemplar graph that represents the cluster.

The mean of two graphs [50] $G_{1}$ and $G_{2}$ is a graph $g$ such that:

$$
d\left(G_{1}, g\right)=d\left(G_{2}, g\right)
$$

and

$$
d\left(G_{1}, G_{2}\right)=d\left(G_{1}, g\right)+d\left(g, G_{2}\right)
$$

In other words, a mean of two graphs $G_{1}$ and $G_{2}$ is a graph $g$ that is equidistant from $G_{1}$ and $G_{2}$ and which is a distance from $G_{1}$ or $G_{2}$ equal to half the distance between $G_{1}$ and $G_{2}$. Clearly the mean will depend on the distance functions chosen, and there may be more than one graph satisfying these conditions; it is also possible that no mean exists for a given pair of graphs.

The weighted mean of two graphs [12] $G_{1}$ and $G_{2}$ is a graph $g$ such that:

$$
d\left(G_{1}, G\right)=\alpha d\left(G_{1}, G_{2}\right)
$$

and

$$
d\left(G_{1}, G_{2}\right)=\alpha d\left(G_{1}, G_{2}\right)+d\left(g, G_{2}\right)
$$

where $0<\alpha<1$. If $\alpha=0.5$, then the same mean as in Eqs. 3.23 and 3.24 results.

An algorithm for finding the weighted mean of two graphs is given in [12]. The method involves finding a subset of editing operations (given the lowest cost editing function between the graphs) for the given $\alpha$ in order to determine the mean graph. In [14], a theoretical proof is given that any graph $g$ such that $\operatorname{mcs}\left(G_{1}, G_{2}\right) \subseteq g \subseteq G_{1}$ or $\operatorname{mcs}\left(G_{1}, G_{2}\right) \subseteq g \subseteq G_{2}$ is a mean of $G_{1}$ and $G_{2}$. Thus the problem becomes finding a graph that is a supergraph of the maximum common subgraph, but a subgraph of one of the original graphs. Finally, we have the concept of the median of a set of graphs, which acts like a representative of the set. The median of a set of graphs $S$ [12] is a graph $g \in S$ such that $g$ has the lowest average distance to all elements in $S$ :

$$
g=\arg \min _{\forall s \in S}\left(\frac{1}{|S|} \sum_{i=1}^{|S|} d\left(s, G_{i}\right)\right)
$$


Since $g \in S$, it is straightforward (and relatively inexpensive) to simply compute the average distance to all graphs for each graph in $S$. Further, the median of a set of graphs always exists; it may or may not also be a mean.

\subsection{Remarks}

In this chapter we have given a survey of the most popular methods for determining graph similarity that are represented in the literature. Graph isomorphism finds an exact 1-to-1 matching between identical graphs and was the earliest approach to graph matching. Unfortunately, it cannot handle inexact graph matching. Graph edit distance is a popular approach that can deal with inexact matching. It determines the cost of a sequence of edit operations needed to transform one graph into another; this cost is the distance between the two graphs. This is a straightforward method, but it requires the determination of several parameters (the costs of the various edit operations). The size of the minimum common subgraph of a pair of graphs has been shown to be related to the graph edit distance. Thus several distance measures that use the size of the minimum common subrgaph have been proposed. This technique has the advantage that edit costs do not need to be determined. However, the computation of the minimum common subgraph is NP-Complete. Another method for calculating graph distance is a distance preservation approach that determines the minimum number of edges (distances) between every pair of nodes in each graph. If the graphs are the same, the distances will be identical for some matching of nodes. Otherwise, there will be a difference indicating the distance between the graphs. This method is only applicable to graphs with equal numbers of nodes and does not appear to be widely used.

Methods such as state space search and relaxation have also been applied to the problem of determining graph similarity. These techniques are often used to provide a sub-optimal approximation when the original problem is NP-Complete or has a high potential for combinatorial explosion. For example, state space search can be used if we represent the matching or edit sequences between graphs as states, and then execute a search strategy for the state with the lowest cost. This results in a sub-optimal version of graph edit distance. Relaxation can be used to transform the discrete problem of node matching between graphs to a continuous representation, which then allows the application of non-linear optimization methods. This creates a sub-optimal version of the graph isomorphism approach. Drawbacks of these two methods are that they can be sensitive to initialization or become trapped at local optima. Another methodology which has been applied to the problem of matching attributed graphs is a probabilistic approach. There are several applications and extensions relating to this method, but the procedure is not straightforward and is only applicable to attributed graphs.

As we have seen, the approaches often have similarities with one another. For example, probability can be seen not just in the Bayesian approach described in Section 3.6, but also in the cost functions of graph edit distance (Section 3.2) and some state space search approaches (Section 3.5). Both state space representations and relaxation attempt to find good sub-optimal solutions. The idea of a function mapping (or transforming) one graph to another can be found in isomorphism, graph edit distance, state space representation, and minimum common subgraph. 
There are also some open problems in the area of graph similarity. As we mentioned in Section 3.2, we have no concept of the opposite (inverse, negative, etc.) of a graph. Thus we have no definition of what it means to have 0 similarity between two graphs. A second area in need of improvement is the time complexity of some of the algorithms. These methods rely on solving NP-complete problems or have large potential for combinatorial explosion. Approximate and sub-optimal solutions have been proposed, however these are not guaranteed to reach the optimal solution and may require management of various extra parameters. A third problem: some of the approaches described in this chapter are applicable to determining similarity between two graphs at a time only. This is a problem when trying to match a large database of graphs to a single input graph. Messmer and Bunke [86] propose a method of decomposing large graphs into smaller components and then organizing these components into a network which indicates the relationship between the parts in order to deal with this issue. Finally, there have been no extensive cross-comparison experiments performed between these different methods. Most experimental results are in the area of stability analysis or in comparing performance within a certain framework for certain problem areas (e.g. within the state space search approach).

In the following chapters, we will be most interested in the minimum common subgraph approaches, as we have discovered methods of overcoming this method's shortcomings while retaining its strengths. We will also make use of the graph median. In the next chapter we describe how web document content can be modeled with graphs. 


\section{Chapter Four}

\section{Graph Models for Web Documents}

In the previous chapter we reviewed various graph-theoretical techniques for determining graph similarity. Of particular interest to the work presented here are the distance measures based on the computation of the maximum common subgraph of a pair of graphs (Section 3.4) and the median of a set of graphs (Section 3.9). The distance measures based on maximum common subgraph (Eqs. 3.3-3.7) are straightforward in the sense that no space search or parameter value selection is required, but the drawback is that determining the maximum common subgraph is usually an NP-Complete problem. However, for the proposed graph representations introduced here, the determination of the maximum common subgraph can be achieved in polynomial time, as we will see later in this chapter.

In this chapter we will describe several methods for representing web document content (or text documents in general) as graphs. These methods are named: standard, simple, $n$-distance, $n$-simple distance, absolute frequency and relative frequency. Each method is based on examining the terms on each web page and their adjacency. Terms are extracted by looking for runs of alphabetical characters separated by spaces or other types of common punctuation marks. Once the terms are extracted, we use several steps to reduce the number of terms associated with each page to some representative set. This is done to remove irrelevant terms and to reduce computation time.

\subsection{Pre-Processing}

When creating a graph model of a web document, we do not model the entire document as a graph. In order to reduce memory requirements and reduce computation time, we perform a series of pre-processing steps to arrive at a reduced set of the most important terms. First, we have a file of approximately 600 stop words, such as "the," "and," and "of," that contribute little information which we remove if they are present. We also perform some simple stemming in order to determine those word forms which should be considered to be identical (e.g. "test" and "tests"). Stemming is often used in information retrieval to reduce the size of term vectors by conflating those terms which are considered to be identical after the removal of their suffixes. The most common stemming algorithm is the one created by Porter [102]. Porter's algorithm is a fairly straightforward method that applies simple transformation rules in a series of steps in order to remove all the suffixes from a term, leaving only the "stem." Another approach to stemming besides using rules to remove suffixes is to create a database of words and their relationships. The WordNet system uses such a database [89]. Lovins' algorithm [73] is a mix of both the rule-based and database approaches. In Lovins' method, a list of 
approximately 260 suffixes is specified as a mini-database. Associated with each ending is a condition code that specifies some additional conditions that must be met to allow removal of the suffix. After the suffix is removed, some rules are applied to the remaining word to transform it to its final state.

The method of stemming we use is very simple. For each term, we look for plural forms by adding "-s" or "-es" and check to see if the plural form exists. Similarly for verbs ending in "-ing" we either add "-ing" (if the word does not end in "e") or by removing the trailing in "e" and then adding "-ing." If we determine that terms are alternate forms of each other, we conflate to the most frequently occurring form. This gives us good results; for example, when representing documents about "data mining," we conflate to "mining" and not "mine." After handling these cases, we select the most frequently occurring terms as nodes for the graph representing the document. This is very similar to the method described in [66] for extracting the index terms, except that we perform stemming.

We should also make some mention here of other approaches to reducing the number of terms that come from the information retrieval field. It has been noted before that when using the vector space model of information retrieval, performing calculations on vectors with a large number of elements (terms) can be very time consuming. A popular information retrieval technique for reducing the number of terms used is latent semantic indexing (LSI) [32]. This approach uses singular value decomposition, a statistical dimensionality reduction method, to arrive at a reduced set of terms. LSI can achieve good results, but it is computationally expensive. It is also possible to simply drop terms with low weights (called truncation), as computed by some information retrieval metric, such as term frequency within a document. Experimental results have shown that reduced vectors (either by LSI or truncation) produce clusterings which are as good as using the original vectors, and are much faster to compute [118].

Stop word removal, stemming, and LSI are all related to the topic of information retrieval, or, more specifically, information extraction. The main goal of an information retrieval system is to provide users with relevant documents based on a query. We should emphasize that the methods presented in this dissertation are not information retrieval systems. We do not select documents for retrieval, we are clustering or classifying them through the application of machine learning techniques. As mentioned in Chapter 2, a popular model used in information retrieval is the vector space model [107]. In the vector space model, each document is represented in a Euclidean feature space $\mathfrak{R}^{m}$, where $m$ is the number of terms (words) used as features. Each feature indicates the number of times a term appears in a document, or is a weight computed based on some statistical properties.

\subsection{Graph Representations of Web Documents}

We have several methods of creating graphs from web documents: standard, simple, $n$-distance, $n$-simple distance, absolute frequency and relative frequency. Previously, in Section 3.4, we stated that the usual "size" of a graph, $|G|$, is defined as the number of nodes in the graph. However, for our particular representations of web documents it is detrimental to ignore the contribution of the edges, which indicate the 
number of phrases (term adjacencies) identified in the content. Further, it is possible to have more than one edge between two nodes for certain representations. Thus we will use the following definition of graph size for all representations except the frequency representations (the size of a graph under the frequency representations will be described in detail below). Formally, the size of a graph $G=(V, E, \alpha, \beta)$, denoted $|G|$, is defined as:

$$
|G|=|V|+|E|
$$

Thus we will take the size to be the sum of the number of vertices and edges in the graph.

Under the standard representation method each term after stop word removal and stemming becomes a vertex in the graph representing that document. Each node is labeled with the term it represents. Note that we create only a single vertex for each word even if a word appears more than once in the text. Thus each vertex in the graph represents a unique word and is labeled with a unique term not used to label any other node. Second, if word $a$ immediately precedes word $b$ somewhere in a "section" $s$ of the document, then there is a directed edge from the vertex corresponding to term $a$ to the vertex corresponding to term $b$ with an edge label $s$. We take into account sentence terminating punctuation marks (periods, question marks, and exclamation points) and do not create an edge when these are present between two words. Sections we have defined for HTML documents are: title, which contains the text related to the document's title and any provided keywords (meta-data); link, which is text that appears in clickable hyper-links on the document; and text, which comprises any of the readable text in the document (this includes link text but not title and keyword text). Next, we remove the most infrequently occurring words on each document, leaving at most $m$ nodes per graph ( $m$ being a user provided parameter). This is similar to the dimensionality reduction process for vector representations [107]. Finally, we perform a simple stemming method and conflate terms to the most frequently occurring form by re-labeling nodes and updating edges as needed. An example of this type of graph representation is given in Fig. 4.1. The ovals indicate nodes and their corresponding term labels. The edges are labeled according to title (TI), link (L), or text (TX). The document represented by the example has the title "YAHOO NEWS", a link whose text reads "MORE NEWS", and text containing "REUTERS NEWS SERVICE REPORTS". Note there is no restriction on the form of the graph and that cycles are allowed. This novel document representation takes into account additional web-related content information (specifically, the web document section where the terms appear is captured by the edge labels) which is not done in traditional information retrieval models. As we mentioned in Chapter 2, a problem with web content mining systems is that they discard or ignore this web-related information and fall back on the traditional methods that deal only with plain-text documents. Other methods which take web-related information into account require new frameworks for dealing with the additional information, but our method allows us to define different document representations, described below, without changing the basic data mining algorithms. 


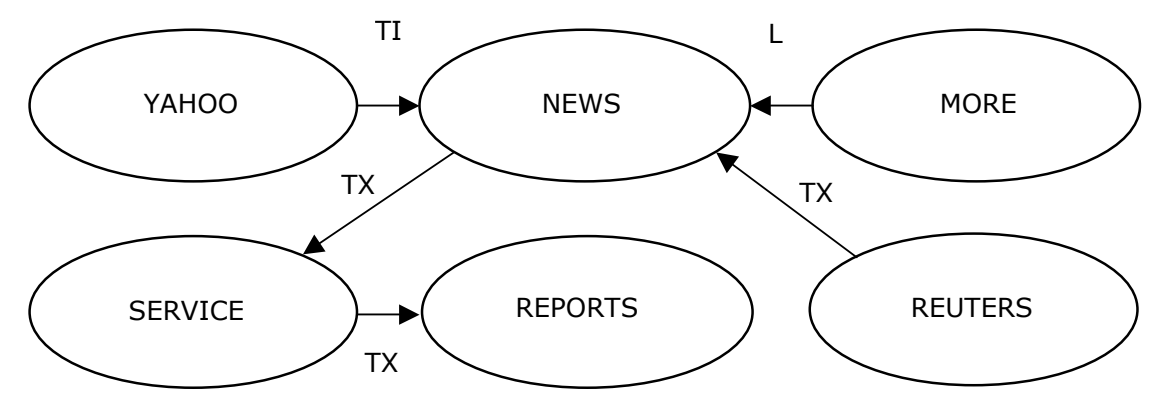

Figure 4.1. Example of a Standard Graph Representation of a Document

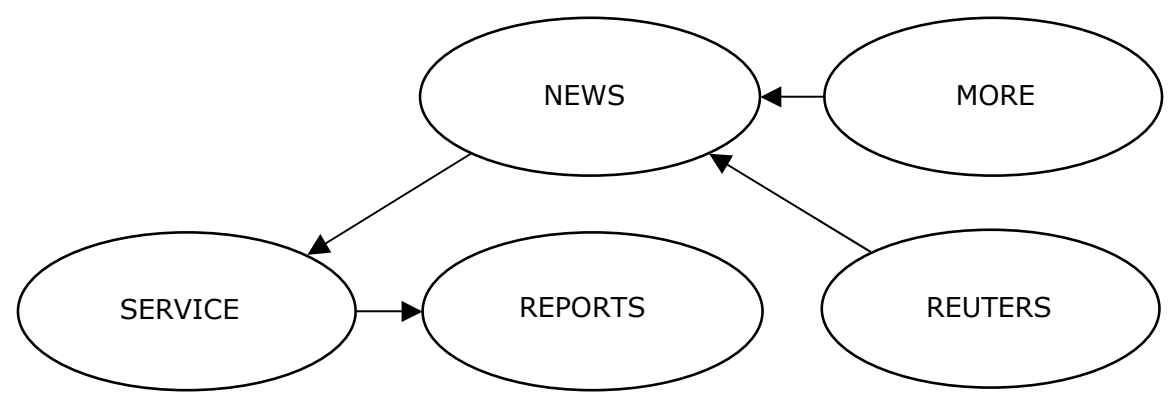

Figure 4.2. Example of a Simple Graph Representation of a Document

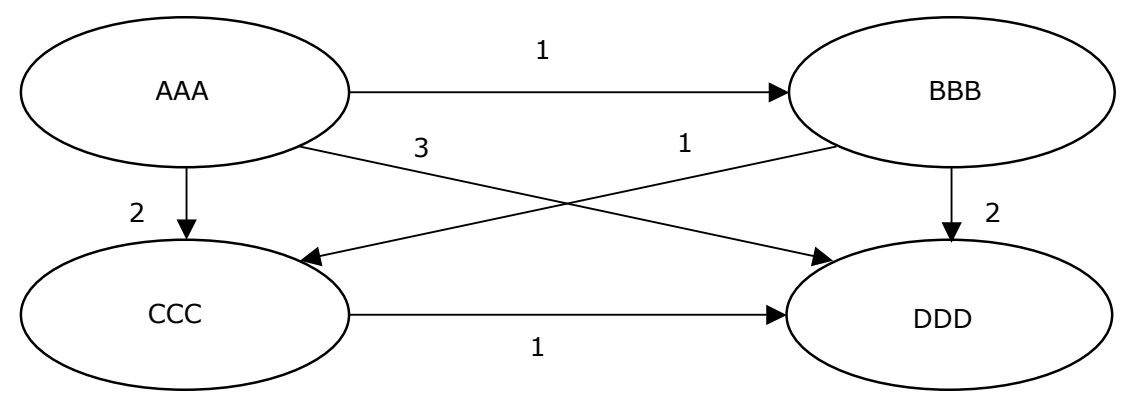

Figure 4.3. Example of an $n$-Distance Graph Representation of a Document

The second type of graph representation we will look at is what we call the simple representation. It is basically the same as the standard representation, except that we look at only the visible text on the page (no title or meta-data is examined) and we do not label the edges between nodes. Thus we ignore the information about the "section" where the two respective words appear together. An example of this type of representation is given in Fig. 4.2.

The third type of representation is called the $n$-distance representation. Under this model, there is a user-provided parameter, $n$. Instead of considering only terms immediately following a given term in a web document, we look up to $n$ terms ahead and connect the succeeding terms with an edge that is labeled with the distance between them (unless the words are separated by certain punctuation marks). For example, if we had the 
following text on a web page, "AAA BBB CCC DDD", then we would have an edge from term AAA to term $\mathrm{BBB}$ labeled with a 1, an edge from term AAA to term CCC labeled 2, and so on. The complete graph for this example is shown in Fig. 4.3.

Similar to $n$-distance, we also have the fourth graph representation, $n$-simple distance. This is identical to $n$-distance, but the edges are not labeled, which means we only know that the distance between two connected terms is not more than $n$.

The fifth graph representation is what we call the absolute frequency representation. This is similar to the simple representation (adjacent words, no sectionrelated information) but each node and edge is labeled with an additional frequency measure. For nodes this indicates how many times the associated term appeared in the web document; for edges, this indicates the number of times the two connected terms appeared adjacent to each other in the specified order. For this representation we modify our definition of graph size (Eq. 4.1). Under the absolute frequency representation the graph size is defined as the total of the node frequencies added to the total of the edge frequencies. Further, when we compute the maximum common subgraph we take the minimum frequency element (either node or edge) as the value for the mcs. For example, if we had two graphs that each had a node $A$, one with frequency of 10 and the other with frequency of 20, then node $A$ in the $m c s$ would have frequency of 10 .

The final graph representation is the relative frequency representation, which is the same as the absolute frequency representation but with normalized frequency values associated with the nodes and edges. The absolute frequency representation uses the total number of term occurrences (on the nodes) and co-occurrences (edges). A problem with this representation is that large differences in document size can lead to skewed distances, similar to the problem encountered when using Euclidean distance with vector representations. Under the relative frequency representation, instead of associating each node with the total number of times the corresponding term appears in the document, a normalized value in $[0,1]$ is assigned by dividing each node frequency value by the maximum node frequency value that occurs in the graph; a similar procedure is performed for the edges. Thus each node and edge has a value in $[0,1]$ associated with it, which indicates the relative frequency of the term (for nodes) or co-occurrence of terms (for edges).

These forms of knowledge representation are a type of semantic network, where nodes in the graph are objects and labeled edges indicate the relationships between objects [106]. The conceptual graph is a type of semantic network sometimes used in information retrieval [74]. With conceptual graphs, terms or phrases related to documents appear as nodes. The types of relations (edge labels) include synonym, part-whole, antonym, and so forth. Conceptual graphs are used to indicate meaning-oriented relationships between concepts, whereas our method indicates structural relationships that exist between terms (content) in a web document. Our method of document representation is somewhat similar to that of directed acyclic word graphs (DAWGs) [27], which is most commonly used for compact dictionary representation. However, in our representations nodes represent words rather than letters, we allow for cycles and multiple edges between nodes, and the edges in our graphs are labeled. It is important to note that while our representations appear superficially similar to the bigram, trigram, or $N$-gram models [122], those are statistically-oriented approaches based on word 
occurrence probability models. Our method does not require or use the computation of term probabilities.

\subsection{Complexity Analysis}

Calculating the distance between two graphs (Eqs. 3.3-3.7) requires the computation of the maximum common subgraph of the pair of graphs. The determination of the maximum common subgraph in the general case is known to be an NP-Complete problem [88]. (Methods for computing the mcs are presented in [69][83].) However, with our graph representation for documents each node in a graph has a unique label (representing a unique term) that no other node in the graph has. Thus the maximum common subgraph, $G_{\mathrm{mcs}}$, of a pair of graphs, $G_{1}$ and $G_{2}$, can be created by the following procedure:

(1) Find the nodes $V_{\text {mcs }}$ by determining the subset of node labels that the original graphs have in common with each other and create a node for each common label.

(2) Find the edges $E_{\mathrm{mcs}}$ by examining all pairs of nodes from step (1) and introduce edges that connect pairs of nodes in both of the original graphs.

We see that the complexity of this method is $O\left(\left|V_{1}\right| \cdot\left|V_{2}\right|\right)$ for step (1), since we need only compare each node label from one graph to each node label of the other and determine whether there is a match or not. Thus the maximum number of comparisons is $\left|V_{1}\right| \cdot\left|V_{2}\right|$, and since each node has a unique label we only need to consider each combination once. The complexity is $O\left(\left|V_{\mathrm{mcs}}\right|^{2}\right)$ for step (2), since we have $\left|V_{\mathrm{mcs}}\right|$ nodes and we look at all combinations of pairs of nodes to determine if an edge should be added between them or not:

$$
\left(\begin{array}{c}
\left|V_{m c s}\right| \\
2
\end{array}\right)=\frac{\left|V_{m c s}\right| !}{\left(\left|V_{m c s}\right|-2\right) ! \cdot 2 !}=\frac{\left|V_{m c s}\right| \cdot\left(\left|V_{m c s}\right|-1\right)}{2}<\left|V_{m c s}\right|^{2}
$$

Thus the overall complexity is $O\left(\left|V_{1}\right| \cdot\left|V_{2}\right|+\left|V_{\text {mcs }}\right|^{2}\right) \leq O\left(|V|^{2}+\left|V_{\text {mcs }}\right|^{2}\right)=O\left(|V|^{2}\right)$ if we substitute $V=\max \left(\left|V_{1}\right|,\left|V_{2}\right|\right)$. 


\section{Chapter Five}

\section{The Graph Hierarchy Construction Algorithm for Organizing Web Search Results}

\section{$5.1 \quad$ Introduction}

In this chapter we will describe our Cluster Hierarchy Construction Algorithm (CHCA) and Graph Hierarchy Construction Algorithm (GHCA): novel hierarchical clustering algorithms which we have implemented in a system that clusters search results obtained from conventional web search engines. We gave our original descriptions of this system in [110][111]. The basic operation of our search clustering system is to take a user provided search string, pass it on to a web search engine (or group of engines), examine the results from those engines, then finally display to the user a group of hierarchically arranged clusters (topics) related to the search subject. Clusters are displayed by printing the list of terms associated with the cluster, which are derived from either the vector (CHCA) or graph (GHCA) representing the cluster. Web pages found by the search engines are assigned to these clusters. The major benefit of our web content mining system over conventional search engines is the differentiation of results based on the related topics and sub-topics. Conventional search engines usually return a large ranked list that is not organized according to topic. Thus users of our system can focus on only the specific areas they are interested in when viewing the results, potentially increasing the speed with which the desired web pages are found. A second benefit is that the collection of topics and their organization provides the user with additional knowledge about the search query topic and how it is related to similar topics. Other benefits of our system include the ability to perform analysis on the full text of the actual documents, when operated in asynchronous (off-line) mode.

There are a number of different algorithms for performing clustering, including hierarchical clustering algorithms which create clusters arranged as a hierarchy rather than partitions. The most well known of these that is widely used for information retrieval is the agglomerative hierarchical clustering method [107]. We will give some details about this algorithm and compare it to our method later in the chapter. An overview of many clustering algorithms and their related issues is given in [58]. More recent surveys of clustering can be found in [59][90]. However, unlike other clustering methods, which do not label clusters according to topic, the interesting features of applying GHCA is not so much the separation of documents into clusters, but rather the cluster labels and the knowledge representation [76][106] induced by the hierarchy of topics.

This chapter is organized as follows. In Section 5.2 we give the Cluster Hierarchy Construction Algorithm, and early vector-based version of GHCA, and describe how it works in detail. In Section 5.3, we describe the relevant implementation details of the 
search result processing system we created. In Section 5.4 we give results of using our system for different query topics as well as comparisons with other similar systems. The GHCA algorithm, a version of CHCA that works with web documents represented by graphs rather than vectors, is given in Section 5.5. Some final remarks are presented in Section 5.6.

\subsection{Cluster Hierarchy Construction Algorithm (CHCA)}

In this section we give the details of the Cluster Hierarchy Construction Algorithm (CHCA), an early form of GHCA that was vector-based, and compare it to similar existing clustering algorithms. We also give a classification of this clustering method based on its characteristics. First we give a review of the concept of inheritance, which is the mechanism used by CHCA and GHCA to create the parent/child cluster relationship.

\subsubsection{A Review of Inheritance}

Inheritance is a technique of creating new, more specialized entities by defining them in terms of existing entities. This terminology and method of creating specialization from existing models comes from the subject object-orientation, which is a software engineering technique that promotes code re-use. In the context of the current work we will apply inheritance to clusters for the purpose of creating new, more specialized clusters that are related to previously existing clusters. Each cluster we create is associated with a topic relating to a web search and is defined by a list of terms that indicate the topic's meaning. When a cluster (called a child cluster or sub-cluster) inherits from another cluster (called a parent cluster or super-cluster) the child receives the terms associated with its parent. Usually, the child cluster will also define new terms of its own. For example, if the parent contains three terms (A, B and C) then its child will have terms $\mathrm{A}, \mathrm{B}$ and $\mathrm{C}$ as well as new terms (e.g. D and $\mathrm{E})$. It is possible for a cluster to have more than one parent, which is called multiple inheritance. Clusters that are not defined using inheritance (i.e. those that have no parents) are called base clusters. Clusters created in this way form a structure called a hierarchy, where children are connected to their parents.

Consider the following example which illustrates the concept. We have a base cluster with a single term: STUDENT. It has two child clusters: UNDERGRADUATE STUDENT and GRADUATE STUDENT. The cluster GRADUATE STUDENT in turn has children MASTERS GRADUATE STUDENT and PH.D. GRADUATE STUDENT. The cluster MASTERS GRADUATE STUDENT is a specialization (i.e. more specific form) of the cluster GRADUATE STUDENT, which in turn is a specialization of the cluster STUDENT. Similarly, STUDENT is a generalization (i.e. less specific form) of the cluster UNDERGRADUATE STUDENT. As we traverse downward through the hierarchy, the clusters become more specific (specialized) and as we move upward they become more general. Observe that in many natural languages, such as English, more specificity is created when more terms are used to describe an entity. Similar to the example above, we see that, for example, "master's degree seeking full-time student" is 
much more specific than simply "student" when describing a person. We make use of this observation in our search clustering system, as clusters with more terms associated with them tend to be specializations of more general clusters.

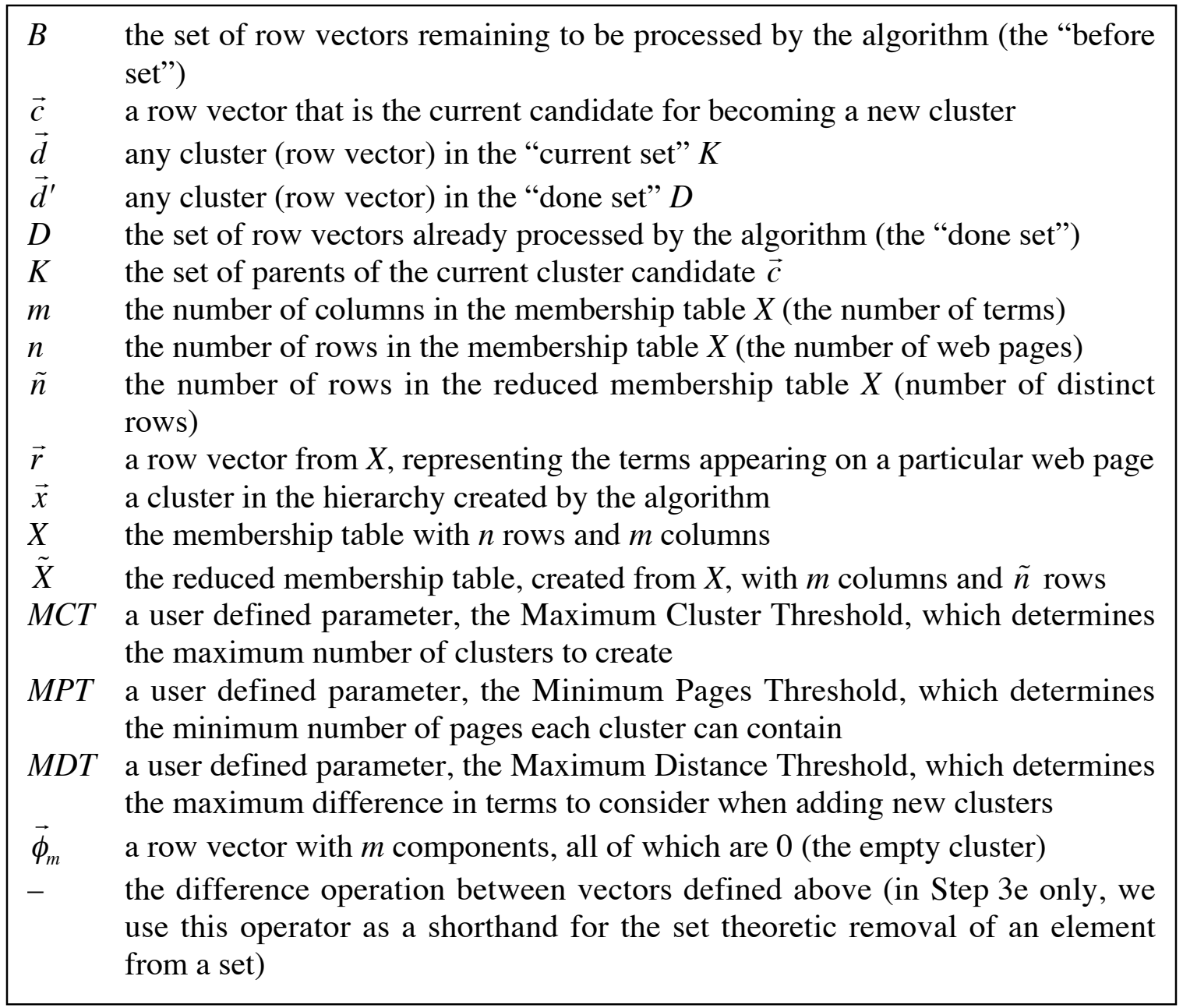

\section{Figure 5.1. Summary of Notation Used in CHCA}

\subsubsection{Brief Overview of $\mathrm{CHCA}$}

Before we describe our algorithm in detail, we present in this sub-section a brief overview of how CHCA works. The algorithm takes a binary matrix (a table) as input. The rows of the table correspond to the objects we are clustering. In this case we are dealing with web pages, but the method is applicable to other domains as well. The columns correspond to the possible attributes that the objects may have (terms appearing on the web pages for this particular application). When row $i$ has a value of 1 at column $j$, it means that the web page corresponding to $i$ contains term $j$. From this table, which is a binary representation of the presence or absence of terms for each web page, we create a reduced table containing only rows with unique attribute patterns (i.e., duplicate rows are removed). Using the reduced table, we create a cluster hierarchy by examining each row, 
starting with those with the fewest terms (fewest number of 1's); these will become the most general clusters in our hierarchy. The row becomes a new cluster in the hierarchy, and we determine where in the hierarchy the cluster belongs by checking if any of the clusters we have created so far could be parents of the new cluster. Potential parents of a cluster are those clusters which contain a subset of the terms of the child cluster. This comes from the notion of inheritance discussed above. If a cluster has no parent clusters, it becomes a base cluster. If it does have a parent or parents, it becomes a child cluster of those clusters which have the most terms in common with it. This process is repeated until all the rows in the reduced table have been examined or we create a user specified maximum number of clusters, at which point the initial cluster hierarchy has been created. The next step in the algorithm is to assign the web pages to clusters in the hierarchy. In general there will be some similarity comparison between the terms of each web page (rows in the original table) and the terms associated with each cluster, to determine which cluster is most suitable for each web page. Once this has been accomplished, the web pages are clustered hierarchically. In the final step we remove any clusters with a number of web pages assigned to them that is below a user defined threshold and re-assign the web pages from those deleted clusters.

\subsubsection{CHCA in Detail}

We will now introduce the formal notation that will be used in the detailed description of the CHCA algorithm as well as the algorithm itself. Let $\vec{a}$ be a bit vector, which is defined as a vector whose components have two possible values: 0 or 1 . In other words, a binary vector. $|\vec{a}|$ is defined as the number of 1 bits in the vector $\vec{a}$, i.e. the Hamming Weight. Let $\vec{a}_{i}$ and $\vec{a}_{j}$ be $x$-bit vectors, i.e. bit vectors with $x$ dimensions or components. Let $\vec{a}_{i} \bullet \vec{a}_{j}$ be the bitwise (component by component) ANDing of two $x$-bit vectors, resulting in a new $x$-bit vector. $\vec{a}_{i}-\vec{a}_{j}$ is defined as abs $\left(\left|\vec{a}_{i}\right|-\left|\vec{a}_{j}\right|\right)$, where abs $(\ldots)$

is the standard absolute value operation. Let $\vec{\phi}_{x}$ be the bit vector of length $x$ with all 0 entries, which is called the empty cluster. Let $\times$ be the standard scalar multiplication operator. Below we present the CHCA algorithm (a summary of notations and variables used in the algorithm is given in Fig. 5.1):

Step 1. Given $n$ sets representing $n$ entities (in the context of the current work, web pages returned by a search engine) each with attributes from a common set with $m$ elements (the common set of terms appearing on all the pages) we create a binary membership table $X$ with $n$ rows and $m$ columns. The entries in each cell of the table will be either 1 or 0 . If the element $j$ from the common set is a member of set $i$, then $X_{i j}=1$; otherwise $X_{i j}=0$. For convenience, each row of the table can be interpreted as an $m$-bit vector that we call a "row vector," denoted by $\vec{r}$.

In this first step we are basically creating a data structure (the membership table $X$ ), which will help us describe and perform the algorithm. The table is a binary representation of the relationships between each web page and each term and is the primary input to the algorithm. The table will have a number of rows corresponding to the number of web pages and a number of columns equal to the total number of terms 
which appear on at least one page. If page $i$ contains the $j$ th term, then at row $i$ and column $j$ in the membership table (denoted $X_{i j}$ ) there will be a 1 ; otherwise there will be a 0 . In the vector space model of information retrieval, this table is called a document-term matrix or an attribute matrix [48][84]. We use the term "membership table" to clarify that this table is used for clustering and not for an information retrieval task.

Step 2. Construct a reduced membership table $\tilde{X}$ by removing duplicate row vectors, leaving only $\tilde{n}(\leq n)$ distinct row vectors. This new data structure $\tilde{X}$ will be used in the next step of the algorithm which actually creates the hierarchy. Since we only consider each unique row vector, we only need the smaller $\tilde{X}$ table to create the hierarchy. This step can be viewed as a pre-processing step which reduces the memory requirements and running time of the main part of the algorithm.

Step 3. Create the cluster hierarchy from $\tilde{X}$ using user provided values for parameters $M C T, M D T$, and $M P T$ (which we will describe in detail after the presentation of the algorithm). The description for the procedure is as follows:

Let $B$, the "before set," be the set of row vectors not yet assigned to any cluster. It initially contains all the row vectors of $\tilde{X}$, namely $\left\{\vec{r}_{1}, \ldots, \vec{r}_{\tilde{n}}\right\}$. Set $D$, the "done set," is the set of clusters created by the algorithm so far. $D$ is initially set to $\left\{\vec{\phi}_{m}\right\}$. While $B \neq \varnothing$ (i.e. non-empty, there are still candidates to process) and $|D| \leq M C T+1$ do the following steps:

(Step 3a) Let $\vec{c}$ be a row vector from set $B$ such that for all $\vec{a}_{i} \in B,|\vec{c}| \leq\left|\vec{a}_{i}\right|$. In other words, $\vec{c}$, the "candidate vector," is a row vector from set $B$ which has a minimum number of terms ( 1 bits). If there is more than one row with the same minimum number of terms, select one at random for $\vec{c}$.

(Step 3b) Find a set of clusters $K \subseteq D$ such that $\vec{c}-\vec{d} \leq \vec{c}-\vec{d}^{\prime}$ and $\vec{c} \cdot \vec{d}=\vec{d}$ for all $\vec{d} \in K$ and $\vec{d}^{\prime} \in D$. That is, determine the subset $K$ of cluster(s) in $D$ (i.e. in the existing cluster hierarchy) that are parent(s) of the candidate row vector $\vec{c}$. The parent clusters in set $K$ must satisfy the following two conditions simultaneously:

(1) Subsethood. The terms of the row vector(s) in $K$ are subsets of the terms of row $\vec{c} \cdot \vec{c} \cdot \vec{d}=\vec{d}$ states that $K$ only consists of those clusters such that the attributes of the parent clusters (those in $K$ ) are a subset of those of the child cluster. This is in-line with the notion of inheritance: the child cluster has all the terms of its parents, plus some newly defined terms. Thus a parent cluster's terms are a subset of those of its children.

(2) Minimum Distance. When using the vector difference operation we defined above, i.e. abs $(|\vec{c}|-|\vec{d}|)$, with vectors that satisfy the subsethood condition just described, the operation gives the number of terms that differ between parent and 
child clusters; the number of common terms is given by $|\vec{c}|$. The condition $\vec{c}-\vec{d} \leq \vec{c}-\vec{d}^{\prime}$ means the direct parents of $\vec{c}$ are those clusters that have the least "distance" (difference in terms) from the child (or, put another way, the parents of $\vec{c}$ are those clusters with the most terms in common). This is needed to enforce a proper ordering among the clusters. For example, consider the following hierarchy that should nominally be created: cluster $a$ is the parent of cluster $b$ and cluster $b$ is the parent of cluster $c$. If we do not enforce some kind of ordering, we could instead have both cluster $b$ and $c$ as a direct children of $a$, since by transitivity cluster $a$ 's attributes are a subset of both $b$ 's and $c$ 's. Further, the empty cluster $\vec{\phi}_{m}$ is by definition a subset (parent) of every cluster. Without this second condition we could end up with all clusters inheriting from the empty cluster and becoming base clusters. Using the minimum distance condition we attach child clusters only to their most similar parent(s).

(Step 3c) If the minimum of $\vec{c}-\vec{d}$ for all $\vec{d} \in K \leq M D T$ or $K$ contains $\vec{\phi}_{m}$ (i.e. $\vec{c}$ is a base cluster), then candidate vector $\vec{c}$ becomes the child of all clusters in $K$ by inheritance (or multiple inheritance if $K$ has more than one element). Otherwise, skip to Step 3e. Clusters with $\vec{\phi}_{m}$ (the empty cluster) as a parent are base clusters. Each child cluster inherits the terms from its parent cluster(s) and adds its new terms.

(Step 3d) $D=D \cup \vec{c}$. In other words, add the row vector corresponding to $\vec{c}$ to the set $D$ (the "done set").

(Step 3e) $B=B-\vec{c}$. That is, remove the row vector corresponding to $\vec{c}$ from the set $B$ (the "before set").

Step 4. After the loop in Step 3 ends, the initial cluster hierarchy has been created. However the web pages have not yet been assigned to clusters. For each web page (row) in the original membership table $X$, assign the web page to a cluster using the distance measure:

$$
\operatorname{Dis}(r, i)=\frac{1}{m} \sum_{j=1}^{m} Y_{i j} \cdot\left(Y_{i j}-X_{r j}\right)
$$

where $m$ is the number of terms (number of columns), $X_{r j}$ is the $j$ th term in row $r$ of the original membership table, and $Y_{i j}$ is the $j$ th term of cluster $i$ in the hierarchy (the vector corresponding to the cluster). In other words, the distance is the average number of terms in the cluster $i$ that are missing in the web page $r$ (the "extra" terms are ignored). If all the cluster terms appear in the page, the distance is zero. If the page contains none of the terms in the cluster, the distance attains its maximum value (1.0). Otherwise, it takes some value in $[0,1]$ that is proportional to the compatibility of a web page and a cluster. The distance of any document to the empty cluster is zero. We assign each web page to the cluster with the smallest distance. In case of a tie, we prefer clusters with the most terms (i.e. those that are most specific). If we tie again on that criteria, we choose a 
cluster from among those tied clusters with the fewest number of assigned web pages to try to balance out the number of pages per cluster.

Step 5. Starting with the clusters farthest down in the hierarchy, remove those clusters that have a number of web pages assigned to them less than MPT. Reassign the pages from the deleted clusters to the remaining clusters. Repeat this process until no cluster has fewer than $M P T$ pages. Give children of deleted parents updated parents using the subsethood and minimum distance criteria given in Step $3 b$ above.

The CHCA algorithm includes 3 user specified parameters to control the results. The first parameter is a threshold for the maximum number of clusters to create (MCT, maximum cluster threshold). Without this constraint, CHCA will create a cluster for each row in the reduced membership table. However, this can be a large number of clusters, especially if the number of columns is large. So for clarity in interpreting and viewing the cluster hierarchy we limit the number of clusters to be created. Note the check is for $M C T+1$ in Step 3 due to the fact that $D$ contains the empty cluster in addition to the clusters created and thus its cardinality is always 1 greater than the actual number of clusters created so far. Another parameter is a maximum distance threshold between parent and child clusters (MDT, maximum distance threshold). When a new cluster is added to the hierarchy, it adds a certain number of new terms to its parent's terms (even if the cluster is a base cluster and its parent is the empty cluster). Depending on the application, we may wish to avoid adding those clusters which add too many new terms in one step (they overspecialize). For example, consider a cluster with 2 terms: A, B. There are two possible child clusters of this cluster: one with terms $\mathrm{A}, \mathrm{B}, \mathrm{C}$ and one with terms A, B, D, E, G, H, K. The distance between the former and its parent is 1 term. The distance between the latter and its parent is 5 terms. By changing this parameter we can control the size of the "jumps" we wish to allow (base clusters are exempt from the check). The final parameter is the minimum allowed pages per cluster (MPT, minimum pages threshold). It may be that some clusters are initially assigned only a few web pages. The minimum pages threshold allows us to specify the minimum number of pages a cluster should have. If after creating the hierarchy there are clusters that are assigned less than this number of pages, we delete those clusters, starting with the clusters at the lowest levels of the hierarchy. As we delete each cluster that has less than MPT pages, we reassign the deleted cluster's web pages using the same method just described in Step 4. After all the clusters with a number of pages below MPT are deleted, we update the hierarchy structure by giving parents to orphaned clusters whose original parents were deleted, using the same method as in Step $3 b$.

Each cluster has associated with it a bit vector, which indicates its related terms. When we display the topic (label) of each cluster in the hierarchy to the user, we print out the list of corresponding terms which have a 1 bit for that cluster's vector; $e . g$. if cluster 5 has a 1 bit at columns 17 and 24, we print out the terms associated with columns 17 and 24 when displaying cluster 5 . The terms are printed in alphabetical order, since no ordering information is preserved in the vector representation (i.e. we do not know which term comes first on a given web page). Later, we will show how GHCA uses graphs to preserve the ordering information. 
We will now discuss the computational complexity of the algorithm. $n$ is the number of rows in the original membership table (the number of web pages) and $m$ is the number of columns (the total number of terms). Note that the worst case occurs when the reduced membership table and the original table are identical in size $(n=\tilde{n})$. We start with Step 2, as Step 1 is basically a description of how to prepare the input to CHCA. For Step 2, the complexity is $O\left(n^{2} m\right)$. This is due to the fact that we need to compare each of the $n$ vectors with the others, and each comparison consists of checking $m$ bits of the vector. In Step 3a, the complexity of a single iteration is $O(\mathrm{~nm})$, since we check $m$ bits each in at most $n$ vectors in the worst case. For Step $3 \mathrm{~b}$, it is $O(\mathrm{~nm})$ for a single iteration. Steps 3c, 3d, and 3e are each $O(1)$. Step 3 is executed $n$ times in the worst case (when $M C T \geq n)$, thus the overall complexity for Step 3 in $O\left(n^{2} m\right)$. For Step 4, we have $O\left(n^{2} m\right)$ for the worst case, since the distance measure will make comparisons for all $m$ vector components. For Step 5, we have a maximum of $n$ checks or deletions of clusters (since the maximum number of clusters in the worst case is $n$ ). Each deletion requires the reassignment of at most $O(M P T)=O(1)$ web pages, which in turn requires $O(\mathrm{~nm})$ computations. So the overall complexity for Step 5 is $O\left(n^{2} m\right)$. Thus the time complexity for CHCA is $O\left(n^{2} m\right)$ in the worst case.

Table 5.1. Simple Example to Illustrate Concepts of CHCA

\begin{tabular}{|c|c|c|c|c|}
\cline { 2 - 5 } \multicolumn{1}{c|}{} & $\begin{array}{c}\text { Has High } \\
\text { Performance } \\
\text { Engine }\end{array}$ & $\begin{array}{c}\text { Works on } \\
\text { Land }\end{array}$ & Can Fly & Floats \\
\hline BOAT & 0 & 0 & 0 & 1 \\
\hline SPEED BOAT & 1 & 0 & 0 & 1 \\
\hline CAR & 0 & 1 & 0 & 0 \\
\hline RACE CAR & 1 & 1 & 0 & 0 \\
\hline
\end{tabular}

\subsubsection{CHCA: an Example}

A simple example is in order to clarify the ideas and terminology of CHCA that we introduced above. The following is just a general example of creating the hierarchy and is not necessarily related to processing web search results. For brevity we will omit the steps which deal with assigning web pages to clusters (Step 4 and Step 5); this is an example of creating a cluster hierarchy only. For this example, let us consider the domain of vehicles. The membership table $X$ for this example is given in Table 5.1. Here we have 4 entities ( $n=4$ rows): BOAT, SPEED BOAT, CAR, and RACE CAR. We also have 4 possible attributes that each entity could have, and thus $m=4$ columns. In this case, each row is unique and thus there is no reduced membership table (it is already reduced). Assume $M C T=4$ and $M D T=4$.

We initialize $B$ as the set of row vectors of $\tilde{X}, B=\{[0001],[1001],[0100],[1100]\}$ and set $D=\left\{\vec{\phi}_{4}\right\}=\{[0000]\}$. Next we select the row vector from $B$ with the minimum Hamming Weight, which is either BOAT [0001] or CAR [0100] since both have a weight of 1 , to be the new candidate vector $\vec{c}$. Since there is a tie, we arbitrarily select BOAT, so $\vec{c}=[0001]$. After that we determine which clusters in $D$ can be parents of $\vec{c}$. So far there is only element in $D$, the empty cluster [0000]. Thus BOAT becomes a base cluster since 
the empty cluster is its parent, and we update $B$ and $D$ by adding $\vec{c}$ to $D$ and removing it from $B$. Note that we skip the distance $(M D T)$ check since $\vec{c}$ is a base cluster (i.e. $K$ contains $\left.\vec{\phi}_{4}\right) . B$ is now $\{[1001],[0100],[1100]\}$ and $D$ is updated to $\{[0000],[0001]\}$. We repeat the process since $B$ is not empty and $|D|=2<M C T+1$. We will select CAR [0100] since it has minimum weight. It will also be a base cluster and now $B=\{[1001],[1100]\}$ and $D=\{[0000],[0001],[0100]\}$. Another iteration through the loop, we can select either SPEED BOAT or RACE CAR from $B$ since their weights are equal at 2. We choose SPEED BOAT. This time, $K$ will be $\{[0001]\}$ as [0001] is the subset of [1001] that has minimum distance: [1001]-[0001]=1 whereas [1001]-[0000]=2. Thus the cluster SPEED BOAT becomes the child of the cluster BOAT, since the distance (1) is less than $M D T$ (4). The algorithm performs one more iteration and the resulting cluster hierarchy is shown in Fig. 5.2. The results seem intuitive: a hierarchy where we move from the general cases to the specific as we travel downwards has been created.

In Section 5.3, we will describe some important implementation details of our web search processing system. In order to get good results in our application we have added another parameter related to pre-processing the input to CHCA. We will discuss this parameter as well as the values we selected for $M C T, M D T$, and $M P T$ for our application in the next section.
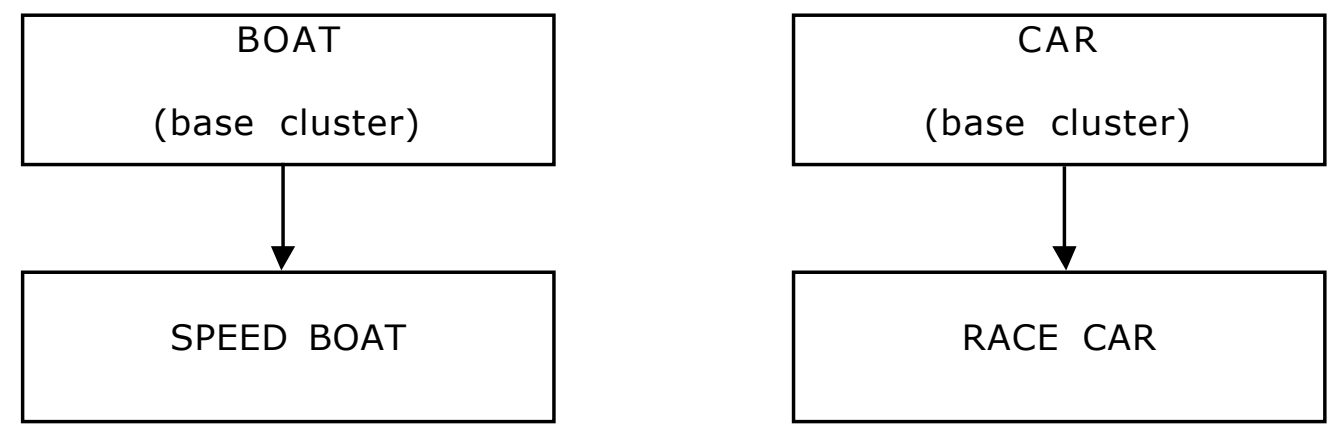

Figure 5.2. Cluster Hierarchy Created from the Example in Table 5.1

\subsubsection{Examination of CHCA as a Clustering Method}

In [58][59], Jain et al. give various characteristics which all clustering methods have, in order to differentiate and classify them. In this sub-section we will attempt to classify our clustering algorithm, CHCA, using those criteria. We will also mention clustering algorithms which are similar to CHCA that have been reported in the literature.

The first criteria is whether CHCA is exclusive or non-exclusive. Exclusive means each object belongs to exactly one cluster. Non-exclusive means an object can belong to more than one cluster. As we have described the actual implementation above, CHCA is exclusive. However, it need not be, as the cluster hierarchy creation process is separate from the process that assigns web pages to clusters. We could easily assign a page to more than one cluster, for example, if the distance measure between the page and several clusters were equal. In fact, the graph-based extension of CHCA, GHCA, which we 
introduce later is non-exclusive. The best we can say on the subject is that it is implementation specific: CHCA is neither specifically exclusive or non-exclusive.

The next issue is whether our method is intrinsic or extrinsic. Intrinsic means the clustering method is unsupervised, whereas extrinsic implies the method learns from prepared examples. CHCA is clearly an intrinsic method as we do not provide labeled training examples. The algorithm clusters only on the basis of the data provided. Indeed, with the Internet comprising nearly every subject imaginable it is intractable to create training sets for them all.

Another important issue is whether CHCA is a partitional method, or whether it is hierarchical. Clearly, our method is hierarchical since it creates a cluster hierarchy like other hierarchical clustering methods. We can compare our method to the well known agglomerative hierarchical clustering technique that is popular in the information retrieval literature [48][58][59][90][107]. This method creates a hierarchy of clusters by starting with a separate cluster for each document and then merging the most similar clusters to create larger clusters, which in turn become the parents of the merged clusters. The process is repeated until a tree is created with a single cluster encompassing all the document at the root. Although our method can produce a hierarchy with different characteristics than the agglomerative method (e.g. we allow for multiple root nodes and for multiple inheritance), the graphical interpretation of the resulting hierarchy structure is the same as in the agglomerative hierarchical method. Nodes in the hierarchy are either clusters or the objects that are being clustered (web pages). Web pages appear only as leaf nodes, while the internal nodes are clusters. Links indicate a parent/child relationship (in the case both nodes are clusters) or a "belongs to" relationship (where one node is a web page). In our method we allow a child cluster to have more than one parent, unlike the agglomerative method where every child cluster has exactly one parent. Similarly, if we allow documents to be linked to more than one cluster we arrive at the non-exclusive version of the algorithm. There are also divisive hierarchical clustering algorithms. In those approaches, all objects start out in a single cluster. The idea is to split large clusters into smaller clusters, forming a hierarchy. However, in the literature the agglomerative method is most prevalent.

CHCA cannot be classified as either agglomerative or divisive, since we neither split or join clusters. Instead we should consider CHCA as an iterative method, as it constructs the hierarchy by adding new clusters one at a time (see [46]). It is important to note the difference between conventional hierarchical clustering methods and CHCA in terms of the information provided by the hierarchy that is created. With conventionally created hierarchies, a measure of similarity (such as a distance measure) is used to compare the objects in clusters in order to determine when clusters should be merged into a parent or split into children. This leads to a hierarchy whose characteristic is differentiation between siblings (children of the same cluster). The information imparted by such a hierarchy is the series of nested partitionings which indicate the clustering of the objects and their nesting order. In comparison, CHCA uses the idea of inheritance to create the parent/child relationship. A child cluster must contain all the attributes of its parent. This is different from the notion of similarity used in the conventionally created hierarchies. Thus, the characteristic of a cluster hierarchy created by CHCA is that child clusters are specializations of their parents. In other words, a child cluster contains all the 
attributes of its parent in addition to other attributes which distinguish it from its parents and siblings. Conversely, parent clusters are generalizations of their children: they contain a subset of the child's essential attributes. The information conveyed by an hierarchy created by CHCA includes which clusters are specializations or generalizations of others (the parent/child relationship), which clusters share a common set of elements but also have other differences (the sibling relationship), which terms are most general (those shared between parents and children), and which terms are used to differentiate and specialize the clusters (those terms found in children but not parents).

A similar method of determining parents through subsets of terms is also used in the MSEEC system [51]. One of the main differences between that method and CHCA is that we allow for multiple inheritance (multiple parents) whereas MSEEC uses trees (single parents). Thus MSEEC must break ties for multiple valid parents, which it does by selecting the cluster with the most documents assigned. The other phases of MSEEC, such as controlling the number of clusters and generating their associated terms are quite different from ours (e.g. we eliminate small clusters and re-assign the documents whereas MSEEC merges similar clusters). The STC method used in Carrot ${ }^{2}$ [119] and Grouper [139] also arranges terms in a tree like structure from general (less terms) to specific (more terms). However, STC creates a purely partitional clustering, not a hierarchical one. Collocation networks [79] are another similar method for extracting terms and their relationships from documents; this method uses frequency of term occurrence and mutual information (see Section 6.4) to generate a visualization of a document.

A method similar to CHCA, but which has not been used for web mining, is the hierarchical biclustering HICLAS system described in [31][105]. Like CHCA, HICLAS performs hierarchical clustering on a matrix with binary entries. It also uses the set theoretic notion of subsets to order the clusters, just as CHCA does. But there are several important differences between HICLAS and CHCA. The first difference is that HICLAS performs a sequence of Boolean decompositions on binary matrices in order to create cluster hierarchies. Thus HICLAS is more akin to a direct optimization approach, as it is attempting to optimize the "goodness of fit" of the created hierarchy on the input data [46]. In contrast, CHCA is an iterative approach, as discussed above. A second difference is HICLAS creates two separate cluster hierarchies, one for the objects (rows) and one for the attributes (columns), and then associates clusters in these two hierarchies. CHCA creates a single cluster hierarchy in terms of the attributes (terms) into which objects (web pages) are classified. By assigning web pages to clusters (groups of terms) we are in effect creating a hierarchy incorporating both the terms themselves (the clusters) and the web pages. The hierarchy of terms (i.e. the cluster hierarchy) is created directly in Step 3 of the algorithm, and the hierarchy of web pages is created indirectly by assigning them to clusters in Steps 4 and 5. A third difference is that the subset ordering in our approach is the reverse of the one used in HICLAS. In CHCA, the top level clusters have the fewest associated attributes (i.e. are most general) while those in the lower levels have more; in HICLAS, the top level clusters have the most attributes (i.e. are most specific). CHCA is more in line with the inheritance paradigm, as we discussed above in subsection 5.2.1.

In summary, $\mathrm{CHCA}$ is an intrinsic, iterative, hierarchical clustering method which can be either exclusive or non-exclusive (depending on the implementation) and uses the 
idea of inheritance rather than a traditional measure of similarity to create the cluster hierarchy.

\subsection{Application of CHCA to Search Results Processing}

With the explosion of content on the Internet it is becoming increasingly more difficult for users to find the exact pages containing the information they want to view. Conventional search engines can return hundreds or thousands of pages for a single query. Clearly it is not a good use of the user's time to manually sift through all of the results [62][139]. Our approach to improving this situation is to perform an unsupervised hierarchical clustering of the pages returned by a conventional search engine and organize the topics related to the search in a hierarchy. We note that supervised classification methods are difficult to use for web searches due to the fact that the number of topics (clusters) on the web is very large and highly dynamic and such systems require prior training on a known group of topics. This is one of the reasons we use CHCA for web search clustering, as it is unsupervised and does not require training examples. CHCA has the further benefit that it provides several parameters which allow the user to tailor the characteristics of the hierarchy that is created to a given application. For our web search processing system, we used these parameters to keep the size of the hierarchy and number of pages in each cluster reasonable.

In this section we will describe the important implementation details of our search system.

\subsubsection{Asynchronous Search}

The system that we have created for processing the search results returned by a search engine performs what we call asynchronous search. Unlike most conventional search engines which return a ranked list of documents which match the query within a few seconds, our system works by handling the search and processing off-line. The user submits his query and then is immediately free to go and perform other tasks. Once the search results have been processed the user is notified by e-mail and can view them at his or her leisure. Even though conventional search engines return results quickly, the user is often required to spend time browsing through the results, perhaps having to re-query in order to properly focus the search (e.g. from "Amazon" to "Amazon rain forest"). The main benefit to performing an asynchronous search over the conventional method is that, because users do not have to keep an active connection once a request is submitted, the system can perform some time consuming processing to improve the results since they are not required right away. The user is not kept waiting for a response and he can perform whatever other tasks he wishes while the request is being processed. Thus asynchronous search makes the best use of the user's time: he or she is not kept waiting while the results are processed and he or she is able to work with the results more efficiently when they are available. In addition, the e-mail with a link to the search results can be saved by the user and revisited at a later time; the results are not lost once the user exits the browser or switches to a different computer. 


\subsubsection{Implementation, Input Preparation and Pre-processing}

The basic operation of our system is as follows. First, the user submits a query request to the system via a web form. The request is eventually picked up by our system which in turn queries a standard web search engine (by default we used Google [45]) for web pages matching the user's query. Using the description and title information returned by the search engine or the full text of the web page itself obtained by downloading each URL, we create a list of terms associated with each page. The list of terms associated with each page is used to create the membership table (Step 1 in the CHCA algorithm), which is reduced (Step 2) and passed on to a program that performs the rest of the CHCA algorithm: it creates a cluster hierarchy (Step 3) and assigns each page to a cluster (Step 4). Finally the clusters with small numbers of pages are removed (Step 5). The user is e-mailed with a URL where he can review the results of the algorithm.

By parsing the full text or the description and title of each URL we generate a list of terms which appear on each web page. This is equivalent to creating a row vector in the membership table for each accessible URL. However, the number of columns in the membership table is equal to the number of unique terms encountered over all of the pages. This can be a huge number (thousands), so to both ease memory requirements and speed processing we reduce the number of terms (columns) to a fixed threshold, called MTT (Maximum Term Threshold). The terms that are selected are those that appeared on the largest number of pages. After examining experimental results, we chose a 30 term threshold. This essentially makes the time complexity of CHCA for this implementation $O\left(n^{2}\right)$ since $m$ is now a constant (recall that $n$ is the number of web pages and $m$ is the number of terms). We note here that in our original system we downloaded and parsed the entire HTML of each page. Using only the description and title "snippets" provided by the search engine, the system produces results much more quickly.

\subsubsection{Selection of Parameters for Web Search}

Previously we described the three user defined parameters used in CHCA, MCT, $M D T$, and $M P T$, which are used to change the characteristics of the created hierarchy. However we have not yet given what values we have used for them in our web application. We performed many experiments with these parameters (as well as MTT) in order to determine values which produce reasonable results over a variety of searches. From our experimental results we chose to limit the number of clusters (MCT) at 100. This may seem too high, but it turns out the number of clusters created is small (about 1020 ), due to reasons we will explain in a moment. From experimental results, we chose a distance threshold $(M D T)$ of 2 and chose an $M P T$ of 5 pages per cluster.

By setting the $M C T$ to 100 , we are essentially allowing up to 100 clusters to be created. The distance threshold, $M D T$, further limits the number of potential clusters. A larger minimum page per cluster threshold, $M P T$, causes the hierarchy to be pruned of more clusters, eventually resulting in usually about 10 to 20 reasonably sized clusters for our chosen $M C T$. A plot of the average number of clusters created (taken over the variation of the other parameters for a fixed set of values) as a function of $M C T$ for three separate queries (specified in the legend) that illustrates the relationship is given in 
Fig. 5.3. A similar plot for $M P T$ is given in Fig. 5.4. Note that for these graphs the $M C T$ assumes a maximum value of 40 .

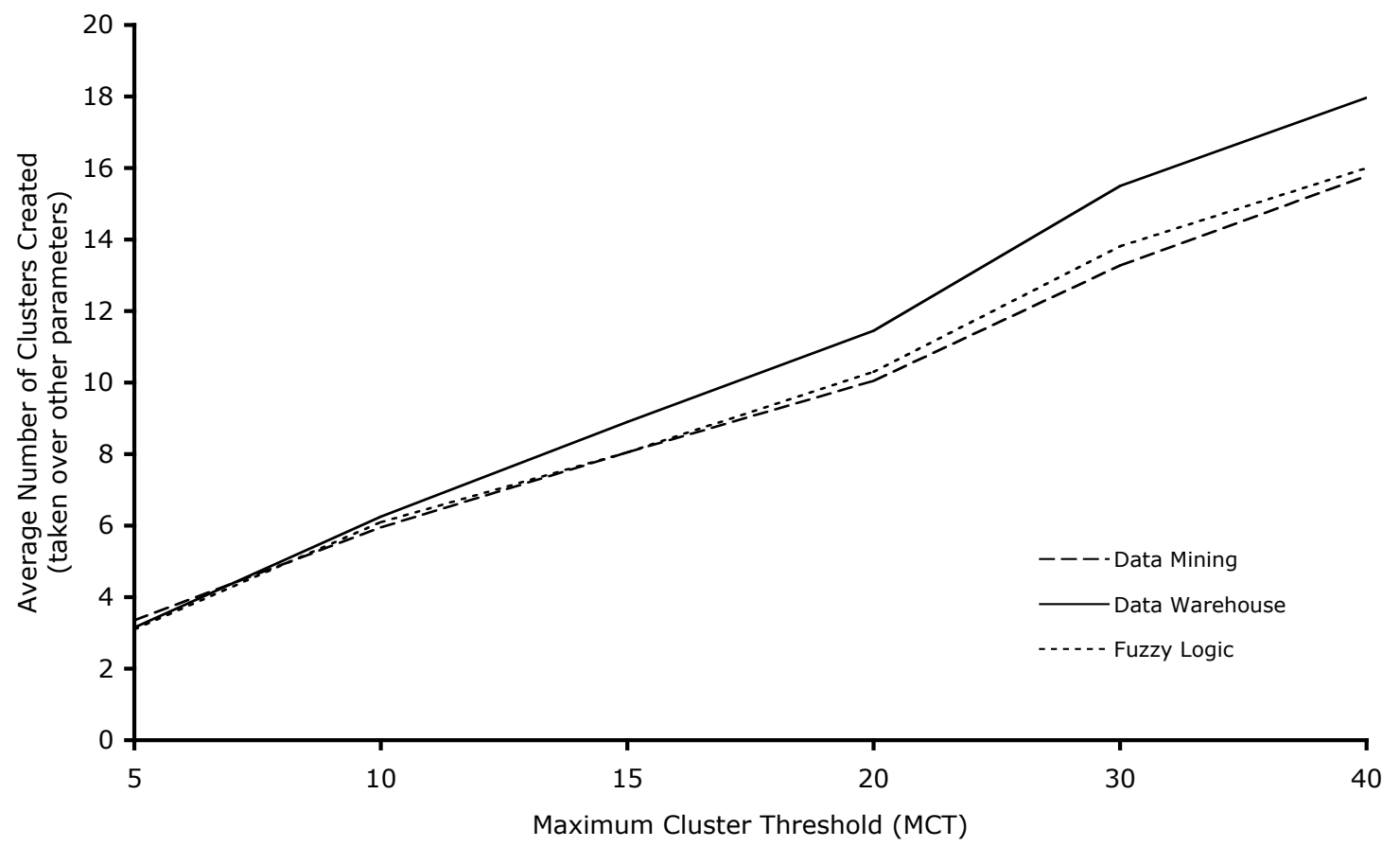

Figure 5.3. Average Number of Clusters Created as a Function of Maximum Cluster Threshold (MCT) for Three Queries

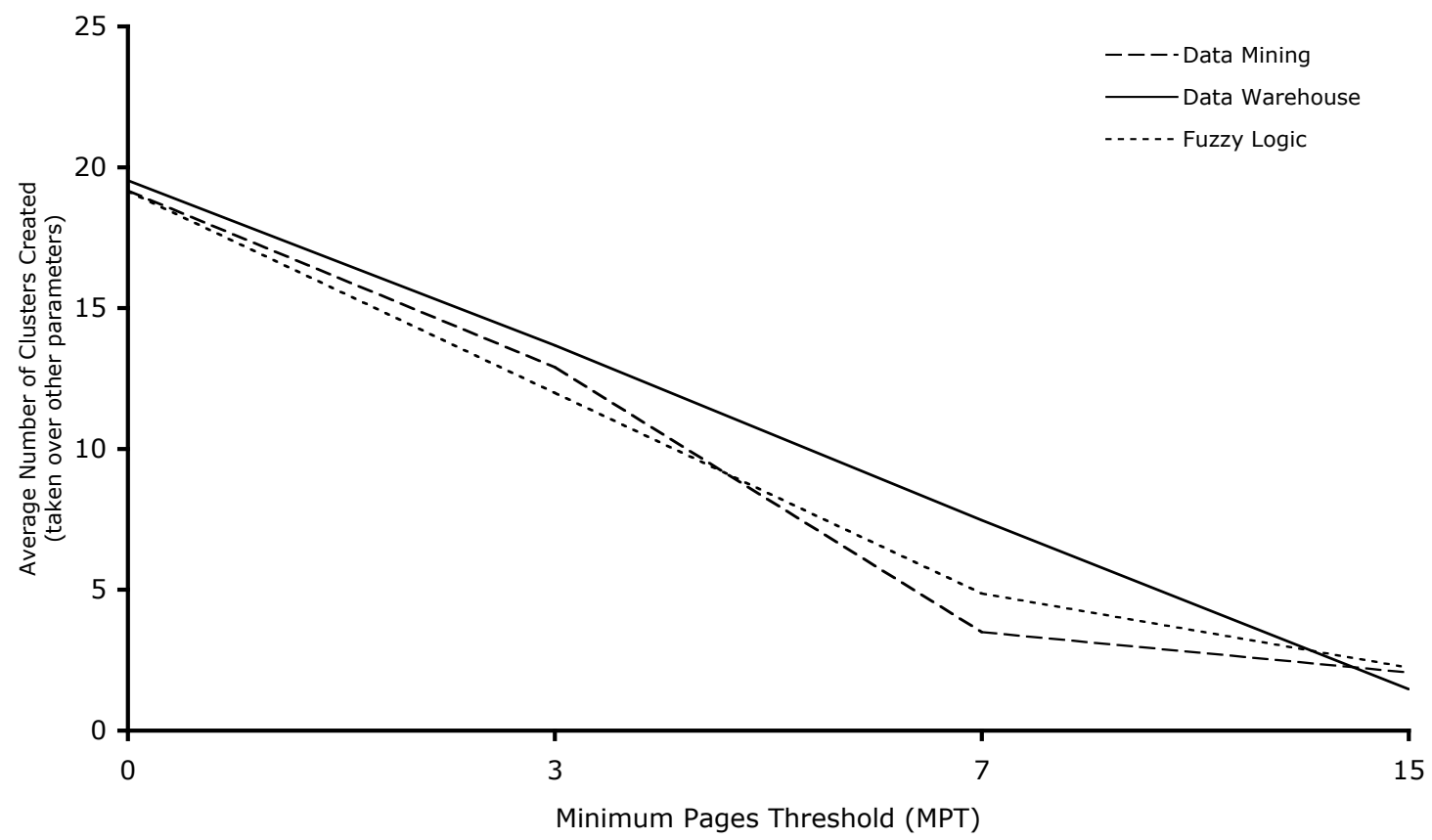

Figure 5.4. Average Number of Clusters Created as a Function of Minimum Pages Threshold (MPT) for Three Queries 
It is worth noting that the parameters, once set to values which produced acceptable hierarchies, do not need to be changed for each query. In our experiments, the choice of search engine(s) has a greater interaction with the parameters than the topic of the query. This is due to the fact that different search engines return varying amounts of results (pages) as well as varying sizes and types of snippets from which to extract the term list of each result.

\section{$5.4 \quad$ Examples of Results}

In this section we present examples of cluster hierarchies produced by our method and compare them with the output of other systems which cluster web search results. Unfortunately, there are some characteristics of CHCA that make it very difficult to compare its output to "ground truth" clustering. The most problematic is the two-phase nature of CHCA. There is a cluster hierarchy creation process and a cluster assignment process. As we will see later, there are methods available for measuring the performance of the latter process (in a partitional sense) but no techniques are available for the former. Much of CHCA's usefulness and novelty comes from the fact that clusters are identified according to topic labels and that the clusters are arranged in a hierarchy. No data sets or performance measures exist that address these issues due to the fact that only a manual comparison by humans using natural language and expert knowledge can suffice. For example, if ground truth has a cluster labeled "WEB BASED DATA MINING" and CHCA creates a cluster "WEB MINING," how "wrong" is this? A second problem is that when performing a cluster comparison, the number of clusters is usually desired to be the same as the number of clusters in ground truth. While CHCA provides a mechanism for limiting the maximum number of clusters, there is no guarantee regarding the actual number of clusters created due to pruning of small clusters, etc. Finally, the fact that multiple inheritance is allowed in CHCA makes it unclear how a partitional clustering would be created by cutting across the hierarchy (as can be done with a dendrogram created from a typical hierarchical clustering method). Due to the above difficulties, we have chosen to evaluate CHCA's performance in our search system by comparing it to two other similar systems.

\subsubsection{Comparison with Grouper}

In Fig. 5.5 we give an actual cluster hierarchy that was constructed by CHCA for the topic "soft computing." Boxes indicate clusters and arrows indicate inheritance from parent to child. The terms listed for each cluster are the terms added by that cluster. Recall that sub-clusters also contain the terms of their parent(s). So, for example, the cluster NEURO in the figure also contains the terms SOFT, COMPUTING, and FUZZY. We omit these inherited terms for clarity. In the actual system the terms associated with each cluster are presented in alphabetical order and not necessarily the order in which they usually appear, but for the figure we have arranged the terms so that they appear in the correct order (e.g. SOFT before COMPUTING). The number of pages assigned to each cluster is also listed at the bottom of each cluster. Note that the clusters created for this topic and the hierarchy itself are quite reasonable. We should mention that the WSC 
term used in one of the clusters is the World Conference on Soft Computing and Engineering Design and Manufacturing, abbreviated to WSC.

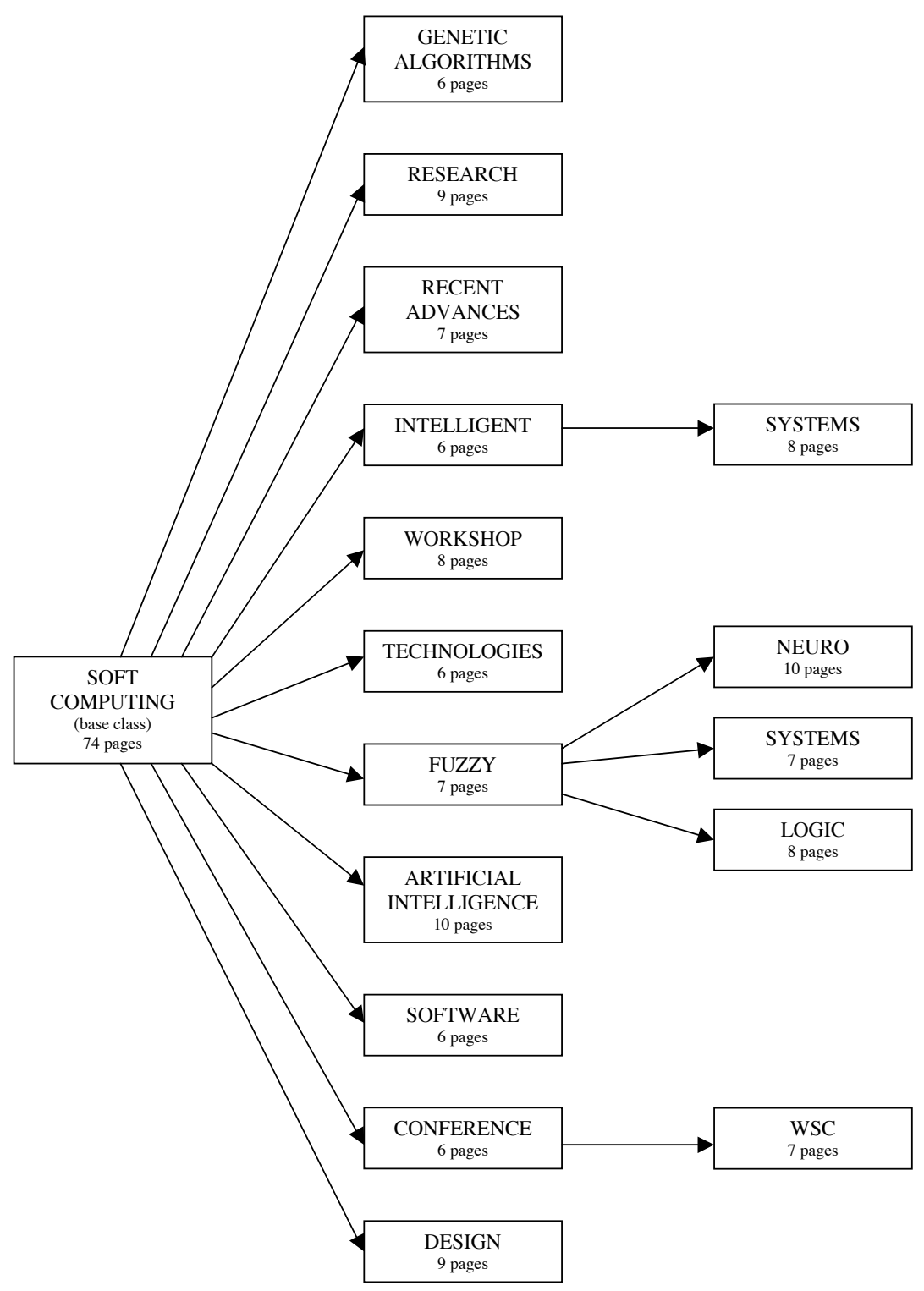

Figure 5.5. Cluster Hierarchy Created by CHCA for the Query "Soft Computing”

This cluster hierarchy is a knowledge representation about a domain (i.e. the topic specified by the query, soft computing) induced from data. So in essence we have performed a web mining task in creating this knowledge representation. But what exactly do we gain from the cluster hierarchy? One thing we discover is, given a topic (query string) as input, what some of the other related topics or sub-topics are. For example, with SOFT COMPUTING we also see such topics as ARTIFICIAL INTELLIGENCE and GENETIC ALGORITHMS. The structure of the hierarchy gives us additional 
knowledge. We see, for example, that the cluster corresponding to FUZZY has children such as LOGIC (fuzzy logic) and NEURO (as in neuro-fuzzy). So these clusters and the pages in them are specializations of the topic FUZZY relating to the sub-topics of fuzzy logic and neuro-fuzzy soft computing, respectively. Conversely, pages of a parent cluster are generalizations of those in the child clusters. Also, sibling clusters (clusters with common parents) are topics related by their parents (e.g. RECENT ADVANCES and RESEARCH).

Table 5.2. Results of the Grouper Custom System for the Query "Soft Computing"

\begin{tabular}{|c|c|}
\hline Cluster Title & Number of Pages in Cluster \\
\hline soft computing strategies in life sciences & 15 \\
\hline fuzzy logic & 40 \\
\hline mammut soft computing ag banking software & 12 \\
\hline genetic algorithm & 19 \\
\hline artificial intelligence & 20 \\
\hline probabilistic reasoning & 14 \\
\hline premier on-line event on soft computing and it & 5 \\
\hline uncertainty and partial truth & 6 \\
\hline partial truth to achieve tractability & 4 \\
\hline fuzzy set & 12 \\
\hline original paper & 9 \\
\hline data mining & 8 \\
\hline takeshi furuhashi & 6 \\
\hline lotfi zadeh & 9 \\
\hline rudolf kruse & 6 \\
\hline neuronale netze & 5 \\
\hline drug discovery & 6 \\
\hline computational intelligence & 8 \\
\hline evolutionary computation & 6 \\
\hline fuzzy control & 10 \\
\hline pattern recognition & 5 \\
\hline current research & 7 \\
\hline rapport with reality & 4 \\
\hline recent advances & 5 \\
\hline technische universit & 4 \\
\hline tolerance for imprecision & 5 \\
\hline nature biotechnology & 4 \\
\hline consortium of methodologies & 4 \\
\hline chaos theory & 5 \\
\hline initiative in soft computing & 8 \\
\hline machine intelligence & 6 \\
\hline on-line tutorial & 4 \\
\hline drug design & 5 \\
\hline machine learning & 5 \\
\hline all others & 83 \\
\hline
\end{tabular}

Like our system, the system of Zamir and Etzioni (called Grouper) also clusters web search results. Grouper uses snippets returned by search engines rather than examining the entire contents of the web documents, so we similarly used snippets with our system for the experiments in this section. One of the main differences with our method is that the clusters created by Grouper's STC algorithm are not arranged in a hierarchy. We tried several searches using the latest publicly available version of the Grouper system (Grouper Custom) [49] and found that our system compared favorably with it. To try and make a fair comparison, we performed a search for the same topic (soft computing) and retrieved a similar number of results (186 with Grouper compared 
to 194 for our system). We also used the best quality search option that is provided by Grouper. The created clusters and the number of pages in each are given in Table 5.2. Note that Grouper allows pages to belong to more than one cluster (non-exclusive clustering), which is why the total is greater than 186 . The results are presented in their original order. Although the sets of pages used by the two systems are not identical, it is not unreasonable to informally compare the clusters created by Grouper (Table 5.2) to the cluster hierarchy created by our system (Fig. 5.5). Both systems produce mostly reasonable clusters, such as GENETIC ALGORITHM(S) and ARTIFICIAL INTELLIGENCE. The major difference is that our system also illustrates the relationships between these clusters. Our system also produces fewer, larger clusters due to the parameters we selected. Grouper seemingly produces too many clusters overall (35 created by Grouper vs. 17 for our system), and some of those clusters appear irrelevant (such as DRUG DESIGN or RAPPORT WITH REALITY). The results of a search for the topic "data compression" are given for our system and Grouper in Fig. 5.6 and Table 5.3, respectively. Like the results for the other topic, our system creates fewer (35 vs. 10 clusters), larger, and generally more relevant clusters than Grouper.

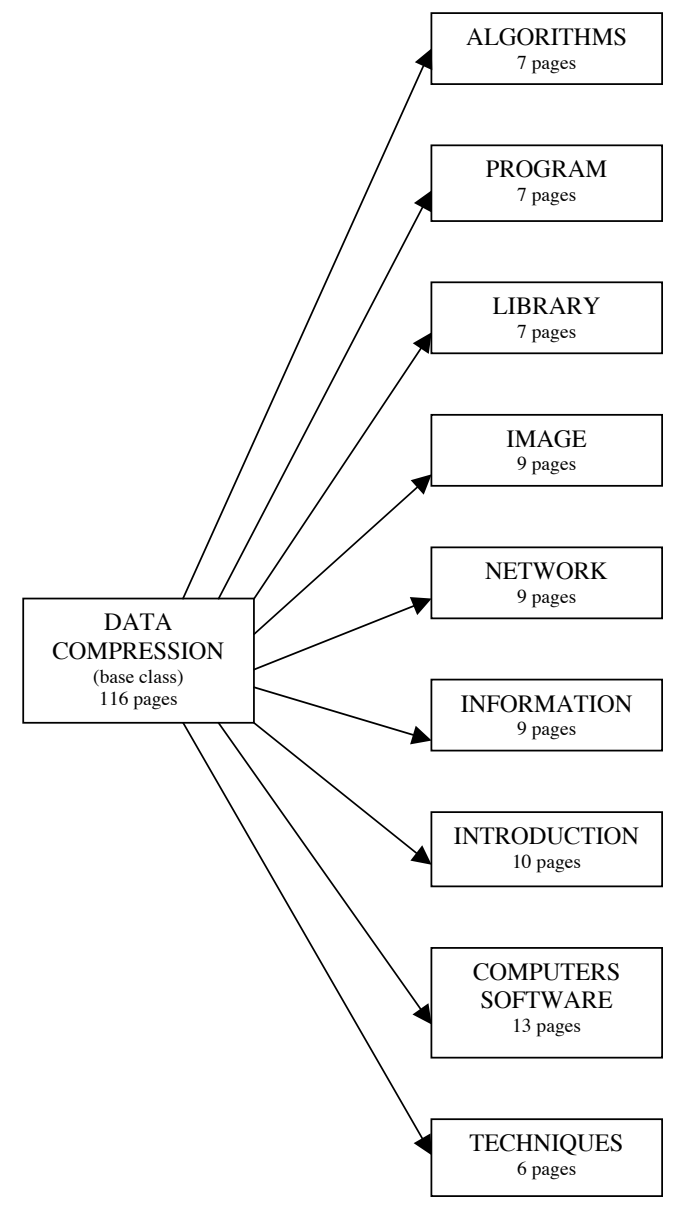

Figure 5.6. Cluster Hierarchy Created by CHCA for the Query "Data Compression" 
Table 5.3. Results of the Grouper Custom System for the Query "Data Compression"

\begin{tabular}{|c|c|}
\hline Cluster Title & Number of Pages in Cluster \\
\hline data compression asp component control library bv & 5 \\
\hline mitsuharu arimura bookmark on source data coding & 4 \\
\hline arithmetic coding & 12 \\
\hline mark nelson & 8 \\
\hline mitsuhara arimura & 7 \\
\hline huffman coding & 10 \\
\hline video teleconference & 8 \\
\hline audio teleconference & 8 \\
\hline compression techniques & 17 \\
\hline submit a url & 5 \\
\hline dobb journal & 5 \\
\hline compression algorithm & 20 \\
\hline source code & 7 \\
\hline calgary corpus & 4 \\
\hline mobile computing & 4 \\
\hline compression ratio & 9 \\
\hline webopedia definition and link & 4 \\
\hline teleconference planner & 4 \\
\hline teleconference phones & 4 \\
\hline signal processing & 4 \\
\hline teleconference calling & 4 \\
\hline lossy compression & 9 \\
\hline teleconference service & 4 \\
\hline fixed length & 4 \\
\hline introduction to data & 11 \\
\hline fewer bit & 4 \\
\hline teleconference equipment & 4 \\
\hline teleconference center & 4 \\
\hline teleconference services & 4 \\
\hline adaptive huffman & 4 \\
\hline image compression & 11 \\
\hline data compression library & 8 \\
\hline original string & 4 \\
\hline lossless data & 9 \\
\hline all others & 103 \\
\hline
\end{tabular}

\subsubsection{Comparison with Vivísimo}

We also compared our system with the commercial Vivísimo system [125], which performs a hierarchical clustering of web search results. We submitted the same 10 query strings to each system (shown in Table 5.4) and compared the resulting cluster hierarchies. The query strings reflect a wide range of topics, from air quality modeling to wedding photography (they were selected from search strings submitted by users of our system). The amount of pages clustered were roughly comparable in each case; for these experiments we used AltaVista with our system, which returned up to 200 pages. Vivísimo also returned a maximum of 200 pages from AltaVista (using the customized search option). Both systems removed duplicate results and worked with snippets (title and description) provided by the search engines in lieu of the full text of the pages. We evaluated the systems by four measurements: 1 . total number of clusters created, 2 . maximum hierarchy depth, 3 . number of base clusters, and 4 . average number of children of base clusters. The results are presented in Table 5.5. In the table, the value in the left column (C) represents our system using CHCA, and the value in the right column (V) 
represents Vivísimo. Vivísimo allows the user to expand the initial hierarchy by clicking on a "More..." link. In our evaluation, we consider only the clusters that are initially displayed by the system. Further, Vivísimo includes unspecified clusters entitled "Other Topics"; these clusters were ignored.

Table 5.4. List of Query Strings Used for Comparison

\begin{tabular}{|c|c|}
\hline Query & String \\
\hline$\# 1$ & nuclear medicine \\
\hline$\# 2$ & linux \\
\hline$\# 3$ & wedding photography \\
\hline$\# 4$ & nursery system \\
\hline$\# 5$ & pentium processor \\
\hline$\# 6$ & human genome project \\
\hline$\# 7$ & scuba diving equipment \\
\hline$\# 8$ & stamp collection \\
\hline$\# 9$ & voice recognition \\
\hline$\# 10$ & air quality modeling \\
\hline
\end{tabular}

Table 5.5. Summary of Comparison for 10 Searches (C: CHCA, V: Vivísimo)

\begin{tabular}{|c|c|c|c|c|c|c|c|c|}
\hline \multirow{2}{*}{ Query } & \multicolumn{2}{|c|}{$\begin{array}{c}\text { Number of } \\
\text { clusters }\end{array}$} & \multicolumn{2}{c|}{$\begin{array}{c}\text { Maximum } \\
\text { depth }\end{array}$} & \multicolumn{2}{c|}{$\begin{array}{c}\text { Number of } \\
\text { base clusters }\end{array}$} & \multicolumn{2}{c|}{$\begin{array}{c}\text { Avg. children/base } \\
\text { cluster }\end{array}$} \\
\cline { 2 - 9 } & $\mathrm{C}$ & $\mathrm{V}$ & $\mathrm{C}$ & $\mathrm{V}$ & $\mathrm{C}$ & $\mathrm{V}$ & $\mathrm{C}$ & $\mathrm{V}$ \\
\hline$\# 1$ & 18 & 30 & 2 & 3 & 1 & 1 & 16 & 10 \\
\hline$\# 2$ & 15 & 18 & 2 & 2 & 1 & 1 & 12 & 10 \\
\hline$\# 3$ & 17 & 35 & 2 & 4 & 1 & 1 & 15 & 10 \\
\hline$\# 4$ & 17 & 35 & 2 & 2 & 10 & 1 & 0.8 & 10 \\
\hline$\# 5$ & 15 & 29 & 2 & 3 & 2 & 1 & 3.5 & 10 \\
\hline$\# 6$ & 14 & 51 & 1 & 3 & 1 & 1 & 13 & 10 \\
\hline$\# 7$ & 15 & 22 & 3 & 2 & 3 & 1 & 0.66 & 10 \\
\hline$\# 8$ & 9 & 25 & 2 & 2 & 1 & 1 & 3 & 10 \\
\hline$\# 9$ & 17 & 28 & 2 & 3 & 1 & 1 & 14 & 10 \\
\hline$\# 10$ & 12 & 48 & 3 & 3 & 5 & 1 & 0.6 & 10 \\
\hline Mean & 14.9 & 32.1 & 2.1 & 2.7 & 2.6 & 1.0 & 7.86 & 10.0 \\
\hline
\end{tabular}

We can make the following observations from the data and our personal experience from using the system:

(1) Vivísimo creates more clusters than our system. This can be observed directly from the total number of clusters for each query, as well as from the mean. The more clusters in the hierarchy, the more difficult it is to browse.

(2) Vivísimo always created a single base cluster, which corresponds to the topic specified by the search string. In contrast, our method creates base clusters as appropriate to each search (i.e. the search topic is not necessarily the root). Consider query \#7, which is "scuba diving equipment." Our system created 3 base clusters for this query: "scuba," "diving," and "equipment" and then created the 
necessary combinations (e.g. "scuba diving") though multiple inheritance. This makes sense since the search returned, for example, pages that contained the term "diving" but not the terms "scuba" and "diving" together. For query \#4, "nursery system," our system created ten base clusters. This seems reasonable given the rather vague nature of the query; search engines returned pages on everything from health care to plants for this query. Thus, our system does not force the search topic to appear at the root (although it can appear at the root when appropriate, e.g. query \#2 "linux"), which allows for the investigation of more general topics as well as those that are more specific.

(3) Vivísimo created a fairly constant number of children for base clusters. Recall that we did not examine all the results for Vivísimo, only the initially displayed default results. This appears to limit the number of second level clusters to a constant number.

(4) Comparing the average maximum depths from each system, we see that our method creates a shallower hierarchy than Vivísimo. Thus less "digging" to reach clusters at lower levels is required for our system.

(5) By subtracting the number of base clusters and number of children of base clusters (computed by multiplying the number of base cluster by the average number of children of base clusters) from the total number of clusters, we can derive the number of clusters residing at the lower hierarchy levels. By comparing this number with the total number of clusters and the hierarchy depth, we see that our system often placed most of the clusters at the top two levels (base clusters and their children). On the other hand, Vivísimo had many clusters residing at the lower levels, leading to hierarchies that often produced many branches and that were very wide at the lower levels. Such hierarchies are difficult to examine since they either require much "digging" and backtracking (in the depth-first sense) or they require displaying increasingly many nodes at each level (in the breadth-first sense).

(6) Vivísimo's hierarchies were much slower to browse than those of our system. This is probably due to the fact that Vivísimo includes the pages themselves in the hierarchy display, leading to longer loading and rendering times. Our system includes only clusters in the hierarchy; pages are displayed separately.

\subsection{Graph Hierarchy Construction Algorithm (GHCA)}

In this section, we describe a graph-based version of CHCA, GHCA. GHCA performs clustering using graph representations of web documents rather than the binary term-vector representations we previously used for CHCA. Both the input (the web documents) and the output (the clusters) are represented by graphs. In sub-section 5.5.1 we give an explanation of the user provided algorithm parameters. In sub-section 5.5.2 we describe the pre-processing used to transform the web documents into their graph 
representations. We present the Graph Hierarchy Construction Algorithm (GHCA) in detail in sub-section 5.5.3.

(PP.1) Given the set of relevant web pages provided by search engines, download the full text of each page.

(PP.2) Parse the text for each web document and create page graphs using the standard representation (see Section 4.2).

(PP.3) Optionally create separate small keyword graphs for each page based only on the title web document section as described in Section 4.2 (this is the default option).

(PP.4) Optionally remove stop words (e.g. "is", "the", etc.) from the page graphs and the keyword graphs by deleting the nodes corresponding to the stop words and their incident edges (default option, see Section 4.1).

(PP.5) Optionally perform stemming by conflating grammatical variants of terms to the most commonly occurring form (default option, see Section 4.1).

(PP.6) Perform dimensionality reduction using one of the following methods:

(a) Reduce the set of possible terms to the MTT terms which appear on the most pages; delete nodes not in this set and their incident edges from all graphs (default method).

(b) Reduce each graph to the $M T T$ most frequently occurring terms on that page; delete the infrequently occurring nodes and their incident edges.

(PP.7) Optionally remove edges from the page graphs which occur infrequently (i.e., less than 3 times); the default is not to remove edges. Note that edges will probably occur infrequently in keyword graphs, so we do not apply this option when using keyword graphs.

(PP.8) Add either the keyword graphs (the default) or the page graphs to the set of candidate graphs.

(PP.9) Optionally generate the maximum common subgraphs of each possible pair of candidate graphs, and add them to the set of candidate graphs (default option).

(PP.10) Optionally, for each candidate graph, if the graph contains at least one edge (meaning the graph contains a phrase), delete nodes with no incident edges (i.e. extraneous isolated nodes) from that graph, since phrases usually convey more useful information than single-word terms. If there are no phrases, the graph is left as-is (default option).

(PP.11) Remove all duplicate graphs from the set of candidate graphs, leaving only a set of unique graphs. This means that two distinct documents represented by identical graphs are assumed to be related to the same topic.

Figure 5.7. Pre-processing Phase of GHCA

\subsubsection{Parameters}

GHCA uses five user-provided parameters to control the properties of the resulting cluster hierarchy. Most of these are identical to the CHCA parameters, however the updated version of our system that utilizes GHCA allows the user many new options 
relating to these parameters. The algorithm parameters are given below, along with their default values (which were determined experimentally across a variety of different searches):

(1) MTT (Maximum Terms Threshold). This parameter restricts the maximum number of vertices in the resulting graph representations of documents. We have two options. We can use the MTT most frequently occurring terms on each page (where frequency means the number of occurrences on a given page). Or we can create a common set of the MTT most frequently occurring terms across all pages (where frequency means the number of pages where a term occurs at least once). The default option is to use the 30 most frequently occurring terms across all pages.

(2) MPT (Minimum Pages Threshold). This parameter is used in the pruning section of GHCA by removing clusters that have fewer than MPT native pages assigned to them. The default value is 3 .

(3) MDT (Maximum Distance Threshold). This parameter is used for restricting the growth of the hierarchy. We do not add clusters to the hierarchy whose difference in size from their parent(s) is greater than $M D T$. As we mentioned in Chapter 4, the size of a graph is defined as the sum of the number of edges and vertices in the graph. The default value of $M D T$ is 2 , which is large enough to allow the addition of one new term to an existing phrase (i.e. one node and one edge).

(4) MCT (Maximum Cluster Threshold). This parameter is used to limit the overall size of the hierarchy. We stop the hierarchy construction phase of the algorithm once it has created $M C T$ clusters or we have no candidate graphs remaining. The default value is 50 .

(5) BCST (Base Cluster Size Threshold). This parameter is used to limit the size of base (top level) clusters. We do not create a new base cluster if its size exceeds $B C S T$. The default value of $B C S T$ is 3 . The default value is large enough to admit a two term phrase (i.e. two nodes connected by an edge) as a base cluster.

\subsubsection{Graph Creation and Pre-processing}

The general procedure for creating graphs from web documents and preprocessing is described in Fig. 5.7. The procedure of creating graphs from web documents is similar to what we described in Chapter 4, however some steps are now optional (such as stemming) and we create optionally a separate set of graphs based only the title section information (see Section 4.2). 
(IHC.1) Find the candidate graph with minimum size, where the size of a graph $G,|G|$, is defined as the sum of the number of edges and vertices in the graph, $|V|+|E|$, and select it to be the cluster candidate. In case of a tie, select one of the graphs at random.

(IHC.2) Determine the possible parents of the cluster candidate in the hierarchy, such that any parents of the cluster candidate are subgraphs of the cluster candidate and the distance, defined as the difference in size between the two graphs, is minimum.

(IHC.3) If the cluster candidate is a base cluster (it has no parents in step (IHC.2)) and the size of the graph is less than or equal to $B C S T$, add the cluster candidate as a base cluster in the hierarchy.

(IHC.4) If the cluster candidate is not a base cluster and the difference in size between the cluster candidate and its parent(s) is less than or equal to $M D T$, add the cluster candidate to the hierarchy.

(IHC.5) Remove the cluster candidate from the set of candidate graphs.

(IHC.6) While the number of clusters in the hierarchy is less than $M C T$ and there are still candidate graphs remaining, go to step (IHC.1); otherwise, proceed to the initial document assignment phase.

\section{Figure 5.8. Initial Hierarchy Construction Phase of GHCA}

\subsubsection{Graph Hierarchy Construction Algorithm (GHCA)}

Given the set of candidate graphs from the previous section, we can perform the Graph Hierarchy Construction Algorithm. The basic steps are initial hierarchy construction (Fig. 5.8), document assignment (Fig. 5.9), and bottom-up cluster pruning (Fig. 5.10).

For every page represented in the set of page graphs, determine what clusters in the hierarchy have the smallest distance according to the MCS distance measure (Eq. 3.3).

(DA.1) If the minimum distance is 1, skip this page (go back to step DA.1).

(DA.2) Assign the page to the cluster(s) which have minimum distance as a native page.

(DA.3) Also assign the page to super-clusters above the clusters selected in step (DA.2) in the hierarchy as an inherited page; continue to propagate the inherited page up the hierarchy from child to parent until a base cluster is reached.

\section{Figure 5.9. Document Assignment Phase of GHCA}

The initial hierarchy construction procedure is given in Fig. 5.8. The purpose of this phase of GHCA is it create the initial cluster hierarchy. As mentioned in Chapter 4, the definition of size given in step IHC. 1 is necessary because it may be the case that we have, for example, a cluster represented by a graph with 2 vertices and no edges (e.g. DATA, MINING) and a cluster represented by a graph with 2 vertices and one edge $(\mathrm{DATA} \rightarrow \mathrm{MINING})$. The first cluster represents pages where the terms "data" and "mining" occur with no particular relationship on the same web document; the second 
represents pages where the phrase "data mining" occurs (i.e., there is a more specific relationship, the terms appear adjacent to each other in the specified order). If we consider only the number of nodes in computing the size of the graph, these clusters are considered to be equally similar when calculating the distance. This is undesirable because the choice of assignment becomes arbitrary when clearly each graph represents a different concept.

After initial hierarchy construction, the document assignment phase of GHCA (Fig. 5.9) is performed. The purpose of this phase is to assign web documents to the clusters created in the initial hierarchy construction phase. The previous version of our system (described above in sub-section 5.2.3) assigned each web page to only one cluster (exclusive assignment), using tie breaking methods when the distance measures for two or more clusters were equal. We felt this was unnecessarily restrictive, so we have allowed non-exclusive assignment of pages. In addition, base clusters in the induced hierarchy of graphs will have more pages (native + inherited) assigned to them than their descendant clusters; the clusters in the bottom level will represent the most specific topics described by the fewest number of pages.

(CP.1) Starting with the lowest level in the hierarchy, delete all clusters at that level from the hierarchy that have less than MPT native pages assigned to them.

(CP.2) Given the new hierarchy, re-assign all the pages from the deleted clusters as described above in steps (DA.1) to (DA.3).

(CP.3) Fix orphaned clusters by updating the parent information as in step (IHC.2) of the initial hierarchy construction.

(CP.4) Repeat steps (CP.1) to (CP.3) going up one level in the hierarchy each iteration until the top level is reached.

Figure 5.10. Cluster Pruning Phase of GHCA

After initial document assignment, the last phase of GHCA is bottom-up cluster pruning (Fig. 5.10). This removes clusters with less than MPT web documents assigned to them, starting with the lowest level clusters.

(R.1) For each cluster, first display the longest simple paths (acyclic paths not contained in any other acyclic paths) in the graph as ordered phrases; next show any isolated nodes as single terms.

(R.2) If the cluster is not a base cluster, show only those phrases or terms which are specific to the graph (i.e., those not displayed for a parent cluster).

\section{Figure 5.11. Results Display Methodology for GHCA}

We use a different method of displaying the terms associated with each cluster than we used for vector-based CHCA. Since the graphs used in GHCA preserve the term ordering information, we can display phrases (groups of related terms) to the user instead 
of an alphabetical list of single terms. In order to display the phrases and terms represented by each cluster graph to the user, we use the approach described in Fig. 5.11. For brevity in the output we omit the edge labeling information about the document sections where the terms or phrases were found.

Note that the first three phases above, initial hierarchy construction, initial document assignment, and bottom-up cluster pruning, are directly analogous to Steps 3 , 4, and 5, respectively, in CHCA. The following straightforward extensions are performed to go from binary vector-based CHCA to graph-based GHCA:

(1) Instead of the size being determined by the Hamming Weight (the number of 1 bits), the size is determined by the number of nodes and edges in a graph.

(2) Instead of using a bitwise AND operation to determine the subset relationship, we determine if one graph is a subgraph of another directly through the subgraph relationship.

(3) The distance measure used to assign pages to clusters is now Eq. 3.3 rather than Eq. 5.1.

Although there are other changes between CHCA and GHCA as presented here, those are new options or refinements to the search system (such as allowing non-exclusive assignments) rather than changes to the fundamental CHCA algorithm itself. With only the three changes listed above we were able extend the algorithm to work with more complex graphs rather than the simpler bit vectors we used previously.

\subsubsection{GHCA Examples}

In Fig. 5.12 we show the results of performing four different searches for our system with GHCA using the default parameter values and options; the searches are for "data mining" (top left), "soft computing" (top right), "graph theory" (bottom left), and "scuba diving equipment" (bottom right). The total number of pages assigned to each cluster is shown in parentheses. Sub-clusters are indicated by indentation. The phrases and terms associated with each cluster are determined using steps (R.1) and (R.2) in Fig. 5.11 above. For example, the first cluster in the "data mining" hierarchy is displayed as the phrase "data management", meaning the graph contained two nodes ("data" and "management") and an edge from "data" to "management." Distinct phrases or terms are separated by commas. Note that this is an improvement over the case of CHCA, which contained no term ordering or phrase information. Under CHCA, which ordered terms for each cluster alphabetically, we would have clusters identified as "computing soft" or "diving equipment scuba." With GHCA the correct order can be preserved: "soft computing" and "scuba diving equipment." GHCA also allows differentiation of clusters based on the phrase information; e.g. "DATA, MINING" is separate cluster from "DATA MINING." 


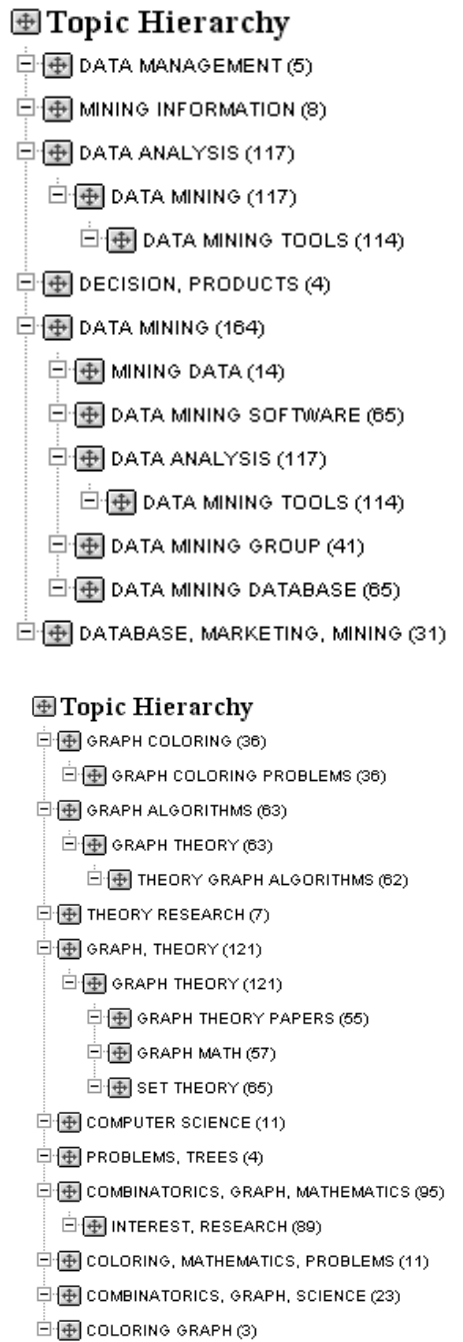

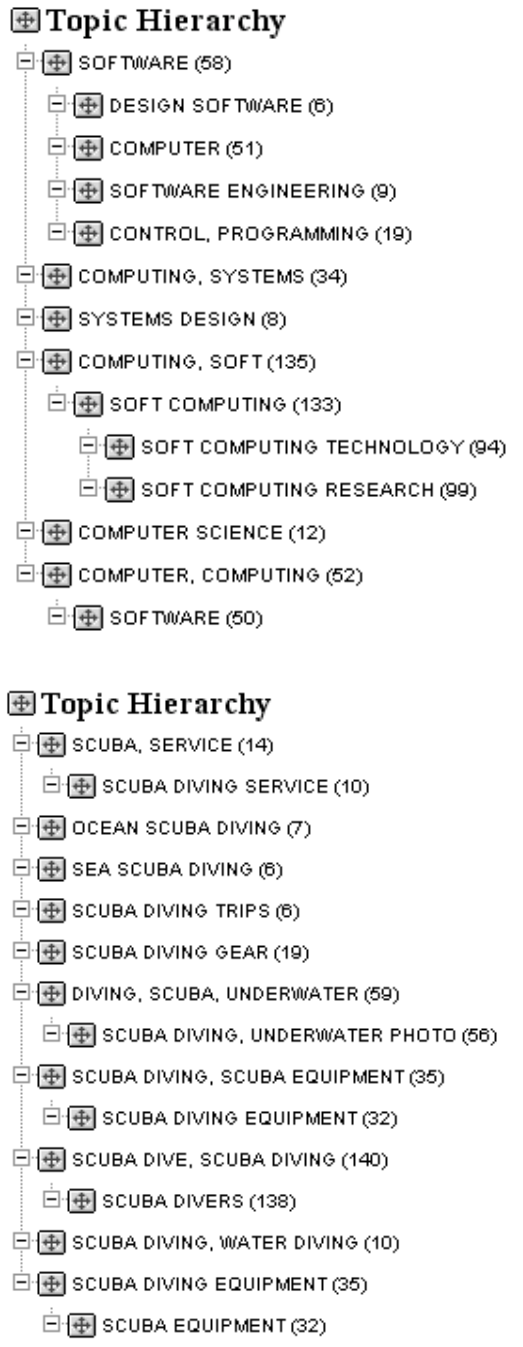

Figure 5.12. Examples of Cluster Hierarchies Generated by GHCA

\subsection{Comments}

In this chapter we presented a system designed to better organize the results returned by conventional web search engines. Novel hierarchical clustering algorithms called CHCA (Cluster Hierarchy Construction Algorithm) and GHCA (Graph Hierarchy Construction Algorithm) were employed to hierarchically cluster web pages by topic using the concept of inheritance. The cluster hierarchy generated by the algorithm can be viewed as a knowledge representation about the domain that has been induced from the web content data. In other words, CHCA and GHCA are web mining algorithms. By creating a cluster hierarchy from the results we are able to cluster web pages as well as determine relationships between the topics related to the search. GHCA modeled web pages as graphs, and worked directly on these representations when clustering. The additional information included ordered phrases; CHCA's vector representation had only alphabetically ordered lists of atomic terms. This allows for better differentiation among 
the clusters (i.e. whether the terms appear together as a phrase, indicating a specific relationship) and for more coherent output of cluster names (the term ordering information is preserved).

The results (the cluster hierarchies) from actual searches show how our system differs from other web search clustering systems. Here is a summary of the main contributions of this chapter:

(1) As shown by the examples of the system output and comparisons with similar systems, our system produces a reasonable and useful clustering of the web pages. Further, the behavior of our system is in-line with clusterings of web pages produced manually by humans when comparing full text processing to snippets.

(2) CHCA and GHCA include several parameters which allow us to direct the characteristics of the cluster hierarchy. This is important since other hierarchical clustering methods, such as the popular agglomerative hierarchical clustering algorithm, can produce very large trees which may be difficult to view and browse. With proper parameters we can achieve a hierarchy of a manageable width and depth. There are versions of the agglomerative hierarchical method that include stopping criteria, but their parameters are topic sensitive [139]. In contrast, our methods' parameters are more sensitive to the search engines used and not to the topics being searched for. We found that when using different search engines we needed to modify the parameters slightly to account for the number of pages returned and the size and content of the snippets returned by the engine. However, once we arrived at a reasonable set of parameter values for a particular search engine, the results tended to be fairly uniform across searches.

(3) We produce not just a series of nested partitionings, as in conventional hierarchical clustering methods, but a clustering with relationships that include generalization (child to parent), specialization (parent to child) and similarity (between siblings). As a result, we can make statements such as when a parent/child relationship exists, the topic of the child cluster is a specialization of the parent cluster and conversely the topic of the parent cluster is a generalization of the child cluster. Sibling clusters that share common parents are related by the topic of their common parent, i.e. they are different specializations of the same cluster. Hierarchies created with conventional hierarchical clustering methods such as the agglomerative and divisive approaches only give us an indication of which clusters include the objects of other clusters (the parent/child relationship) and the similarity between clusters (the sibling relationship). Further, with CHCA and GHCA we are also provided with knowledge of which terms and phrases are used to differentiate and specialize clusters. This gives us an indication of which terms or phrases are most general and how they are used to cause specialization. Such a cluster hierarchy is related to knowledge representation models like frames and semantic networks and by inducing the knowledge from the web content data in effect a web content mining task has been performed. 
(4) We do not use any kind of term frequency per document measure except for pre-processing and dimensionality reduction. Thus those pages with intentionally skewed term frequencies intended to improve their ranking are treated the same as well behaved documents.

(5) CHCA and GHCA are applicable to regular text documents in addition to hypertext (linked) documents. Some web mining approaches only consider links (web structure) and thus are not applicable to text documents.

(6) Our system allows asynchronous search. This permits the user to perform other tasks while his/her request is processed and makes possible the examination of the original full content of web pages rather than the snippets stored by search engines, which may be "stale" (out of date).

After observing the straightforward and simple extensions that allowed the use of web content data represented by graphs instead of vectors in our clustering algorithm, we turned our attention to performing similar extensions with other, well-known machine learning methods for the purpose of web content mining. The results of these investigations begin with the next chapter. 


\section{Chapter Six}

\section{A Graph-Theoretical Extension of the $k$-Means Clustering Algorithm}

\subsection{Introduction}

In this chapter we will introduce a new method of clustering where the data to be clustered will be represented by graphs instead of vectors or other models. Specifically, we will extend the classical $k$-means clustering algorithm to work with graphs that represent web documents. We wish to use graphs because they can allow us to retain information which is often discarded in simpler models. For example, when representing web documents by graphs instead of vectors we can keep information such as the term appearance order or where in the document the terms appear. This in turn can possibly lead to an improvement in clustering quality, and we will investigate this experimentally. In this chapter we will describe the three data sets we will use in our experiments and the measures we will use for comparing clustering performance.

Clustering with graphs is well established in the literature. However, the traditional paradigm in those methods has been to treat the entire clustering problem as a graph: nodes represent the items to be clustered and weights on edges connecting two nodes indicate the distance (dissimilarity) between the objects the nodes represent. The usual procedure is to create a minimum spanning tree of the graph and then remove the remaining edges with the largest weight in the minimum spanning tree until the number of desired clustered (connected components) is achieved [59][123][138]. After applying the algorithm the connected components indicate which objects belong to which clusters: objects whose nodes are connected by edges are in the same cluster. Recently there has been some progress with performing clustering directly on graph-based data. For example, an extension of self-organizing maps (SOMs) which allows the procedure to work with graphs has been proposed [50]; graph edit distance and weighted mean of a pair of graphs were introduced to deal with graph-based data under the SOM algorithm. Clustering of shock trees using tree edit distance has also been considered [75]. Both of these methods have in common that they use graph (or tree) edit distance for their graph distance measures. One drawback of this approach is that the edit cost functions must be specified for each application. Sanfeliu et al. have investigated clustering of attributed graphs using their own function-described graphs as cluster representatives [109]. However, their method is rather complicated and much more involved than our straightforward extension of a classical, simple clustering algorithm.

The chapter is organized as follows. In Section 6.2, we extend the classical $k$ means algorithm to use graphs instead of vectors. In Section 6.3 we will describe the web data sets that we use for our experiments. The three performance measures we use to evaluate performance are given in Section 6.4. In Section 6.5 we present experimental 
results and a comparison with previously published results from clustering the same web document collection when using a vector model and the classical $k$-means algorithm. Concluding remarks are given in Section 6.6.

\subsection{The Extended $k$-Means Clustering Algorithm}

With our formal notation for graphs (Chapter 3), we are ready to describe our framework for extending the classical $k$-means clustering method. The extension is surprisingly simple. First, any distance calculations between data items is accomplished with a graph-theoretical distance measure, such as those of Eqs. 3.3-3.7. Second, since it is necessary to compute the distance between data items and cluster centers, it follows that the cluster centers (centroids) must also be graphs. Therefore, we compute the representative "centroid" of a cluster as the median graph of the set of graphs in that cluster (Eq. 3.27).

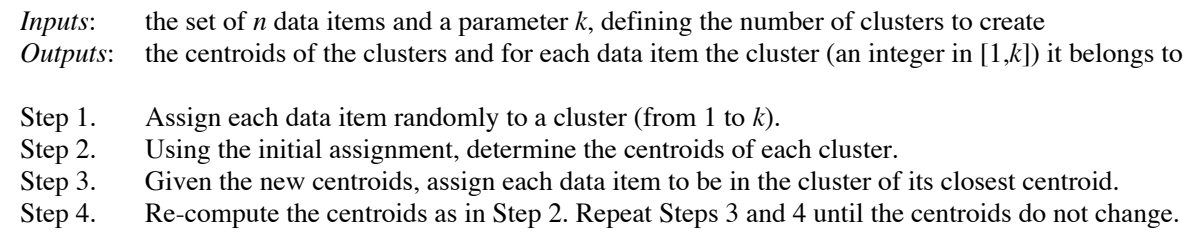

\section{Figure 6.1. The Traditional $k$-Means Clustering Algorithm}

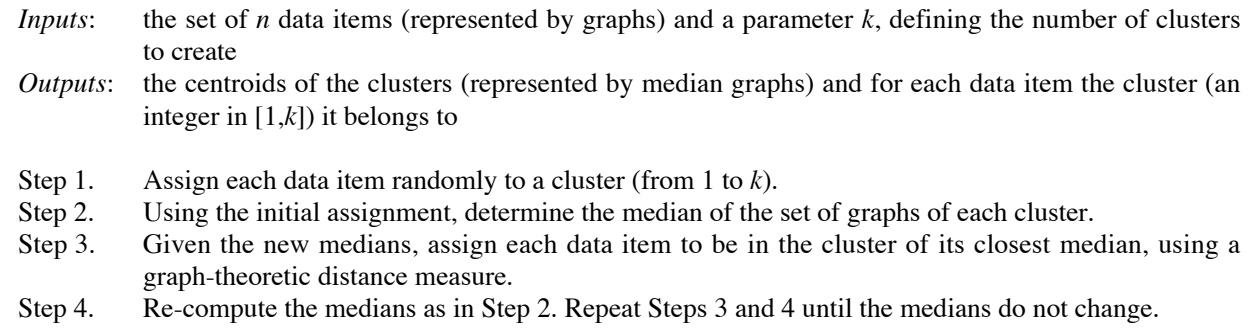

\section{Figure 6.2. The Graph-Theoretic $k$-Means Clustering Algorithm}

The $k$-means clustering algorithm is a simple and straightforward method for clustering data [91]. The basic algorithm is given in Fig. 6.1. This method is applicable to purely numerical data when using Euclidean distance and centroid calculations. The usual paradigm is to represent each data item, which consists of $m$ numeric values, as a vector in the space $\mathfrak{R}^{m}$. In this case the distances between two data items are computed using the Euclidean distance in $m$ dimensions and the centroids are computed to be the mean or weighted mean of the data in the cluster. However, now that we have a distance measure for graphs (Eqs. 3.3-3.7) and a method of determining a representative of a set of graphs (the median, Eq. 3.27) we can apply the same method to data sets whose elements are 
graphs rather than vectors by: 1. replacing the distance measure used in Step 3 with a graph-theoretical distance measure and 2. replacing the centroid computed in Step 2 with the median of a set of graphs. The graph-theoretical version of the $k$-means algorithm is given in Fig. 6.2.

\subsection{Web Document Data Sets}

In order to evaluate the performance of our methods we will perform several experiments using three different collections of web documents, called the $F$-series, the $J$-series, and the $K$-series. ${ }^{*}$ These three data sets were selected because of two major reasons. First, all of the original HTML documents are available for these data sets, which is necessary if we are to represent the web documents using graphs; many other document collections only provide a pre-processed vector representation, which is unsuitable for use with our method. Second, ground truth assignments are provided for each data set, and there are multiple classes representing easily understandable groupings that relate to the content of the documents. Some web document collections are not labeled or are presented with some other task in mind than content-related classification (e.g. building a predictive model based on user preferences).

The F-series originally contained 98 documents belonging to one or more of 17 sub-categories of four major category areas: manufacturing, labor, business \& finance, and electronic communication \& networking. Because there are multiple sub-category classifications from the same category area for many of these documents, we have reduced the categories to just the four major categories mentioned above in order to simplify the problem. There were five documents that had conflicting classifications (i.e., they were classified to belong to two or more of the four major categories) which we removed, leaving 93 total documents. The J-series contains 185 documents and ten classes: affirmative action, business capital, information systems, electronic commerce, intellectual property, employee rights, materials processing, personnel management, manufacturing systems, and industrial partnership. We have not modified this data set. The K-series consists of 2,340 documents and 20 categories: business, health, politics, sports, technology, entertainment, art, cable, culture, film, industry, media, multimedia, music, online, people, review, stage, television, and variety. The last 14 categories are sub-categories related to entertainment, while the entertainment category refers to entertainment in general. These were originally news pages hosted at Yahoo (www.yahoo.com). Experiments on this data set are presented in [120], and we will compare our method to the previously reported results in Section 6.5.

Data for the vector-based experiments we will perform in Chapters 7-10 comes from pre-processed term-document matrices obtained from the same web site that hosts these document collections. The number of dimensions (terms) used for the vector representation of each data set is $332(\mathrm{~F}), 474(\mathrm{~J})$ and 1,458 $(\mathrm{K})$.

* The data sets are available under these names at: ftp://ftp.cs.umn.edu/dept/users/boley/PDDPdata/ 


\subsection{Clustering Performance Measures}

We will evaluate clustering performance in our experiments using the following three clustering performance measures. The first two indices measure the matching of obtained clusters to the "ground truth" clusters (i.e. accuracy), while the third index measures the compactness and separation of the clusters. The first index is the Rand index [65][103], which is defined as:

$$
R_{I}=\frac{A}{A+D}
$$

where $A$ is the number of "agreements" and $D$ is the number of "disagreements" (described below). We compute the Rand index by performing a pair-wise comparison of all pairs of objects in the data set after clustering. If two objects are in the same cluster in both the "ground truth" clustering and the clustering we wish to measure, this counts as an agreement. If two objects are in different clusters in both the ground truth clustering and the clustering we wish to investigate, this is also an agreement. Otherwise, there is a disagreement. Thus the Rand index is a measure of how closely the clustering created by some procedure matches ground truth (i.e. it is a measure of clustering accuracy). It produces a value in the interval $[0,1]$, with 1 representing a clustering that perfectly matches ground truth.

The second performance measure we use is mutual information [23][120], which is defined as:

$$
\Lambda^{M}=\frac{1}{n} \sum_{l=1}^{k} \sum_{h=1}^{g} n_{l}^{(h)} \log _{k \cdot g}\left(\frac{n_{l}^{(h)} \cdot n}{\sum_{i=1}^{k} n_{i}^{(h)} \sum_{i=1}^{g} n_{l}^{(i)}}\right)
$$

where $n$ is the number of data items, $k$ is the desired number of clusters, $g$ is the actual number of "ground truth" categories, and $n_{i}^{(j)}$ is the number of items in cluster $i$ classified to be category $j$. Note that $k$ and $g$ may not necessarily be equal, which would indicate we are attempting to create more (or fewer) clusters than those that exist in the ground truth clustering. Mutual information represents the overall degree of agreement between the clustering and the categorization provided by the ground truth with a preference for clusters that have high purity (i.e. are homogeneous with respect to the classes of objects clustered). Higher values mean better performance.

The third performance measure we use is the Dunn index [35], which is defined as:

$$
D_{I}=\frac{d_{\min }}{d_{\max }}
$$

where $d_{\min }$ is the minimum distance between any two objects in different clusters and $d_{\max }$ is the maximum distance between any two items in the same cluster. The numerator captures the worst-case amount of separation between clusters, while the denominator 
captures the worst-case compactness of the clusters. Thus the Dunn index is an amalgam of the overall worst-case compactness and separation of a clustering, with higher values being better. It does not, however, measure clustering accuracy compared to ground truth as the other two methods do. Rather it is based on the basic underlying assumption of any clustering technique: items in the same cluster should be similar (i.e. have small distance, thus creating compact clusters) and items in separate clusters should be dissimilar (i.e. have large distance, thus creating clusters that are well separated from each other).

\subsection{Comparison with Published Results}

In order to perform an investigation into the performance and possible benefits of our graph-based approach, we performed experiments that apply the extended $k$-means clustering algorithm using graphs to the K-series document collection described above in Section 6.3. We selected this data set primarily because we wished to compare the performance of our method to previously reported results. In [120] Strehl et al. compared the performance of different vector-based clustering methods for the K-series data set, presenting results for a variety of standard clustering methods, including classical $k$ means. The authors used various similarity measures and mutual information (Eq. 6.2) as a performance metric.

In an attempt to adhere to the methodology of the experiments of Strehl et al., which used the vector model approach, we have selected a sample of 800 documents from the total collection of 2,340 and have fixed the desired number of clusters to be $k=$ 40 (two times the number of categories), which is the same number of clusters used in the original experiment. Strehl et al. used this number of clusters "since this seemed to be the more natural number of clusters as indicated by preliminary runs and visualisation" [120]. The results for our method using different numbers of maximum nodes per graph and the original results from Strehl et al. for vector-based $k$-means and a random baseline assignment are given in Table 6.1 (higher mutual information is better). Each row gives the average of 10 experiments using the same 800 item data sample. The variation in results between runs comes from the random initialization in the first step of the $k$-means algorithm. For these experiments we used the MCS distance measure (Eq. 3.3) and the standard representation described in Chapter 4.

The same performance data is plotted graphically in Fig. 6.3. In Fig. 6.4 we show the execution times for performing a single clustering of the document collection when using 5, 50, 100, and 150 nodes per graph. These results were obtained on a $733 \mathrm{MHz}$ single processor Power Macintosh G4 with 384 megabytes of physical memory running Mac OS X. The clustering took 7.13 minutes at 5 nodes per graph and 288.18 minutes for 150 nodes per graph. Unfortunately, no execution time data is available for comparison from the original experiments in Strehl et al.

Briefly reviewing some of these vector-related distance metrics [107][120] (which will also be used in future experiments), we have the Euclidean distance:

$$
\operatorname{dist}_{E U C L}(x, y)=\sqrt{\sum_{r=1}^{n}\left(x_{i}-y_{i}\right)^{2}}
$$


where $x_{i}$ and $y_{i}$ are the $i$ th components of vectors $x=\left[x_{1}, x_{2}, \ldots, x_{\mathrm{n}}\right]$ and $y=\left[y_{1}, y_{2}, \ldots, y_{\mathrm{n}}\right]$, respectively. However, for applications in text and document clustering and classification, the cosine similarity measure [107] is often used due to its length invariance property. We can convert this to a distance measure by the following:

$$
\operatorname{dist}_{\operatorname{COS}}(x, y)=1-\frac{x \cdot y}{\|x\| \cdot\|y\|}
$$

Here $\bullet$ indicates the dot product operation and II...I indicates the magnitude (length) of a vector. Another popular distance measure for determining document similarity is the extended Jaccard similarity [107], which is converted to a distance measure as follows:

$$
\operatorname{dist}_{J A C}(x, y)=1-\frac{\sum_{i=1}^{n} x_{i} y_{i}}{\sum_{i=1}^{n} x_{i}+\sum_{i=1}^{n} y_{i}-\sum_{i=1}^{n} x_{i} y_{i}}
$$

Table 6.1. Results of Our Experiments Compared with Results from Strehl et al.

\begin{tabular}{|c|c|c|}
\hline Method & Max. Nodes/Graph & $\Lambda^{M}$ (average) \\
\hline Graphs & 150 & 0.2218 \\
\hline Graphs & 120 & 0.2142 \\
\hline Graphs & 90 & 0.2074 \\
\hline Graphs & 75 & 0.2045 \\
\hline Graphs & 60 & 0.1865 \\
\hline Extended Jaccard Similarity & - & 0.184 \\
\hline Pearson Correlation & - & 0.178 \\
\hline Cosine Measure & - & 0.178 \\
\hline Graphs & 45 & 0.1758 \\
\hline Graphs & 30 & 0.1617 \\
\hline Graphs & 15 & 0.1540 \\
\hline Graphs & 5 & 0.1326 \\
\hline Random (baseline) & - & 0.066 \\
\hline Euclidean & - & 0.046 \\
\hline
\end{tabular}

From Fig. 6.3 we see that the mutual information generally tends to increase as we allow larger and larger graphs. This makes sense since the larger graphs incorporate more information. On the figure we indicated the values of mutual information from the original experiments for three out of the five methods from Table 6.1. Euclidean is the classical $k$-means with a Euclidean distance measure (Eq. 6.4). Random baseline is simply a random assignment of data to clusters; it is used to provide a baseline for comparison. We would expect any algorithm to perform better than Random, but we see the Euclidean $k$-means did not. Finally, Jaccard is $k$-means using the extended Jaccard similarity (Eq. 6.6). It was the best performing of all the $k$-means methods reported in the original experiment so we have omitted cosine similarity and Pearson correlation on the chart for clarity. 


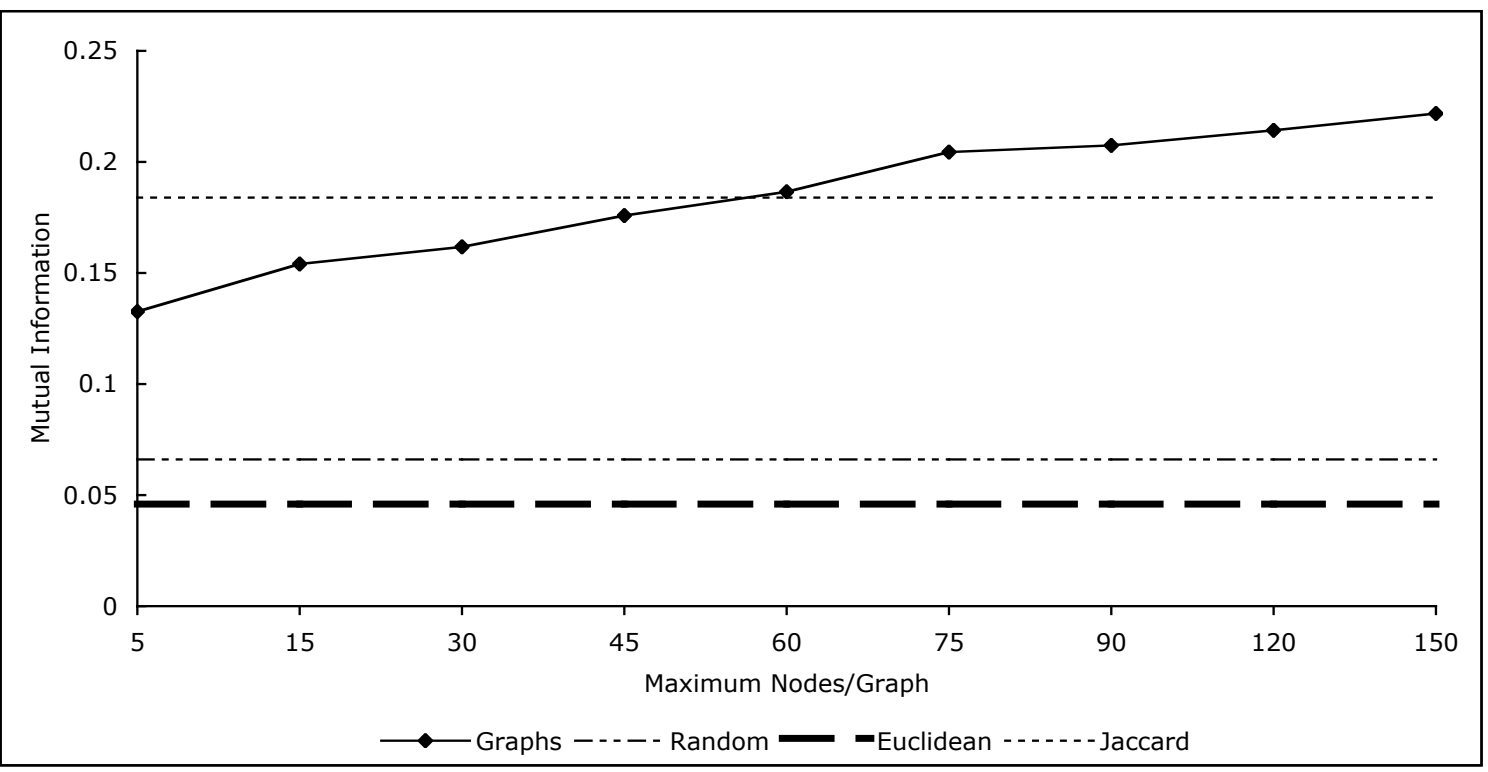

Figure 6.3. Mutual Information as a Function of the Maximum Number of Vertices per Graph

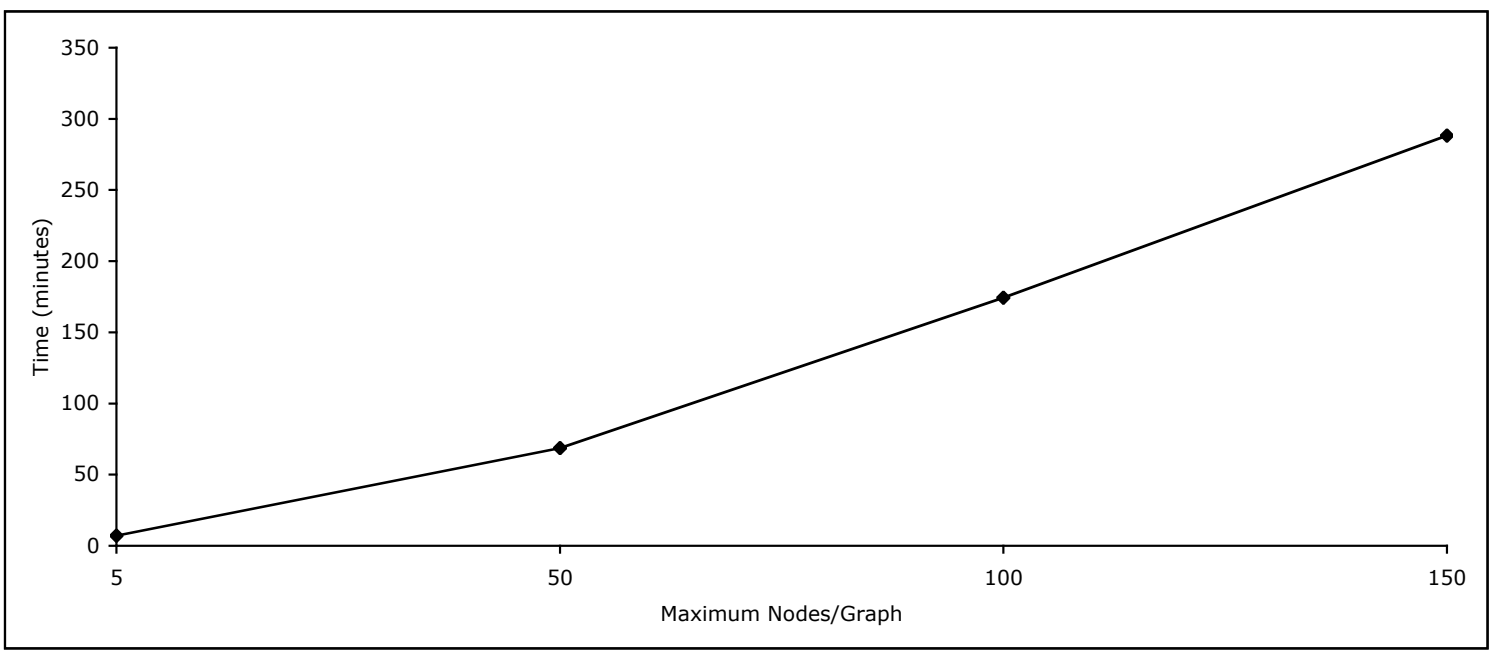

Figure 6.4. Clustering Time as a Function of the Maximum Number of Vertices per Graph

It is not a surprising result to see Euclidean distance perform poorly when using the vector model for representing documents, as it does not have the property of vector length invariance. Because of this, documents with similar term frequency proportions but differences in overall total frequency have large distances between them even though they are supposed to be considered similar. For example, if we were interested in the topic "data mining", a document where the terms "data" and "mining" each appeared 10 times and a document where both terms each appeared 1,000 times are considered to be identical when we have the length invariance property (i.e. their distance is 0 ). It is only 
the relative proportion between the terms that is of interest when determining the document's content, since there are often large variances in total term frequency even for documents related to the same topic. Here both documents contain an equal proportion of the terms "data" and "mining". If the term "mining" occurred much more frequently than "data", we would expect the document to be related to a different topic (e.g., "gold mining"). Under Euclidean distance these two documents would have a large distance (i.e. be considered dissimilar) due to the fact that the difference in total frequency (10 vs. 1,000 ) is large. This is why distance measures with the length invariance property (such as the cosine measure, which measures the cosine of the angle between two feature vectors) are often used in these types of applications in lieu of standard Euclidean distance.

We see that even with only 5 nodes per graph our method outperforms both Euclidean $k$-means and the random baseline; as we increased the number of nodes per graph the performance approaches that of the other $k$-means methods until it exceeded even the best $k$-means method reported at 75 nodes per graph or more. For comparison, the original experiment used a term-document matrix where each vector had 2,903 dimensions. We note a general increasing trend in performance as we allow for larger graphs, which would be consistent with the increase in information that occurs as we introduce new terms (nodes) and phrases (edges) in the graphs. However, the performance improvement is not always strictly proportional with the increase in graph size. For example, the improvement from 60 to 75 is greater than the improvement from 75 to 90 even though we are adding 15 new nodes in each case. This may be due to the fact that the extra nodes added when we increase the graph size, while they are frequently occurring terms, may not always provide information that is useful for discriminating between the documents and in actuality may hinder performance by introducing extraneous data. A future improvement may be to find better methods of selecting the nodes to be used in each graph rather than relying strictly on term frequency.

\subsection{Remarks}

A graph representation allows us to retain structural information such as where terms are located in a document and the order in which terms appear - information which is usually discarded when using the typical vector model approach. Given a graph model of web documents, we can apply classical clustering techniques such as $k$-means by performing a novel extension from vector-based distance and centroid calculations to graph-theoretic distance and median graphs, respectively. To investigate the performance of the extended $k$-means method with our graph representation of web documents, we performed experiments on a web document collection and compared clustering performance with previously reported results of clustering using $k$-means when utilizing a vector model for the same documents. We have discovered the following from our experiments:

(1) Our method outperformed the baseline random assignment method and the vector-based $k$-means method using Euclidean distance, even in the case of maximum dimensionality reduction using only 5 nodes per graph. 
(2) As the maximum number of nodes allowed per graph became larger, the performance of our method generally increased. This reflects an increase in the amount of information in the graphs as we add nodes and edges.

(3) Our method outperformed all the $k$-means clustering methods (Euclidean distance, cosine measure, Pearson correlation, and Jaccard similarity) described in Strehl et al. [120] when we allowed 75 nodes per graph or more. We believe this reflects the information retained by the graph representation which is not present when using the vector model approach.

In the next chapter we will continue to investigate the performance of the graph-theoretic $k$-means algorithm we introduced by performing experiments on other web document collections, varying the graph-theoretical distance measure used in the algorithm, using more clustering performance measures, and using the various methods previously described for representing web documents (Chapter 4). 


\section{Chapter Seven}

\section{Comparison of Different Graph-Theoretical Distance Measures and Graph Representations for Graph-Theoretic Clustering}

\subsection{Introduction}

The experimental results of the previous chapter are encouraging. However, the results reported were based on only a single data set using one clustering performance index. Further, we only implemented one type of graph-theoretic distance measure (the MCS distance) and one graph representation methodology (the "standard"). In this chapter we will further investigate the performance of our graph-based clustering method based on $k$-means by looking at other distance measures and graph representations. We will also apply the method to two other data sets and compare performance with three different performance measures. The typical vector model performance is also reported for a comparison baseline. In Section 7.2 we will give the experimental results when clustering the data sets using five graph-theoretical distance measures. Similarly in Section 7.3 we will look at the effect of graph representations on clustering performance.

We will use the following methodology for the experiments presented in this chapter. For each experiment, whether based on our graph method or a traditional vector method, we will perform ten separate trials each using a random initialization. We will report the average of the ten trials as the performance for that experiment in order to account for the variance between runs due to the random initialization of the clustering algorithm. The vector representation experiments will use the vector-based distance measures (Eq. 6.4-6.6) we described previously. The experiments related to our graphbased methods will be run for a range of maximum graph sizes (the parameter $m$ described in Section 4.2). We will apply the clustering algorithms to our three data sets (Section 6.3) and we will measure clustering performance using the Rand index (Eq. 6.1), mutual information (Eq. 6.2), and the Dunn index (Eq. 6.3). For the experiments in Section 7.2 we will use the MCS, WGU, UGU, MMCS, and MMCSN distance measures (Eqs. 3.3-3.7) with the standard graph representation. Concerning the experiments in Section 7.3, we will use the graph representations (standard, simple, $n$-distance, $n$-simple distance, absolute frequency and relative frequency) defined in Chapter 4 with the MCS distance measure. For the distance related graph representations, namely $n$-distance and $n$-simple distance, we will use $n=5$ (i.e. 5-distance and 5-simple distance). Note that for the graph representations and graph sizes we have defined, $\left|M C S\left(G_{1}, G_{2}\right)\right|=\left|G_{1}\right|+\left|G_{2}\right|-\left|m c s\left(G_{1}, G_{2}\right)\right|$ and thus WGU and MMCSN are identical, as are UGU and MMCS (see Chapter 3). Any differences between the identical distance measures reflected in the results below come from the random initialization of the $k$ means algorithm. 
Table 7.1. Clustering Performance Comparison for K-Series

\begin{tabular}{|c|c|c|c|}
\hline Method & Rand Index & Mutual Information & Dunn Index \\
\hline vector (cosine) & 0.8537 & 0.2266 & 0.0348 \\
\hline vector (Jaccard) & 0.8998 & 0.2441 & 0.0730 \\
\hline graph (40 nodes/graph) & 0.8563 & 0.0752 & 0.0240 \\
\hline graph (70 nodes/graph) & 0.8957 & 0.1174 & 0.0284 \\
\hline graph (100 nodes/graph) & 0.8888 & 0.1310 & 0.0298 \\
\hline graph (200 nodes/graph) & 0.9053 & 0.1618 & 0.0307 \\
\hline
\end{tabular}

In Table 7.1 we give the results of performing $k$-means on the entire K-series data set of 2,340 documents for a variety of graph sizes. We see that while Rand index for the graph methods was similar to the performance of the vector approach, mutual information and Dunn index were not as good. We observe that the performance of all three measures continued to increase with larger graph sizes, so it is possible we created graphs which did not include enough terms for this data set. Recall that in the previous chapter we used a subset of 800 documents from this data set, and performance exceeded that of the best vector method for 60 nodes per graph or more. Another possible explanation for the difference in performance is that there is a variation in the number of clusters created. The previous experiments used $k=40$ for comparison with previously published results. The experiments in this chapter used $k=20$, which matches ground truth.

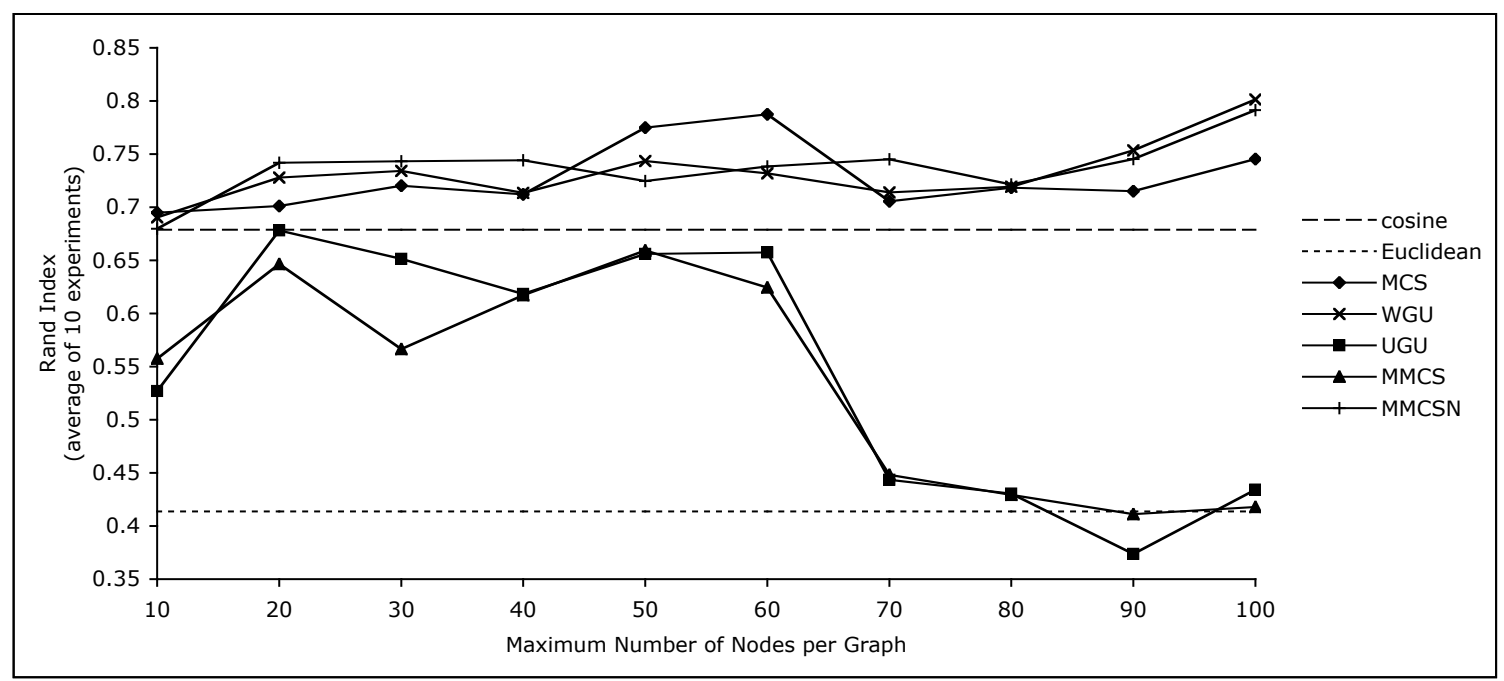

Figure 7.1. Distance Measure Comparison for the F-Series Data Set (Rand Index) 


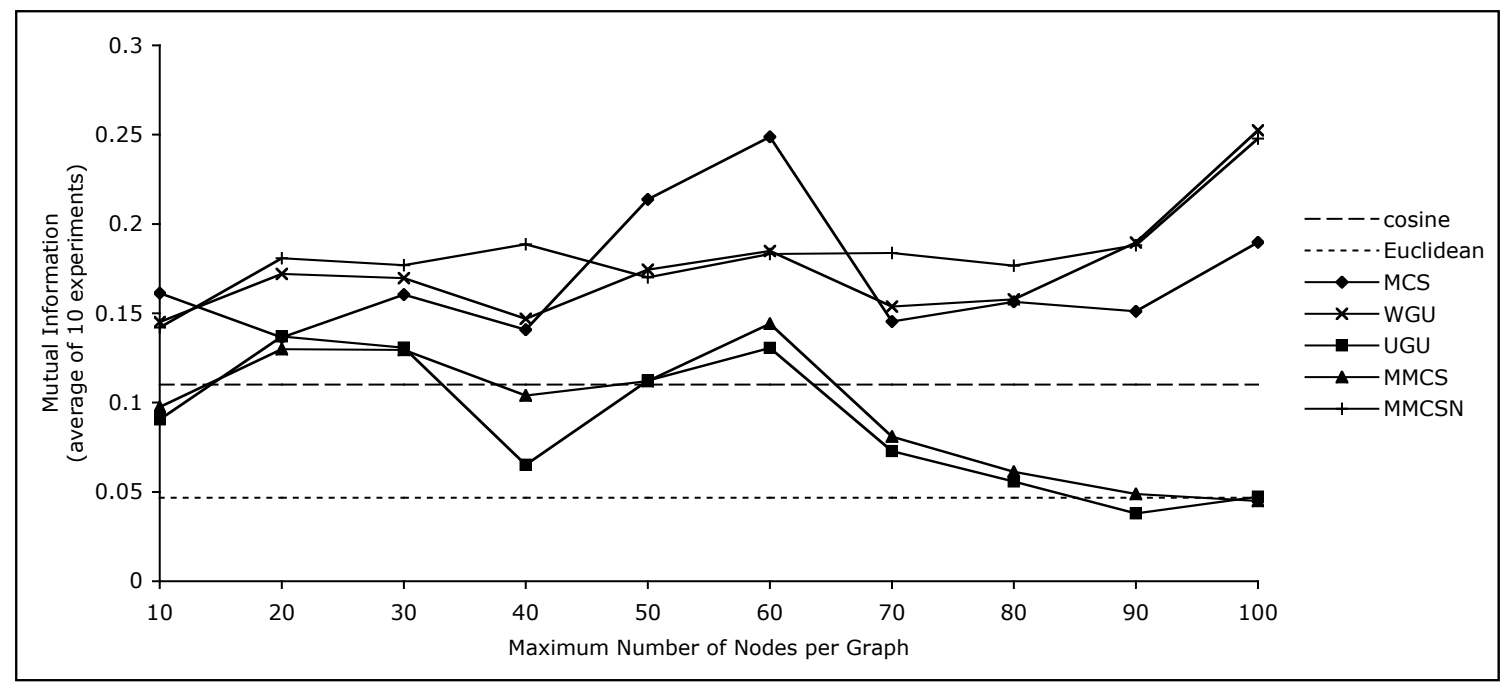

Figure 7.2. Distance Measure Comparison for the F-Series Data Set (Mutual Information)

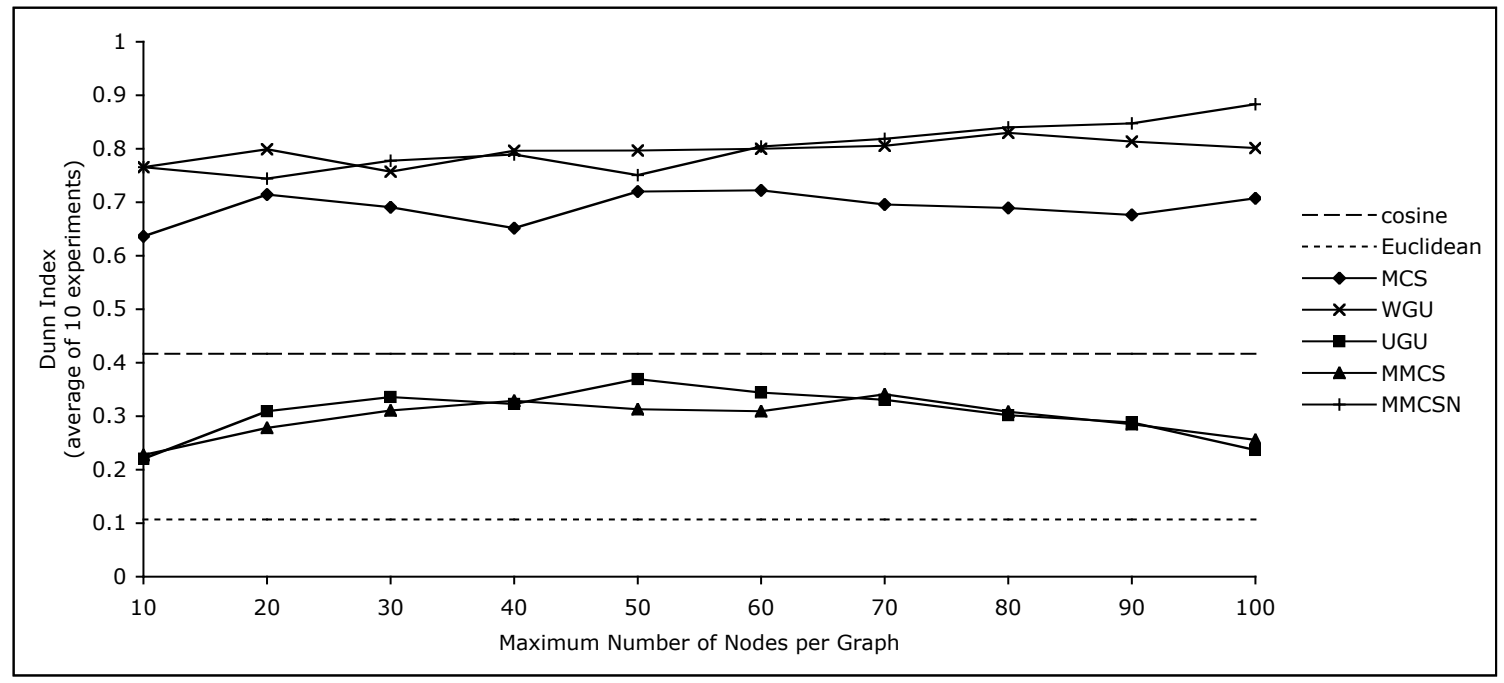

Figure 7.3. Distance Measure Comparison for the F-Series Data Set (Dunn Index)

Table 7.2. Distance Measure Comparison for K-Series

\begin{tabular}{|c|c|c|c|}
\hline Distance Measure & Rand Index & Mutual Information & Dunn Index \\
\hline MCS & 0.8957 & 0.1174 & 0.0284 \\
\hline WGU/MMCSN & 0.8377 & 0.1019 & 0.0385 \\
\hline UGU/MMCS & 0.1692 & 0.0127 & 0.0649 \\
\hline
\end{tabular}




\subsection{Comparison of Distance Measures}

The results of our experiments for the F-series documents are given in Fig. 7.1 for Rand index, Fig. 7.2 for mutual information, and Fig. 7.3 for Dunn index. The results of the vector-model clusterings are shown by horizontal lines. The results for our graphbased method are given for graph sizes ranging from 10 to 100 nodes per graph. Similarly, the results of our experiments for the J-series documents are given in Figs. 7.4-7.6 for graph sizes of 10 to 60 nodes per graph. Results for the K-series, utilizing 70 nodes per graph for each graph-theoretic distance measure, are presented in Table 7.2.

We see that the graph-based methods that use normalized distance measures performed as well or better than vector-based methods using cosine similarity or Euclidean distance. Distance measures that were not normalized to the interval $[0,1]$ performed poorly, particularly when the maximum allowed graph size became large. To see why this occurs, we have provided the following example. Let $\left|G_{1}\right|=10,\left|G_{2}\right|=10$, $\left|m c s\left(G_{1}, G_{2}\right)\right|=0,\left|G_{3}\right|=20,\left|G_{4}\right|=20$, and $\operatorname{lmcs}\left(G_{3}, G_{4}\right) \mid=5$. Clearly graphs $G_{3}$ and $G_{4}$ are more similar to each other than graphs $G_{1}$ and $G_{2}$ since $G_{1}$ and $G_{2}$ have no common subgraph whereas $G_{3}$ and $G_{4}$ do. However, the distances computed for these graphs are $d_{\mathrm{MCS}}\left(G_{1}, G_{2}\right)$ $=1.0, d_{\mathrm{MCS}}\left(G_{3}, G_{4}\right)=0.75, d_{\mathrm{UGU}}\left(G_{1}, G_{2}\right)=20$ and $d_{\mathrm{UGU}}\left(G_{3}, G_{4}\right)=30$. So we have the case that the distance for un-normalized graph union (UGU) is actually greater for the pair of graphs that are more similar. This is both counter-intuitive and the opposite of what happens in the cases of the normalized distance measures. Thus this phenomenon leads to the poor clustering performance. Since the size of the graph includes the number of edges, which can grow at a rate of $O\left(|V|^{2}\right)$, we can see that the potential size variance which causes this phenomenon becomes more pronounced as we increase the number of nodes per graph (i.e. as $|V|$ increases).

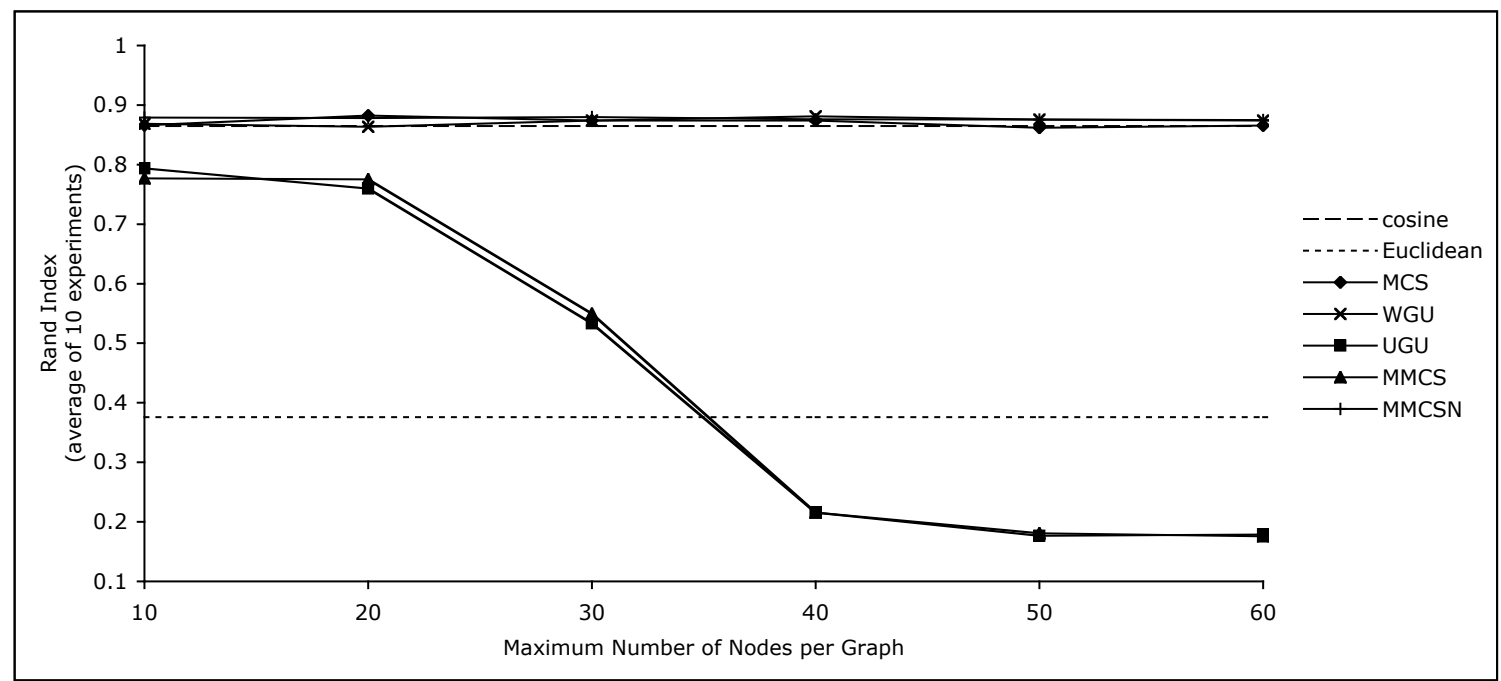

Figure 7.4. Distance Measure Comparison for the J-Series Data Set (Rand Index) 


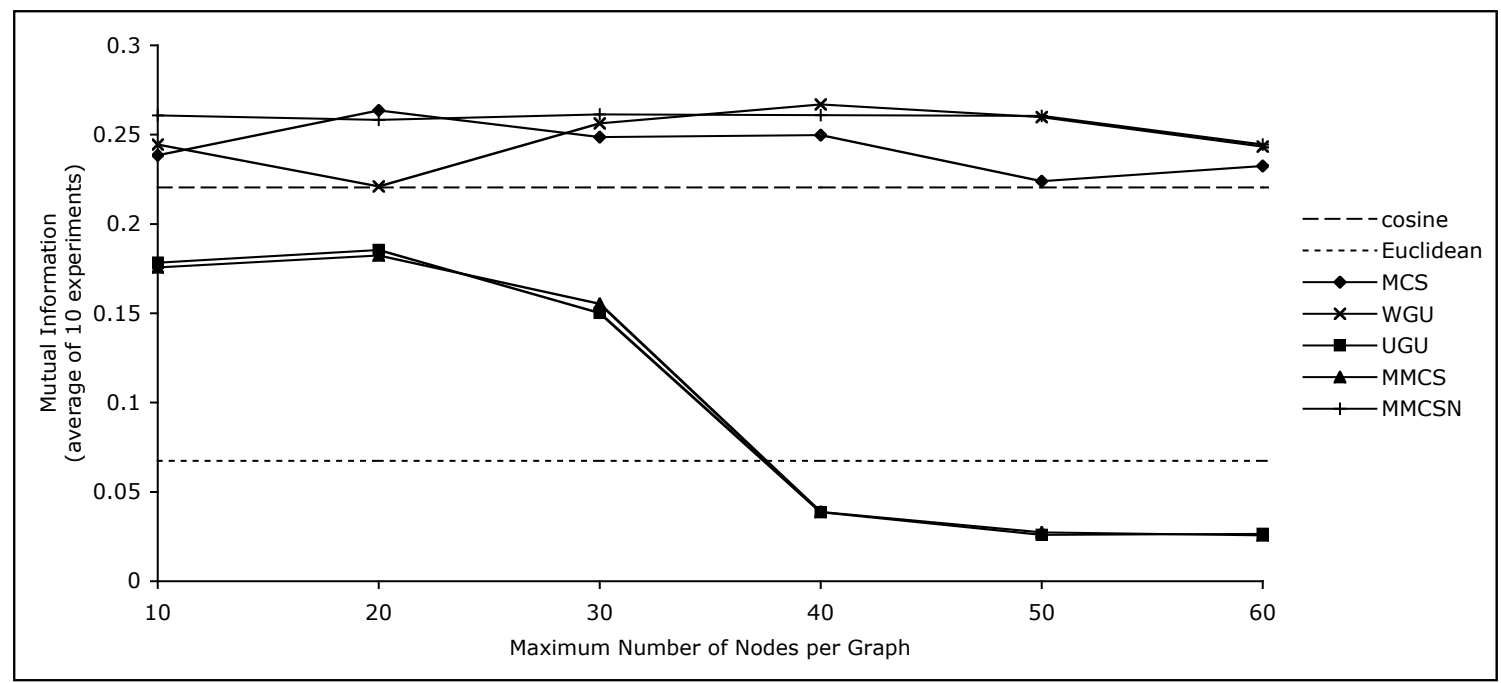

Figure 7.5. Distance Measure Comparison for the J-Series Data Set (Mutual Information)

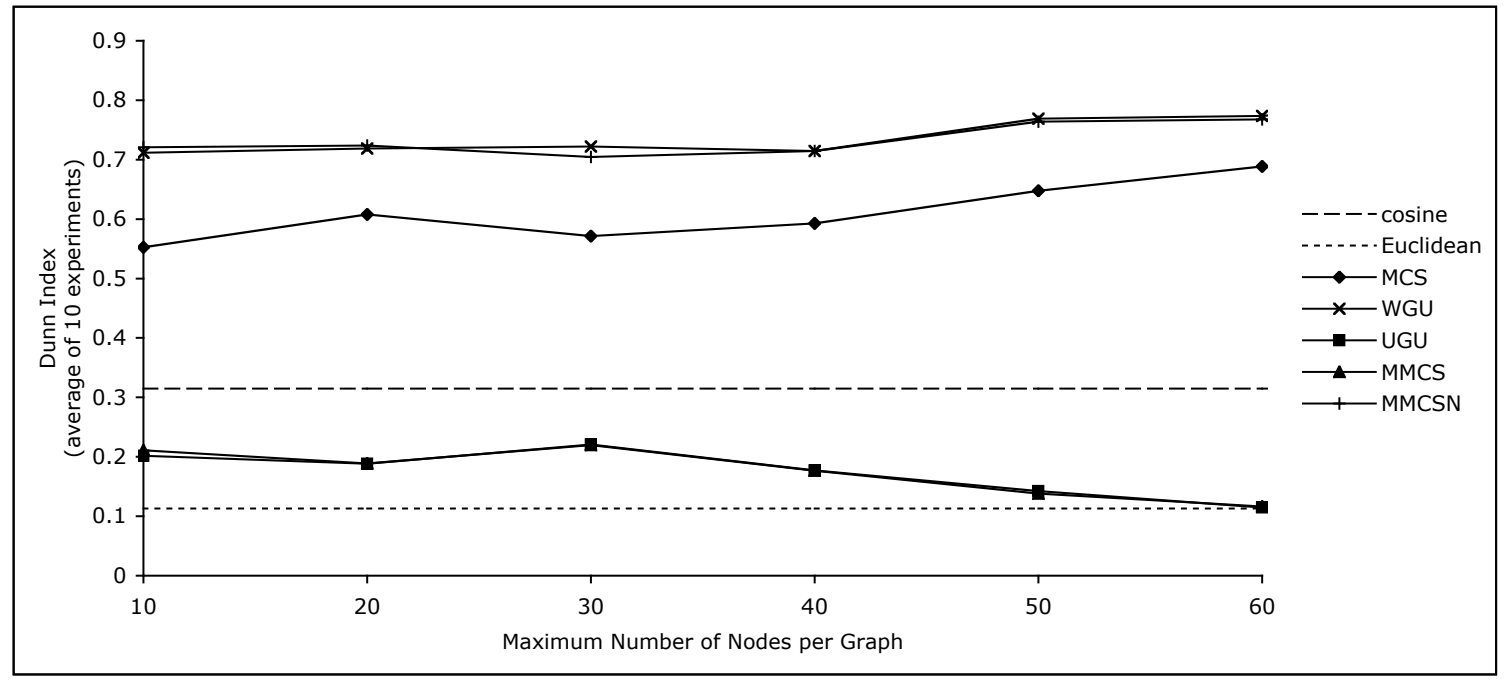

\section{Figure 7.6. Distance Measure Comparison for the J-Series data set (Dunn index)}

We see from the experimental results that the quality of clustering as measured by the Dunn index is more or less constant with the size of the graphs, with the distance measure seeming to have more effect than the data representation itself. We note similar behavior between mutual information and Rand index. For these two indices we see performance peaking at certain graph sizes but otherwise the performance is fairly constant. This could be due to the fact that the terms selected for those graph sizes provide particularly good discriminating information on the pre-defined set of categories. For larger graphs that indicate a decline in performance, it may be the case that we have polluted the graphs with unimportant terms which hurt the performance of the clustering algorithm. We also see that the results for each distance measure under all three 
performance indices are consistent across all three data sets. The fact that the graph-based methods performed as well or better than the vector-based method, even when the size of the graphs was relatively small, is encouraging and may indicate the quality of the added structural information that is captured in the graphs but not in the vector representations. Further, with the graph model there is also a potential for computational and space savings in terms of the data represented. For example, if we wish to add a new term to a document in a vector model representation, all documents must have their dimensionality increased by one (and thus the dimensionality of the feature space increases by one). However, if we want to add a new term to a document with a graph representation, we need only add the new node and edges to that particular graph, and the distance calculations between other graphs remains unaffected.

\section{Table 7.3. Graph Representation Comparison for K-Series}

\begin{tabular}{|c|c|c|c|}
\hline Representation & Rand Index & Mutual Information & Dunn Index \\
\hline standard & 0.8957 & 0.1174 & 0.0284 \\
\hline simple & 0.8870 & 0.0972 & 0.0274 \\
\hline 5-distance & 0.8813 & 0.1013 & 0.0206 \\
\hline 5-simple distance & 0.8663 & 0.0773 & 0.0234 \\
\hline absolute frequency & 0.8770 & 0.0957 & 0.0335 \\
\hline relative frequency & 0.8707 & 0.0992 & 0.0283 \\
\hline
\end{tabular}

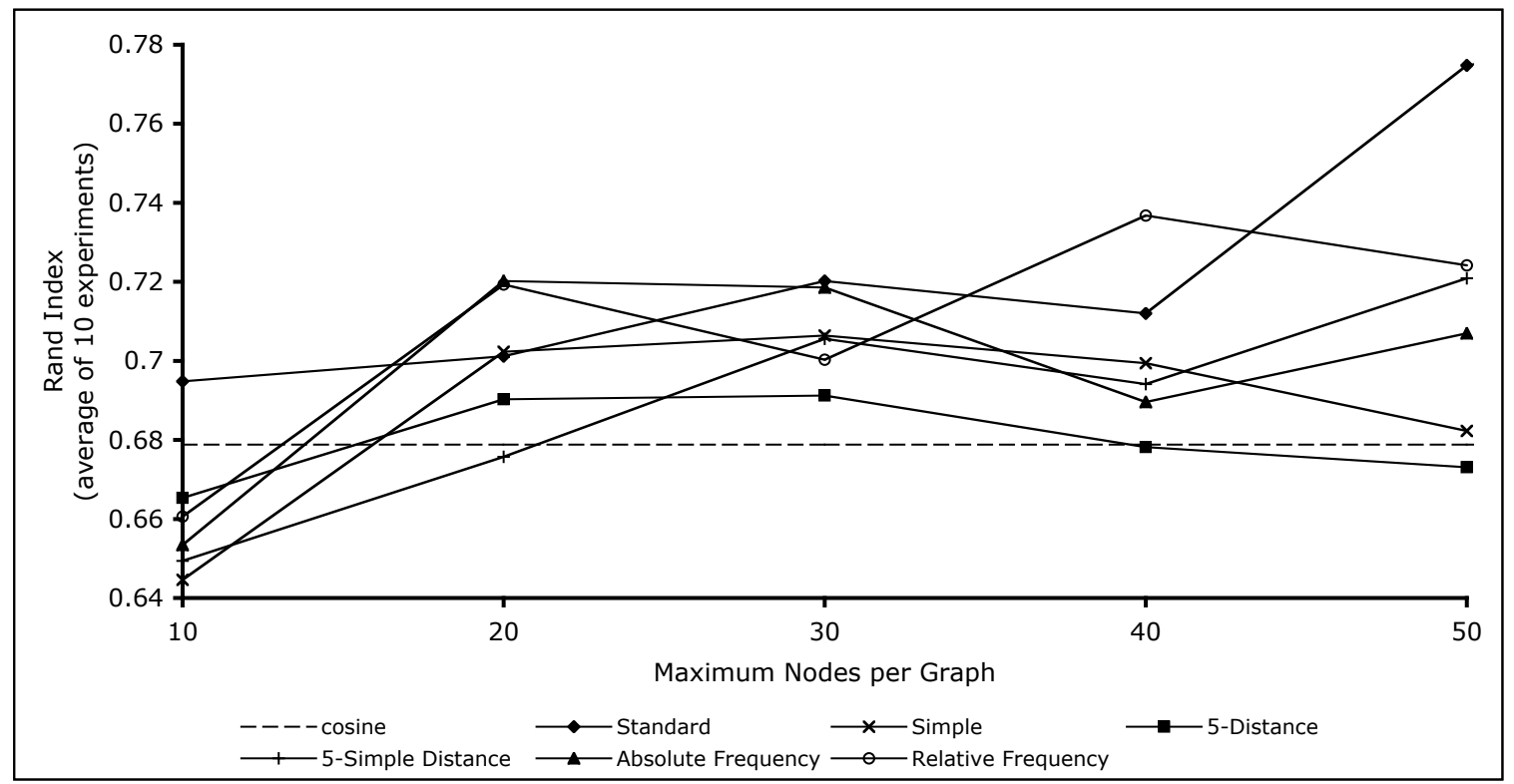

Figure 7.7. Graph Representation Comparison for the F-Series Data Set (Rand Index) 


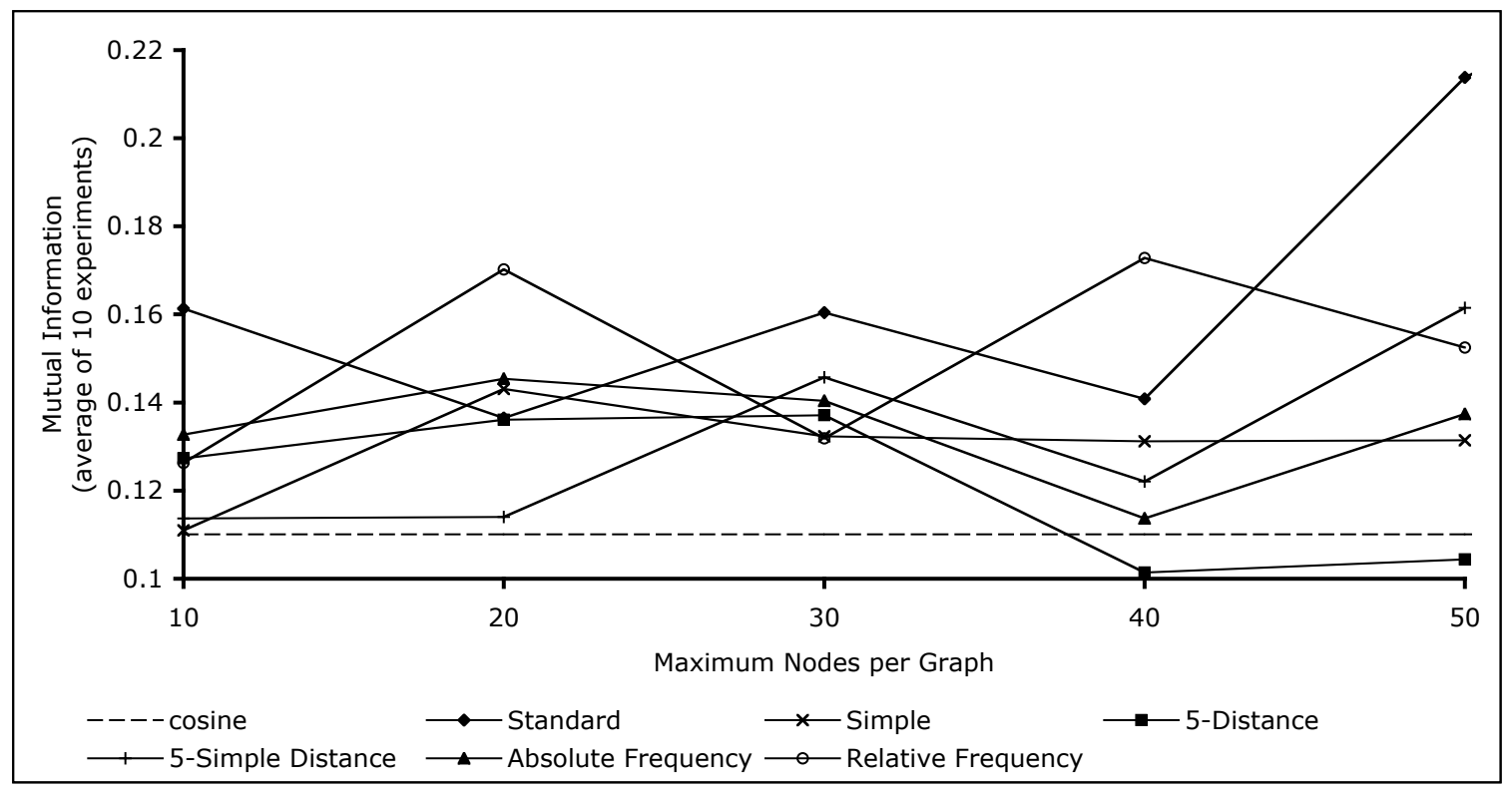

Figure 7.8. Graph Representation Comparison for the F-Series Data Set (Mutual Information)

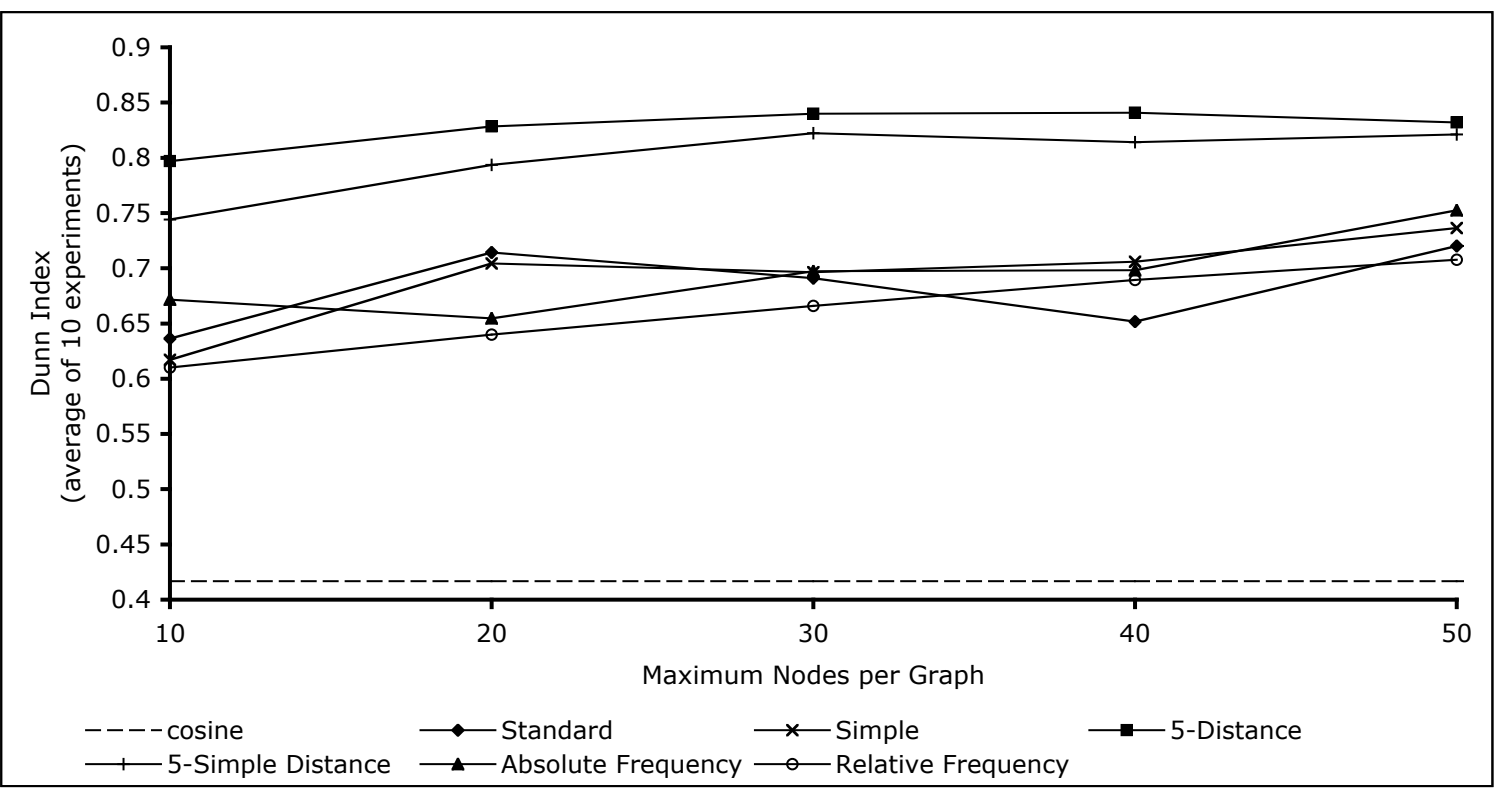

Figure 7.9. Graph Representation Comparison for the F-Series Data Set (Dunn Index) 


\subsection{Comparison of Graph Representations}

From our experimental results that compare our methods of representing web documents by graphs (Chapter 4), which are presented in Figs. 7.7-7.9 and 7.10-7.12 as a function of the maximum number of nodes allowed in a graph for the F-series and the $\mathrm{J}$ series, respectively, we see that the various graph representations can perform as well or better than the conventional vector model approach in terms of clustering accuracy (Rand and mutual information). The F-series showed good results for the graph representations, but the J-series was not quite as good, especially for representations other than standard and relative frequency. In terms of ranking the various graph representations, the results do not show a clear ordering that is consistent for both data sets with the exception that the standard and relative frequency representations are generally the best performing in terms of clustering accuracy (Rand and mutual information) and outperform the vector representation in most cases for both data sets. In terms of the Dunn index, which measures cluster compactness and separation, we obtain the best performance when using the $n$-distance or $n$-simple distance representations over the other graph representations and it is apparent for the Dunn index that 5-distance was the best performing with 5simple distance a close second. The effect of the maximum graph size (number of nodes) on the clustering performance is not obvious. Consider the standard representation. For the $\mathrm{J}$-series the accuracy decreases at 30 nodes after reaching a maximum value at 20 nodes and there is another drop between 40 and 50 nodes, while in the F-series we see the opposite behavior for those graph sizes. On the other hand, the quality of clustering itself (Dunn index) does not seem to be sensitive at all to the number of nodes.

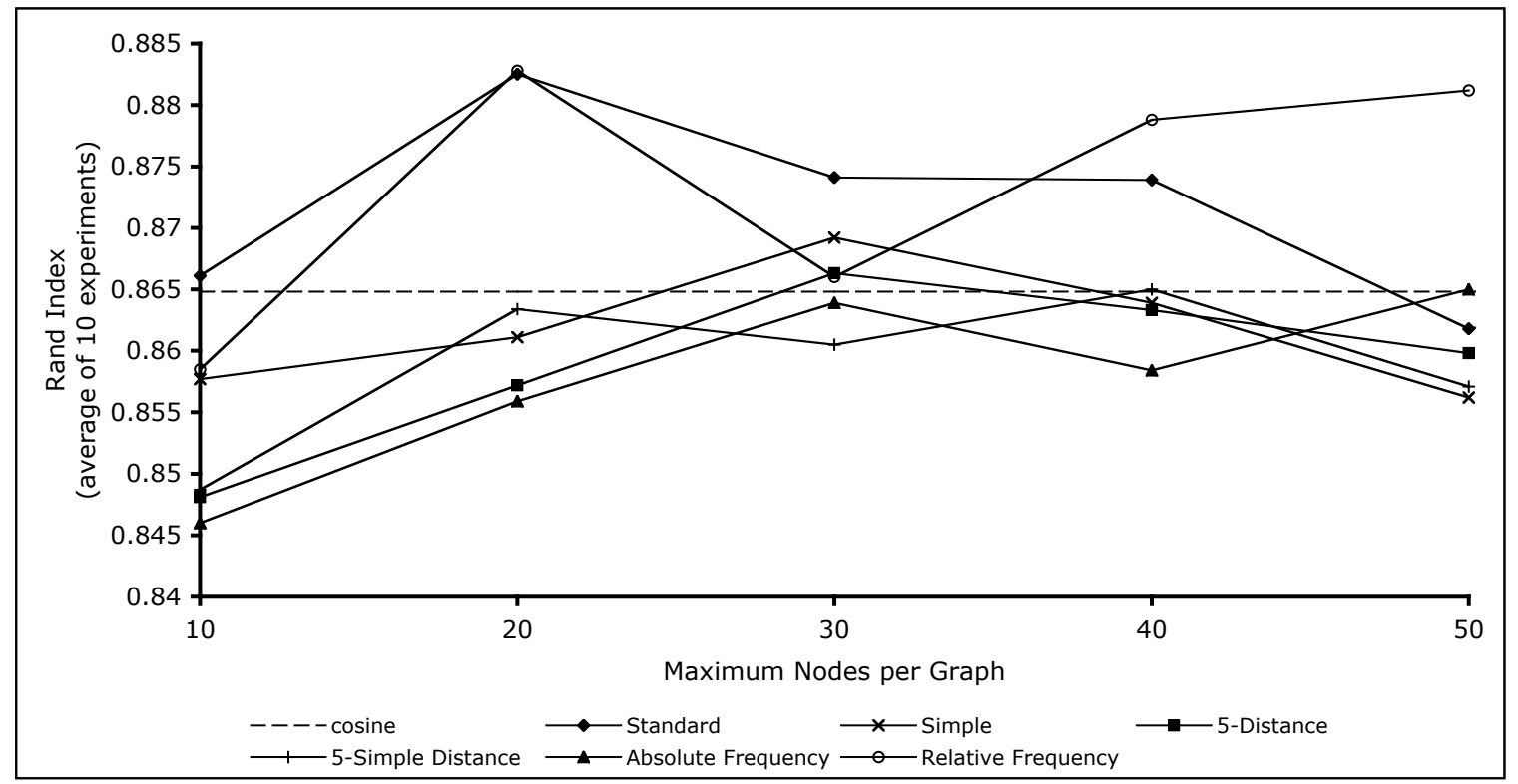

Figure 7.10. Graph Representation Comparison for the J-Series Data Set (Rand Index) 


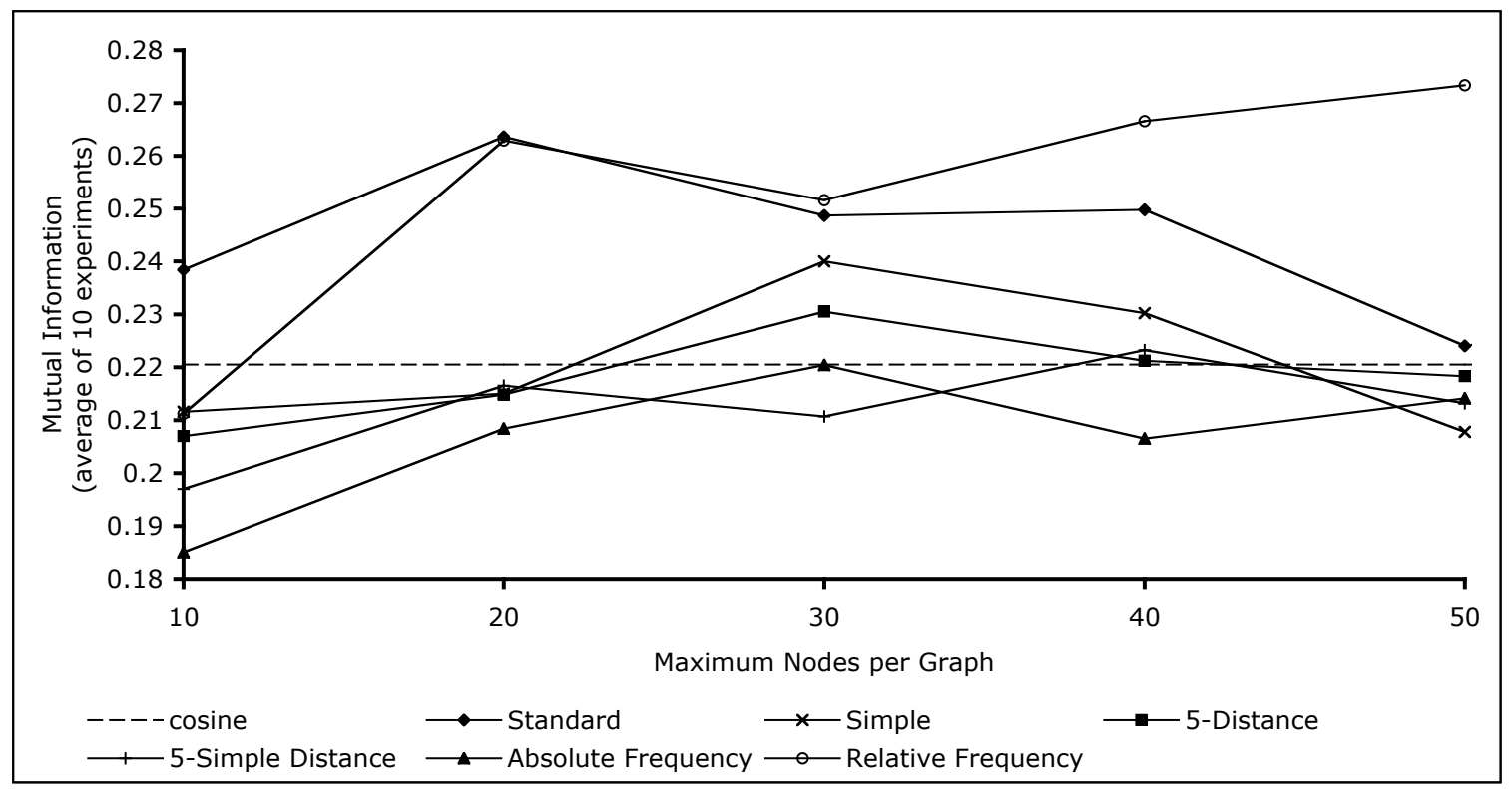

Figure 7.11. Graph Representation Comparison for the J-Series Data Set (Mutual Information)

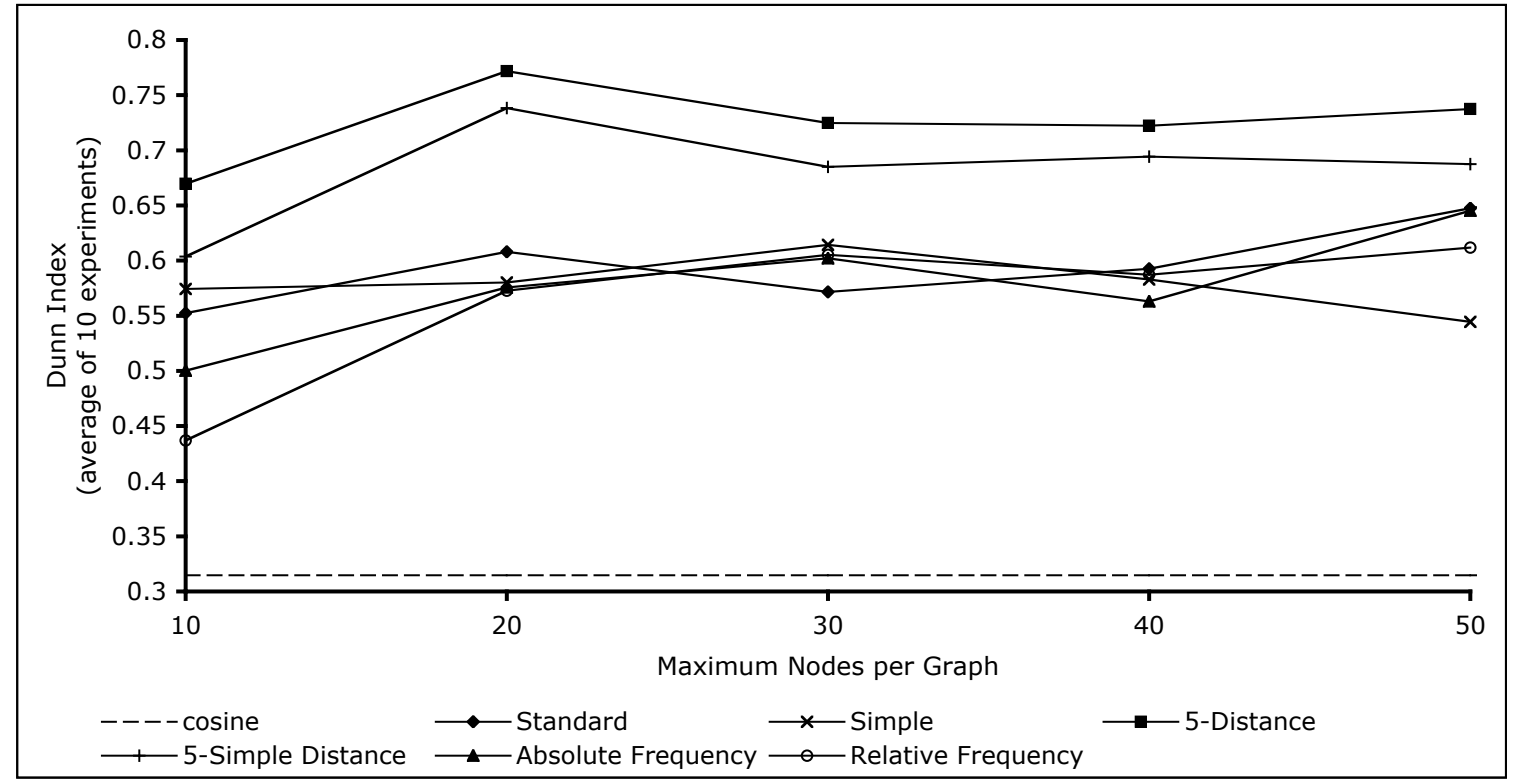

Figure 7.12. Graph Representation Comparison for the J-Series Data Set (Dunn Index)

The results for clustering the K-series data set with the various graph representations while using 70 nodes per graph maximum are given in Table 7.3. The results show that the standard representation was the best performing in terms of both 
Rand index and mutual information, while absolute frequency provided the best Dunn index score.

The experiments in this chapter relied on a random initialization of the $k$-means algorithm, which is the conventional way the algorithm is applied. However, using a random initialization has drawbacks. First, the initialization may be poor and lead the algorithm to converge on a bad final clustering of the data. Second, the fact that each clustering created using the same algorithm may be different leads to difficulty in comparing experimental results. The usual methodology is to perform a series of experiments and report the average, as was done here. However, this is time consuming as the same experiment must be performed repeatedly. Recently a new method of deterministically arriving at a good initialization state for the $k$-means algorithm has been reported. In the next chapter we will create a graph-theoretical version of this method and apply it to our web data sets for the purpose of examining the effect it has on clustering performance.
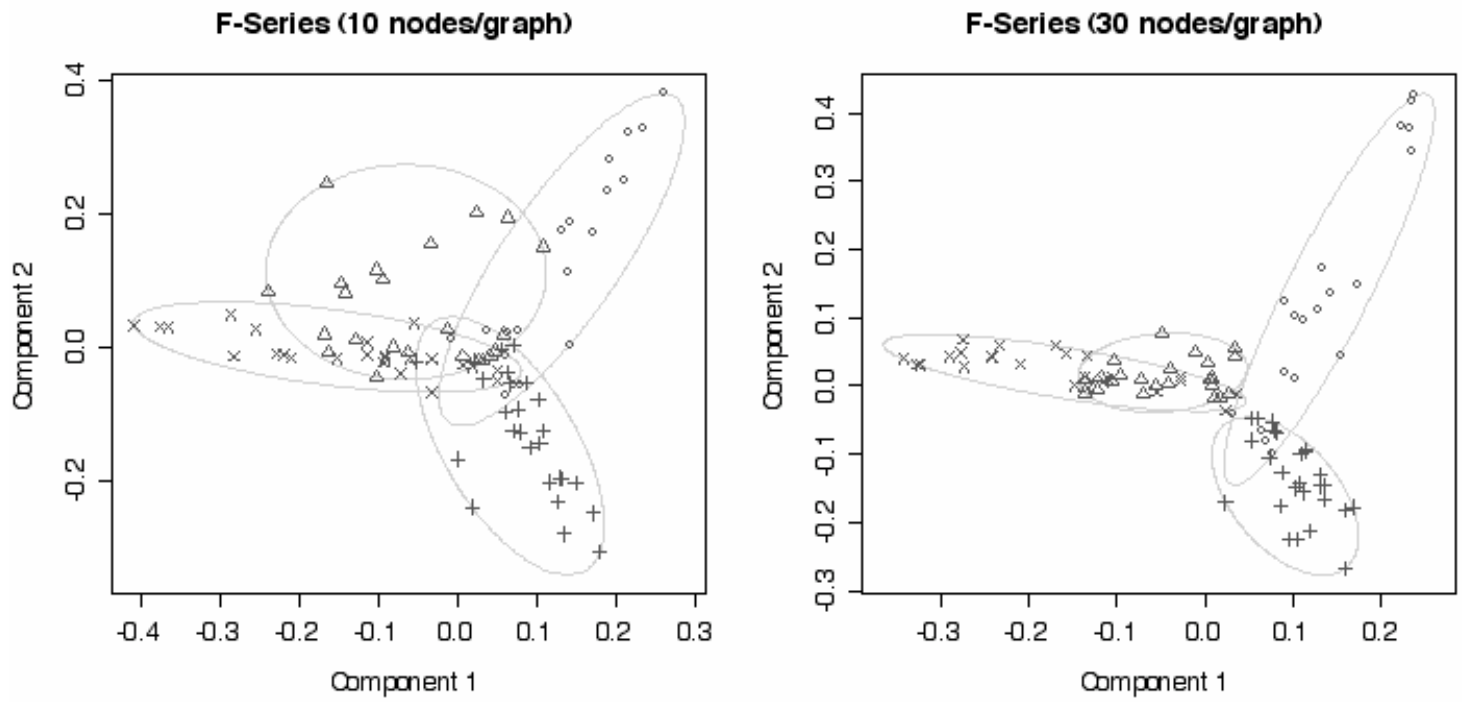

Figure 7.13. F-Series Data Set Represented by Graphs Scaled to Two Dimensions Using 10 Nodes/Graph (Left) and 30 Nodes/Graph (Right)

\subsection{Visualization of Graph Clustering}

Multidimensional scaling [24] is a mathematical technique for transforming spaces that are complex or not well understood into a lower dimensional Euclidean feature space. The procedure attempts to preserve the original distances between objects in the Euclidean representation. This method is useful for visualizing object relationships that are not easily represented graphically, such as the relationships between the graphs that are used to represent web documents in our experiments. By representing web documents (or other complex entities) as graphs and then taking the pair-wise distance between the graphs using one of the graph-theoretical distance measures we can apply

multidimensional scaling to arrive at a representation of the graphs in $\mathfrak{R}^{2}$. This allows the 
graphs to be plotted graphically as points on an $x-y$ plane, which is not possible for the original untransformed space.
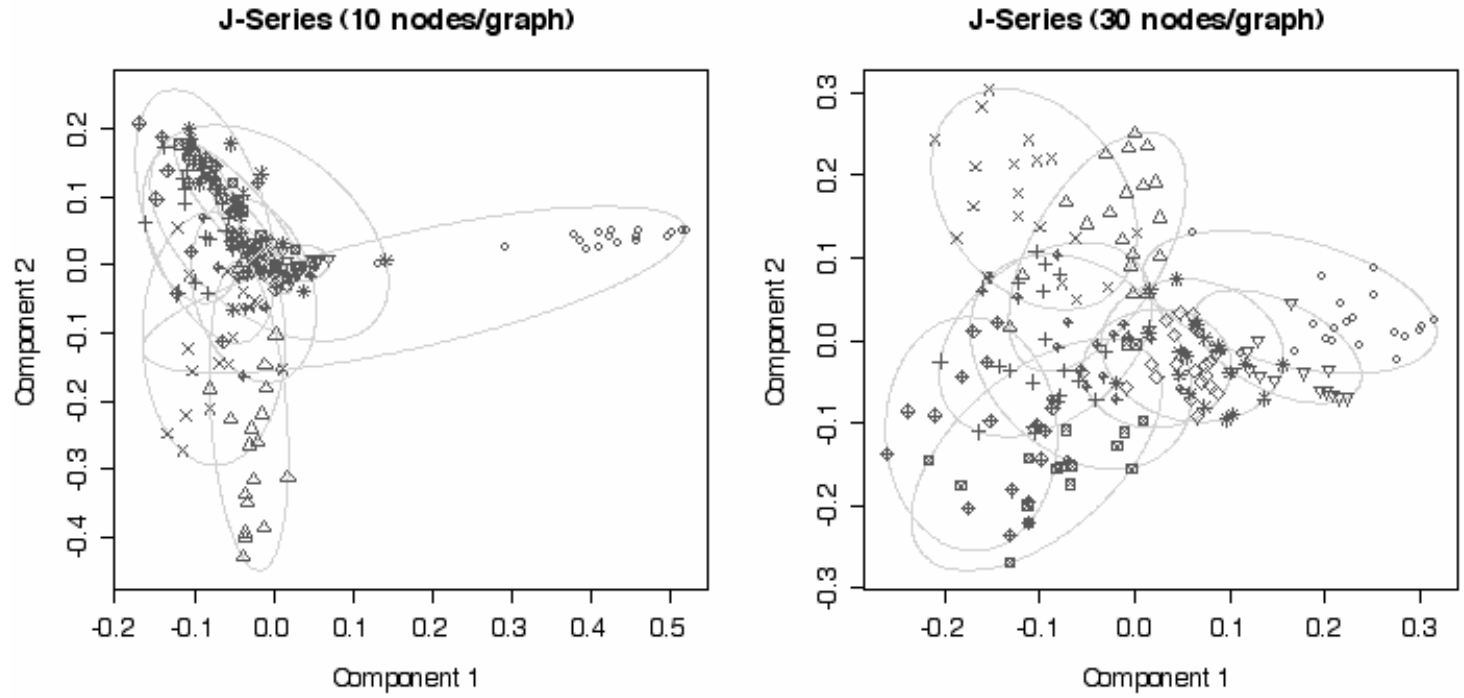

Figure 7.14. J-Series Data Set Represented by Graphs Scaled to Two Dimensions Using 10 Nodes/Graph (Left) and 30 Nodes/Graph (Right)
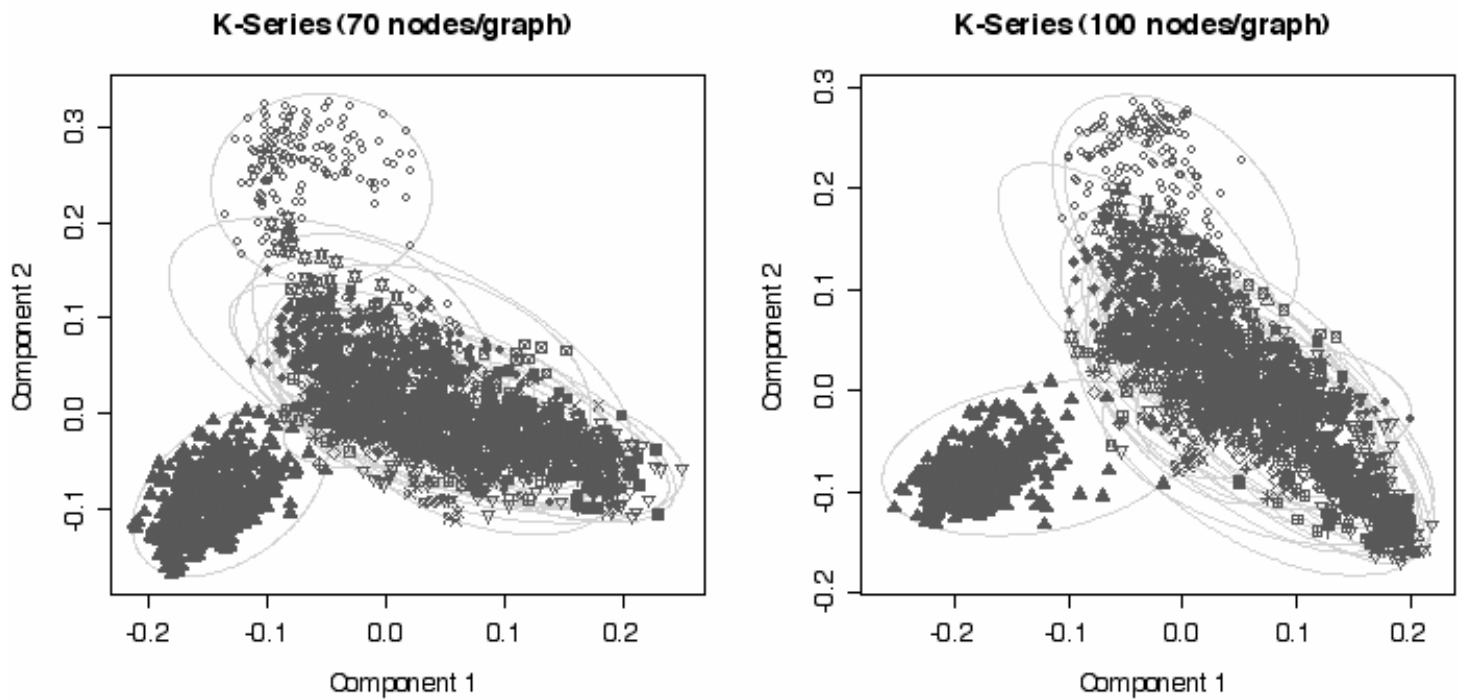

Figure 7.15. K-Series Data Set Represented by Graphs Scaled to Two Dimensions Using 70 Nodes/Graph (Left) and 100 Nodes/Graph (Right)

We have performed multidimensional scaling on our data sets using the standard representation and MCS distance to create graphs from the web pages. The results of the scaling are shown in Fig. 7.13 for the F-series, Fig. 7.14 for the J-series, and Fig 7.15 for 
the $\mathrm{K}$-series. For the $\mathrm{F}$ and $\mathrm{J}$ series, the left side shows the results when using 10 nodes per graph; the right side utilizes 30 nodes per graph. For the K-series the left side is 70 nodes per graph while the right is 100 nodes per graph. Each graph is plotted as a point in a two-dimensional Euclidean space; the symbol of each point is given by the ground truth cluster the graph belongs to. The ellipses give an indication of cluster shape and size (they are fit to cover all the points of each cluster while minimizing the area).

The F-series plot clearly shows the four clusters of this data set. Though there is some overlap towards the center of the plot, many of the data points can be easily differentiated. Looking at the change between the left and right plots, we see cluster compactness and separation improves as we add more nodes to the graphs. For example, the bottom right cluster (pluses) is smaller and no longer overlaps the center cluster (triangles) or middle left cluster (crosses). Similarly, the top right cluster (circles) is also more compact and no longer overlaps the middle or middle left clusters. This is an intuitively appealing result. The J-series is more difficult to view since it contains many overlapping clusters. However, we see the data points become much more evenly distributed as we increase the graph size (on the left plot many of the points are concentrated in the top left area). This allows for easier differentiation between clusters. We should note that with multidimensional scaling there is some information loss due to the reduction of the number of dimensions; thus the plots here will not correspond exactly to actual clustering performance as performed in the graph domain. However, the apparent increase in cluster compactness and separation with larger graph sizes seems reasonable. The $\mathrm{K}$-series contains more than ten times the number of documents of the Jseries, making it more difficult to view individual web documents. However, two wellseparated clusters are clearly visible on the figures: the bottom left cluster (the triangles) and the top middle cluster (circles). The effect of increasing the number of nodes per graph is less dramatic than in the other two data sets. This is probably due either to the amount of information added by going from 70 to 100 nodes per graph being less than the increase in information when going from 10 to 30 in the other data sets, or that this data set is simply to complex (too large, too many clusters) to visualize well. 


\section{Chapter Eight}

\section{The Graph-Theoretic Global k-Means Algorithm}

\subsection{Introduction}

In our previous experiments with clustering we used random initializations at the first step of the $k$-means algorithm. Recently Likas et al. [71] have introduced what they call the global k-means method. This method provides a way of determining "good" initial cluster centers for the $k$-means algorithm, without having to use random initializations. Their experimental results have shown clustering performance under global $k$-means which is as good or better than using random initializations. The basic procedure is an incremental computation of cluster centers. Starting at the case of one cluster $(k=1)$, the cluster center is defined to be the centroid of the entire data set. For the general case of $k$ clusters, the centers are determined by taking the centers from the $k$ 1 clusters problem and then determining the optimum location of a new center. This is accomplished by considering each data item as the new cluster center and then executing the $k$-means algorithm with that particular set of cluster centers and determining which data point minimizes the error as defined by:

$$
E\left(m_{1}, \ldots, m_{M}\right)=\sum_{i=1}^{N} \sum_{k=1}^{M} I\left(x_{i} \in C_{k}\right)\left\|x_{i}-m_{k}\right\|^{2}
$$

where $N$ is the number of data items, $M$ is the number of clusters, $x_{i}$ is data item $i, m_{k}$ is cluster center $k$, and $I(X)=1$ if $X$ is true and 0 otherwise. A problem with this approach is that it requires execution of the $k$-means algorithm $O(N \cdot M)$ times. For many applications this will be too time-consuming. With this in mind, the authors have also proposed a "fast" version of global $k$-means. Under this method, instead of running $k$-means when considering each data item as a new cluster center candidate, we calculate the following:

$$
b_{n}=\sum_{j=1}^{N} \max \left(d_{k-1}^{j}-\left\|x_{n}-x_{j}\right\|^{2}, 0\right)
$$

where $d_{k-1}^{j}$ is the distance between data item $x_{j}$ and its closest cluster center for the $k-1$ clustering problem. We then select the new cluster center to be data item $x_{i}$ where:

$$
i=\arg \max _{n} b_{n}
$$


It is this "fast" version of the global $k$-means method that we have implemented and that we will use for our experiments in this chapter. Specifically we will apply the global $k$ means method to our graph-based $k$-means algorithm to see if there is any improvement over the previous cases that used random initializations [117]. Further, due to the incremental nature of the global $k$-means method, where determining the initial centers for $k$ clusters implies we must also determine the initial centers for 1 to $k-1$ clusters, this provides an opportunity to examine the question of automatically determining the optimum number of clusters. Many clustering methods, including $k$-means, require the user to specify in advance the number of clusters to create. With a method that automatically determines the number of clusters we do not have to do this, which is beneficial if the number of clusters is not known a priori. In Section 8.2 we will give experimental results which compare clustering performance under global $k$-means as compared with random initialization. In Section 8.3 we will look at the problem of automatically determining the best number of clusters; we will run both the global $k$ means and random methods for a range of $k$ values and calculate various performance indices for each case.

Cluster validity has long been studied and several approaches are represented in the literature. In [41], Frigui and Krishnapuram present a clustering algorithm based on fuzzy $c$-means which determines the optimal number of clusters by merging similar clusters, thus eliminating unimportant or spurious clusters. In [64][94] interesting methods of finding the number of clusters with a procedure based on scale-space persistence are presented. In general terms, an ever expanding neighborhood is examined. When the neighborhood is at a minimum, every data point is in its own separate cluster. As the neighborhood expands clusters are merged until eventually there is one giant cluster comprising the entire data set. This can be seen as "zooming" in or out on the data, effectively looking at different levels of granularity. By performing this zooming over a range of fixed increments, we can look at how many clusters exist at each increment. The optimum number of clusters is then the one that persisted over the largest range of increments. Hardy investigates seven methods of determining the number of clusters in [52]. One of his observations is that since every clustering method has an underlying implicit cluster characteristic that it prefers, we must be sure to choose an algorithm that matches the structure of the data. Cluster validity using graph-theoretic concepts in place of traditional validity indices was investigated in [98]. New cluster validity methods for the fuzzy $c$-means algorithm have been proposed in [104][137]. New clustering algorithms which explicitly take into account cluster validity are given in [135][136].

\subsection{Global $k$-Means vs. Random Initialization}

We performed a series of experiments on the F-series and J-series data sets; the results are presented in Tables 8.1 to 8.4 for values of $k$ equal to the number of clusters present in ground truth ( $k=4$ for F-series, $k=10$ for J-series). We compared our usual graph-based method using MCS distance and the standard representation to the vectorbased method using distance based on Jaccard similarity. The results show that in all cases, whether graph or vector related, the global $k$-means method outperformed the 
corresponding random method. Here random denotes the average of ten experiments each using a random initialization. This reaffirms the original experimental results of [71]. The results also show that, except for the case of F-series data set when using 10 nodes per graph, our graph-based method outperformed the vector method. The execution times for the experiments are also given in Tables 8.5 and 8.6. All experiments were carried out on the same system under the same operating conditions: an un-loaded $296 \mathrm{MHz}$ Sun UltraSPARC-II with 1,024 megabytes of memory. As expected, the execution time for global $k$-means is much greater than random, due to the need to compute the cluster centers. We also see the potential for a time savings over the vector case when using small graphs. For the J-series, not only was using the graph-based method with a maximum graph size of 10 nodes better performing than the vector case, it was faster by nearly four and a half hours.

Table 8.1. Results for F-Series (Rand Index)

\begin{tabular}{|c|c|c|c|c|}
\cline { 2 - 5 } \multicolumn{1}{c|}{} & \multicolumn{2}{c|}{ Global k-means } & \multicolumn{2}{c|}{ Random } \\
\hline Graph Size & Jaccard & Graphs & Jaccard & Graphs \\
\hline 10 & 0.7057 & 0.7281 & 0.6899 & 0.6730 \\
\hline 20 & 0.7057 & 0.7976 & 0.6899 & 0.7192 \\
\hline 30 & 0.7057 & 0.7838 & 0.6899 & 0.7394 \\
\hline
\end{tabular}

Table 8.2. Results for F-Series (Mutual Information)

\begin{tabular}{|c|c|c|c|c|}
\cline { 2 - 5 } \multicolumn{1}{c|}{} & \multicolumn{2}{c|}{ Global k-means } & \multicolumn{2}{c|}{ Random } \\
\hline Graph Size & Jaccard & Graphs & Jaccard & Graphs \\
\hline 10 & 0.1914 & 0.1653 & 0.102 & 0.1498 \\
\hline 20 & 0.1914 & 0.2274 & 0.102 & 0.1638 \\
\hline 30 & 0.1914 & 0.2336 & 0.102 & 0.1793 \\
\hline
\end{tabular}

Table 8.3. Results for J-Series (Rand Index)

\begin{tabular}{|c|c|c|c|c|}
\cline { 2 - 5 } \multicolumn{1}{c|}{} & \multicolumn{2}{c|}{ Global k-means } & \multicolumn{2}{c|}{ Random } \\
\hline Graph Size & Jaccard & Graphs & Jaccard & Graphs \\
\hline 10 & 0.8809 & 0.9049 & 0.8717 & 0.8689 \\
\hline 20 & 0.8809 & 0.9065 & 0.8717 & 0.8819 \\
\hline 30 & 0.8809 & 0.9056 & 0.8717 & 0.8758 \\
\hline
\end{tabular}


Table 8.4. Results for J-Series (Mutual Information)

\begin{tabular}{|c|c|c|c|c|}
\cline { 2 - 5 } \multicolumn{1}{c|}{} & \multicolumn{2}{c|}{ Global k-means } & \multicolumn{2}{c|}{ Random } \\
\hline Graph Size & Jaccard & Graphs & Jaccard & Graphs \\
\hline 10 & 0.2787 & 0.3048 & 0.2316 & 0.2393 \\
\hline 20 & 0.2787 & 0.3135 & 0.2316 & 0.2597 \\
\hline 30 & 0.2787 & 0.3188 & 0.2316 & 0.2447 \\
\hline
\end{tabular}

Table 8.5. Execution Times Using Random Initialization (in Seconds)

\begin{tabular}{|l|c|c|c|c|}
\hline \multicolumn{5}{|c|}{ Random (average of 10 experiments) } \\
\hline & Graphs -10 & Graphs -20 & Graphs - 30 & Jaccard \\
\hline F-series & 84.4 & 126.1 & 205.3 & 24.5 \\
\hline J-series & 173.1 & 396.4 & 550.2 & 214.9 \\
\hline
\end{tabular}

Table 8.6. Execution Times Using Global $k$-Means (in Minutes)

\begin{tabular}{|l|c|c|c|c|}
\hline \multicolumn{5}{|c|}{ Global $k$-means } \\
\hline & Graphs -10 & Graphs -20 & Graphs -30 & Jaccard \\
\hline F-series & 11.87 & 24.88 & 38.68 & 14.57 \\
\hline J-series & 239.55 & 545.92 & 818.47 & 507.55 \\
\hline
\end{tabular}

\subsection{Optimum Number of Clusters}

Previously we have used three indices to measure clustering performance: Rand index, mutual information, and Dunn index. Both Rand and mutual information compare the clustering produced by an algorithm to the actual ground truth clustering. For the problem of determining the optimum number of clusters automatically, we cannot assume that a ground truth clustering is available for evaluation. In this case we need a cluster validation index, which is a measure of clustering quality that is not dependant on knowing the ground truth clustering. The Dunn index (Eq. 6.3) is one such index, however it is sensitive to noise and outliers. Some other notable indices have been reported in the literature, and we present some of them here. First we have the $C$ index [56], which is defined as:

$$
C=\frac{S-S_{\min }}{S_{\max }-S_{\min }}
$$

where $S$ is the sum of all distances of pairs of items in the same cluster. We define $l$ to be the number of these pairs used to compute $S . S_{\min }$ and $S_{\max }$ are the sum of the $l$ smallest and largest distances, respectively. The smaller the value of $C$, the better the clustering.

Another validity index is the Davies-Bouldin index [30], defined as: 


$$
D B=\frac{1}{M} \sum_{i=1}^{M} \max _{j=1, \ldots, M ; j \neq i}\left(d_{i j}\right)
$$

where $M$ is the number of clusters and

$$
d_{i j}=\frac{r_{i}+r_{j}}{d\left(c_{i}, c_{j}\right)}
$$

Here $r_{i}$ is the average distance of all data items in cluster $i$ to their cluster center and $d\left(c_{i}, c_{j}\right)$ is the distance between the centers of clusters $i$ and $j . d_{i j}$ measures, similar to the Dunn index, the compactness (numerator) and separation (denominator) of cluster pairs. A small value of the Davies-Bouldin index is desirable.

Finally, we have the Goodman-Kruskal index [44], which is somewhat similar to the Rand index. In the Goodman-Kruskal method we examine all quadruples of data items $(q, r, s, t)$ and look to see if they conform to one of the following cases:

(1) $d(q, r)<d(s, t) ; q$ and $r$ in the same cluster; $s$ and $t$ in different clusters

(2) $d(q, r)>d(s, t) ; q$ and $r$ in different clusters; $s$ and $t$ in the same cluster

(3) $d(q, r)<d(s, t) ; q$ and $r$ in different clusters; $s$ and $t$ in the same cluster

(4) $d(q, r)>d(s, t) ; q$ and $r$ in the same cluster; $s$ and $t$ in different clusters

If we have case (1) or case (2), this is called concordant and indicates that pairs of items that are in the same cluster should have a smaller distance than pairs of items that are in different clusters. Similarly, cases (3) and (4) are called discordant. Let $S^{+}$be the number of concordant quadruples and $S^{-}$be the number of discordant quadruples. The GoodmanKruskal index is then given by

$$
G K=\frac{S^{+}-S^{-}}{S^{+}+S^{-}}
$$

A large value for $G K$ indicates a good clustering (i.e. high concordance). A problem with this method is immediately evident, however: the complexity of computing $G K$ is $O\left(n^{4}\right)$, where $n$ is the number of items in the data set. Thus computing this index can be more time consuming than performing the clustering itself.

We performed experiments for values of $k$ varying from 2 to 10 for both the Fseries and the J-series data sets when using both global $k$-means or a random initialization. Here random initialization is accomplished as before by randomly assigning each data item to a cluster. Note that it is not possible to re-use the same random initialization for different values of $k$, thus each experiment has a separate random initialization. Our graphs were created using the standard representation and a maximum of 10 nodes/graph; the distance measure used was MCS. The results are presented in Tables 8.7 to 8.10. The "best" number of clusters is determined from Rand 
index and mutual information, which indicate the performance as compared to ground truth.

Table 8.7. Results for F-Series Using Global $k$-Means

\begin{tabular}{|c|c|c|c|c|c|c|}
\hline $\begin{array}{c}\text { \# of } \\
\text { clusters }\end{array}$ & Dunn & Davies-Bouldin & Goodman-Kruskal & C Index & Rand & $\begin{array}{c}\text { Mutual } \\
\text { Information }\end{array}$ \\
\hline 2 & 0.6667 & 1.8567 & 0.3431 & 0.2451 & 0.5304 & 0.0978 \\
\hline 3 & 0.6667 & 1.8665 & 0.5163 & 0.3687 & 0.6604 & 0.1231 \\
\hline 4 & 0.6667 & 1.7833 & 0.6161 & 0.4188 & 0.7281 & 0.1653 \\
\hline 5 & 0.6667 & 1.7785 & 0.6795 & 0.4391 & 0.7578 & 0.1868 \\
\hline 6 & 0.6667 & 1.7091 & 0.7207 & 0.4028 & 0.7665 & 0.2156 \\
\hline 7 & 0.6667 & 1.6713 & 0.7588 & 0.3745 & 0.7775 & 0.2186 \\
\hline 8 & 0.6667 & 1.7688 & 0.7557 & 0.3780 & 0.7695 & 0.2090 \\
\hline 9 & 0.6667 & 1.6971 & 0.7956 & 0.3385 & 0.7761 & 0.2205 \\
\hline 10 & 0.6667 & 1.6560 & 0.8109 & 0.3236 & 0.7779 & 0.2229 \\
\hline
\end{tabular}

We see from the results that these two indices agreed on (i.e., were optimal for) the same value for $k$ for the experiments that used global $k$-means. For the experiments using random initialization, there was not an agreement and consequently we can't decide definitively on the "best" number of clusters in these cases. The next observation regarding the results is that both Davies-Bouldin and Goodman-Kruskal always agreed on the same $k$ value. Further, the agreement of these two performance indices also coincides with the optimal values for Rand index and mutual information determined using global $k$-means. Dunn and $\mathrm{C}$ index do not seem very useful in terms of finding the correct $k$ value. We see that only in the case of global $k$-means with the F-series did Dunn agree with the other indices, and even then the Dunn index had identical values for all $k$. $\mathrm{C}$ index fared slightly better, agreeing with the other indices for global $k$-means for the $\mathrm{J}$ series; although the optimal value was at $k=2$ for global $k$-means with the F-series, its second best value was at the correct $k$ value. We note that global $k$-means was clearly much better in terms of agreement between cluster performance indices than random: for both $\mathrm{F}$ and $\mathrm{J}$-series five out of six indices agreed for global $k$-means, whereas random only achieved three out of six agreements (F-series) or two out of six agreements ( $\mathrm{J}$ series). Finally, we see that for the F-series, a larger number of clusters is indicated than is present in ground truth. This could be due to the fact that the F-series data set originally contained many more classes (see Section 6.3). Recall that we altered the ground truth to four larger clusters which subsumed the more specific topics. It is possible that this structure is still apparent in the data, even though we are using only a maximum of 10 terms per graph. Another possibility is that the indices we are using are sometimes skewed towards a larger number of clusters. For example, when the number of clusters equals the number of data items (i.e. each data item is its own cluster), indices that measure clustering performance as a function of the distance of data items from their cluster centers become maximized. 
Table 8.8. Results for F-Series Using Random Initializations

\begin{tabular}{|c|c|c|c|c|c|c|}
\hline $\begin{array}{c}\text { \# of } \\
\text { clusters }\end{array}$ & Dunn & Davies-Bouldin & Goodman-Kruskal & C Index & Rand & $\begin{array}{c}\text { Mutual } \\
\text { Information }\end{array}$ \\
\hline 2 & 0.6667 & 1.8567 & 0.3431 & 0.2451 & 0.5304 & 0.0978 \\
\hline 3 & 0.6667 & 1.8660 & 0.5440 & 0.3480 & 0.6676 & 0.1317 \\
\hline 4 & 0.6667 & 1.7983 & 0.6202 & 0.4173 & 0.7169 & 0.1530 \\
\hline 5 & 0.6667 & 1.9934 & 0.5629 & 0.4889 & 0.7057 & 0.1423 \\
\hline 6 & 0.6818 & 1.7981 & 0.6980 & 0.4244 & 0.7644 & 0.1844 \\
\hline 7 & 0.6667 & 1.8774 & 0.6772 & 0.4545 & 0.7634 & 0.2017 \\
\hline 8 & 0.6471 & 1.9110 & 0.6615 & 0.4763 & 0.7695 & 0.2160 \\
\hline 9 & 0.6667 & 1.6304 & 0.7472 & 0.4011 & 0.7831 & 0.2154 \\
\hline 10 & 0.6667 & 1.7314 & 0.7086 & 0.4610 & 0.7751 & 0.2127 \\
\hline
\end{tabular}

Table 8.9. Results for J-Series Using Global $k$-Means

\begin{tabular}{|c|c|c|c|c|c|c|}
\hline $\begin{array}{c}\text { \# of } \\
\text { clusters }\end{array}$ & Dunn & Davies-Bouldin & Goodman-Kruskal & C Index & Rand & $\begin{array}{c}\text { Mutual } \\
\text { Information }\end{array}$ \\
\hline 2 & 0.4286 & 1.9427 & 0.2376 & 0.2950 & 0.4850 & 0.0911 \\
\hline 3 & 0.6250 & 1.8845 & 0.4544 & 0.3987 & 0.6830 & 0.1435 \\
\hline 4 & 0.6500 & 1.8174 & 0.5328 & 0.4338 & 0.7618 & 0.1848 \\
\hline 5 & 0.6000 & 1.7792 & 0.5797 & 0.4122 & 0.7986 & 0.2041 \\
\hline 6 & 0.6000 & 1.7768 & 0.6612 & 0.3610 & 0.8471 & 0.2480 \\
\hline 7 & 0.6000 & 1.7653 & 0.6692 & 0.3612 & 0.8638 & 0.2599 \\
\hline 8 & 0.6154 & 1.7453 & 0.7300 & 0.3177 & 0.8833 & 0.2819 \\
\hline 9 & 0.6154 & 1.7612 & 0.7543 & 0.2947 & 0.8978 & 0.2978 \\
\hline 10 & 0.6154 & 1.7379 & 0.7686 & 0.2855 & 0.9049 & 0.3048 \\
\hline
\end{tabular}

Table 8.10. Results for J-Series Using Random Initializations

\begin{tabular}{|c|c|c|c|c|c|c|}
\hline $\begin{array}{c}\text { \# of } \\
\text { clusters }\end{array}$ & Dunn & Davies-Bouldin & Goodman-Kruskal & C Index & Rand & $\begin{array}{c}\text { Mutual } \\
\text { Information }\end{array}$ \\
\hline 2 & 0.4286 & 1.9427 & 0.2376 & 0.2950 & 0.4850 & 0.0911 \\
\hline 3 & 0.4286 & 1.9337 & 0.1970 & 0.4466 & 0.5818 & 0.0724 \\
\hline 4 & 0.5833 & 1.8693 & 0.3849 & 0.4830 & 0.7205 & 0.1472 \\
\hline 5 & 0.6500 & 1.7627 & 0.5727 & 0.4123 & 0.7907 & 0.2078 \\
\hline 6 & 0.5714 & 1.8642 & 0.5313 & 0.4680 & 0.7772 & 0.1768 \\
\hline 7 & 0.6000 & 1.9639 & 0.6046 & 0.4043 & 0.8360 & 0.2279 \\
\hline 8 & 0.5833 & 1.8532 & 0.6180 & 0.4138 & 0.8617 & 0.2332 \\
\hline 9 & 0.5833 & 1.9477 & 0.6163 & 0.4066 & 0.8581 & 0.2347 \\
\hline 10 & 0.5714 & 1.8726 & 0.6063 & 0.4800 & 0.8659 & 0.2215 \\
\hline
\end{tabular}

We have explored content-based clustering of web documents using $k$-means and global $k$-means. In the next chapter we turn towards performing supervised classification of the same document sets using the $k$-Nearest Neighbor algorithm. 


\section{Chapter Nine}

\section{A Graph-Theoretical Extension of the k-Nearest Neighbors Classification Algorithm}

\section{$9.1 \quad$ Introduction}

Automated classification techniques, where new, previously unseen data items are categorized to a predefined class of similar items, has long been an active research area in pattern recognition, machine learning, and data mining. Manual classification can be costly due to the large number of instances to be checked, their complexity, or an insufficient amount of expert domain knowledge required to perform the classification. The benefit of automated systems in application domains where this occurs is obvious. Classification of natural language documents, such as web documents, is one such domain. Because the number of documents being produced now is more than ever before, especially when we consider the Internet with its massive amount of heterogeneous documents, manual classification and categorization can be extremely difficult.

Classification is different than the clustering procedures we previously examined for two major reasons. First, classification is a supervised learning task, meaning the classifier is first trained by exposing it to a set of labeled example data. Only after sufficient training is the classifier ready to be used for classification. Second, classification assigns a label to each data item (web document). In contrast clustering creates a series of groupings of the data. Thus the performance of clustering and classification algorithms is measured in different ways.

In this chapter we introduce a graph-based extension of the popular $k$-Nearest Neighbors $(k-\mathrm{NN})$ classification algorithm. The leave-one-out approach will be used to compare classification accuracy over our three document collections. We will select several values for the number of nearest neighbors, $k$, and will also look at the performance as a function of the size of the graphs representing each document. We will also compare the performance of different graph theoretical distance measures and the various methods of representing the web documents using graphs described in Chapter 4, much as we did for $k$-means.

The remainder of this chapter is organized as follows. We describe the graphbased extension of the $k-\mathrm{NN}$ algorithm in Section 9.2. In Section 9.3 we present the results of our experiments. Finally some concluding remarks are given in Section 9.4.

\section{2 $k$-Nearest Neighbors with Graphs}

In this section we describe the $k$-Nearest Neighbors $(k-\mathrm{NN})$ classification algorithm and how we can easily extend it to work with graph-based data. The basic $k$ NN algorithm is given as follows (see Fig. 9.1) [91]. First, we have a data set of training 
examples (sometimes also called training instances). In the traditional $k$-NN approach these will usually be numerical vectors in some real-valued Euclidean feature space. Each of these training instances is associated with a label which indicates to what class the instance belongs. Given a new, previously unseen instance, called a query or input instance, we attempt to estimate which class it belongs to. Under the $k$-NN method this is accomplished by looking at the $k$ training instances closest (i.e. with least distance) to the input instance. Here $k$ is a user provided parameter and distance is usually defined to be the Euclidean distance. Once we have found the $k$ nearest training instances using some distance measure, such as one of those defined in Eqs. 6.4-6.6, we estimate the class by the majority among the $k$ instances. This class is then assigned as the predicted class for the input instance. If there are ties due to more than one class having equal numbers of representatives amongst the nearest neighbors we can either choose one class randomly or we can break the tie with some other method, such as selecting the tied class which has the minimum distance neighbor. For the experiments in this chapter we will use the latter method, which in our experiments has shown a slight improvement over random tie breaking in nearly all cases. $k$-NN is classified as a lazy, instance-based learning algorithm. Lazy meaning learning is not actually performed until a new query instance is encountered, and instance-based meaning the knowledge utilized by the method is stored as the instances themselves rather than as rules, tables, or some other format.

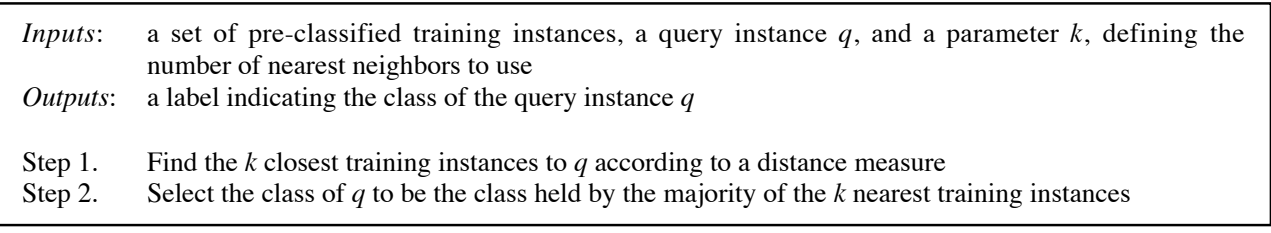

Figure 9.1. The Basic $k$-Nearest Neighbors Algorithm

The extension to using graphs as data for the $k$-NN algorithm is straightforward: we simply represent the data as graphs (Chapter 4) and use a graph-theoretical distance measure (Eqs. 3.3.-3.7) in lieu of traditional vector-based distance measures such as Euclidean distance. Otherwise the algorithm remains unchanged from its usual form. As discussed in the next section, we have implemented the $k$-NN algorithm for both the traditional case of representing data as numeric vectors and our approach where we represent data as graphs.

\subsection{Experimental Results}

For our graph-based experiments we used several values of maximum graph size to examine the effect of graph size on performance. Classification accuracy was measured by the leave-one-out method. In this method we iterate over all $n$ documents, using $n-1$ documents as training instances and then classifying the remaining instance. Accuracy is reported as the number of documents classified correctly divided by $n$. As we mentioned in Section 6.3, the F-series contains four classes: manufacturing, labor, business \& finance, and electronic communication \& networking; the J-series contains 
ten classes: affirmative action, business capital, information systems, electronic commerce, intellectual property, employee rights, materials processing, personnel management, manufacturing systems, and industrial partnership; and the K-series has 20 classes: business, health, politics, sports, technology, entertainment, art, cable, culture, film, industry, media, multimedia, music, online, people, review, stage, television, and variety.

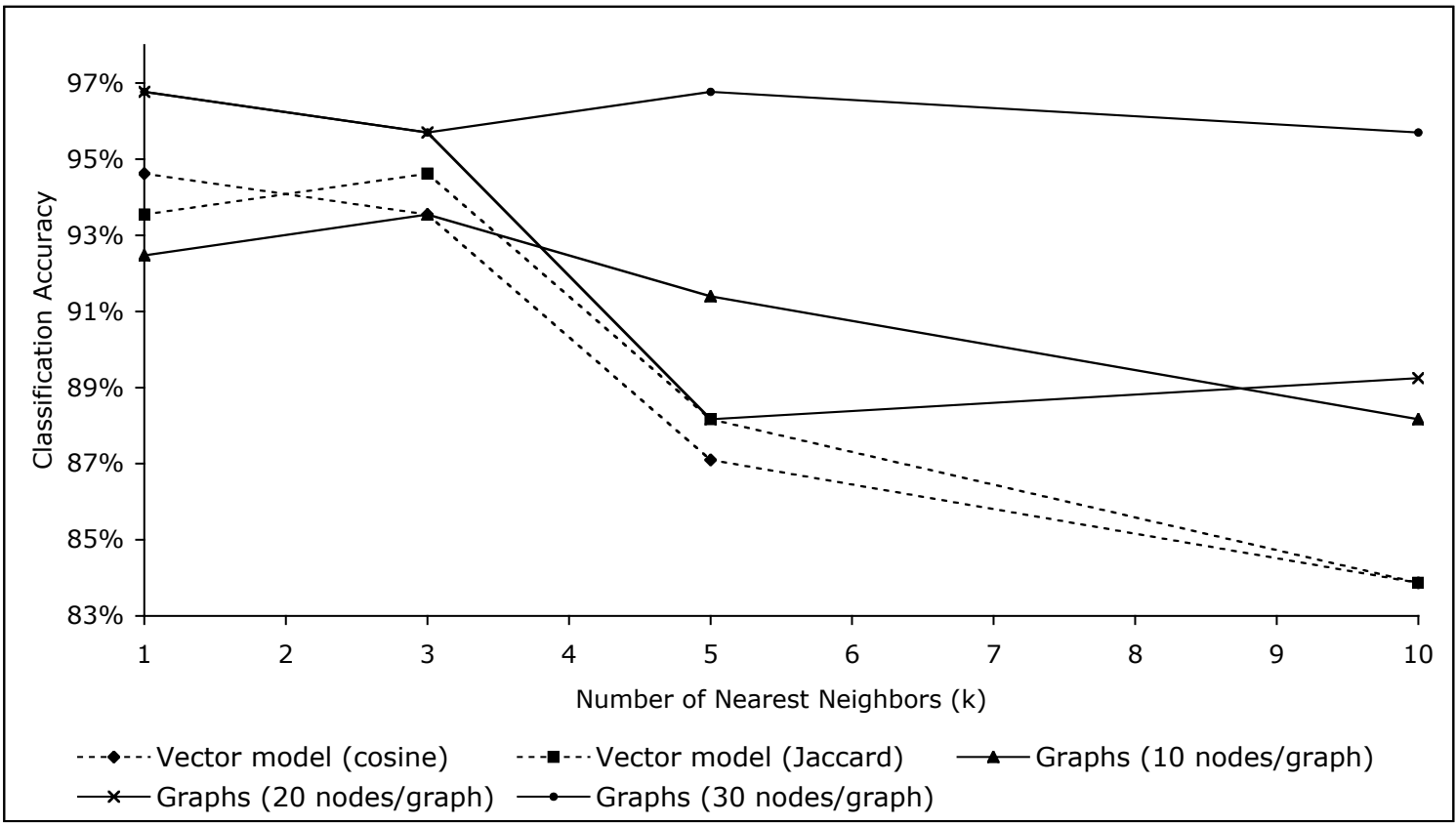

Figure 9.2. Classification Accuracy for the F-Series Data Set

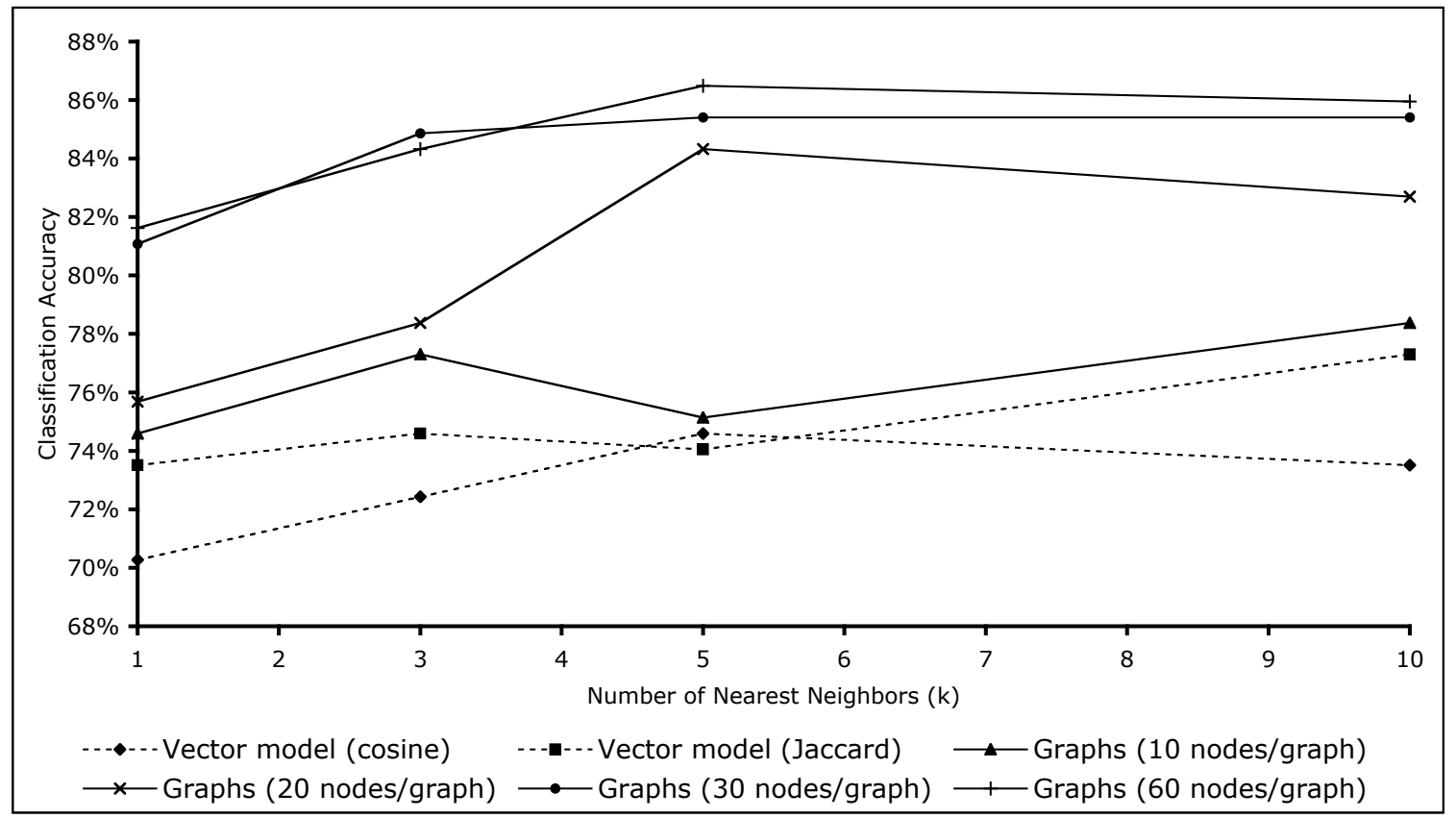

Figure 9.3. Classification Accuracy for the J-Series Data Set 
The results for the F-series, the J-series, and the K-series are given in Figs. 9.2, 9.3, and 9.4, respectively. The graphs show the classification accuracy as a function of $k$, the number of nearest neighbors to use. We used values of $1,3,5$, and 10 for $k$. The dotted lines in the figures indicate the performance of the vector model approach when using the cosine distance measure (Eq. 6.5) or extended Jaccard distance measure (Eq. 6.6), which we take here to be benchmarks against which our novel graph-based method is compared. The graph-based methods in these figures use the MCS distance measure (Eq. 3.3) and the "standard" graph representation (see Chapter 4).

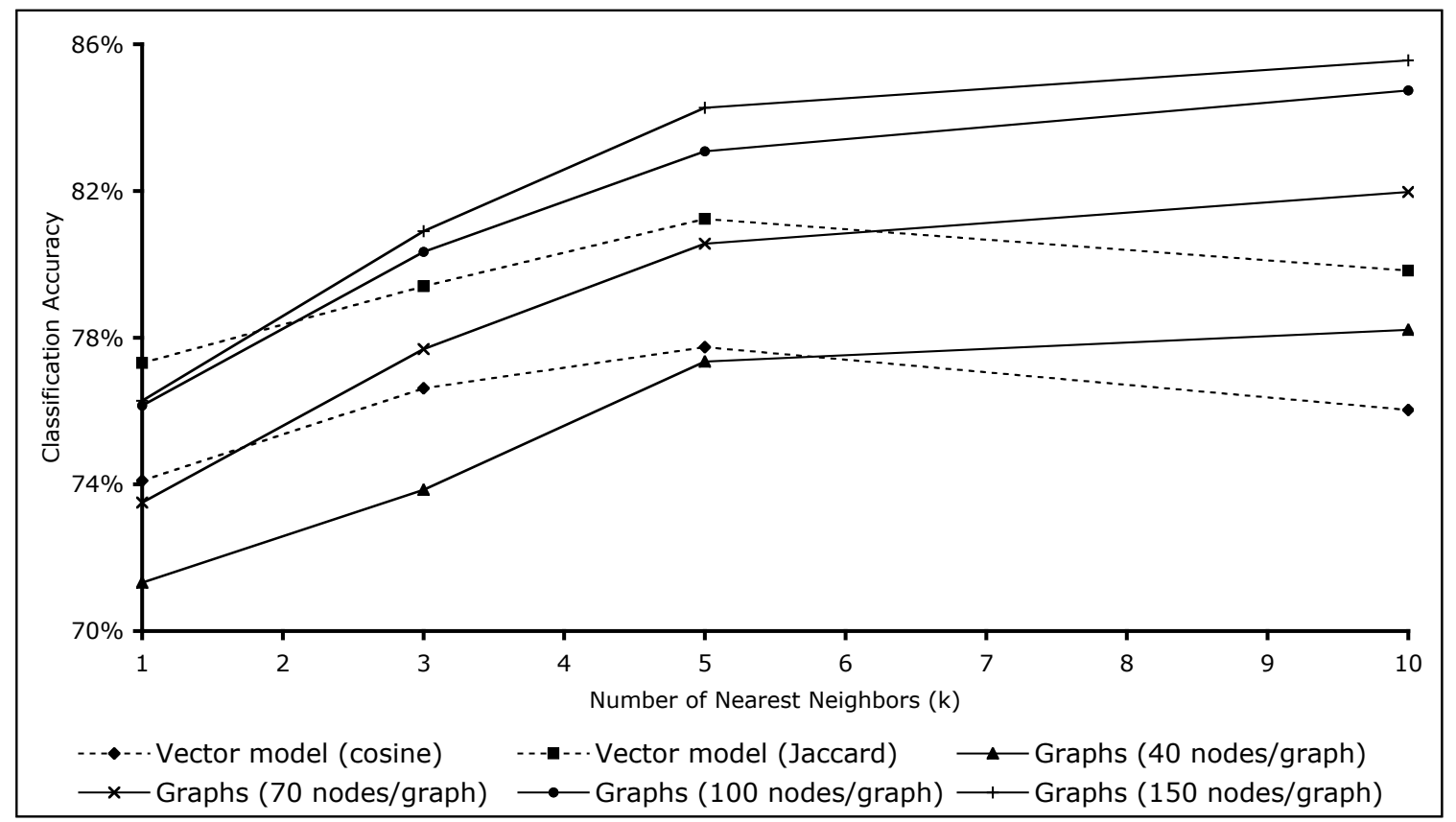

Figure 9.4. Classification Accuracy for the K-Series Data Set

We see that in the $\mathrm{F}$ and $\mathrm{J}$-series graphs of as few as 10 nodes usually outperformed the vector-based methods, regardless of the number of nearest neighbors. We also see that the performance continued to improve as we created larger graphs, with graphs using up to 20 or 30 nodes outperforming the usual vector methods in nearly all cases. The K-series, however, needed larger graph sizes to attain an improvement in accuracy over the vector model. We attribute this to the greater number of classes and documents used in this data set (twice as many classes as the J-series, and more than ten times the number of documents). In order to properly differentiate between classes a larger number of terms is necessary. This is also reflected in the vector model, which uses a much higher dimensionality for the K-series than the other two data sets $(1,458$ terms for $\mathrm{K}$ versus 332 for $\mathrm{F}$ and 474 for $\mathrm{J}$ ). For the K-series we used graph sizes of 40, 70, 100 and 150 nodes per graph maximum. At 40 nodes per graph our method performed similarly to but slightly better than the vector approach using cosine distance (comparing the best case performance for each method). With 70 nodes per graph the best performance using graphs was similar to the best performance using Jaccard. With 100 
nodes per graph the graph method outperformed both of the vector methods for values of $k \geq 3$ (three out of four cases). In all three document collections, the graph representations that outperformed the vector model were based on a significantly smaller (by an order of magnitude) number of terms. We have also observed that the number of nearest neighbors $(k)$ has no consistent effect on the classification performance of any model (whether vector or graph based) for the F and J-series. For the K-series, however, we saw a trend of increasing performance with larger $k$ values for the graph-based method. This is likely due to the larger number of documents contained in this data set. The vectorbased methods showed a decline in performance as $k$ became larger than 5 .

Table 9.1. Average Times to Classify One K-Series Document for Each Method

\begin{tabular}{|c|c|}
\hline Method & Average time to classify one document \\
\hline Vector (cosine) & 7.8 seconds \\
\hline Vector (Jaccard) & 7.79 seconds \\
\hline Graphs, 40 nodes/graph & 8.71 seconds \\
\hline Graphs, 70 nodes/graph & 16.31 seconds \\
\hline Graphs, 100 nodes/graph & 24.62 seconds \\
\hline
\end{tabular}

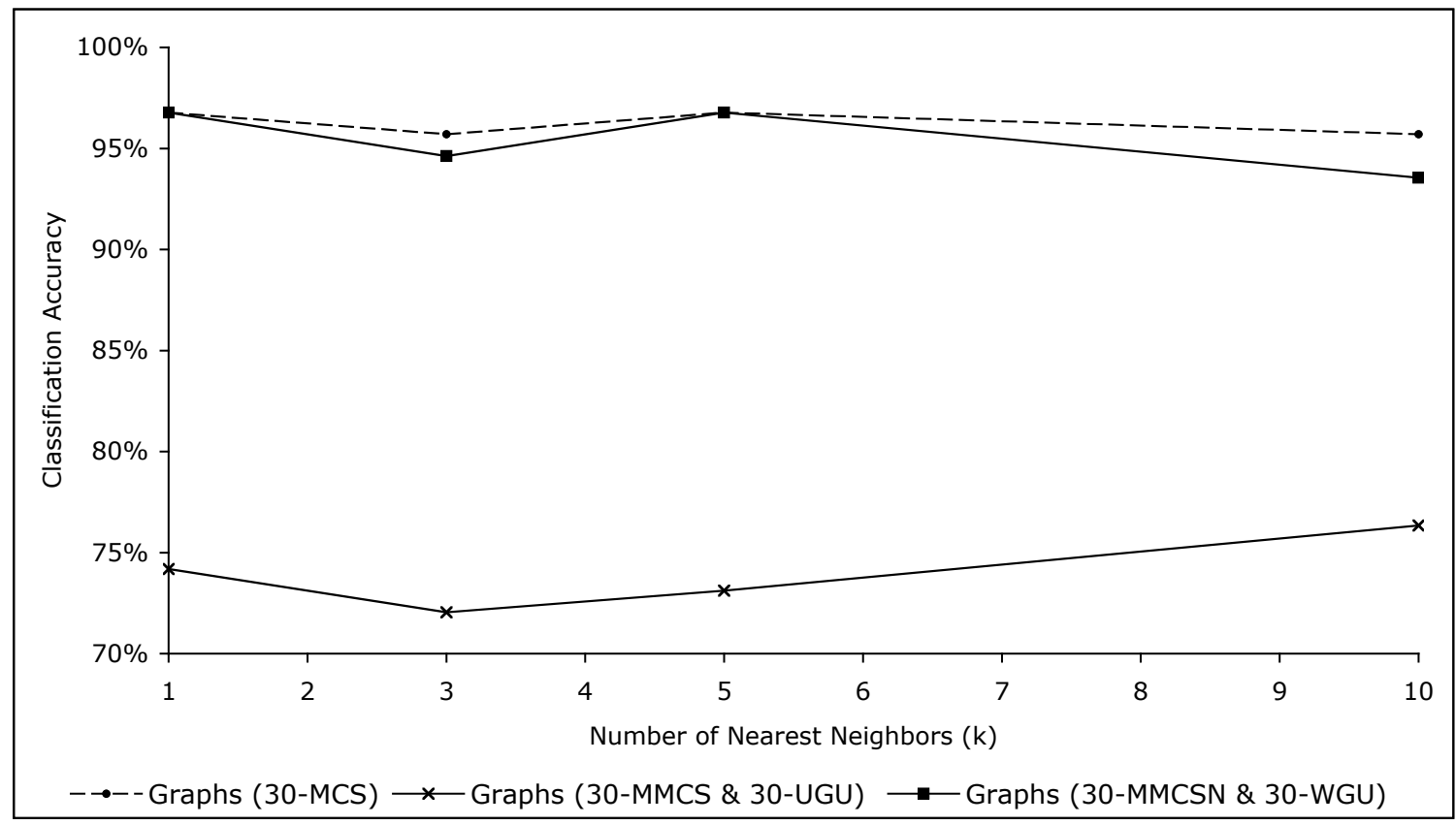

Figure 9.5. Distance Measure Comparison for the F-Series

We also measured the execution times needed to perform the classification for the $\mathrm{K}$-series data set, which was the most time-consuming, for both the vector model approach and our graph-based approach. The methods were timed for $k=10$, and the experiments were carried out on the same system under the same operating conditions (a 
cluster of seven $2.6 \mathrm{GHz}$ Pentium 4 systems each with 1 gigabyte of memory). The average times to classify a document for each method, measured over 100 experiments, are shown in Table 9.1. It is interesting to note the relationship between run time and performance for our method: as graph sizes become larger the performance increases but so does the time needed to perform the classification. The classification times for our method are also similar to those of the vector model for smaller graph sizes.

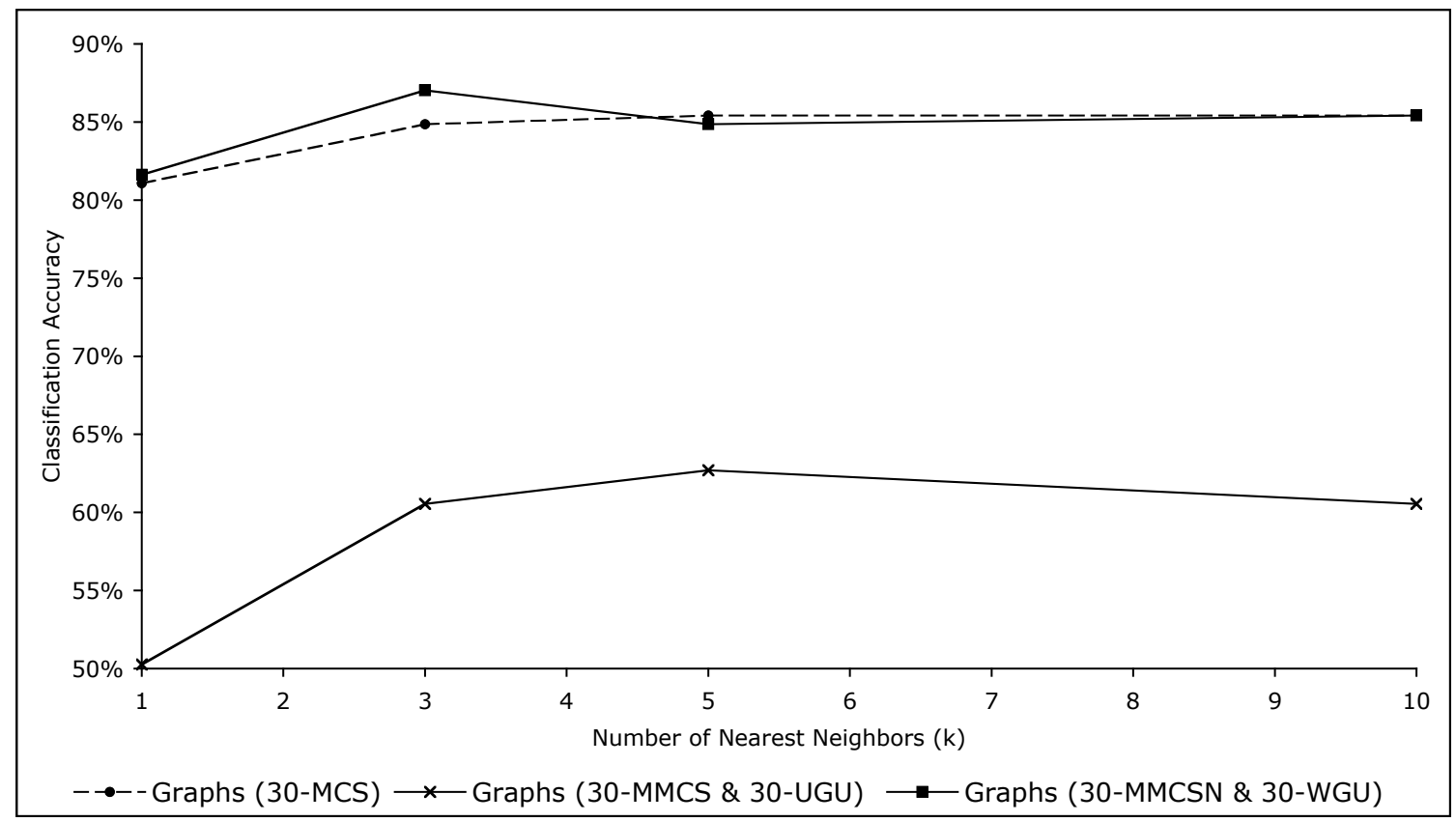

Figure 9.6. Distance Measure Comparison for the J-Series

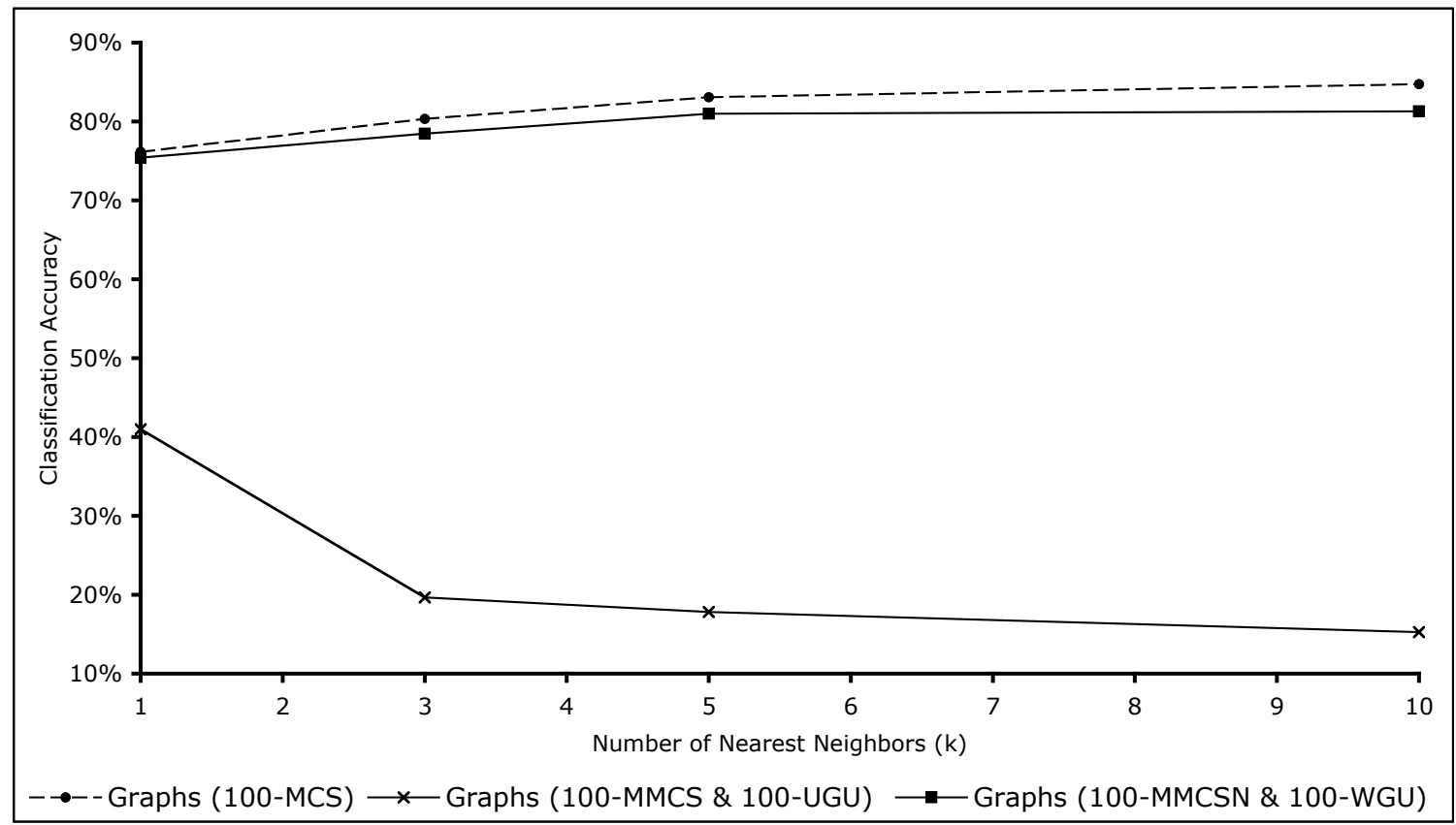

Figure 9.7. Distance Measure Comparison for the K-Series 
In Figs. 9.5-9.7 we show the comparison of different graph distance measures when classifying the F, J, and K-series, respectively. The MCS distance, which was used in Figs. 9.2-9.4, is shown as a dotted line. The results of each distance measure are based on the same set of best performing graphs (30 nodes per graph maximum for $\mathrm{F}$ and $\mathrm{J} ; 100$ for K) for each data set. We see that the graph-based methods that use normalized distance measures performed well, while distance measures that were not normalized to the interval $[0,1]$ performed poorly. This same behavior was also noted in Chapter 7 for clustering performance.

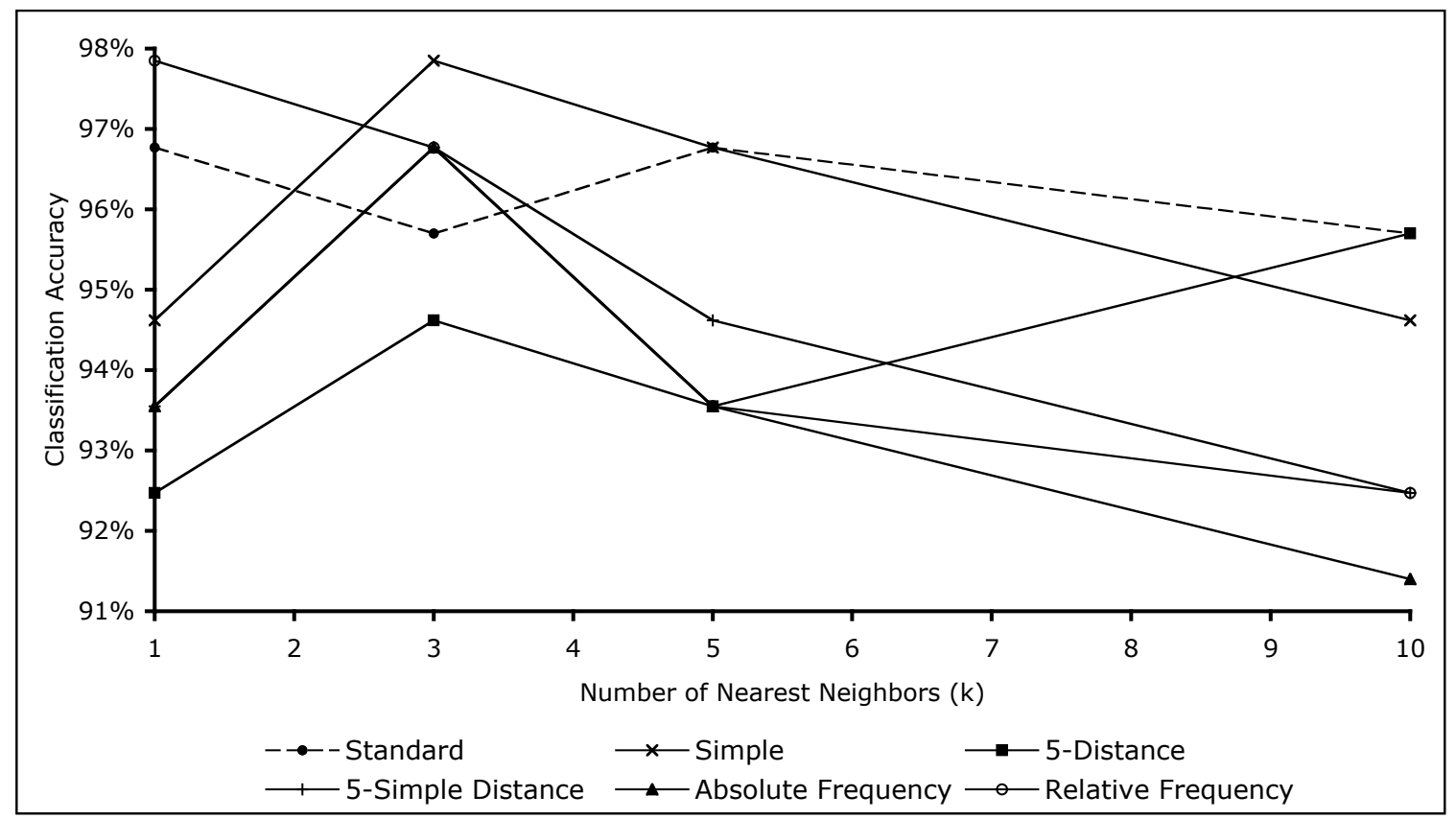

Figure 9.8. Graph Representation Comparison for the F-Series

In Figs. 9.8-9.10 we give the results of the comparison of the different graph representations we proposed for the F, J, and K-series, respectively. The dotted line indicates the standard representation, which was what was used in Figs. 9.2-9.4. We use the same graph sizes as we used previously in the distance measure comparison (Figs. 9.5-9.7). Here, as before, we use $\mathrm{n}=5$ for our distance related representations, i.e. 5-distance and 5-simple distance. There are some interesting trends that are apparent in these graphs. We see that while the standard representation performed well in many cases, in the $\mathrm{F}$ and $\mathrm{J}$ data sets the simple representation produced the best performance of all the methods (at $\mathrm{k}=3$ for the F-series and $\mathrm{k}=5$ for the $\mathrm{J}$-series) while for the $\mathrm{K}$-series the absolute frequency representation was clearly the best for all values of k. For the F and J-series this could indicate that the simple representation can be just as good or better than the standard representation, but is more sensitive to the value of $k$. Note that in both cases $(\mathrm{F}$ and $\mathrm{J})$ the standard representation outperformed the simple representation for $\mathrm{k}=$ 1 , which suggests that the standard method actually creates a representation that is better when we compare two data items in the pair-wise sense; i.e. the standard method creates 
graphs that when compared to each other provide a more accurate distance value, and it is only when we introduce larger neighborhoods of graphs that the simple representation can be more effective than the standard. The relative frequency representation performed well for the F-series at $\mathrm{k}=1$, but its performance declined with larger $\mathrm{k}$ values. For the Jseries, the opposite trend was present for relative frequency: larger values of $\mathrm{k}$ lead to increasingly better performance.

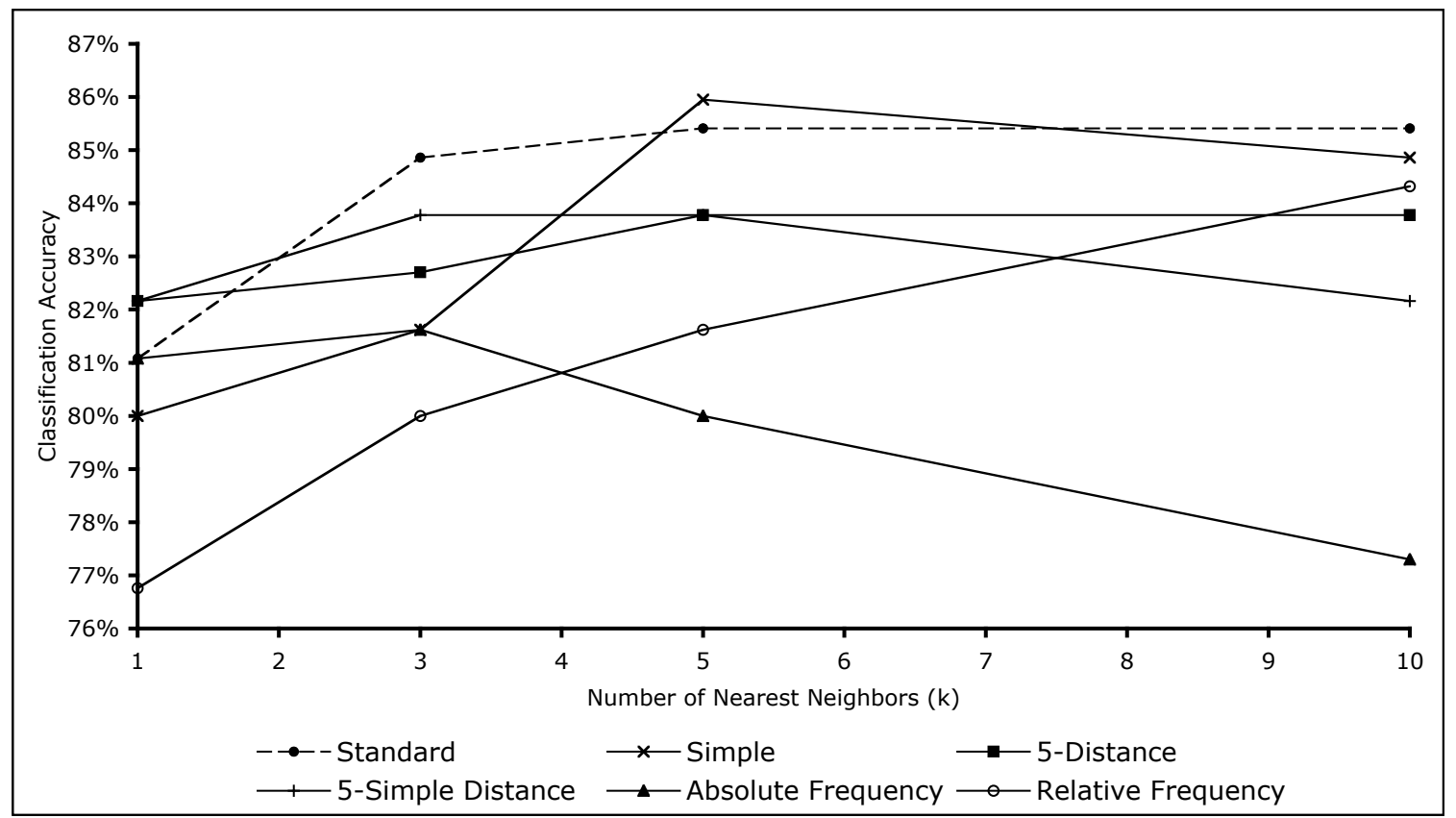

Figure 9.9. Graph Representation Comparison for the J-Series

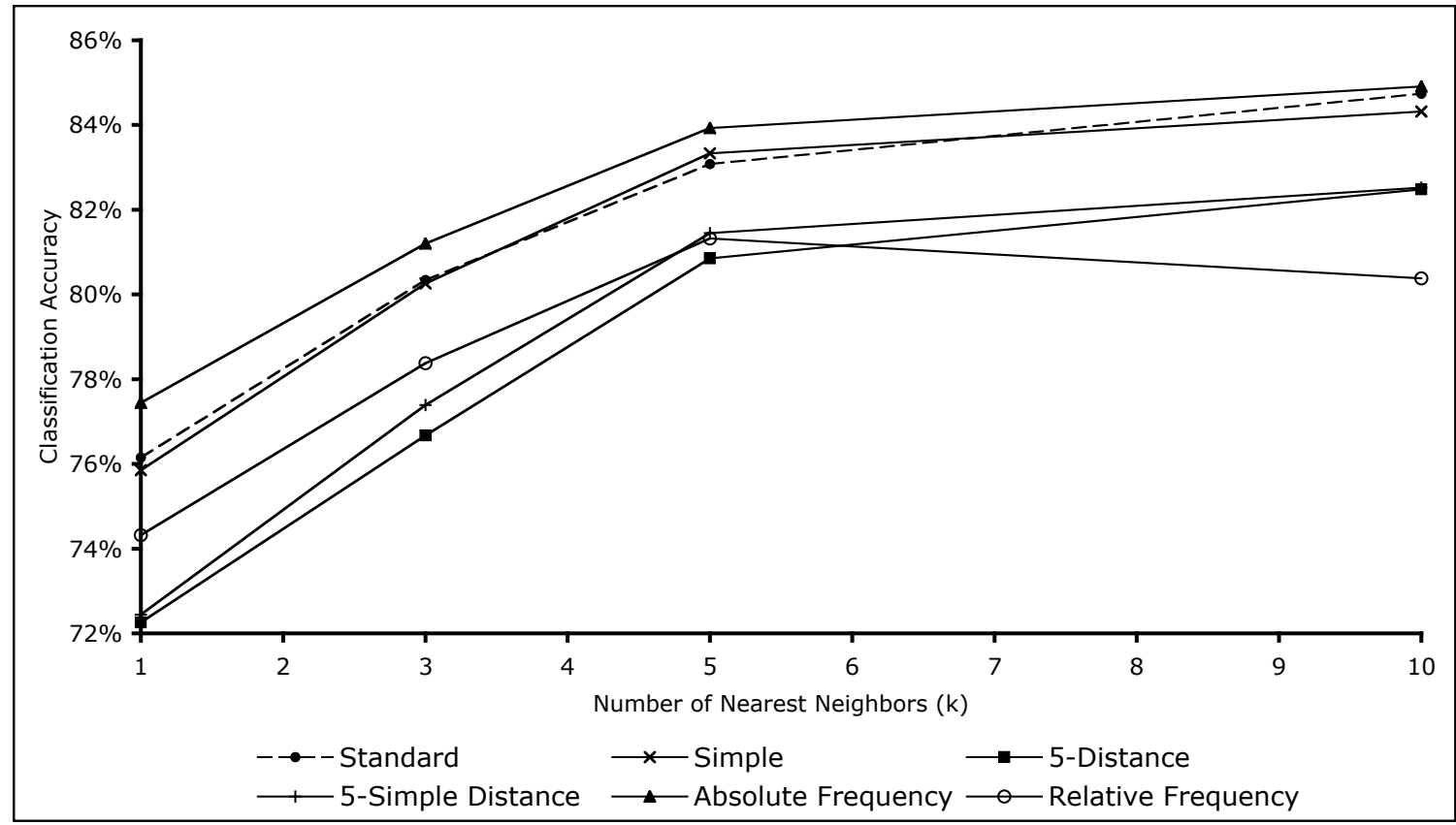

Figure 9.10. Graph Representation Comparison for the K-Series 
For the K-series, the superior performance of the absolute frequency representation is possibly due to the fact that this is a highly homogeneous data set. Compared to the F and J-series data sets, whose documents are authored by different people and contain variations in style, layout, and structure, the K-series documents have similar layout, structure and size due to being formatted and hosted by a single source (Yahoo). Every document contains an identical subset of terms (e.g. "news", "sports", etc.) that comes from elements such as menus and navigation links. Using the absolute frequency representation may help differentiate document content under these conditions. For example, the term "news" may appear on every document because it appears as a navigational link, but if it appears twenty times instead of three this may be significant for classification purposes. Alternatively, a term such as "news" appearing on a document may have a low frequency due to it coming from a navigational link. Thus the importance of such terms when calculating document similarity is less than other terms with higher frequency (those that are content-related). This information is captured in the frequencyrelated representations but not the other graph representations. The relative frequency representation did not perform as well as absolute frequency for the K-series, indicating that normalization of frequency values was not necessary for this homogeneous data set.

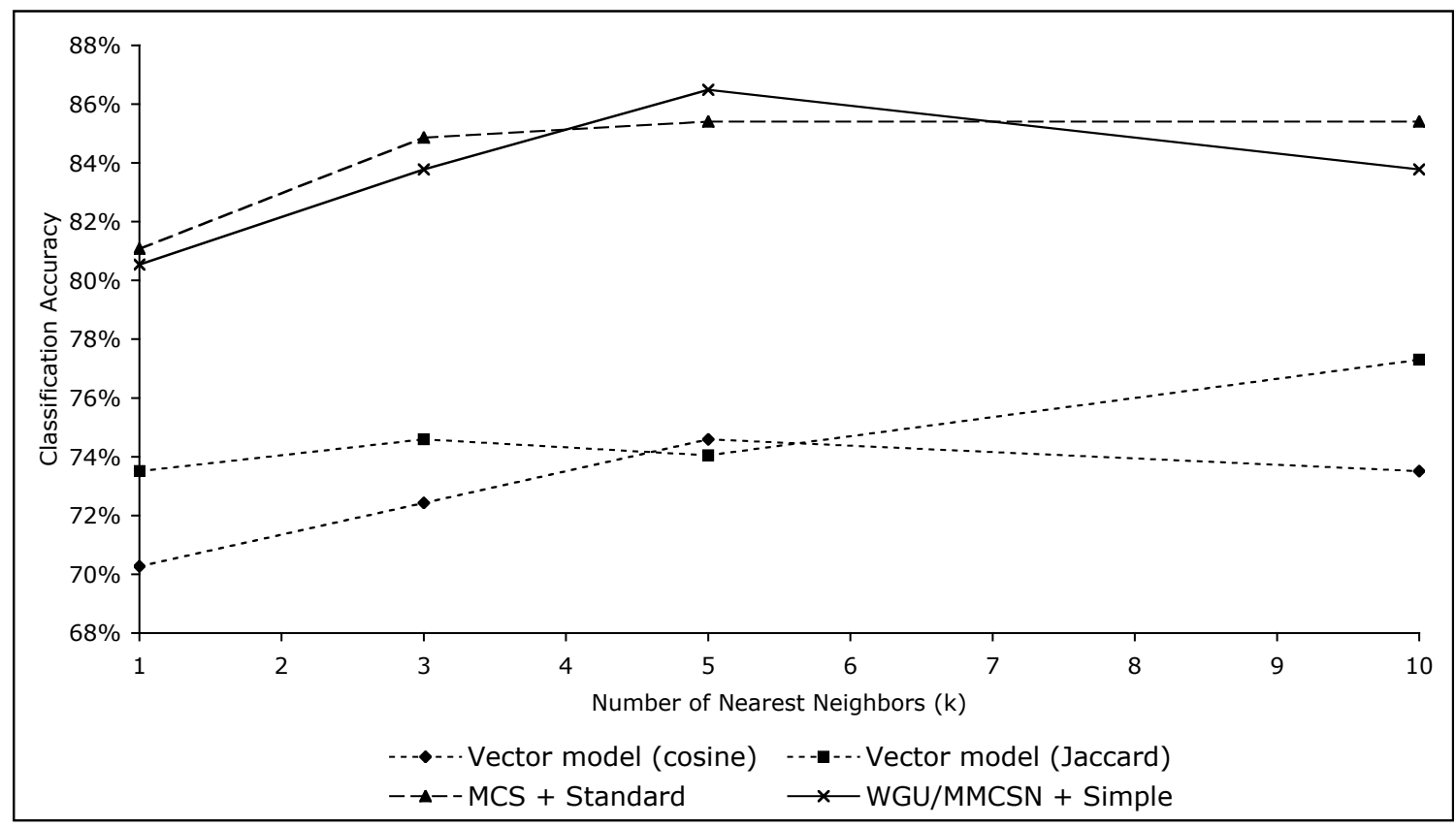

\section{Figure 9.11. Combining WGU/MMCSN Distance Measure and Simple Representation for the J-Series}

In the experimental results above we saw that while the MCS distance and standard representation were consistently among the top performing methods in many cases, for the J-series the WGU/MMCSN distance measure (Fig. 9.6, 87.03\% for $k=3$ ) and simple graph representation (Fig. 9.9, 85.95\% for $k=5$ ) were the best performing overall (the best performance overall being indicated by the data point that appears highest in the chart). In order to determine if we could obtain an even better performance 
for the J-series, we considered the case where we combine the best overall performing distance measure (WGU/MMCSN) and the best overall performing graph representation (simple). Note that for both the F-series and the K-series the best overall distance measure was MCS, and the results of combining the MCS distance measure with the various representations is already given in Figs. 9.8 and 9.10. The outcome of combining the WGU/MMCSN distance measure with the simple representation for the J-series is given in Fig. 9.11. We see that we obtained a slightly better result than the combination of MCS and standard for $k=5(86.49 \%)$, which was also the best overall performance for the $\mathrm{J}$-series for $k=5$. However, neither of the two combinations has a consistent advantage over the other one.

\section{$9.4 \quad$ Remarks}

In this chapter we have examined the problem of classifying web documents when the data items are represented by graphs instead of simpler feature vectors. To perform the classification we developed a graph-based version of the $k$-Nearest Neighbors classification algorithm, substituting a suitable graph-theoretical distance measure in the place of the usual vector-related distance. Experimental results showed that the graph-based method can outperform the traditional vector methods in both terms of accuracy and execution time. We saw that graph distance measures that were not normalized performed poorly, while those that were normalized to the interval $[0,1]$ yielded good results. For two heterogeneous collections of web documents we saw the "standard" graph representation (all text including keyword and title information with edge labels referring to document sections) perform well overall, though the "simple" method (visible text only, section information ignored) achieved the best performance for some values of $k$ in both collections. In another, highly homogenous collection, the absolute frequency representation was the best performing. The value of the $k$ parameter had no consistent effect on the performance for most representations and distance measures.

A surprising result is that not only can the graph-based method be more accurate than the vector model, it can also be similar in terms of classification time even though graph similarity is a more expensive computation than vector distance $\left(O\left(n^{2}\right)\right.$ for graphs compared to $O(n)$ for vectors). This is due in part to the increased representational power of graphs over vectors: we only need a relatively few terms (nodes) per graph in comparison to the number of dimensions used in the vector case. Further, since the vector model requires that each vector representing a document include exactly the same set of terms, this leads to overhead in execution time for the distance comparison of all documents whether or not each term is actually useful for every document (i.e. incorporating a new term always increases the dimensionality of all vectors by one). In contrast, our graph-based method allows us to define specific terms for only a select group of graphs by simply adding relevant nodes and edges to those graphs while leaving the others unchanged. Consequently, $n$ graphs each with $m$ nodes can contain information relating to up to $n \cdot m$ distinct terms, while $n$ equal-size vectors with $m$ dimensions in a term-document matrix refers to $m$ terms only. Given this observation it may be possible to attain even better time savings by selecting the group of terms modeled in each graph 
more carefully. We could, for example, allow hard-to-classify documents to be represented by larger graphs while using a minimal representation for others.

To conclude our remarks concerning graph representations under the $k$-NN algorithm, we mention that in Appendix A some examples of actual web documents used in our experiments are provided for inspection. The HTML source of three documents randomly taken from the J-series collection is given in Figs. A.1-A.3. These documents are, respectively:

(1) document \#68, a press release originally from http://www.executone.com/ pressrel/vxc.htm which is classified as an information systems document

(2) document \#104, an employee rights document originally from http://riles. alameda-coe.k12.ca.us/online/Dubl/Cert/2/EmplRi.html

(3) document \#183, a document describing a conference from http://www. mf.polyu.edu.hk/seccabw.htm classified as a manufacturing systems document

The documents as they would appear when rendered in a web browser are shown in Figs. A.4-A.6. The corresponding graphs created for each of these documents, constructed using the standard representation and a 10 node per graph limit, are given in Appendix B (Figs. B.1-B.3). Note that some graphs have fewer than ten nodes due to stemming and subsequent conflation. The HTML source of the nearest neighbor document of each example document in Appendix A, determined through the minimum MCS distance, is given in Appendix C (Figs. C.1-C.3); the rendered versions are given in Figs. C.4-C.6. The corresponding standard graph representations of the nearest neighbor documents in Appendix C are given in Appendix D (Figs. D.1-D.3). 


\section{Chapter Ten}

\section{Conclusions and Future Work}

In this dissertation we have introduced several new techniques for performing web content mining tasks when utilizing more descriptive graphs in lieu of the usual case of vector representations. Our first contribution is presenting a number of ways by which web document content can be modeled as graphs. These graph representations retain information that is usually lost when using a vector model, such as term order and document section information. We demonstrated how with careful selection of a graph representation, namely a representation with unique node labels, we can perform the graph similarity task in $O\left(n^{2}\right)$ time ( $n$ being the number of nodes). In general, graph similarity using maximum common subgraph is an NP-Complete problem, so this is an important result that allows us to forgo sub-optimal approximation approaches and find the exact solution in polynomial time.

Next we introduced the Graph Hierarchy Construction Algorithm (GHCA): a novel, iterative hierarchical clustering algorithm which labels clusters according to topic. GHCA was implemented as part of a system that automatically organized web documents returned by conventional web search systems in order to allow for easier browsing of the results and examination of related topics. Some useful benefits of this novel clustering algorithm are: 1. labeling of clusters by topic in order to convey each cluster's purpose to the user, 2. the hierarchical ordering organizes clusters from the most general to the most specific in order to allow users to "drill down" to their desired level of specificity, 3 . we allow for clusters to have multiple parents and for assignment of pages to clusters to be either exclusive or non-exclusive, 4 . we provide several parameters that the user to tailor the behavior of the algorithm, such as limiting the maximum number of clusters created, and 5. the graph representations used provide term ordering and phrase information which permits a better display of cluster (topic) labels. Due to some special circumstances that make comparison with ground truth difficult, such as the fact that clusters are labeled by topic, the performance of our system was evaluated by comparing it with two similar search clustering systems. The results show our search clustering system compared favorably to the similar systems.

Another contribution of this work is far more wide reaching: we extended classical, well-known machine learning techniques, such as the $k$-means clustering algorithm and $k$-Nearest Neighbors classification algorithm, to allow them to work directly with graphs as data items, instead of more limited vectors. This is a major contribution because: 1. it allows for complex, structured data, such as web documents, to be represented by a more robust model that has the potential to retain information that is usually discarded when using a vector representation and 2. we can use many existing, proven machine learning algorithms with graphs without having to create new, 
specialized ones. This opens up the possibility of using a variety of different techniques with graph-based data, where previously sets of atomic (often purely numeric) data were required due to theoretical limitations. Because the extended graph-theoretical versions of these well-known algorithms do not limit the form of the graphs, they are applicable to any graph-based representation of data. Thus we can change graph representations or even application domains without reformulating the underlying algorithms.

In this dissertation we modeled web documents as graphs and performed experiments comparing the performance of clustering and classification when using the traditional vector representation and our novel graph-based representations. We introduced several different graph representations and five graph-theoretical distance measures. Experiments were performed on three web document data sets and performance was measured using clustering performance measures as compared to ground truth, cluster validity indices (such as the Dunn index), or accuracy measured by leave-one-out (for classification procedures). Experimental results consistently show an improvement in performance over the comparable vector-based methods when using graph-based approaches. In addition, some of our experiments also showed an improvement in execution time over the vector model.

A number of exciting avenues of future work related to graph-theoretic machine learning exist. First, other machine learning algorithms can be adapted to work with graph-based data. For example, a graph-theoretic version of the popular fuzzy $c$-means algorithm, which is a partitional clustering algorithm which assigns fuzzy membership values in the interval $[0,1]$ to each item in a data set, could be created. These values indicate the degree of membership of the data item in each cluster; higher values indicate stronger membership. The typical fuzzy $c$-means algorithm works as follows [61]. First, a fuzzy partition matrix $A$ is generated randomly. Entry $a_{i j}$ in matrix $A$ indicates the membership of data item $i$ in cluster $j$. From this fuzzy matrix cluster centers $v_{j}$ are calculated by the following equation:

$$
v_{j}=\frac{\sum_{k=1}^{n}\left[A_{j}\left(x_{k}\right)\right]^{m} x_{k}}{\sum_{k=1}^{n}\left[A_{j}\left(x_{k}\right)\right]^{m}}
$$

where $x_{k}$ is the vector representing the $k$ th data item (out of $n$ total data items) and $m$ is a user provided parameter that controls the behavior of the algorithm. Next the fuzzy matrix $A$ is updated from the new cluster centers by:

$$
a_{i j}=\left[\sum_{k=1}^{c}\left(\frac{d\left(x_{j}, v_{i}\right)}{d\left(x_{j}, v_{k}\right)}\right)^{\frac{1}{m-1}}\right]^{-1}
$$

where $c$ is the number of clusters and $d(x, v)$ is the distance between data item $x$ and cluster center $v$ (usually defined as the Euclidean distance). The cluster centers and fuzzy partition matrix are alternately recomputed using the same procedure, until the maximum change in successive fuzzy matrices is less than or equal to some user provided value $\varepsilon$. 
At that point the algorithm is terminated and the matrix $A$ contains the induced fuzzy partition that represents the clustering of the data set. The main challenge with adapting fuzzy $c$-means for graphs lies in creating a method of computing the cluster centers. Under fuzzy $c$-means the cluster centers are computed with a weighted averaging that takes into account the membership values of each data item. Thus the graph median cannot be directly used as was done with $k$-means.

Second, new graph representations which may further improve performance can be envisioned. We previously described several different methods of representing web documents using graphs. It is possible to create other, more elaborate representations that include even more information, such as information about HTML tags or document elements such as sentences, paragraphs, sections, tables, images, lists and so forth. Future experiments may compare the performance of such representations to the results presented here.

Third, as we saw earlier, multidimensional scaling combined with graphs promises to be an extremely interesting area of research. Scaling can be applied to graphbased data when using different graph representations and distance measures in order to visualize the impact each approach has. Other types of complex, structured data, such as software code, can also be visualized with this method. In addition to visualization, the Euclidean vector representation of the original data opens up the possibility of using a wide array of additional techniques on graph-based data, such as neural networks. Future experiments could compare, for example, graph-based $k$-means clustering performance to the performance of vector-based $k$-means when using the scaled graph-based data. The optimal number of dimensions to use during scaling is also an issue that needs to be addressed experimentally, as there is a trade-off between the number of dimensions and the amount of information lost during the scaling.

Finally, the methods developed here are applicable to many domains other than web document content. File directories, organizational charts, images, and networks are just some examples of domains that are naturally modeled by graphs. Now that we have introduced methods that allow standard machine learning algorithms to deal with graphbased representations, these types of data can be handled directly, without having to discard the structural information in favor of a simpler vector model or create new theoretical models of the particular domain. 


\section{References}

1. M. Agosti and A. Smeaton (Eds.), Information Retrieval and Hypertext, Kluwer Academic Publishers, Norwell, MA, 1996.

2. R. Agrawal, R. J. Bayardo Jr. and R. Srikant, "Athena: Mining-based Interactive Management of Text Databases", Proceedings of the $7^{\text {th }}$ Conference on Extending Database Technology, 2000.

3. H. Ahonen, O. Heinonen, M. Klemettinen, and A. I. Verkamo, "Applying Data Mining Techniques in Text Analysis", Technical Report C-1997-23, University of Helsinki, Department of Computer Science, March 1997.

4. C. Apte, F. Damerau, and S. M. Weiss, "Automated Learning of Decision Rules for Text Categorization", ACM Transactions on Information Systems, Vol. 12, 1994, pp. 233-251.

5. J. G. Augustson and J. Minker, "An Analysis of Some Graph Theoretical Cluster Techniques", Journal of the Association of Computing Machinery, Vol. 17, No. 4, October 1970, pp. 571-588.

6. R. Baeza-Yates and B. Ribeiro-Neto, Modern Information Retrieval, AddisonWesley, Wokingham, UK, 1999.

7. D. Beeferman and A. Berger, "Agglomerative Clustering of a Search Engine Query Log", Proceedings of the $6^{\text {th }}$ International Conference on Knowledge Discovery and Data Mining ACM SIGKDD, 2000.

8. D. Boley, M. Gini, R. Gross, E. H. Han, K. Hastings, G. Karypis, B. Mobasher, J. Moore, "Partitioning-based Clustering for Web Document Categorization", Decision Support Systems, Vol. 27, 1999, pp. 329-341.

9. H. Bunke, "On a relation between graph edit distance and maximum common subgraph", Pattern Recognition Letters, Vol. 18, 1997, pp. 689-694.

10. H. Bunke, "Error Correcting Graph Matching: On the Influence of the Underlying Cost Function", IEEE Transactions on Pattern Analysis and Machine Intelligence, Vol. 21, No. 9, September 1999, pp. 917-922.

11. H. Bunke, "Recent developments in graph matching", Proceedings of the $15^{\text {th }}$ International Conference on Pattern Recognition, Vol. 2, Barcelona, 2000, pp. 117-124.

12. H. Bunke, S. Günter, and X. Jiang, "Towards Bridging the Gap between Statistical and Structural Pattern Recognition: Two New Concepts in Graph Matching", in Advances in Pattern Recognition - ICAPR 2001, S. Singh, N. Murshed, and W. Kropatsch (Eds.), Springer Verlag, LNCS 2013, 2001, pp. 1-11. 
13. H. Bunke, X. Jiang, and A. Kandel, "On the minimum Common Supergraph of Two Graphs", Computing, Vol. 65, 2000, pp. 13-25.

14. H. Bunke and A. Kandel, "Mean and maximum common subgraph of two graphs”, Pattern Recognition Letters, Vol. 21, 2000, pp. 163-168.

15. H. Bunke and K. Shearer, "A graph distance metric based on the maximal common subgraph”, Pattern Recognition Letters, Vol. 19, 1998, pp. 255-259.

16. R. Burgin, "The Retrieval Effectiveness of Five Clustering Algorithms as a Function of Indexing Exhaustivity", Journal of the American Society for Information Science, Vol. 46, No. 8, 1995, pp. 562-572.

17. S. Chakrabarti, M. H. van den Berg, and B. E. Dom, "Distributed Hypertext Resource Discovery Through Examples", Proceedings of the $25^{\text {th }}$ International Conference on Very Large Databases, 1999, pp. 375-386.

18. C.-H. Chang and C.-C. Hsu, "Customizable Multi-engine Search Tool with Clustering”, Computer Networks and ISDN Systems, Vol. 29, 1997, pp. 1217-1224.

19. G. Chartrand, G. Kubicki, and M. Schultz, "Graph similarity and distance in graphs", Aequationes Mathematicae, Vol. 55, 1998, pp. 129-145.

20. J. Chen, A. Mikulcic, and D. H. Kraft, "An Integrated Approach to Information Retrieval with Fuzzy Clustering and Fuzzy Inferencing", in Knowledge Management in Fuzzy Databases, O. Pons, M. A. Vila and J. Kacprzyk (Eds.), Physica-Verlag, Heidelberg, 2000, pp. 247-260.

21. D. Conte, P. Foggia, C. Sansone, and M. Vento, "Thirty Years of Graph Matching in Pattern Recognition", to appear in International Journal of Pattern Recognition and Artificial Intelligence.

22. T. H. Cormen, C. E. Leiserson, and R. L. Rivest, Introduction to Algorithms, The MIT Press: Cambridge, Massachusetts, 1997.

23. T. M. Cover and J. A. Thomas, Elements of Information Theory, Wiley, 1991.

24. T. F. Cox and M. A. A. Cox, Multidimensional Scaling, Chapman and Hall, 1994.

25. F. Crimmins, A. Smeaton, T. Dkaki, and J. Mothe, "TétraFusion: Information Discovery on the Internet”, IEEE Intelligent Systems, Vol. 14, No. 4, 1999, pp. 55-62.

26. M. Crochemore and W. Rytter, Text Algorithms, Oxford University Press, New York, NY, 1994.

27. M. Crochemore and R. Vérin, "Direct Construction of Compact Directed Acyclic Word Graphs", in CPM97, A. Apostolico and J. Hein (Eds.), Springer-Verlag, LNCS 1264, 1997, pp. 116-129.

28. A. D. J. Cross, R. C. Wilson, and E. R. Hancock, "Inexact Graph Matching Using Genetic Search”, Pattern Recognition, Vol. 30, No. 6, 1997, pp. 953-970. 
29. D. B. Crouch, C. J. Crouch, and G. Andreas, "The Use of Cluster Hierarchies in Hypertext Information Retrieval", Proceedings of the ACM Hypertext '89 Conference, 1989, pp. 225-237.

30. D. Davies and D. Bouldin, "A cluster separation measure", IEEE Transactions on Pattern Recognition and Machine Intelligence, Vol. 1, No. 2, 1979, pp. 209-224.

31. P. De Boeck and S. Rosenberg, "Hierarchical Classes: Model and Data Analysis", Psychometrika, Vol. 53, No. 3, Sept. 1988, pp. 361-381.

32. S. Deerwester, S. Dumais, T. Furnas, T. Landaur and R. Harshman, "Indexing by Latent Semantic Analysis", Journal of the American Society for Information Science, Vol. 41, No. 6, pp. 391-407.

33. L. Denoyer and P. Gallinari, "A Belief Networks-Based Generative Model for Structured Documents. An Application to the XML Categorizaion”, Lecture Notes in Artificial Intelligence, Vol. 2734, 2003, pp. 328-342.

34. P. J. Dickinson, H. Bunke, A. Dadej, and M. Kraetzl, "Application of Median Graphs in Detection of Anomalous Change in Communication Networks", Proceedings of the World Multiconference on Systemics, Cybernetics and Informatics, Vol. 5, 2001, pp. 194-197.

35. J. Dunn, "Well separated clusters and optimal fuzzy partitions", Journal of Cybernetics, Vol. 4, pp. 95-104.

36. S. Dumais and H. Chen, "Hierarchical Classification of Web Content", Proceedings of SIGIR-00, $23^{\text {rd }}$ ACM International Conference on Research and Development in Information Retrieval, 2000, pp. 256-263.

37. M. Eirinaki and M. Vazirgiannis, "Web Mining for Web Personalization”, ACM Transactions on Internet Technology, Vol. 3, No. 1, 2003, pp. 1-27.

38. M. A. Eshera and K.-S. Fu, "A Graph Distance Measure for Image Analysis", IEEE Transactions on Systems, Man, and Cybernetics, Vol. SMC-14, No. 3, May/June 1984, pp. 398-408.

39. M.-L. Fernández and G. Valiente, "A graph distance metric combining maximum common subgraph and minimum common supergraph", Pattern Recognition Letters, Vol. 22, 2001, pp. 753-758.

40. A. M. Finch, R. C. Wilson, and E. R. Hancock, "An Energy Function and Continuous Edit Process for Graph Matching", Neural Computation, Vol. 10, 1998, pp. 1873-1894.

41. H. Frigui and R. Krishnapuram, "A robust algorithm for automatic extraction of an unknown number of clusters from noisy data", Pattern Recognition Letters, Vol. 17, 1996, pp. 1223-1232.

42. J. C. Gardin, SYNTOL, The Rutgers University Press, New Brunswick, NJ, 1965. 
43. S. Gold and A. Rangarajan, "A Graduated Assignment Algorithm for Graph Matching", IEEE Transactions on Pattern Analysis and Machine Intelligence, Vol. 18, No. 4, April 1996, pp. 377-388.

44. L. Goodman and W. Kruskal, "Measures of associations for cross-validations", Journal of the American Statistical Association, Vol. 49, 1954, pp. 732-764.

45. Google, http://www.google.com

46. A. D. Gordon, "Hierarchical Classification", in Clustering and Classification, P. Arabie, L. J. Hubert, and G. De Soete (Eds.), World Scientific Publishing Company, Singapore, 1996, pp. 65-122.

47. S. C. Grossman, "Chemical ordering of molecules: a graphic theoretical approach to structure-property studies", International Journal of Quantum Chemistry, Vol. 28, No. 1, 1985, pp. 1-16.

48. D. A. Grossman and O. Frieder, Information Retrieval: Algorithms and Heuristics, Klewer Academic Publishers, Norwell, MA,1998.

49. Grouper II, http://www.cs.washington.edu/research/clustering

50. S. Günter and H. Bunke, "Self-organizing map for clustering in the graph domain", Pattern Recognition Letters, Vol. 23, 2002, pp. 405-417.

51. P. Hannappel, R. Klapsing, and G. Neumann, "MSEEC - A Multi Search Engine with Multiple Clustering", Proceedings of the 99 Information Resources Management Association International Conference, May 1999.

52. A. Hardy, "On the number of clusters", Computational Statistics \& Data Analysis, Vol. 23, 1996, pp. 83-96.

53. K. Haris, S. N. Efstratiadis, N. Maglaveras, C. Pappas, J. Gourassas, and G. Louridas, "Model-Based Morphological Segmentation and Labeling of Coronary Angiograms", IEEE Transactions on Medical Imaging, Vol. 18, No. 10, October 1999, pp. 1003-1015.

54. X. He, C. Ding, H. Zha, and H. D. Simon, "Automatic Topic Identification Using Webpage Clustering", Proceedings of the IEEE International Conference on Data Mining, 2001, pp. 195-202, 2001.

55. M. Hearst and J. Pedersen, "Reexamining the Cluster Hypothesis: Scatter/Gather on Retrieval Results", Proceedings of the $19^{\text {th }}$ Annual International ACM/SIGIR Conference on Research and Development in Information Retrieval, 1996.

56. L. Hubert and J. Schultz, "Quadratic assignment as a general data-analysis strategy", British Journal of Mathematical and Statistical Psychology, Vol. 29, 1976, pp. 190-240.

57. B. Huet and E. R. Hancock, "Shape recognition from large image libraries by inexact graph matching", Pattern Recognition Letters, Vol. 20, 1999, pp. 1259-1269.

58. A. K. Jain and R. C. Dubes, Algorithms for Clustering Data, Prentice-Hall, Englewood Cliffs, NJ, 1988. 
59. A. K. Jain, M. N. Murty and P. J. Flynn, "Data Clustering: A Review", $A C M$ Computing Surveys, Vol. 31, No. 3, Sept. 1999, pp. 264-323.

60. K. S. Jones and M. Kay, Linguistics and Information Science, Academic Press, New York, NY, 1973.

61. G. J. Klir and B. Yuan, Fuzzy Sets and Fuzzy Logic: Theory and Applications, Prentice Hall, Upper Saddle River, 1995.

62. T. Kohonen, S. Kaski, K. Lagus, J. Salojärvi, J. Honkela, V. Paatero and A. Saarela, "Self Organization of a Massive Document Collection", IEEE Transactions on Neural Networks, Vol. 11, No. 3, May 2000, pp. 574-585.

63. R. Kosala and H. Blockeel, "Web Mining Research: A Survey", SIGKDD Explorations, Vol. 2, 2000, pp. 1-15.

64. R. Kothari and D. Pitts, "On finding the number of clusters", Pattern Recognition Letters, Vol. 20, 1999, pp. 405-416.

65. W. J. Krzanowski and F. H. C. Marriott, Multivariate Analysis Part 2: Classification, covariance structures and repeated measurements, Arnold, 1995.

66. R. Kumar, P. Raghavan, S. Rajagopalan, and A. Tomkins, "Extracting LargeScale Knowledge Bases from the Web", Proceedings of the $25^{\text {th }}$ International Conference on Very Large Databases, 1999, pp. 639-649.

67. M. Lazarescu, H. Bunke, and S. Venkatesh, "Graph matching: fast candidate elimination using machine learning techniques", Advances in Pattern Recognition, Joint IAPR International Workshops SSPR and SPR 2000, Lecture Notes in Computer Science 1876, 2000 pp. 236-245.

68. V. Levenshtein, "Binary Codes Capable of Correcting Deletions, Insertions, and Reversals", Soviet Physics-Doklady, Vol. 10, 1966, pp. 707-710.

69. G. Levi, "A note on the derivation of maximal common subgraphs of two directed or undirected graphs", Calcolo, Vol. 9, 1972, pp. 341-354.

70. J. Liang, D. Doermann, "Logical labeling of document images using layout graph matching with adaptive learning", Document Analysis Systems V, D. Lopresti, J. Hu, R. Kashi (Eds.), Springer-Verlag, Lecture Notes in Computer Science 2423, 2002, pp. 224-235.

71. A. Likas, N. Vlassis, and J. J. Verbeek, "The global k-means algorithm", Pattern Recognition, Vol. 36, 2003, pp. 451-461.

72. D. Lopresti and G. Wilfong, "Applications of graph probing to web document analysis", Proceedings of the $1^{\text {st }}$ International Workshop on Web Document Analysis (WDA2001), 2001, pp. 51-54.

73. J. B. Lovins, "Development of a Stemming Algorithm", Mechanical Translation and Computational Linguistics, Vol. 11, No. 1-2, March 1968, pp. 22-31.

74. X. Lu, "Document Retrieval: A Structural Approach", Information Processing and Management, Vol. 26, No. 2, pp. 209-218, 1990. 
7 5. B. Luo, A. Robles-Kelly, A. Torsello, R. C. Wilson and E. R. Hancock, "Clustering shock trees", $3^{\text {rd }}$ IAPR-TC15 Workshop on Graph-based Representations in Pattern Recognition (2001), pp. 217-228.

76. G. F. Luger and W. A. Stubblefield, Artificial Intelligence: Structures and Strategies for Complex Problem Solving, The Benjamin/Cummings Publishing Company, Redwood City, CA, 1993.

7 7. S. A. Macskassy, A. Banerjee, B. D. Davison, and H. Hirsh, "Human Performance on Clustering Web Pages: A Preliminary Study", Proceedings of The $4^{\text {th }}$ International Conference on Knowledge Discovery and Data Mining, 1998, pp. 264-268.

78. S. K. Madria, S. S. Bhowmick, W.-K. Ng and E.-P. Lim, "Research Issues in Web Data Mining”, Data Warehousing and Knowledge Discovery, 1999, pp. 303-312.

79. C. Magnusson and H. Vanharanta, "Visualizing Sequences of text Using Collocational Networks", Lecture Notes in Artificial Intelligence, Vol. 2734, 2003, pp. 276-283.

80. M. Marchiori, "The Quest for Correct Information on the Web: Hyper Search Engines”, Computer Networks and ISDN Systems, Vol. 29, 1997, pp. 1225-1235.

81. F. Masseglia, P. Poncelet, and R. Cicchetti, "WebTool: An Integrated Framework for Data Mining", Proceedings of the $10^{\text {th }}$ International Conference and Workshop on Database and Expert Systems Applications, 1999, pp. 892-901.

82. A. McCallum and K. Nigam, "A comparison of event models for Naive Bayes text classification”, AAAI-98 Workshop on Learning for Text Categorization, 1998.

83. J. J. McGregor, "Backtrack search algorithms and the maximal common subgraph problem", Software Practice and Experience, Vol. 12, 1982, pp. 23-34.

84. C. T. Meadow, B. R. Boyce and D. H. Kraft, Text Information Retrieval Systems, Academic Press, San Diego, CA, 2000.

85. S. Medasani, R. Krishnapuram, and Y. S. Choi, "Graph Matching by Relaxation of Fuzzy Assignments", IEEE Transactions on Fuzzy Systems, Vol. 9, No. 1, February 2001, pp. 173-182.

86. B. T. Messmer and H. Bunke, "A new algorithm for error-tolerant subgraph isomorphism detection", IEEE Transactions on Pattern Analysis and Machine Intelligence, Vol. 20, No. 5, 1998, pp. 493-504.

87. M. Merzbacher, "Discovering Semantic Proximity for Web Pages", Proceedings of the $11^{\text {th }}$ International Symposium on Methodologies for Intelligent Systems, 1999, pp. 244-252.

88. B. T. Messmer and H. Bunke, "A New Algorithm for Error-Tolerant Subgraph Isomorphism Detection", IEEE Transactions on Pattern Analysis and Machine Intelligence, Vol. 20, No. 5, May 1998, pp. 493-504.

89. G. A. Miller, "WordNet: A Lexical Database for English", Communications of the ACM, Vol. 38, No. 11, 1995, pp. 39-41. 
90. B. Mirkin, Mathematical Classification and Clustering, Kluwer Academic Publishers Group, Norwell, MA, 1996.

91. T. M. Mitchell, Machine Learning, McGraw-Hill, Boston, 1997.

92. R. Myers, R. C. Wilson, and E. R. Hancock, "Bayesian Graph Edit Distance", IEEE Transactions on Pattern Analysis and Machine Intelligence, Vol. 22, No. 6, June 2000, pp. 628-635.

93. U. Y. Nahm and R. J. Mooney, "A Mutually Beneficial Integration of Data Mining and Information Extraction", Proceedings of the $17^{\text {th }}$ National Conference on Artificial Intelligence, July 2000.

94. E. Nakamura and N. Kehtarnavaz, "Determining number of clusters and prototype locations via multi-scale clustering", Pattern Recognition Letters, Vol. 19, 1998, pp. $1265-1283$.

95. O. Nasraoui, H. Frigui, A. Joshi, and R. Krishnapuram, "Mining Web Access Logs Using Relational Competitive Fuzzy Clustering", Proceedings of the $8^{\text {th }}$ International Fuzzy Systems Association World Congress, 1999, pp. 869-873.

96. O. Owolabi, "An efficient graph approach to matching chemical structures", Journal of Chemical Information and Computer Sciences, Vol. 28, No. 4, 1988, pp. 221-226.

97. A. T. P. and C. Siva Ram Murthy, "Optimal task allocation in distributed systems by graph matching and state space search", The Journal of Systems and Software, Vol. 46, 1999, pp. 59-75.

98. N. R. Pal and J. Biswas, "Cluster validation using graph theoretic concepts", Pattern Recognition, Vol. 30, No. 6, 1997, pp. 847-857.

99. S. K. Pal, V. Talwar, and P. Mitra, "Web Mining in Soft Computing Framework: Relevance, State of the Art and Future Directions", IEEE Transactions on Neural Networks, Vol. 13, No. 5, 2000, pp. 1163-1177.

100. T. Peterson, "Coping with Infoglut", Computerworld (online article), http://www. computerworld.com/databasetopics/data/story/0,10801,82314,00.html

101. B. Piwowarski and P. Gallinari, "A Machine Learning Model for Information Retrieval with Structured Documents", Lecture Notes in Artificial Intelligence, Vol. 2734, 2003, pp. 425-438.

102. M. F. Porter, “An Algorithm for Suffix Stripping”, Program, Vol. 14, No. 3, July 1980, pp. 130-137.

103. W. M. Rand, "Objective criteria for the evaluation of clustering methods", Journal of the American Statistical Association, Vol. 66, 1971, pp. 846-850.

104. M. R. Rezaee, B. P. F. Lelieveldt, J. H. C. Reiber, "A new cluster validity index for the fuzzy c-mean”, Pattern Recognition Letters, Vol. 19, 1998, pp. 237-246. 
105. S. Rosenberg, I. Van Mechelen, P. De Boeck, "A Hierarchical Classes Model: Theory and Method with Applications in Psychology and Psychopathology", in Clustering and Classification, P. Arabie, L. J. Hubert, and G. De Soete, (Eds.), World Scientific Publishing Company, Singapore, 1996, pp. 123-155.

106. S. Russell and P. Norvig, Artificial Intelligence: A Modern Approach, PrenticeHall, Upper Saddle River, NJ, 1995.

107. G. Salton, Automatic Text Processing: The Transformation, Analysis, and Retrieval of Information by Computer, Addison-Wesley, Reading, MA, 1989.

108. A. Sanfeliu and K. S. Fu, "A distance measure between attributed relational graphs for pattern recognition", IEEE Transactions on Systems, Man, and Cybernetics, Vol. 13, 1983, pp. 353-363.

109. A. Sanfeliu, F. Serratosa, and R. Alquézar, "Clustering of attributed graphs and unsupervised synthesis of function-described graphs", Proceedings of the 15th International Conference on Pattern Recognition (ICPR'2000), Vol. 2, 2000, pp. 1026-1029.

110. A. Schenker, M. Last, and A. Kandel, "Design and Implementation of a Web Mining System for Organizing Search Engine Results", Proceedings of CAiSE'01 Workshop Data Integration over the Web (DIWeb), Interlaken, Switzerland, 4-5 June, 2001, pp. 62-75.

111. A. Schenker, M. Last, and A. Kandel, "A Term-Based Algorithm for Hierarchical Clustering of Web Documents", Proceedings of IFSA / NAFIPS 2001, Vancouver, Canada, July 25-28, 2001, pp. 3076-3081.

112. A. Schenker, M. Last, H. Bunke and A. Kandel, "Clustering of Web Documents Using a Graph Model", Web Document Analysis: Challenges and Opportunities, A. Antonacopoulos, and J. Hu (Eds.), to appear.

113. A. Schenker, M. Last, H. Bunke and A. Kandel, "Graph Representations for Web Document Clustering", Proceedings of the $1^{\text {st }}$ Iberian Conference on Pattern Recognition and Image Analysis (IbPRIA), to appear.

114. A. Schenker, M. Last, H. Bunke and A. Kandel, "Classification of Web Documents Using a Graph Model", Proceedings of the $7^{\text {th }}$ International Conference on Document Analysis and Recognition (ICDAR2003), to appear.

115. A. Schenker, M. Last, H. Bunke and A. Kandel, "Comparison of Distance Measures for Graph-based Clustering of Documents", Proceedings of the 4th IAPR-TC15 Workshop on Graph-based Representations (GbR'03), to appear.

116. A. Schenker, M. Last, H. Bunke and A. Kandel, "Classification of Documents using Graph Matching", International Journal of Pattern Recognition and Artificial Intelligence, to appear.

117. A. Schenker, M. Last, H. Bunke and A. Kandel, "A Comparison of Two Novel Algorithms for Clustering Web Documents", $2^{\text {nd }}$ International Conference on Web Document Analysis, to appear. 
118. H. Schütze and C. Silverstein, "Projections for Efficient Document Clustering", Proceedings of the $20^{\text {th }}$ Annual International ACM SIGIR Conference on Research and Development in Information Retrieval, 1997, pp. 74-81.

119. J. Stefanowski and D. Weiss, "Carrot ${ }^{2}$ and Language Properties in Web Search Results", Lecture Notes in Artificial Intelligence, Vol. 2663, p. 240-249.

120. A. Strehl, J. Ghosh, and R. Mooney, "Impact of similarity measures on web-page clustering", AAAI-2000: Workshop of Artificial Intelligence for Web Search, 2000, pp. 58-64.

121. K.-C. Tai, "The tree-to-tree correction problem", Journal of the Association for Computing Machinery, Vol. 26, No. 3, 1979, pp. 422-433.

122. C.-M. Tan, Y.-F. Wang, and C.-D. Lee, "The use of bigrams to enhance text categorization", Information Processing and Management, Vol. 38, 2002, pp. 529-546.

123. S. Theodoridis and K. Koutroumbas, Pattern Recognition, Academic Press, San Diego, CA, 1999.

124. J. R. Ullman, "An algorithm for subgraph isomorphism", Journal of the Association for Computing Machinery, Vol. 23, 1976, pp. 31-42.

125. Vivísimo, http://vivisimo.com/

126. R. A. Wagner and M. J. Fischer, "The String-to-String Correction Problem", Journal of the Association for Computing Machinery, Vol. 21, 1974, pp. 168-173.

127. W. D. Wallis, P. Shoubridge, M. Kraetz, and D. Ray, "Graph distances using graph union”, Pattern Recognition Letters, Vol. 22, 2001, pp. 701-704.

128. J. T. L. Wang, K. Zhang, and G.-W. Chirn, "Algorithms for Approximate Graph Matching”, Information Sciences, Vol. 82, 1995, pp. 45-74.

129. S. Wei, S. Jun, and Z. Huicheng, "A fingerprint recognition system by use of graph matching”, Proceedings of SPIE, Vol. 4554, 2001, pp. 141-146.

130. S. M. Weiss, C. Apte, F. J. Damerau, D. E. Johnson, F. J. Oles, T. Goetz, and T. Hampp, "Maximizing Text-Mining Performance", IEEE Intelligent Systems, Vol. 14, No. 4, Jul./Aug. 1999, pp. 63-69.

131. M. L. Williams, R. C. Wilson, and E. R. Hancock, "Multiple graph matching with Bayesian inference", Pattern Recognition Letters, Vol. 18, 1997, pp. 1275-1281.

132. L. Wiskott, J.-M. Fellous, N. Krüger and C. von der Malsburg, "Face Recognition by Elastic Bunch Graph Matching", Proceedings of the $7^{\text {th }}$ International Conference on Computer Analysis of Images and Patterns, 1997, pp. 456-463.

133. R. C. Wilson and E. R. Hancock, "Structural Matching by Discrete Relaxation", IEEE Transactions on Pattern Analysis and Machine Intelligence, Vol. 19, No. 6, June 1997, pp. 634-647.

134. R. C. Wilson and E. R. Hancock, "Graph matching with hierarchical discrete relaxation", Pattern Recognition Letters, Vol. 20, 1999, pp. 1041-1052. 
135. C.-C. Wong, C.-C. Chen, and M.-C. Su, "A novel algorithm for data clustering", Pattern Recogniton, Vol. 34, 2001, pp. 425-442.

136. N. Zahid, O. Abouelala, M. Limouri, and A. Essaid, "Unsupervised fuzzy clustering”, Pattern Recognition Letters, Vol. 20, 1999, pp. 123-129.

137. N. Zahid, M. Limouri, and A. Essaid, "A new cluster-validity for fuzzy clustering", Pattern Recognition, Vol. 332, 1999, pp. 1089-1097.

138. C. T. Zahn, "Graph-theoretical methods for detecting and describing gestalt structures", IEEE Transactions on Computers C-20, 1971, pp. 68-86.

139. O.Zamir and O. Etzioni, "Web Document Clustering: A Feasibility Demonstration", Proceedings of the $21^{\text {st }}$ Annual International ACM SIGIR Conference on Research and Development in Information Retrieval, 1998, pp. 46-54.

140. N. Zhong, J. Liu, and Y. Yao, "In Search of the Wisdom Web", Computer, Vol. 35, No. 11, 2002, pp. 27-31. 
Appendices 


\section{Appendix A: Examples of Documents Used in Experiments}

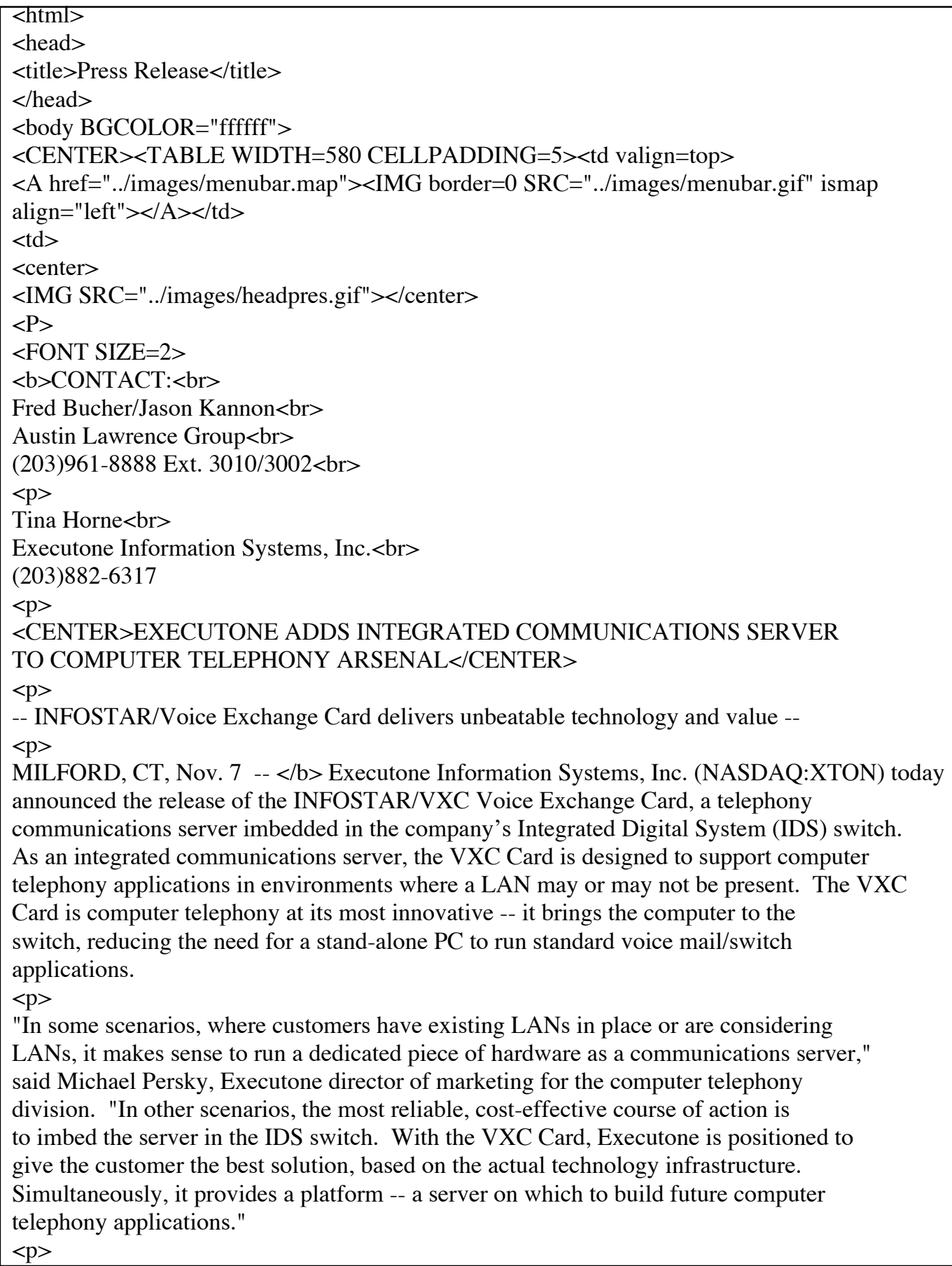

Figure A.1. Original HTML of Document \#68 of the J-Series Data Set (Partial) 


\section{Appendix A (Continued)}

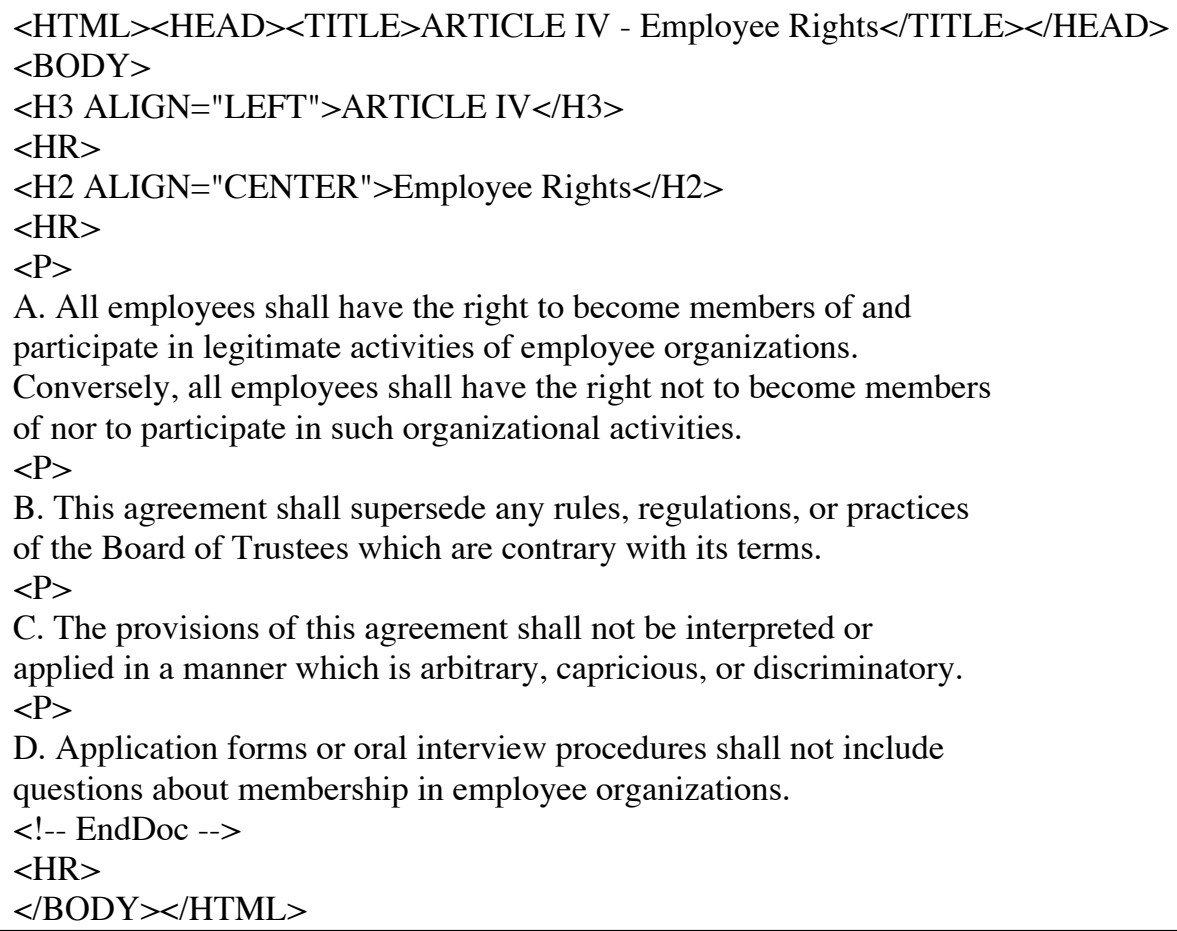

Figure A.2. Original HTML of Document \#104 of the J-Series Data Set 


\section{Appendix A (Continued)}

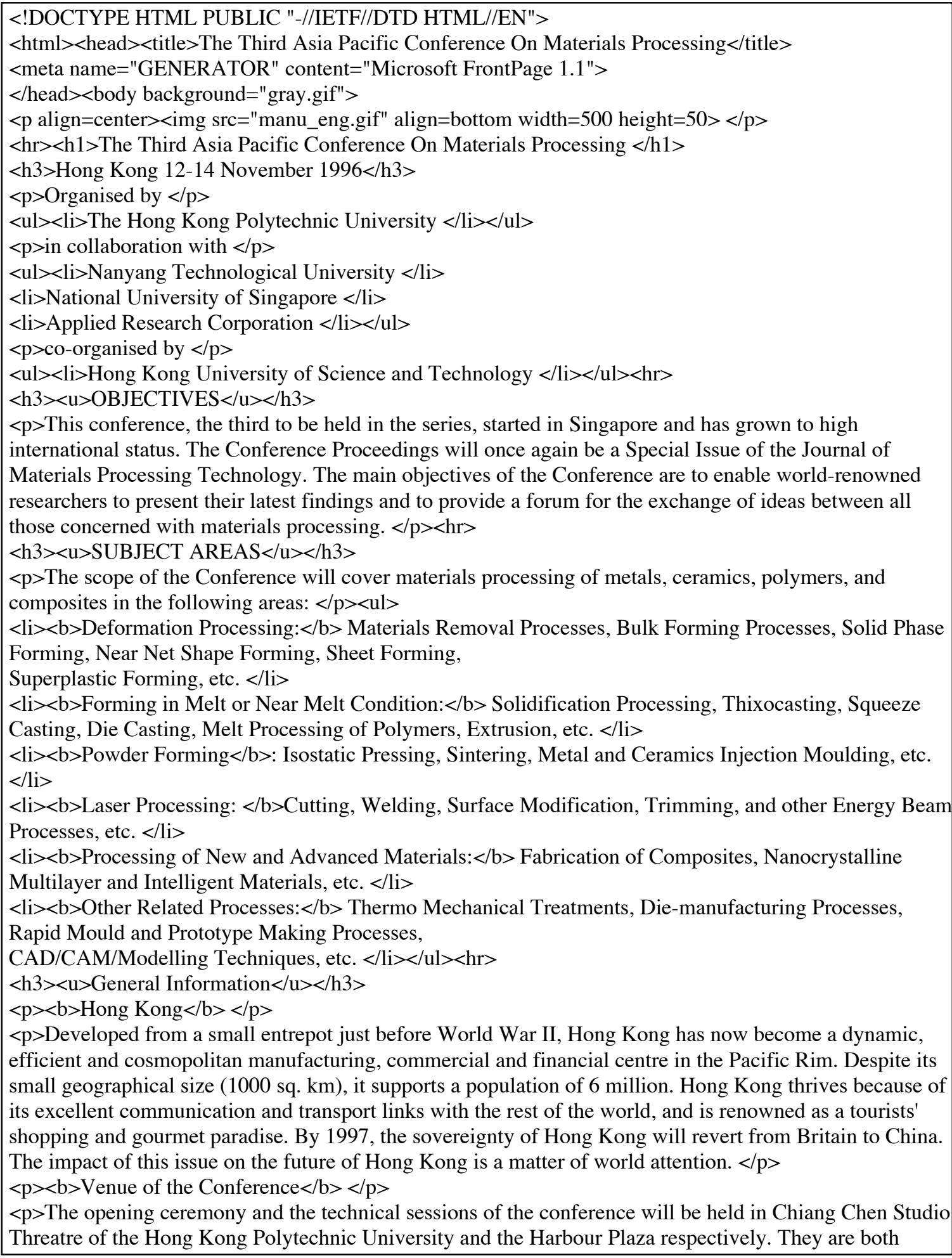

Figure A.3. Original HTML of Document \#183 of the J-Series Data Set (Partial) 


\title{
Appendix A (Continued)
}

\author{
CONTACT: \\ Fred Bucher/Jason Kannon \\ Austin Lawrence Group \\ (203)961-8888 Ext. 3010/3002 \\ Tina Horne \\ Executone Information Systems, Inc.
}

(203) $882-6317$

EXECUTONE ADDS INTEGRATED COMMUNICATIONS SERVER TO COMPUTER TELEPHONY ARSENAL.

-- INFOSTAR/Voice Exchange Card delivers unheatable technology and value -.

MILFORD, CT, Nov. 7 -- Executone Information Systems, Inc. (NASDAQ:XTON) today announced the release of the INFOSTAR/VXC Voice Exchange Card, a telephony communications server imbedded in the company's Integrated Digital System (IDS) switch. As an integrated communications server, the VXC Card is designed to support computer telephony applications in environments where a LAN may or may not be present. The VXC Card is computer telephony at its most innovative -- it brings the computer to the switch, reducing the need for a stand-alone PC to run standard voice mail/switch applications.

"In some scenarios, where customers have existing LANs in place or are considering LANs, it makes sense to run a dedicated piece of hardware as a communications server," said Michael Persky, Executone director of marketing for the computer telephony division. "In other scenarios, the most reliable, cost-effective course of action is to imbed the server in the IDS switch. With the VXC Card, Executone is positioned to give the customer the best solution, based on the actual technology infrastructure. Simultaneously, it provides a platform -- a server on which to build future computer telephony applications."

Using Digital Signal Processing (DSP) technology, the VXC Card is more reliable and cost-effective over its operating life than traditional stand-alone voice mail systems, based on the fact that there are fewer points of potential failure. Empowered by an industry-standard operating system, the VXC Card plugs directly into one of the slots in the IDS cabinet to work as an applications server along side the system common control, station and network cards.

The first available application, voice mail, is currently shipping with the VXC Card. With a full complement of voice mail features and options, including bi-lingual voice prompts, expanded outdialing, emergency notification, list outdialing and wake-up schedules, it is a powerful offering. The VXC voice mail is avalable in four, six and eight-port configurations with 45 hours of storage. Future applications on an enhanced server card will follow. The VXC Card is available, including voice mail, at retail prices ranging from $\$ 4,000$ to $\$ 10,000$.

Executone Information Systems, Inc. develops, markets and supports voice and data communications systems, and healtheare communications systems. Products and services include telephone systems, voice mail systems, inbound and outbound call center systems, specialized bealtheare communications systems and application consulting services. The company, through its Unistar Entertainment subsidiary, also has the exclusive right to design, develop and manage the National Indian Lottery. Products and services are sold under the EXECUTONE, INFOSTAR, IDS, LIFESAVER, INFOSTAR/ILS and UNISTAR brand names.

Editor's Note: For more information, visit Executone on the World Wide Web at http://www.executone.com or call 1-800-955-9866.

Figure A.4. Document of Fig. A.1 as Rendered in a Web Browser (Partial) 


\section{Appendix A (Continued)}

\section{ARTICLE IV}

\section{Employee Rights}

A. All employees shall have the right to become members of and participate in legitimate activities of employee organizations. Conversely, all employees shall have the right not to become members of nor to participate in such organizational activities.

B. This agreement shall supersede any rules, regulations, or practices of the Board of Trustees which are contrary with its terms.

C. The provisions of this agreement shall not be interpreted or applied in a manner which is arbitrary, capricious, or discriminatory.

D. Application forms or oral interview procedures shall not include questions about membership in employee organizations.

Figure A.5. Document of Fig. A.2 as Rendered in a Web Browser 


\title{
Appendix A (Continued)
}

\section{The Third Asia Pacific Conference On Materials Processing}

\author{
Hong Kong 12-14 November 1996 \\ Organised by \\ - The Hong Kong Polytechnic University \\ in collaboration with
}

- Nanyang Technological University

- National University of Singapore

- Applied Research Corporation

co-organised by

- Hong Kong University of Science and Technology

\section{OBJECTIVES}

This conference, the third to be held in the series, started in Singapore and has grown to high international status. The Conference Proceedings will once again be a Special Issue of the Journal of Materials Processing Technology. The main objectives of the Conference are to enable world-renowned researchers to present their latest findings and to provide a forum for the exchange of ideas between all those concerned with materials processing.

\section{SUBJECT AREAS}

The scope of the Conference will cover materials processing of metals, ceramics, polymers, and composites in the following areas:

- Deformation Processing: Materials Removal Processes, Bulk Forming Processes, Solid Phase Forming, Near Net Shape Forming, Sheet Forming, Superplastic Forming, etc.

- Forming in Melt or Near Melt Condition: Solidification Processing. Thixocasting, Squeeze Casting, Die Casting, Melt Processing of Polymers, Extrusion, etc.

- Powder Forming: Isostatic Pressing, Sintering, Metal and Ceramics Injection Moulding, etc

- Laser Processing: Cutting, Welding, Surface Modification, Trimming, and other Energy Beam Processes, etc.

- Processing of New and Advanced Materials:

Figure A.6. Document of Fig. A.3 as Rendered in a Web Browser (Partial) 


\section{Appendix B: Graphs Created from Example Documents of Appendix A}

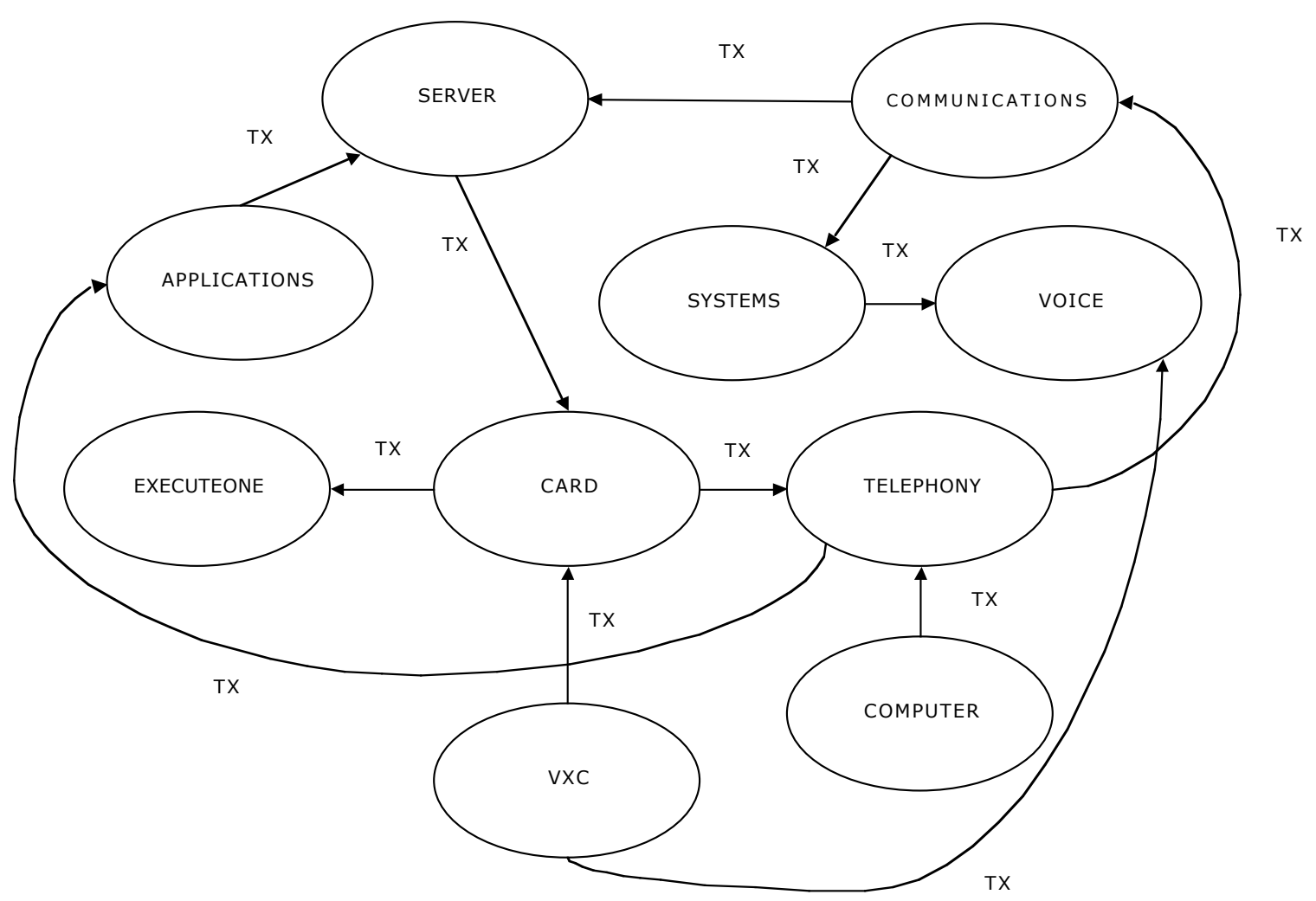

Figure B.1. Standard Graph Representation Created from Web Document of Fig. A.1 Using 10 Nodes/Graph 


\section{Appendix B (Continued)}
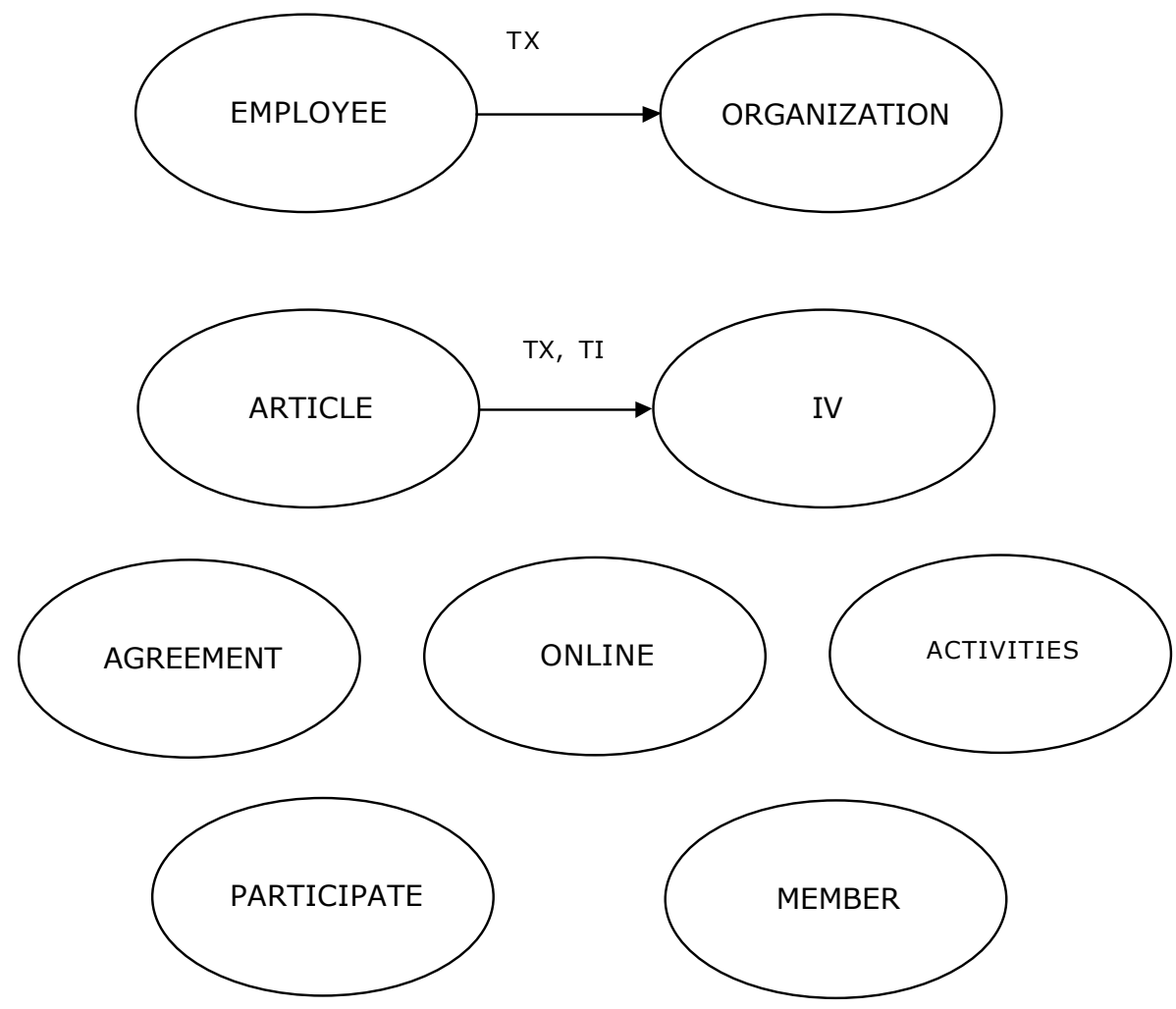

Figure B.2. Standard Graph Representation Created from Web Document of Fig. A.2 Using 10 Nodes/Graph 


\section{Appendix B (Continued)}
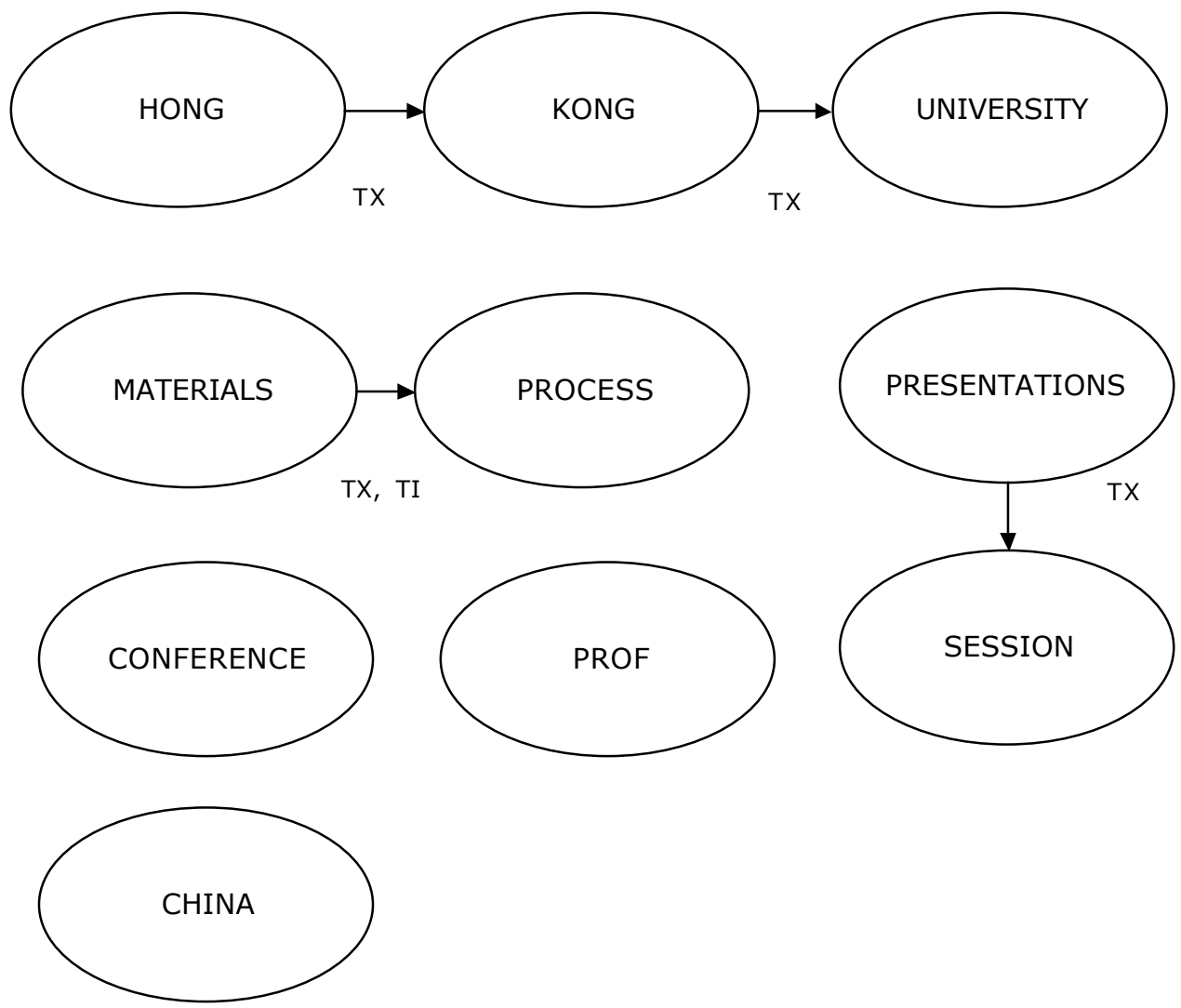

Figure B.3. Standard Graph Representation Created from Web Document of Fig. A.3 Using 10 Nodes/Graph 


\section{Appendix C: Nearest Neighbors of Example Documents}

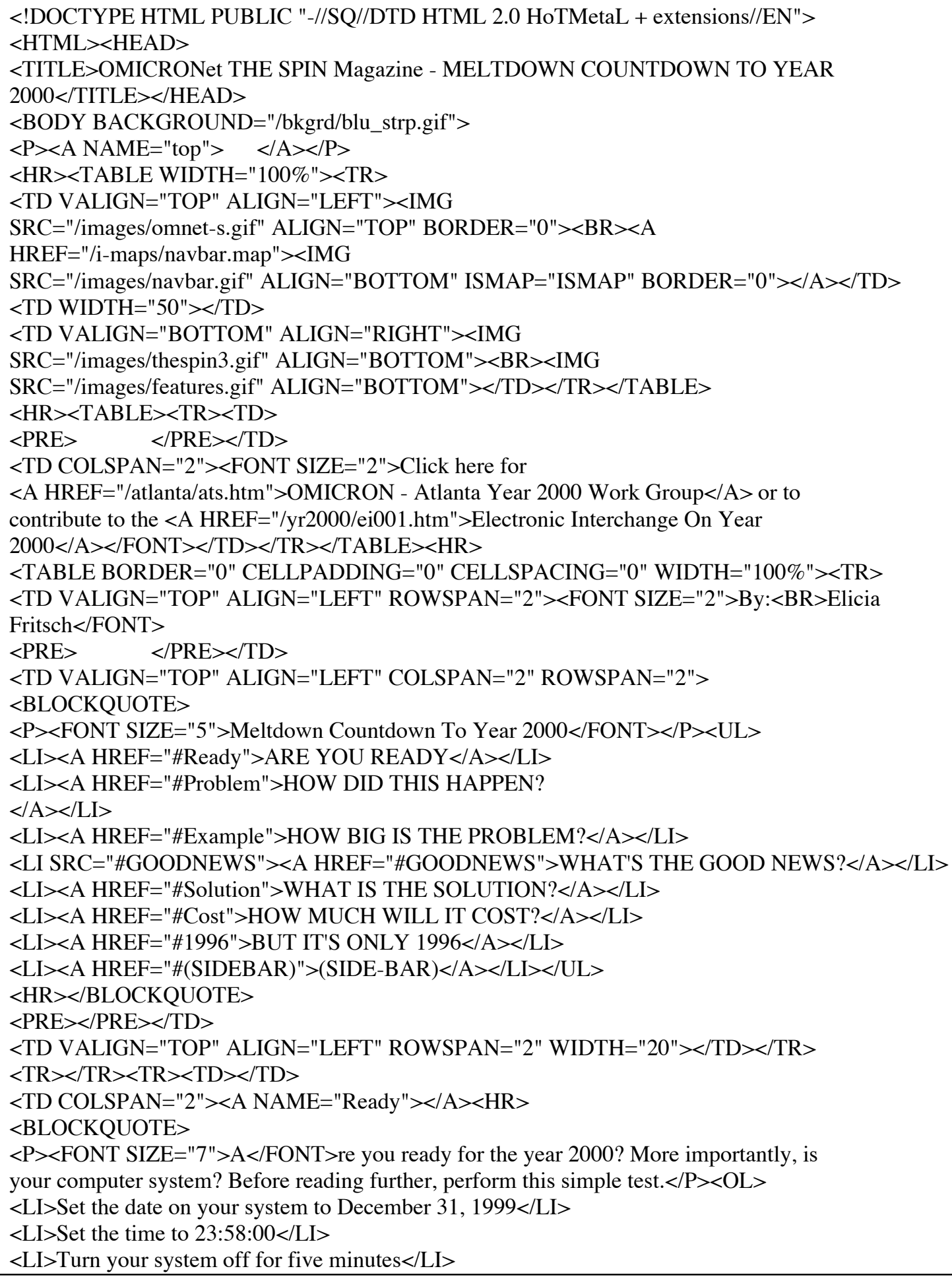

Figure C.1. HTML Source of Nearest Neighbor of Document of Fig. A.1 (Partial) 


\title{
Appendix C (Continued)
}

\author{
$<$ HTML $><$ HEAD $>$ \\ $<$ TITLE $>$ Intellectual Property Rights $</$ TITLE $><$ HEAD $>$ \\ $<$ BODY background=images/1056a.jpg $>$ \\ $<$ HR size $=10><\mathrm{P}>$ \\ $<\mathrm{H} 1>$ Intellectual Property Rights $</ \mathrm{H} 1>$ \\ $<\mathrm{HR}$ size $=10><\mathrm{P}>$
}

The WTO Agreement on Trade-Related Aspects of Intellectual Property Rights

(TRIPS) recognizes that widely varying standards in the protection and enforcement of intellectual property rights and the lack of multilateral disciplines dealing with international trade in counterfeit goods have been a growing source of tension in international economic relations. With that in mind, the agreement addresses the applicability of basic GATT principles and those of relevant international intellectual property agreements; the provision of adequate intellectual property rights; the provision of effective enforcement measures for those rights; multilateral dispute settlement; and transitional implementation arrangements. $<\mathrm{P}>$

Part I of the agreement sets out general provisions and basic principles, notably a national-treatment commitment under which nationals of other members must be given treatment no less favourable than that accorded to a member's own nationals with regard to the protection of intellectual property. It contains a most-favoured-nation clause under which any advantage a member gives to the nationals of another member must normally be extended to the nationals of all other members, even if such treatment is more favourable than that which it gives to its own nationals. $\langle\mathrm{P}\rangle$

Part II addresses different kinds of intellectual property rights. It seeks to ensure that adequate standards of intellectual property protection exist in all members countries, taking as a starting point the substantive obligations of the main pre-existing conventions of the World Intellectual Property Organization (WIPO) - namely, the Paris Convention for the Protection of Industrial Property and the Berne Convention for the Protection of Literary and Artistic Works (copyright). It adds a significant number of new or higher standards where the exuisting conventions were silent or thought inadequate $<\mathrm{P}>$

With respect to copyright, the agreement ensures that computer programs will be protected as literary works under the Berne Convention and outlines how data bases should be protected. $<\mathrm{P}>$

An important addition to existing international rules in the area of copyright and related rights is the provision on rental rights. Authors of computer programmes and producers of sound recordings have the right to authorize or prohibit the commercial rental of their works to the public. A similar exclusive right applies to films where commercial rental has led to widespread copying which is materially impairing the right of reproduction. Performers are protected from unauthorized recording, reproduction and broadcast of live performances (bootlegging) for no less than 50 years. Producers of sound recordings must have the right to prevent the reproduction of recordings for a period of 50 years. $<\mathrm{P}>$

The agreement defines what types of signs must be eligible for protection as trademarks or service marks and what the minimum rights conferred on their owners must be. Marks that have become well-known in a particular country enjoy additional protection. The agreement identifies a number of obligations for the use of trademarks and service marks, their terms of protection, and their licensing or assignment. For example, requirements that foreign marks be used in conjunction with local marks will, as a general rule, be prohibited.

$<\mathrm{P}>$

In respect of geographical indications, members must provide means to prevent the use of any indication which misleads the consumer as to the origin of goods, and any use which would constitute an act of unfair competition. Higher levels of protection are provided for geographical indications for wines and spirits, which are protected even where there is no danger of the public's being misled as to the true origin. Some exceptions are allowed, for example for names which are generic terms, but any country using such

Figure C.2. HTML Source of Nearest Neighbor of Document of Fig. A.2 (Partial) 


\section{Appendix C (Continued)}

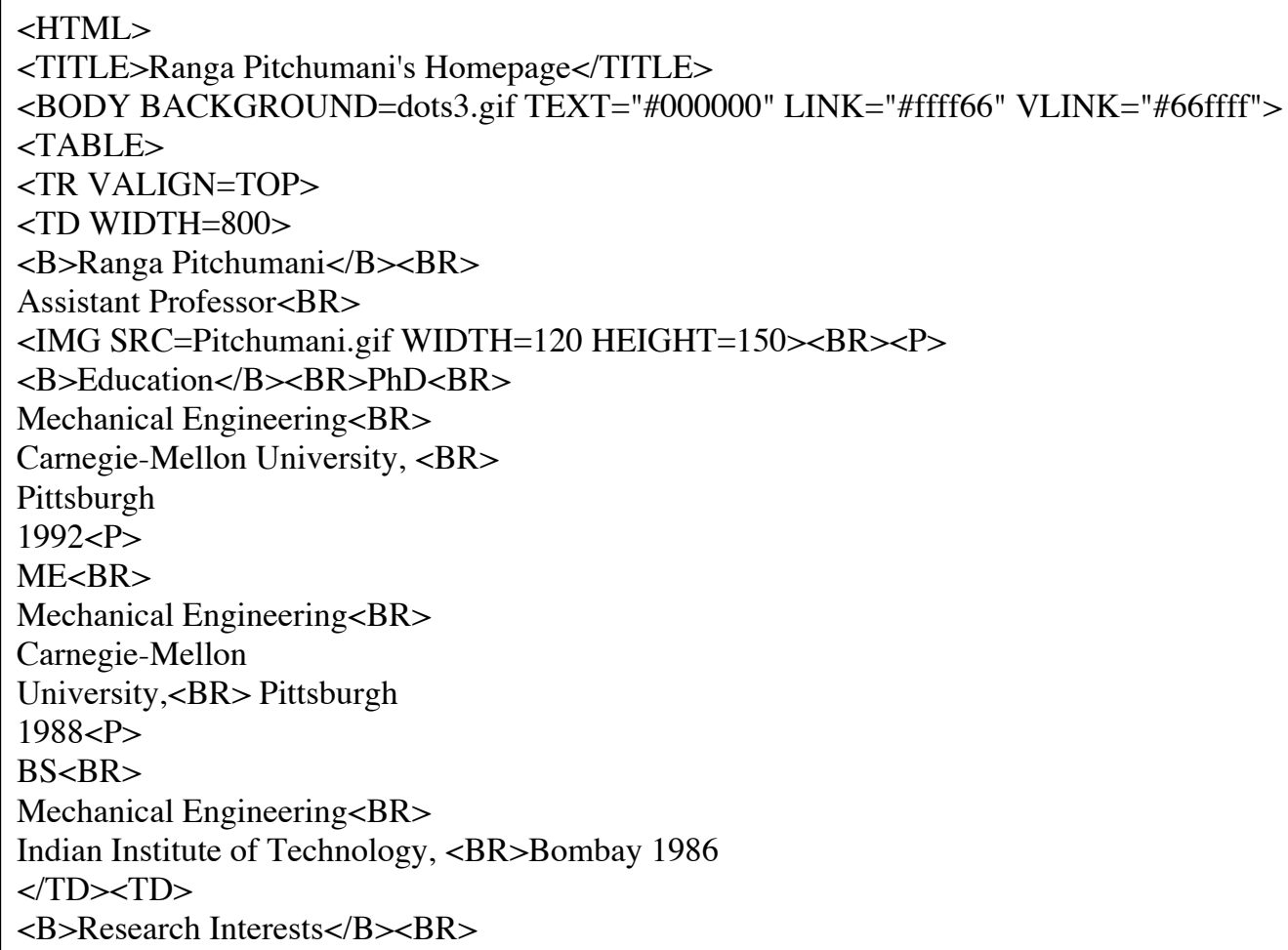

Figure C.3. HTML Source of Nearest Neighbor of Document of Fig. A.3 (Partial) 


\section{Appendix C (Continued)}

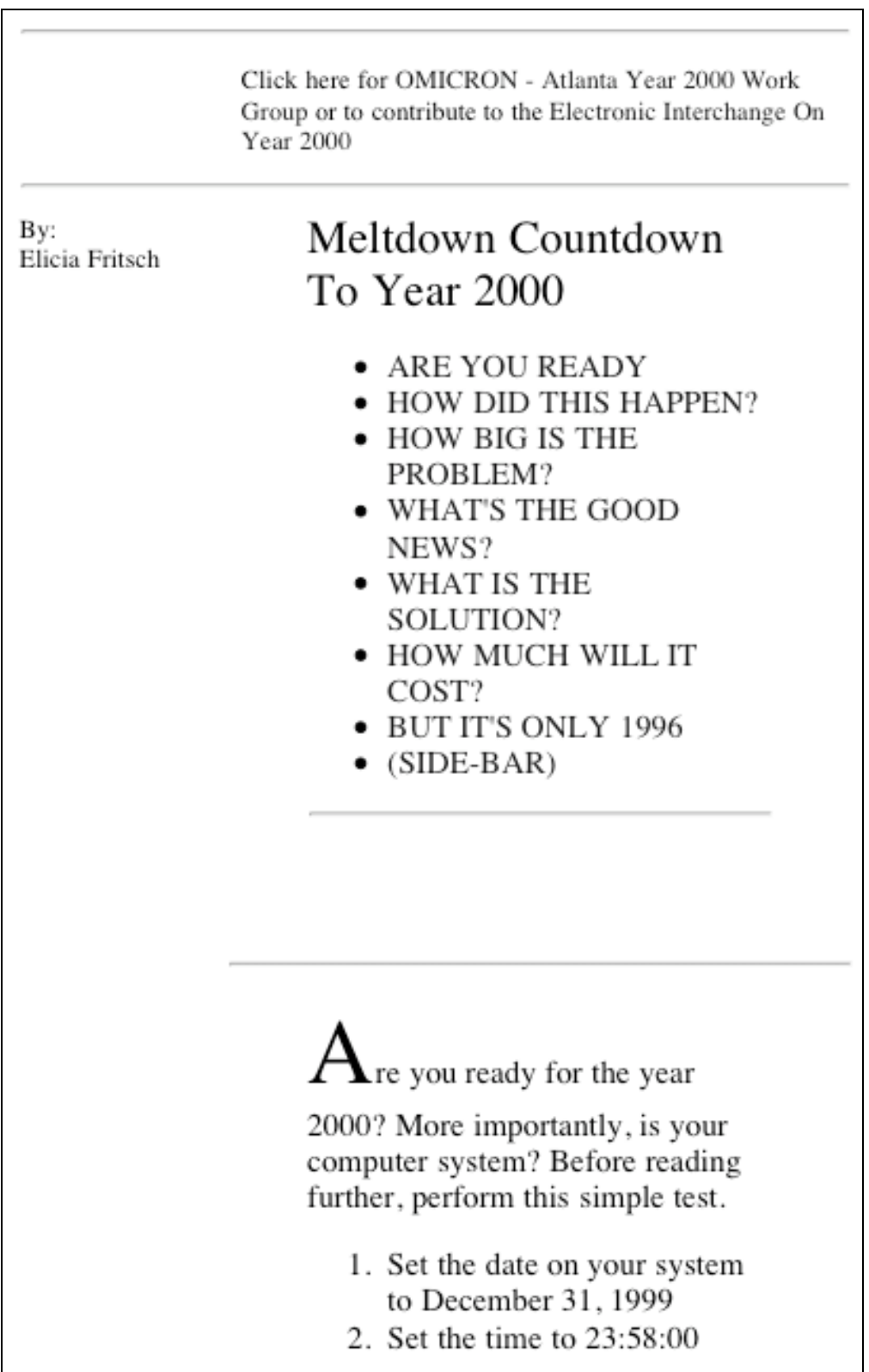

Figure C.4. Document of Fig. C.1 as Rendered in a Web Browser (Partial) 


\section{Appendix C (Continued)}

\section{Intellectual Property Rights}

The WTO Agreement on Trade-Related Aspects of Intellectual Property Rights (TRIPS) recognizes that widely varying standards in the protection and enforcement of intellectual property rights and the lack of multilateral disciplines dealing with international trade in counterfeit goods have been a growing source of tension in international economic relations. With that in mind, the agreement addresses the applicability of basic GATT principles and those of relevant international intellectual property agreements; the provision of adequate intellectual property rights; the provision of effective enforcement measures for those rights; multilateral dispute settlement; and transitional implementation arrangements.

Part I of the agreement sets out general provisions and basic principles, notably a national-treatment commitment under which nationals of other members must be given treatment no less favourable than that accorded to a member's own nationals with regard to the protection of intellectual property. It contains a most-favoured-nation clause under which any advantage a member gives to the nationals of another member must normally be extended to the nationals of all other members, even if such treatment is more favourable than that which it gives to its own nationals.

Part II addresses different kinds of intellectual property rights. It seeks to ensure that adequate standards of intellectual property protection exist in all members countries, taking as a starting point the substantive obligations of the main pre-existing conventions of the World Intellectual Property Organization (WIPO) - namely, the Paris Convention for the Protection of Industrial Property and the Berne Convention for the Protection of Literary and Artistic Works (copyright). It adds a significant number of new or higher standards where the

Figure C.5. Document of Fig. C.2 as Rendered in a Web Browser (Partial) 


\section{Appendix C (Continued)}

\begin{tabular}{|ll|}
\hline Ranga Pitchumani & Research Interests \\
Assistant Professor & Materials \\
& Processing/Manufacturing, \\
& transport phenomena in \\
& manufacturing, composite \\
& materials, process design \\
& and optimization, \\
& concurrent engineering, \\
& artificial intelligence \\
& applications in \\
& manufacturing \\
& Most Recent \\
Education & Publications \\
PhD & Steiner, K.V., Bauer, \\
Mechanical Engineering & B.M., Pitchumani, R., and \\
Pittsburgh 1992 & Gillespie, J.W., Jr. \\
ME & "Experimental \\
Mechanical Engineering & Verification of Modeling \\
Carnegie-Mellon University, & and Control for \\
Pittsburgh 1988 & Thermoplastic Tow \\
BS & Placement," in \\
Mechanical Engineering & Proceedings of the 40th \\
Indian Institute of Technology, & International SAMPE \\
Bombay 1986 & Symposium and \\
& Exhibition, 1995. \\
& Pitchumani, R., Liaw, \\
& P.K., Hsu, D.K., Yao, \\
& S.C., "An Eddy Current \\
& Technique for the \\
& Measurement of \\
& Constituent volume \\
& Fractions in a Three-Phase \\
& Metal-Matrix Composite," \\
& Journal of Composite \\
& Materials, 28(18), \\
& 1742-1769, 1994. \\
& \\
& \\
& \\
& \\
& \\
& \\
&
\end{tabular}

Figure C.6. Document of Fig. C.3 as Rendered in a Web Browser (Partial) 


\section{Appendix D: Graphs of Nearest Neighbors}

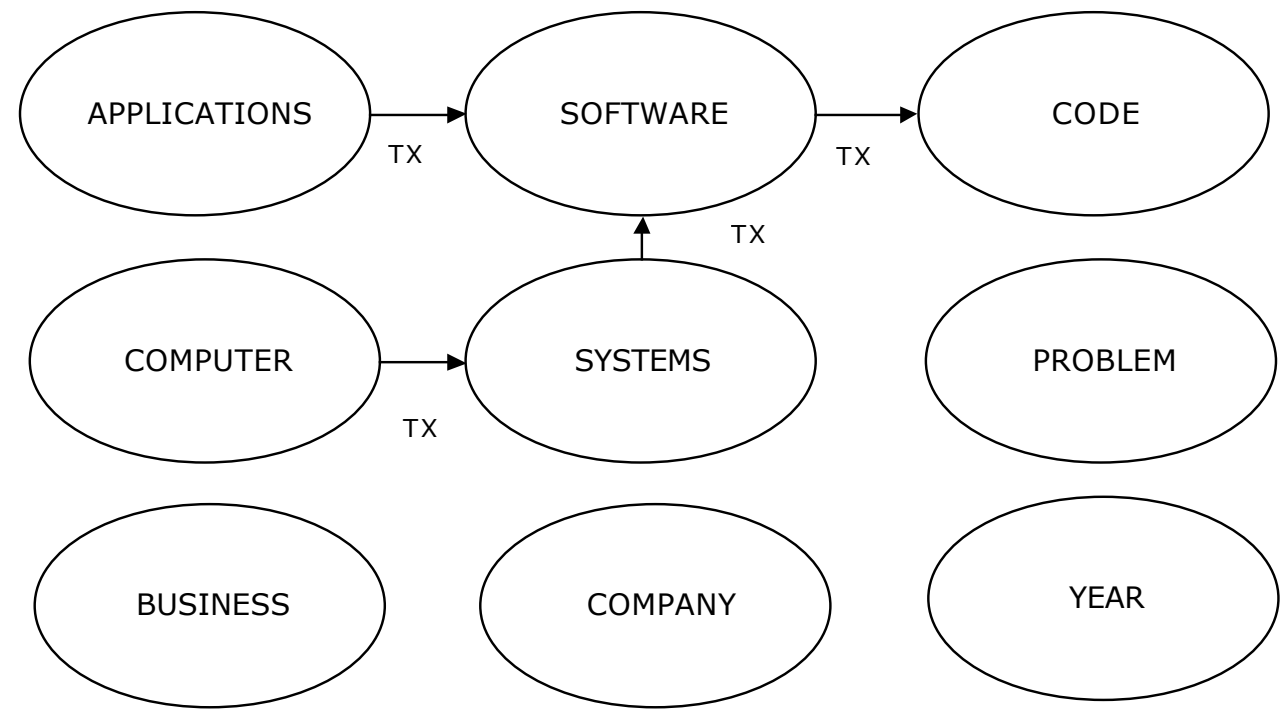

Figure D.1. Standard Graph Representation of Document of Fig. C.1 


\section{Appendix D (Continued)}

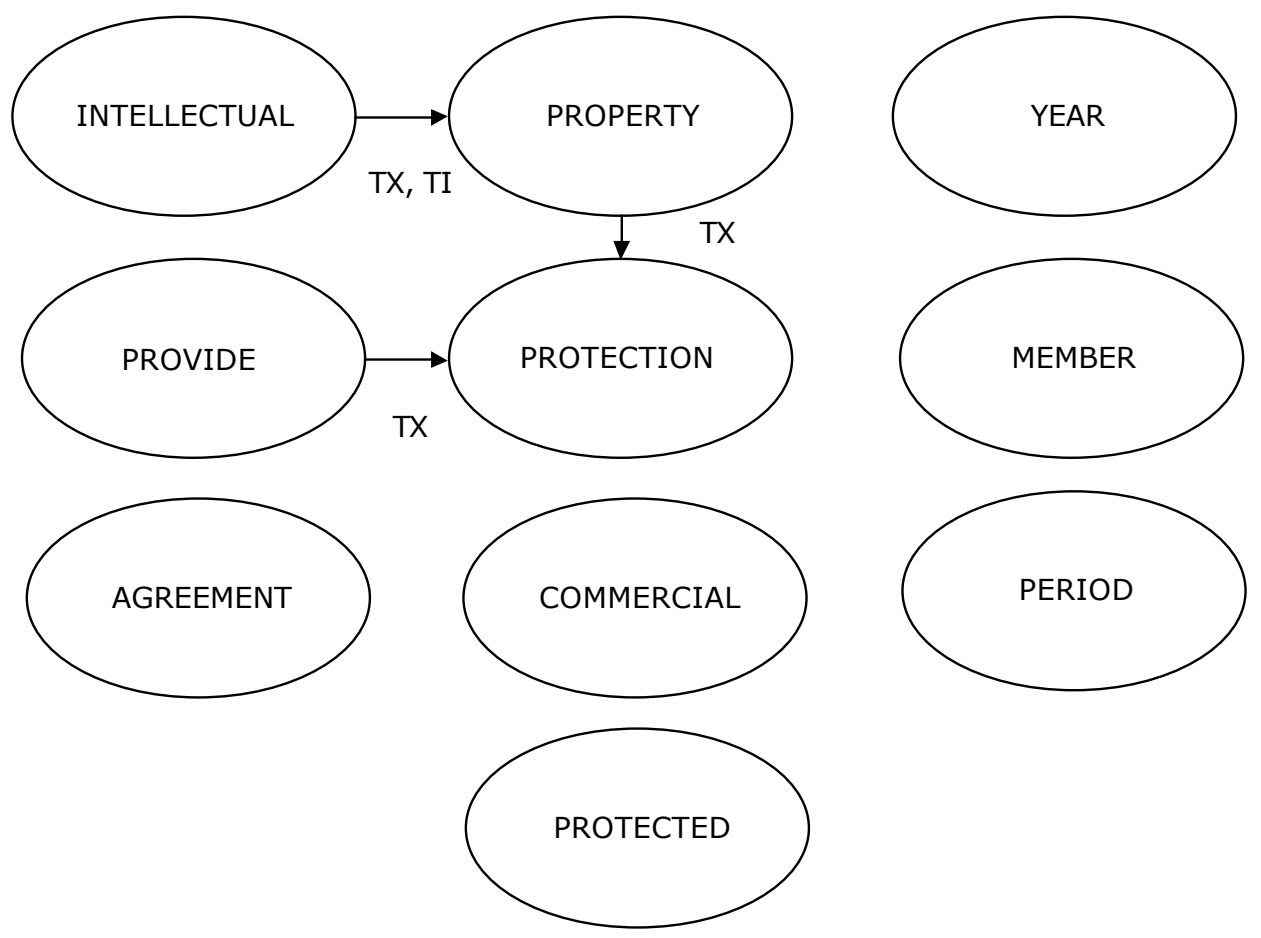

Figure D.2. Standard Graph Representation of Document of Fig. C.2 


\section{Appendix D (Continued)}

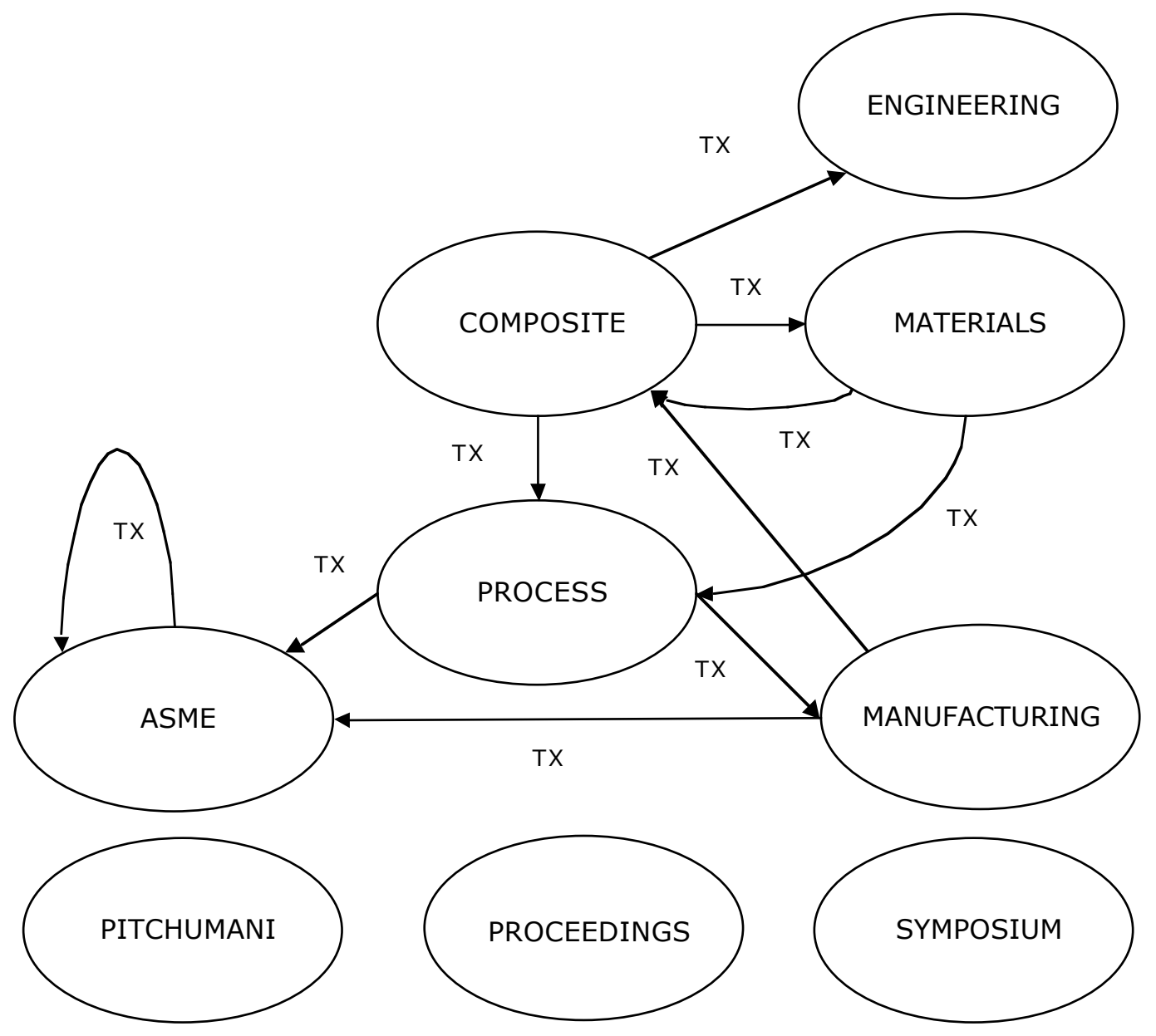

Figure D.3. Standard Graph Representation of Document of Fig. C.3 


\begin{abstract}
About the Author
Adam Schenker received the M.S. degree in Computer Engineering from the University of South Florida in 1999. He is a member of Tau Beta Pi, Upsilon Pi Epsilon, and Phi Kappa Phi. He has published several papers related to soft computing techniques and data mining which have appeared in journals such as Fuzzy Economic Review, the International Journal of Image and Graphics, the International Journal of Pattern Recognition and Artificial Intelligence, and the International Journal of Fuzzy Systems. $\mathrm{He}$ has been a Research Assistant at the National Institute of Systems Test and Productivity at the University of South Florida since 2001. His current research interests include data mining, soft computing and software testing.
\end{abstract}

